\title{
Taxonomic-faunistic notes on the jumping spiders of the Mediterranean (Aranei: Salticidae)
}

\author{
Таксономически-фаунистические заметки о пауках-скакунчиках \\ Средиземноморья (Aranei: Salticidae)
}

\author{
D.V. Logunov \\ A.B. Аогунов \\ The Manchester Museum, University of Manchester, Oxford Road, Manchester M13 9PL, UK. E-mail: dmitri.v.logunov@manchester.ac.uk
}

KEY WORDS: Spiders, Aranei, Salticidae, the Mediteranean, descriptions, new species, taxonomy, faunistic.

КЛЮЧЕВЫЕ СЛОВА: Пауки, Aranei, Salticidae, Средиземноморье, описания, новые виды, таксономия, фаунистика.

ABSTRACT. The paper presents new taxonomic and faunistic data on 162 species of the Mediterranean jumping spiders (Salticidae). Three new species are described: Heliophanus haymozi sp.n. ( ${ }^{\top}$; ; Spain, Portugal), Heliophanus sinaicus sp.n. ( $\sigma^{7}+$; Egypt, Sinai) and Salticus ressli sp.n. (O'; Turkey). Two new synonyms are proposed: Chalcoscirtus helverseni Metzner, 1999 with C. parvulus Marusik, 1991 and Salticus marenzelleri Nosek, 1905 with Salticus tricinctus (C.L. Koch, 1846). The synonymy of Evarcha syriaca Kulczyński, 1911 with Evarcha patagiata (O. PickardCambridge, 1872) is confirmed. Unknown sexes are described for seven species for the first time: Habrocestum latifasciatum (Simon, 1868) (+), H. shulovi Prószyński, $2000\left(\sigma^{7}\right)$, Heliophanus fuerteventurae Schmidt et Krause, 1996 (†), H. ramosus Wesołowska, $1986(+)$, Icius crassipes (Simon, 1868) (+), I. insolitus Alicata et Cantarella, $1994(+)$ and Salticus confusus Lucas, $1847(+)$. New faunistic records are provided for the following countries: Armenia (2 new species records), Cyprus (19), Egypt (4), Greece (1), Israel (1), Italy (1), Jordan (2), Libya (1), Morocco (4), Oman (1), Portugal (3), Spain (3), Syria (8), Tunisia (1), Turkey (17), Ukraine (1).

РЕЗЮМЕ. В статье приводятся данные по таксономии и фаунистике для 162 видов пауков-скакунчиков из Средиземноморья (Salticidae). Описано три новых вида: Heliophanus haymozi sp.n. (Оº; Испания, Португалия), Heliophanus sinaicus sp.n. (оㅇ; Египт, Синай) и Salticus ressli sp.n. (О; Турция). Предложено два новых синонима: Chalcoscirtus helverseni Metzner, 1999 c C. parvulus Marusik, 1991 и Salticus marenzelleri Nosek, 1905 с Salticus tricinctus (C.L. Koch, 1846). Подтверждена синонимия Evarcha syriaca Kulczyński, 1911 c Evarcha patagiata (O. Pickard-Cambridge, 1872). Впервые описаны неизвестные пола для семи видов: Habrocestum latifasciatum
(Simon, 1868) (+), H. shulovi Prószyński, $2000\left(0^{7}\right)$, Heliophanus fuerteventurae Schmidt et Krause, 1996 (+), H. ramosus Wesołowska, 1986 (), Icius crassipes (Simon, 1868) (+), I. insolitus Alicata et Cantarella, 1994 (†) и Salticus confusus Lucas, 1847 (†). Новые фаунистические находки даны для следующих стран: Армения (отмечены 2 новых видов), Кипр (19), Египет (4), Греция (1), Израиль (1), Италия (1), Иордан (2), Ливия (1), Марокко (4), Оман (1), Португалия (3), Испания (3), Сирия (8), Тунис (1), Турция (17), Украина (1).

\section{Introduction}

The jumping spiders (Salticidae) of the Mediterranean constitute a very rich and insufficiently studied fauna. According to Canard [2005], the salticid fauna of Europe and the Mediterranean basin consists of 508 valid species of 61 genera (plus 73 invalid names). Of them, 68 species $(13 \%)$ are known from the females only, and $75(15 \%)$ from the males. The surprising thing is that 172 salticid species $(34 \%)$ are still known from the original description and/or a single locality only. Thus, at present, neither the regional diversity, nor the distribution patterns of Mediterranean Salticidae can be considered well studied. The best studied salticid faunas of the Mediterranean are those of the Iberian Peninsula [Cardoso, Morano, 2010], Italy [Trotta, 2005; Pantini, Isaia, 2014], Greece [Metzner, 1999; Bosmans, Chatzaki, 2005], and Israel [Prószyński, 2003; Zonstein, Marusik, 2013]; for more details see below under 'Discussion'.

The aims of the present paper are as follows: (1) to provide new faunistic data for 162 species of the Mediterranean salticids in order to clarify their distribution; (2) to describe three new species; and (3) to describe unknown sexes for seven pooly known species. 


\section{Material and methods}

This work is based on extensive museum and private collections of the Mediterranean jumping spiders which were sent to the author for identification. A total of 2492 adult specimens belonging to 162 species of Salticidae has been studied. In some cases, additional salticid materials from Central Europe, the Balkans, the Caucasus, Central Asia and Iran are also included in the section 'Material'. The depositories of the studied material are abbreviates in the text as follows: BMNH - British Museum of Natural History, London, UK (J. Beccaloni); FMNH - Finnish Museum of Natural History, University of Heslinki, Finland (P. Cardoso); HUJI - The Hebrew University of Jerusalem, Zoological Department, Israel (G. Levy and E. Gavish-Regev); LMNM - Liverpool Museum, National Museums Liverpool, Liverpool, UK (G. Knight); MMUM - Manchester Museum, University of Manchester, Manchester, UK (D.V. Logunov); MVHN Museu Valencia d'Història Natural, Valencia, Spain (S.M. Alario); NHMW - Naturhistorisches Museum, Wien, Austria (J. Gruber); NHRS — Swedish Museum of Natural History, Stockholm (T. Kronestedt and G. Lindberg); MHNG - Museum d'historie naturelle, Gèneve, Switzerland (P. Schwendinger); NMPC - National Museum of Prague, Czech Republic (Å. Kurka); NUAM - Arachnology Museum of the Niğde University, Niğde, Turkey (A. Topçu); OUMNH - Oxford University Museum of Natural History, UK (Z. Simmons); PCDM - Personal collection of D. McCowan (UK); PCJK - Personal collection of Johan Van Keer (Kapelle-Op-Den-Dos, Belgium); PCJM - Personal collection of J. Murphy (Hampton, UK); PCJW Personal collection of J. Wunderlich (Hirschberg, Germany); PCLJ — Personal collection of L. Jonsson (Kristianstad, Sweden); PCMA — Personal collection of M. Askins (Swindon, Wiltshire, UK); PCMJ — Personal collection of M. Janssen (Meeuwen, Belgium); PCMS Personal collection of M. Schäfer (Berlin, Germany); PCRB - Personal collection of R. Bosmans (Gent, Belgium); PCRG - Personal collection of Richard Gallon (Llandudno, UK); PCSD - Personal collection of R.G. Snazell (Swanage, Dorset, UK), UK; PCRS Personal collection of A. Russell-Smith (Kent, UK), now partly in the MMUM; SMFM - Naturmuseum und Forschungsinstitut Senckenberg, Frankfurt am Main, Germany (P. Jäger and J. Altmann); SZMN - Siberian Zoological Museum, Institute for Systematics and Ecology of Animals, Novosibirsk, Russia (G.N. Azarkina); ZISP - Zoological Institute of the Russian Academy of Sciences, St-Petersburg, Russia (V.A. Krivokhatsky); ZMTU - Zoological Museum, University of Turku, Finland (S. Koponen and V. Vahtera); ZMUM - Zoological Museum of the Moscow University, Moscow, Russia (K.G. Mikhailov); ZTAU — Zoological Museum, Tel Aviv University, Israel (S.L. Zonstein).

The abbreviations used in the text: Eyes: AME anterior median eye, PLE - posterior lateral eye. Leg segments: Fm - femur, $\mathrm{Pt}$ - patella, Tb - tibia, Mt - metatarsus. Position of spines on legs: ap — apical, $\mathrm{d}$ - dorsal, pr - prolateral, $\mathrm{rt}$ - retrolateral, $\mathrm{v}$ ventral. For the leg spination the system adopted is that used by Ono [1988]. Other abbreviations: A.No. accession number; nr. - near. Collectors' names of those who collected more than 2-3 samples are abbreviated as follows: $\mathrm{AC}-\mathrm{A} . \mathrm{N}$. Chemeris; $\mathrm{AD}-\mathrm{A}$. Diakonov; AF - A. Fowles; AG - A.A. Gusakov; ARS - A. Russell-Smith; AS - A. Schönhofer; BH - B. Hauser; CF - C. Felton; CL - C. Lienhard; CR C.F. Roewer; DD - D. Dominguez et al.; DL - D.V. Logunov; DMC - D. McCowan; ED - E. Duffey; EG - E.F. Guseinov; EM - E.A. Mikhailova; EP E. Popov; GL - G. Lampel; HDK - H. De Koninck; HE - H. Eikamp; HEH - H. El-Hennawy; HK - H. Kahmann; JA — J. Altmann; JFM - J. \& F. Murphy; JG - J. Gruber, F. Ressl \& A. Radda; JM - J. Meier; JMS - J. Martens; KE - K. Eckl; KK - K. Kunt; KM - K.G. Mikhailov; LD — L.J. Dobroruka; LJ L. Jonsson; LM - L. Mausehund; MA - M. Askins; MB - M. Blamore; MR - M. Řezáč; PC - P. Cardoso; PD - P.M. Dunin; PH - P.J. Haymoz; PJ P. Jäger; PL - P.T. Lehtinen; PP - P. Poot; RB - R. Bosmans; RK - R. Kinzelbach; RS - R.G. Snazell; SG - S.I. Golovatch; SH - S. Huber; SJ - S. Judd; $\mathrm{SM}$ - S. Montagud et al.; SV - S.V. Vasilenko; SZ - S. Zarooni; TK — T. Kronestedt; UK - U. Kluge. The sequence of leg segments in measurement data is as follows: femur + patella + tibia + metatarsus + tarsus. All measurements are in mm. The nomenclature for distributional patterns of Salticidae follows Logunov \& Marusik [2000b]; the latter work contains a detailed account on how each range type can be specified using its both longitudinal and latitudinal components.

\section{Descriptions}

\section{Habrocestum latifasciatum (Simon, 1868)} Figs 1-3, 7-9.

Attus latifasciatus Simon, 1868: 536 (D $\sigma^{7}$; holotype $\sigma^{7}$ from Muséum National d'Histoire Naturelle, Paris, France; not examined).

For a compete set of taxonomic references see WSC [2015].

MATERIAL. TURKEY: $3 \sigma^{\top} \sigma^{\top}$ (PCRS), Akyaka, on rocks behind Cinan Beach, 30.05.1996, ARS; 5 O (PCRS), Akyaka, Sulemin's castle, under stones, 28.05.1996, ARS; 1 7 (LMNM), Içel, Tekmen, terraced cultivated slopes above sea, 8.06.1993, CF; $1 \sigma^{\prime \prime}$ (LMNM), Içel, c. $31 \mathrm{~km} \mathrm{~N}$ of Anamur $\left(36^{\circ} 12^{\prime} 11^{\prime \prime} \mathrm{N}, 32^{\circ} 54^{\prime}\right.$ $\left.01^{\prime \prime} \mathrm{E}\right)$, young, oat grazed, plantation on high, rock strewn, mountain slope, 7.05.1994, CF; $2 \sigma^{7} \sigma^{7}$ (LMNM), Içel, Narlıkuyu (36 $26^{\prime}$ $\left.04^{\prime \prime} \mathrm{N}, 34^{\circ} 06^{\prime} 44^{\prime \prime} \mathrm{E}\right)$, garrigue vegetated limestone dominated by Pinus, 5.06.1994, MB; $1 \sigma^{7}$ (SMFM), TR Kidirak S of Fethiye (Muğla), river valley and slopes $\left(36^{\circ} 31^{\prime} \mathrm{N}, 2^{\circ} 08^{\prime} \mathrm{E}\right), 24-28.05 .1988$, RK; 3 O $^{\top}, 6$ + 9 (ZMUM), Antalya, Kemer, pine forest, 50-400 m a.s.1., 23-27.04.2001, KM; 1 + (MMUM), Antalya Prov., Alanya Distr. (no exact locality), 6.11. 2003, KK; 2 oq (ZMUM), Antalya, Yarpuz, open old forest (stony chalky), 27.05.2004, LJ; 1 ( (PCRS), Akyaka, Sulemin's castle, under stones, 28.05.1996, ARS; 2 우 (PCRS), same locality, under stones in pine wood, 29.05.1996, 



Figs 1-6. Male palps of Habrocestum latifasciatum (Simon, 1868) (1-3; Turkey, Antalya, Kemer) and H. shulovi Prószyński, 2000 (4-6; Turkey, Namrun): 1, 4 - prolateral view; 2, 5 - ventral view; 3, 6 - retrolateral view. Scale bars: $0.1 \mathrm{~mm}$.

Рис. 1-6. Пальпы самцов Habrocestum latifasciatum (Simon, 1868) (1-3; из Турции, Анталия, Кемер) и H. shulovi Prószyński, 2000 (4-6; из Турции, Намрун): 1, 4 - вид спереди-латерально; 2, 5 - вид снизу; 3, 6 - вид сзади-латерально. Масштаб 0,1 мм.

ARS; 1 (LMNM), no exact locality and date; $30^{7} \sigma^{7}$ (NHMW), Akpehir, Sültandağ, 30.04-7.05.1960, FR; 1 + (NHMW), Finibe, 1.05.1964, FR; $1 \mathrm{O}^{7}$ (NHMW), Kaş, 24.04.1964, FR; $10^{7}$ (MHNG), Phaselis, nr.Tekirova, 30.05.1993, PH. — SYRIA: $2 O^{7} \sigma^{\top}$ (NMPC), NW Syria, castle Qalaat al Kahf nr. Qadmus, 5-7.04.2001, MR.
DIAGNOSIS. This species is most similar to $H$. shulovi Prószyński, 2000 known from Levant and Turkey (see below), from which it can readily be distinguished by the different conformation of its copulatory organs (cf. Figs 1-3, 7-8 and 4-5, 11-12): viz., the 


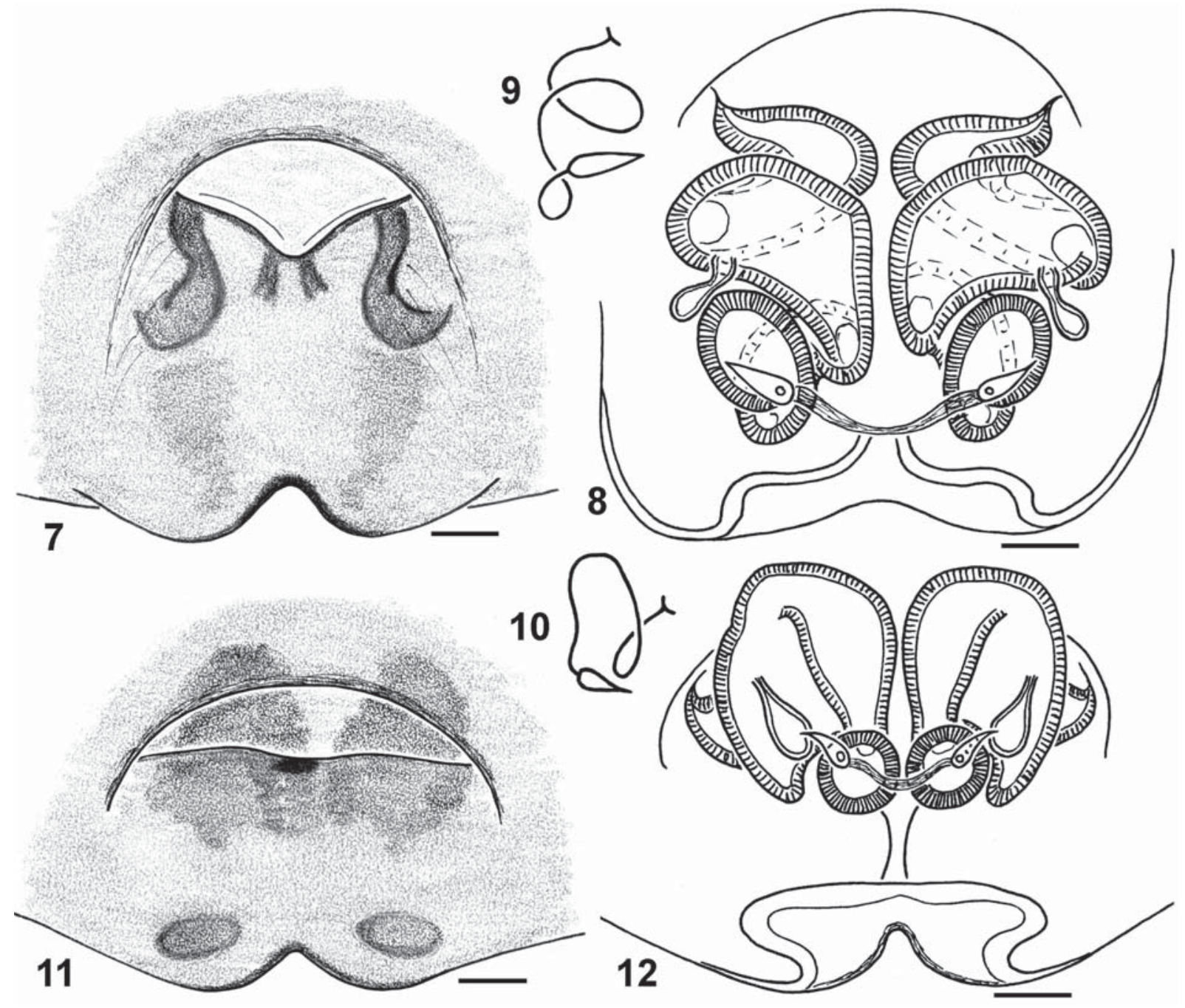

Figs 7-12. Female copulatory organs of Habrocestum latifasciatum (Simon, 1868) (7-9; Turkey, Antalya, Kemer) and H. shulovi Prószyński, 2000 (11-12; Turkey, Namrun): 7, 11 - epigyne, ventral view; 8, 12 - spermathecae, dorsal view; 9, 10 - diagrammatic course of the insemination ducts. Scale bars: $0.1 \mathrm{~mm}$.

Рис. 7-12. Пальпы самцов Habrocestum latifasciatum (Simon, 1868) (7-9; Турция, Анталия, Кемер) и H. shulovi Prószyński, 2000 (11-12; Турция, Намрун): 7, 11 - эпигина, вид снизу; 8, 12 - сперматека, вид сверху; 9, 10 - схематический ход семепроводов. Масштаб 0,1 мм.

longer, bending embolus (straight in $H$. shulovi); the $\mathrm{V}$-shaped rear edge of the epyginal atrium (straight in H. shulovi); and the much stronger and wider insemination ducts.

COMMENTS. Metzner [1999: 61] did not have the samples containing both sexes of this species collected together and therefore mismatched the males from Turkey with the female from Greece. The correct female of $H$. latifasciatum collected together with the male is described here for the first time.

DISTRIBUTION. This seems to be an eastern Mediterranean species, recorded from Greece (both mainland and islands) [Metzner, 1999], eastward throughout Turkey [Karol, 1967; Metzner, 1999; Coșar et al., 2014; present data] to the Near East (Israel, Lebanon and Syria) [Prószyński, 2003; present data]. New record for Syria.

DESCRIPTION. MALE (Turkey, Antalya, Kemer). Measurements. Carapace 3.20 long, 2.40 wide and 1.85 high at PLE. Ocular area 1.53 long, 2.05 wide anteriorly and 1.88 wide posteriorly. Diameter of AME 0.58. Clypeus height 0.25 , chelicera length 1.00. Abdomen 2.55 long, 2.20 wide. Length of leg segments: I: $2.10+$ $1.20+0.78+1.25+0.75 ;$ II: $1.75+0.95+1.05+0.80+$ 0.50 ; III: $2.60+1.10+1.45+1.30+0.60$; IV: $1.80+$ $0.85+1.00+1.25+0.60$. Leg spination. Leg I: Fm d 0-1-1-4; Pt pr 0-1-0; Tb pr 1-0, v 1-2-2ap; Mt pr 1-1ap, v 2-2ap. Leg II: Fm d 0-1-2-5; Pt pr 0-1-0; Tb pr 1-1-1, v 1-2-2ap; Mt pr 1-1ap, v 2-2ap. Leg III: Fm d 0-1-2-4; Pt pr ad rt 0-1-0; Tb d 1-0-0, pr and rt 1-1-1, v 1-0-2ap; 
Mt pr 1-0-2p, rt and v 2-0-2ap. Leg IV: Fm d 0-1-1; Pt pr and rt 0-1-0; Tb d 1-0-0, pr 0-1-1, rt 1-1-1, v 2-12ap; Mt d 2-0, pr 1-1, rt 1-0, v 3-2ap. Coloration. Carapace dark brown, densely covered with brown adpressed hairs, with almost black eye field; there is a wide longitudinal stripe widening anteriorly and running across the eye field and over the thorax (see Metzner [1999: table 26]); there are two large patches of white scales on each side of the carapace, beneath PMEs; clypeus orange-brown, with sparse white and black hairs; eyes of the first row surrounded by long and dense sand-coloured scales. Sternum yellow, covered with white hairs. Maxillae and labium yellow, with white apexes. Chelicerae red-brown. Abdomen: dorsum brownish, with a wide transverse white band anteriorly and a large white inverted triangular figure in the rear half of the dorsum (see Metzner [1999: table $26]$ ); sides and venter yellow, tinged with grey. Booklung covers light yellow; spinnerets grey. Leg I: Fm yellow, with a black prolateral longitidunal stripe; Pt, $\mathrm{Tb}$ and Mt brownish yellow dorsally and black ventrallt and laterally (on both sides); Mt yellow. Legs IIIV yellow, with brownish patches, especially desen on $\mathrm{Pt}, \mathrm{Tb}$ and Mt. Palps brownish yellow, with dense dorsal bunches of long white hairs on Fm (their tips only), Pt and Tb; palpal structure as in Figs 1-3; tibial apophysis short, cone-shaped; bulbus triangle, with the bent proximal end directed median; embolus long and bending.

FEMALE (Turkey, Antalya, Kemer). Measurements. Carapace 3.35 long, 2.55 wide and 1.50 high at PLE. Ocular area 1.48 long, 2.00 wide anteriorly and 1.95 wide posteriorly. Diameter of AME 0.58. Clypeus height 0.15 , chelicera length 0.95 . Abdomen 3.15 long, 2.80 wide. Length of leg segments: I: $1.70+0.95+$ $1.00+0.75+0.60 ;$ II: $11.65+1.00+0.90+0.80+$ 0.55 ; III: $2.50+1.20+1.45+1.35+0.60 ;$ IV: $0.75+$ $0.80+1.20+1.15+0.70$. Leg spination. Leg I: Fm d 0-1-1-3; Pt pr and rt 0-1-0; Tb pr 1-1, v 1-2-2ap; Mt pr 1-1ap, v 2-2ap. Leg II: Fm d 0-1-1-5; Pt pr and rt 0-1-0; Tb pr 1-1, v 1-1-2ap; Mt pr 1-1ap, v 2-2ap. Leg III: Fm d 0-1-2-4; Pt pr ad rt 0-1-0; Tb d 1-0-0, pr 1-1-1, v 2ap; Mt pr 1-0-2p, rt and v 2-0-2ap. Leg IV: Fm d 0-1-1-1; Pt pr and rt 0-1-0; Tb d 1-0-0, pr 1-1-1, v 1-0-2ap; Mt pr 1-1-2ap, rt 2-1-2ap, v 2-0-2ap. Coloration as in the male, but lighter (mostly yellow to sand-coloured) and also differs as follows: there is no white longitudinal stripe on carapace; colour pattern of the dorsum (anterior transverse stripe and inverse triangular figure) pale yellow and pooly marked; legs I and palps entirely yellow; clypeus yellow, with long whote hairs. Epigyne and spermathecae as in Figs 7-9; the wide epigynal pocket presents; the epigynal atrium shallow, with the $\mathrm{V}$-shaped rear edge; the spermathecae with strong and wide insemination ducts making an appearance of multichambered structure; accessory gland well-developed and prominent.
Habrocestum shulovi Prószyński, 2000

Figs 4-6, 10-12.

Habrocestum shulovi Prószyński, 2000: 245, figs 48-51 (D+; holotype + from the Oxford University Museum of Natural History, UK; not examined). 2015].

For a compete set of taxonomic references see WSC

MATERIAL. TURKEY: $1 \Im^{\top}, 2$ 2 (NHMW) \& $1 \Im^{7}$ (ZMUM), Namrun, forest, 12.05-5.06.1964, FR; 19 (NHMW), Eski, Anamuryum, 16.05.(no year), coll.?; 1 + (LMNM), Narlıuyu (36 $26^{\prime}$ $\left.04^{\prime \prime} \mathrm{N}, 34^{\circ} 06^{\prime} 44^{\prime \prime} \mathrm{E}\right), 15.07 .1992$, CF; 1 \% (ZMUM), Nemrut, Dagi, 1.07.2004, LJ.

DIAGNOSIS. This species is most similar to $H$. latifasciatum known from the E Mediterranean (see above), from which it can readily be distinguished by the different conformation of its copulatory organs (cf. Figs 4-6, 11-12 and 1-3, 7-8): viz., the shorter, straight embolus (bending in $H$. latifasciatum); the straight, transverse rear edge of the epyginal atrium (V-shaped in H. latifasciatum); and the much shorter and narrower insemination ducts.

DISTRIBUTION. Israel and Turkey [Prószyński, 2000, 2003; present data]. New record for Turkey.

DESCRIPTION. MALE (Turkey, Namrun). Measurements. Carapace 2.70 long, 2.10 wide and 1.55 high at PLE. Ocular area 1.35 long, 1.90 wide anteriorly and 1.70 wide posteriorly. Diameter of AME 0.55 . Clypeus height 0.33 , chelicera length 0.88 . Abdomen 2.10 long, 1.00 wide. Length of leg segments: I: $2.00+$ $1.10+1.50+1.15+0.85 ;$ II: $1.50+0.90+0.95+0.75+$ 0.55 ; III: $2.40+1.00+1.25+1.25+0.55$; IV: $1.70+$ $0.75+0.90+1.25+0.55$. Leg spination. Leg I: Fm d 0-1-1-4; Pt pr 0-1-0; Tb pr 1-1, v 1-2-2ap; Mt pr 1-01ap, v 2-2ap. Leg II: Fm d 0-1-2-4; Pt pr 0-1-0; Tb pr 1-1-1, rt 1-0, v 1-2-2ap; Mt pr 1-1ap, v 2-2ap. Leg III: Fm d 0-1-2-3; Pt pr ad rt 0-1-0; Tb d 1-0-0, pr and rt 11-1, v 1-0-2ap; Mt pr 1-0-2p, rt and v 2-0-2ap. Leg IV: Fm d 0-1-1-1; Pt pr and rt 0-1-0; Tb d 1-0-0, pr and rt 1-1-1, v 1-0-2ap; Mt pr 1-0-2ap, rt and v 2-0-2ap. Coloration. Carapace red-brown, densely covered with brownish adpressed hairs and with patches of dense brown hairs above ALEs; there is a wide longitudinal stripe widening anteriorly and running across the eye field and over the thorax; clypeus orange, with sparse whitish hairs; eyes of the first row surrounded by long and dense white/yellow scales. Sternum yellow, covered with white hairs. Maxillae and labium yellow, with white apexes. Chelicerae red-brown, with yellowish tips. Abdomen: dorsum brownish grey, with a wide transverse white band anteriorly and a large white inverted triangular figure in the rear half of the dorsum; sides and venter yellow, tinged with grey. Book-lung covers light yellow; spinnerets grey. All legs yellow, but Fm, Pt and $\mathrm{Tb}$ of the first legs with black ventral longitudinal stripe. Palps brownish yellow, with dense dorsal bunches of long white hairs on Fm (their tips only), Pt and Tb; palpal structure as in Figs 4-6; tibial apophysis short, cone-shaped; bulbus triangle, with the bent proximal end directed mediad; embolus long and straight. 
FEMALE (Turkey, Namrun). Measurements. Carapace 3.40 long, 2.65 wide and 2.05 high at PLE. Ocular area 1.55 long, 2.13 wide anteriorly and 2.00 wide posteriorly. Diameter of AME 0.63. Clypeus height 0.30 , chelicera length 1.25 . Abdomen 4.50 long, 3.75 wide. Length of leg segments: I: $1.80+1.05+1.05+$ $0.75+0.60$; II: $1.75+1.10+1.00+0.90+0.60$; III: $2.90+1.30+1.60+1.15+0.70 ;$ IV: $2.05+1.00+$ $1.15+1.45+0.65$. Leg spination. Leg I: Fm d 0-1-1-3; Pt pr and rt 0-1-0; Tb pr 1-1, v 1-2-2ap; Mt pr 1-1ap, v 2-2ap. Leg II: Fm d 0-1-1-5; Pt pr and rt 0-1-0; Tb pr 11, v 1-1-2ap; Mt pr 1-1ap, v 2-2ap. Leg III: Fm d 0-12-4; Pt pr ad rt 0-1-0; Tb d 1-0-0, pr 1-1-1, v 2ap; Mt pr 1-0-2p, rt and v 2-0-2ap. Leg IV: Fm d 0-1-1-1; Pt pr and rt 0-1-0; Tb d 1-0-0, pr 1-1-1, v 1-0-2ap; Mt pr 11-2ap, rt 2-1-2ap, v 2-0-2ap. Coloration as in the male, but lighter (mostly yellow) and also differs as follows: there is no white longitudinal stripe on carapace; colour pattern of the dorsum (anterior transverse stripe and inverse triangular figure) pale yellow and pooly marked; legs I and palps entirely yellow. Epigyne and spermathecae as in Figs 10-12; the wide epigynal pocket presents; the epigynal atrium shallow, with the straight, transverse rear edge; the insemination ducts $\Lambda$-shaped, with round clearly marked receptacles; accessory gland well-developed and prominent.

Heliophanus fuerteventurae Schmidt et Krause, 1996 Figs 13-19.

Heliophanus fuerteventurae Schmidt et Krause, 1996: 269 figs 8-9 ( $\sigma^{7}$; holotype $\sigma^{7}$ from Museum für Tierkunde, Dresden, Germany; examined).

TYPE. Holotype $\sigma^{7}$ (Museum für Tierkunde, Ch 00355; Fig. 15) from [Spain], 'Kanaren: El Jable/Fuenteventura', 8.12.1993, M. Schmidt; the right palp of the holotype, which was illustrated by Schmidt \& Krause [1996: fig. 9], is missing, the right palp and the left first leg are detached.

MATERIAL. SPAIN: $4 \sigma^{7}, 1$ (MHNG), the Canaries, Fuerteventura, below Parque Holandes (entrance to Corralejo et Puerto del Rosario), nr. the sea, small bushes, 20 m a.s.1., 17.11.1998, CL; $1 \sigma^{7}$ (MHNG), the Canaries, Fuerteventura, Vallebron (S of La Oliva), dry bushes and palm trees, c. $250 \mathrm{~m}$ a.s.1., 16.11.1998, CL; $1 \sigma^{7}$ (MHNG), the Canaries, Fuerteventura, nr. Lajares (SW of Corralejo), small bushes, c. $80 \mathrm{~m}$ a.s.1., 15.11.1998, CL.

DIAGNOSIS. This species belongs to the decoratus group [sensu Wesołowska, 1986] and is most similar to H. ibericus Wesołowska, 1986 known from the single male from mainland Spain. The males of these species can easily be separated by the shape of the femoral apophysis, which is sharpened in H. ibericus and bifurcated in $H$. fuerteventurae, and of the embolus, which is slightly bent at its tip in H. ibericus and hook-shaped in $H$. fuerteventurae (cf. Figs 13, 15 with figs 585-588 in Wesołowska [1986]). Of the related species from the decoratus group, the female of $H$. fuerteventurae is most similar to that of $H$. agricoloides Wunderlich, 1987 from the Canaries (cf. Figs 102-103), but can be readily distinguished by the shape of the deeper epigynal pocket and by the thinner insemination ducts (Figs 18-19). See also below the 'Comments' under $H$. agricoloides.
COMMENTS. In the original description, Schmidt \& Krause [1996: fig. 9] mistakenly illustrated the embolic division of the male palp by showing a bifurcated tip of the embolus, which is not the case (cf. Figs 13, 15).

DISTRIBUTION. Restricted to the Fuerteventura island of the Canaries [Schmidt, Krause, 1996; present data].

DESCRIPTION. MALE (from Fuerteventura, Parque Holandes). Measurements. Carapace 1.38 long, 0.98 wide and 0.68 high at PLE. Ocular area 0.69 long, 0.83 wide anteriorly and 0.97 wide posteriorly. Diameter of AME 0.29. Clypeus height 0.04, chelicera length 0.41. Abdomen 1.55 long, 0.95 wide. Length of leg segments: I: $0.69+0.38+0.43+0.38+0.30$; II: $0.58+$ $0.33+0.35+0.31+0.25$; III: $0.68+0.33+0.38+$ $0.40+0.29 ;$ IV: $0.78+0.48+0.50+0.53+0.38$. Leg spination. Legs I and II: Fm d 0-1-1-2; Tb pr 0-1, v 11/2; Mt v 2-2ap. Leg III: Fm d 0-1-1-2; Tb pr and rt 01-1, v 1ap; Mt pr and rt 1-0-2ap, v 1ap. Leg IV: Fm d 0-1-1-2; Tb pr 1-1, rt 1-1-1, v 2ap; Mt pr, rt and v 1-02ap. Coloration. Carapace yellowish brown, with darker (brown) eye field and black around eyes; entire carapace covered with white/transparent scales. Strenum yellow, tinged with brown and covered with white hairs. Labium, maxillae and chelicerae light yellow-brown. Abdomen: dorsum yellow-brown, with a wide transverse stripe at its anterior edge and two pairs of large white spots (as illustrated by Schmidt \& Krause [1996: fig. 8]); sides yellow-brown; venter yellow. Book-lung covers yellow, spinnerets brown. All legs yellow, but Fm I and Tb I with brown pro- and retrolateral sides. Palps yellow, tinged with brown, their structure as in Figs 13-17; femoral process singular, but bifurcated at its tip and with a sharp knob at its base; ventral tibial apophysis narrow and straight; retrolateral tibial apophysis straight, spine-like; bulbus pear-chaped; embolus hook-shaped, bent mediad.

FEMALE (from Fuerteventura, Parque Holandes). Measurements. Carapace 1.80 long, 1.23 wide and 0.80 high at PLE. Ocular area 0.83 long, 1.03 wide anteriorly and 1.18 wide posteriorly. Diameter of AME 0.34. Clypeus height 0.05 , chelicera length 0.50 . Abdomen 1.73 long, 1.15 wide. Length of leg segments: I: $0.80+$ $0.48+0.50+0.40+0.35 ;$ II: $0.73+0.38+0.43+0.38+$ 0.30 ; III: $0.83+0.48+0.50+0.53+0.35$; IV: $1.03+$ $0.48+0.65+0.70+0.40$. Leg spination. Legs I and II: Fm d 0-1-1-1; Tb pr 0-1/2, v 1-1; Mt v 2-2ap. Leg III: Fm d 0-1-1-1; Tb pr 0-1, rt 1-1, v 1ap; Mt pr, rt and v 12ap. Leg IV: Fm d 0-1-1-1; Tb pr 1-1, rt 1-1-1, v 1-02ap; Mt pr 2-0-2ap, rt 1-1-2p, v 2ap. Coloration as in the male but lighter (light yellow); carapace densely covered with white scales; dorsum light sandy-coloured, with the anterior transverse white stripe and two pairs of white spots poorly marked; all legs and palps light yellow. Epigyne and spermathecae as in Figs 18-19; epigynal plate notched at its rear edge; anterior, transverse epigynal pocket procurved; insemination ducts narrow, directed towards the epigastric furrow; receptacles large, transeversely-orientated. 

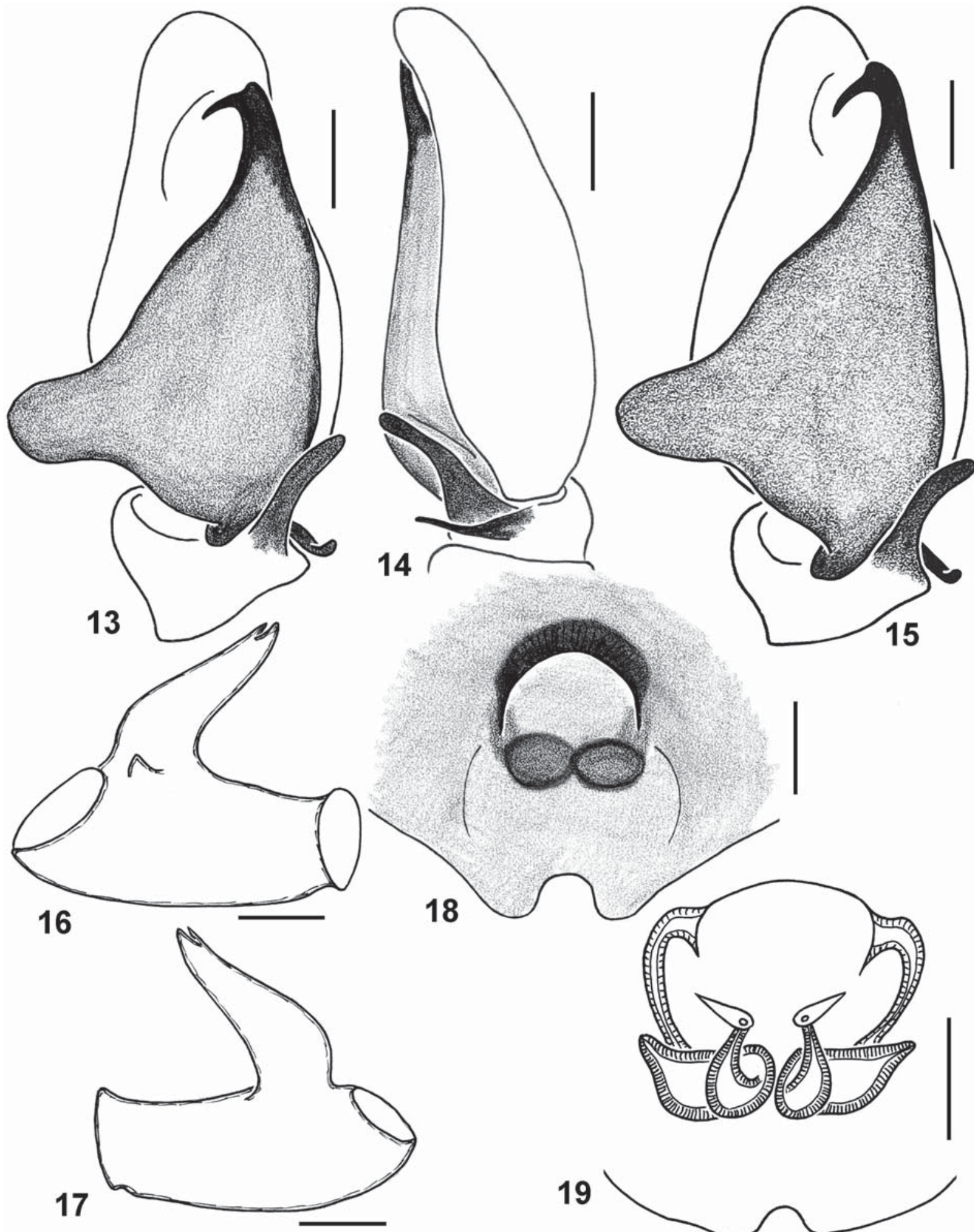

18

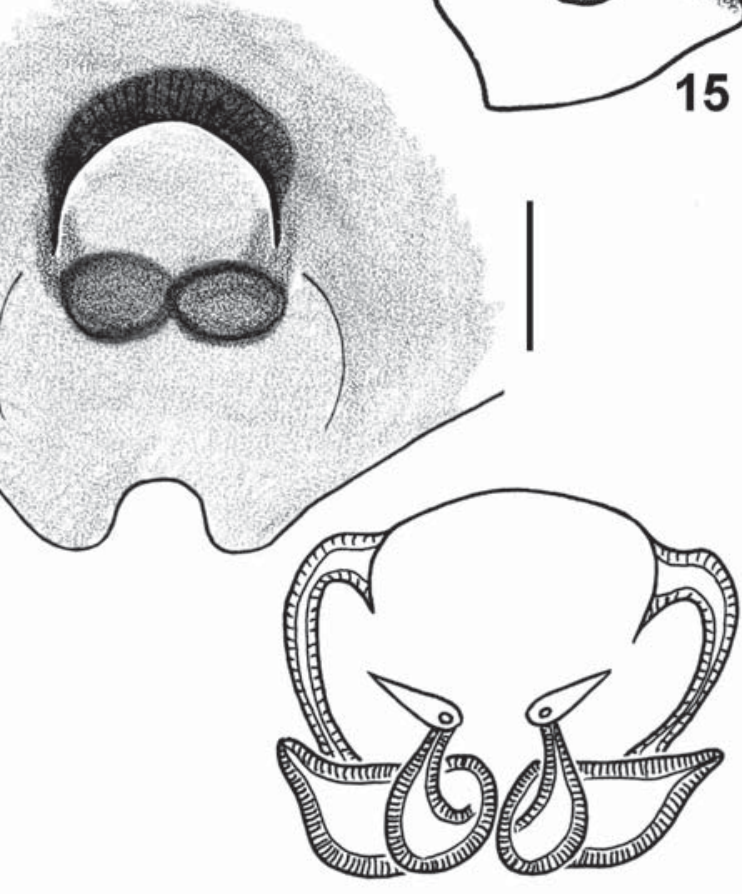

19

Figs 13-19. Copulatory organs of Heliophanus fuerteventurae Schmidt et Krause, 1996 (13-14, 16-19 — Fuerteventura, Parque Holandes; 15 - the holotype): 13, 15 - male palp, ventral view; 14 - ditto, retrolateral view; 16 - male palpal femur, prolatearal view; 17 - ditto, retrolateral view; 18 - epigyne, ventral view; 19 - spermathecae, dorsal view. Scale bars: $0.1 \mathrm{~mm}$.

Рис. 13-19. Копулятивные органы Heliophanus fuerteventurae Schmidt et Krause, 1996 (13-14, 16-19 - Фуертевентура, Голландский парк; 15 - голотип): 13, 15 - пальпа самца, вид снизу; 14 - тоже, сзади-латерально; 16 - бедро пальпы самца, вид спереди-латерально; 17 - тоже, вид сзади-латерально; 18 - эпигина, вид снизу; 19 - сперматека, вид сверху. Масштаб 0,1 мм. 


\section{Heliophanus haymozi sp.n.}

Figs 20-27.

TYPES. Holotype $\sigma^{7}$ (MHNG) from Spain, Zamora [Prov.], nr. Sandin of Carballeda [c. $42.00728^{\circ} \mathrm{N},-6.47932^{\circ} \mathrm{W}$ ], 17.07.1975, PH. - Paratypes: PORTUGAL: $10^{7}, 1$ q (ZMUM), Mértola (c. $\left.37^{\circ} 38^{\prime} \mathrm{N},-7^{\circ} 40^{\prime} \mathrm{W}\right), 27.05 .2003$, PC.

MATERIAL: SPAIN: $1 O^{7}$ (NMPC), Xávia (=Jávea), NP del Mongú, 29.04.2004, LD; $2 O^{\top} \sigma^{\top}$ (NMPC), Valensia, Guadalest (c. $\left.38^{\circ} 41^{\prime} \mathrm{N}, 0^{\circ} 11^{\prime} \mathrm{E}\right), 27.04 .2004, \mathrm{LD}$.

ETYMOLOGY. Dedicated to the collector of the holotype, Mr Pierre-Joseph Haymoz (Geneva, Switzerland).

DIAGNOSIS. The new species is most similar to the Euro-Mediterranean species H. tribulosus Simon, 1868 [cf. Wesołowska, 1986: figs 492-497; Metzner, 1999: table 65]. The male can easily be distinguished from it by the presence of the prominent apical projection of the bulbus (arrowed in Fig. 20), the longer embolus and the singular tip of the femoral process (arrowed in Fig. 24; bifurcated in H. tribulosus). The female can be distinguished by the significantly wider and stronger insemination ducts directed laterad (Fig. 27; the relatively thin insemination ducts directed anteriad in H. tribulosus).

DISTRIBUTION. The Iberian Peninsula (Portugal and Spain) [present data]. Some of the earlier records of H. tribulosus from Spain mentioned in Wesołowska [1986] and Helsdingen [2014] may actually belong to H. haymozi sp.n.

DESCRIPTION. MALE (the holotype). Measurements. Carapace 2.08 long, 1.30 wide and 0.80 high at PLE. Ocular area 0.77 long, 1.03 wide anteriorly and 1.07 wide posteriorly. Diameter of AME 0.34. Clypeus height 0.04 , chelicera length 0.73 . Abdomen 1.85 long, 1.23 wide. Length of leg segments: I: $0.95+0.55+$ $0.65+0.53+0.38 ;$ II: $0.80+0.45+0.48+0.45+$ 0.33 ; III: $0.90+0.40+0.50+0.58+0.35$; IV: $1.03+$ $0.45+0.68+0.73+0.40$. Leg spination. Legs I and II: Fm d 0-1-1-1; Tb pr 0-1, v 1-1; Mt v 2-2ap. Leg III: Fm d 0-1-1-1; Tb pr 0-1, rt 1-1, v 1ap; Mt pr and rt 2ap. Leg IV: Fm d 0-1-1-2; Tb pr 0-1-1, rt 1-1-1, v 1-0-1ap; Mt pr 1-0-2ap, rt 1-1-2ap. Coloration. Carapace redbrown, with dark brown eye field and black around eyes; eye field sparsely covered with white scales. Sternum, labium, maxillae and chelicerae yellowish brown. Abdomen: dorsum yellow-brown, without colour pattern; sides and venter light brownish yellow. Booklung covers yellow, spinnerets yellow-brown. All lesg: Fm brown; $\mathrm{Pt}$ and $\mathrm{Tb}$ brown, but with yellow dorsal sides; Mt and Tr yellow. Palps: Fm and Pt brownish yellow; cymbium and bulbus brown; palpal structure as in Figs 20-25; femoral process is bent at its top half, with a sharp short process at its base; ventral tibial apohysis short and hook-shaped (bent ventrad), and with massive base; bulbus almost square; embolus straight, almost as long as the bulbus.

FEMALE. (the paratype). Measurements. Carapace 2.03 long, 1.35 wide and 0.83 high at PLE. Ocular area 0.90 long, 1.10 wide anteriorly and 1.23 wide posteriorly. Diameter of AME 0.38. Clypeus not marked, chelicera length 0.60. Abdomen 2.90 long, 2.00 wide.
Length of leg segments: I: $0.89+0.58+0.55+0.48+$ 0.38 ; II: $0.83+0.53+0.45+0.48+0.35$; III: $0.95+$ $0.50+0.55+0.60+0.40 ;$ IV: $1.23+0.58+0.83+$ $0.90+0.48$. Leg spination. Legs I: Fm d 0-1-1-1; Tb v 2-2; Mt v 2-2ap. Leg II: Fm d 0-1-1-1, Tb pr 0-1, v 1-1; Mt v 2-2ap. Leg III: Fm d 1-1-2; Tb pr 0-1, rt 1-1, v 10-1ap; Mt pr and rt 2ap, v 1-0-1ap. Leg IV: Fm d 1-1-1; Tb pr 1-1, rt 0-0-2, v 1-0-2ap; Mt pr 1-1-2ap, rt 1-02ap, v 1ap. Coloration. Carapace brown, with black eye field and orange sides, sparsely covered with transparent adpressed scales. Strenum brown, covered with white hairs. Maxillae and labium orgnge, with white tips. Chelicerae orange. Abdomen entirely turquoisegrey, with a white transverse stripe anteriorly and with no colour pattern on the dorsum. Book-lung covers yellow, tinged with grey; spinnerets turquoise-grey. All legs and palps entirely yellow. Epigyne and spermathecae as in Figs 26-27; deep central atrium present; copulatory openings separated and clearly seen at the rear-bottom of the atrium; insemination ducts as wide and lond as receptacula, directed laterad; receptacula bean-shaped.

\section{Heliophanus ramosus Wesołowska, 1986} Figs 28-30, 34-36.

Heliophanus ramosus Wesołowska, 1986: 42-43, figs 503-

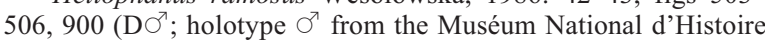
Naturelle, Paris, France; not examined).

MATERIAL. ALGERIA: $1 \sigma^{\top}$ (PCRB), Wilaya Oran, forest Msila, c. $400 \mathrm{~m}$ a.s.1., sieving litter in Quercus suber and among stones and Oxalis, 25.04.1984, RB. - SPAIN: $1 \sigma^{7}, 1$ ( 1 (MVHN), Valencia, Devesa del Saler, fixed dunes, 20.05.2004, DD.

DIAGNOSIS. This species is very similar to $H$. stylifer Simon, 1878 known from Morocco and Algeria [Wesołowska, 1986] (see below), but can reliably be distinguished from it by the bi-ramous femoral process in the males (cf. Figs 29 and 32) and the wider epigynal plate (cf. Figs 34 and 37) and the arrangement of the insemination ducts and receptacles in the females (cf. Figs $35-36$ and 38-39).

DISTRIBUTION. A few localities in Algeria and Spain [Wesołowska, 1986; present data].

DESCRIPTION. MALE, see in Wesołowska [1986]; Figs 28-30.

FEMALE (from Spain, Valencia). Measurements. Carapace 1.95 long, 1.38 wide and 0.80 high at PLE. Ocular area 0.90 long, 1.14 wide anteriorly and 1.27 wide posteriorly. Diameter of AME 0.33. Clypeus height 0.03, chelicera length 0.56. Abdomen 2.95 long, 2.05 wide. Length of leg segments: I: $0.88+0.50+0.53+$ $0.50+0.38$; II: $0.75+0.43+0.48+0.40+0.34$; III: $0.88+0.43+0.50+0.53+0.35 ;$ IV: $1.13+0.53+$ $0.78+0.88+0.40$. Leg spination. Legs I and II: Fm d 0-1-1-1; Tb v 1-1; Mt v 2-2ap. Leg III: Fm d 1-0-1; Tb pr and rt 1-1-1, v 1ap; Mt pr and rt 2ap. Leg IV: Fm d 1-1-2; Tb pr and rt 1-1-1, v 2ap; Mt pr and rt 2ap. Coloration. Carapace brownish, with no colour pattern, eye feld dark brown (almost black). Strenum brown, covered with white hairs. Maxillae and labium brown, with yellow apexes. Chelicerae red-brown. Ab- 

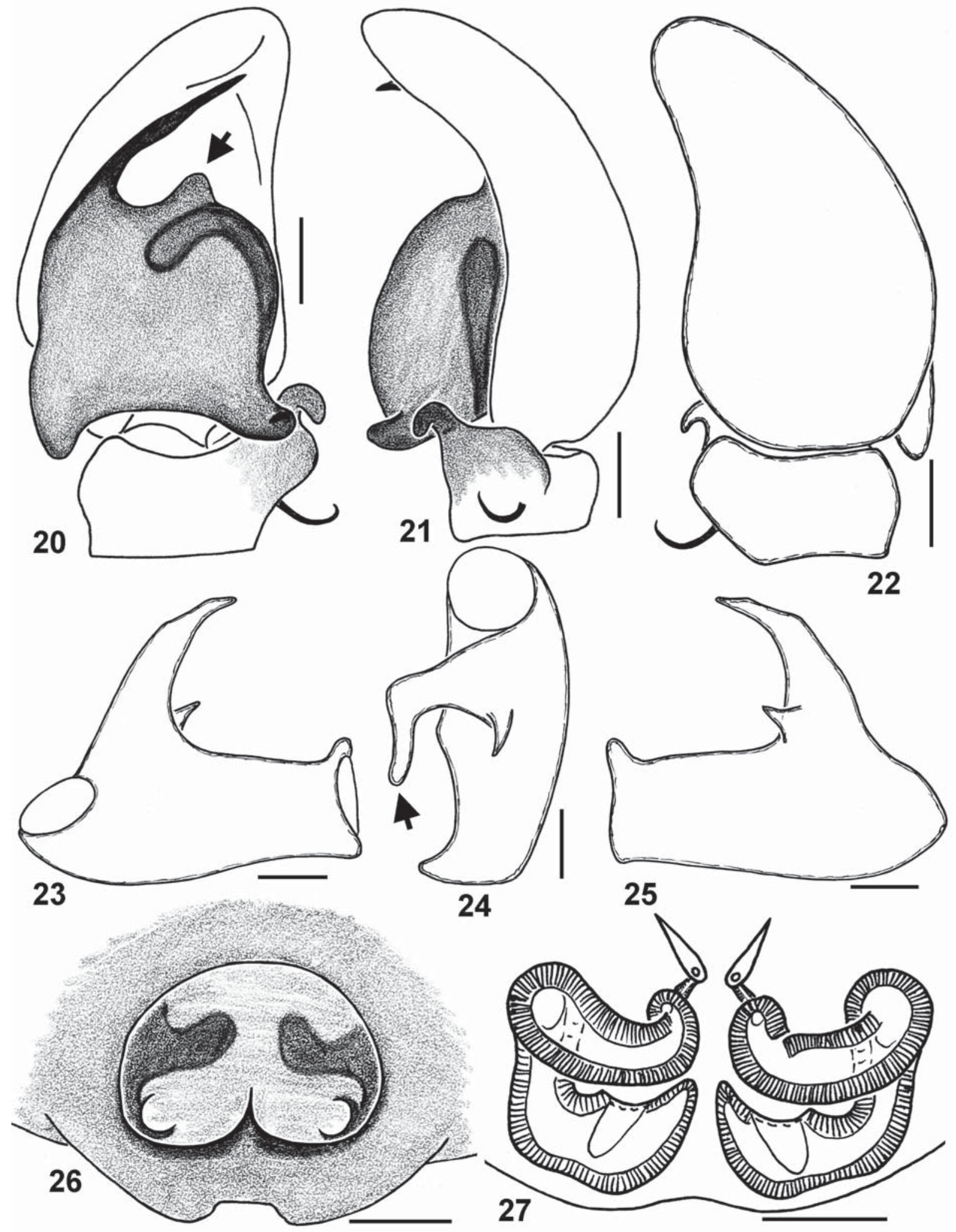

Figs 20-27. Copulatory organs of Heliophanus haymozi sp.n. ( $0^{7}$ - the holotype; +9 - the paratype, Portugal, Mértola): 20 - male palp, ventral view; 21 - ditto, retrolateral view; 22 - ditto, dorsal view; 23 - male palpal femur, retrolatearal view; 24 - ditto, dorsal view; 25 - ditto, prolateral view; 26 - epigyne, ventral view; 27 - spermathecae, dorsal view. Scale bars: $0.1 \mathrm{~mm}$.

Рис. 20-27. Копулятивные органы Heliophanus haymozi sp.n. (о — голотип; 으 — паратип, Португалия, Мертола): 20 — пальпа самца, вид снизу; 21 — тоже, сзади-латерально; 22 - тоже, вид сверху; 23 - бедро пальпы самца, вид сзади-латерально; 24 тоже, вид сверху; 25 - тоже, вид спереди-латерально; 26 - эпигина, вид снизу; 27 - сперматека, вид сверху. Масштаб 0,1 мм. 


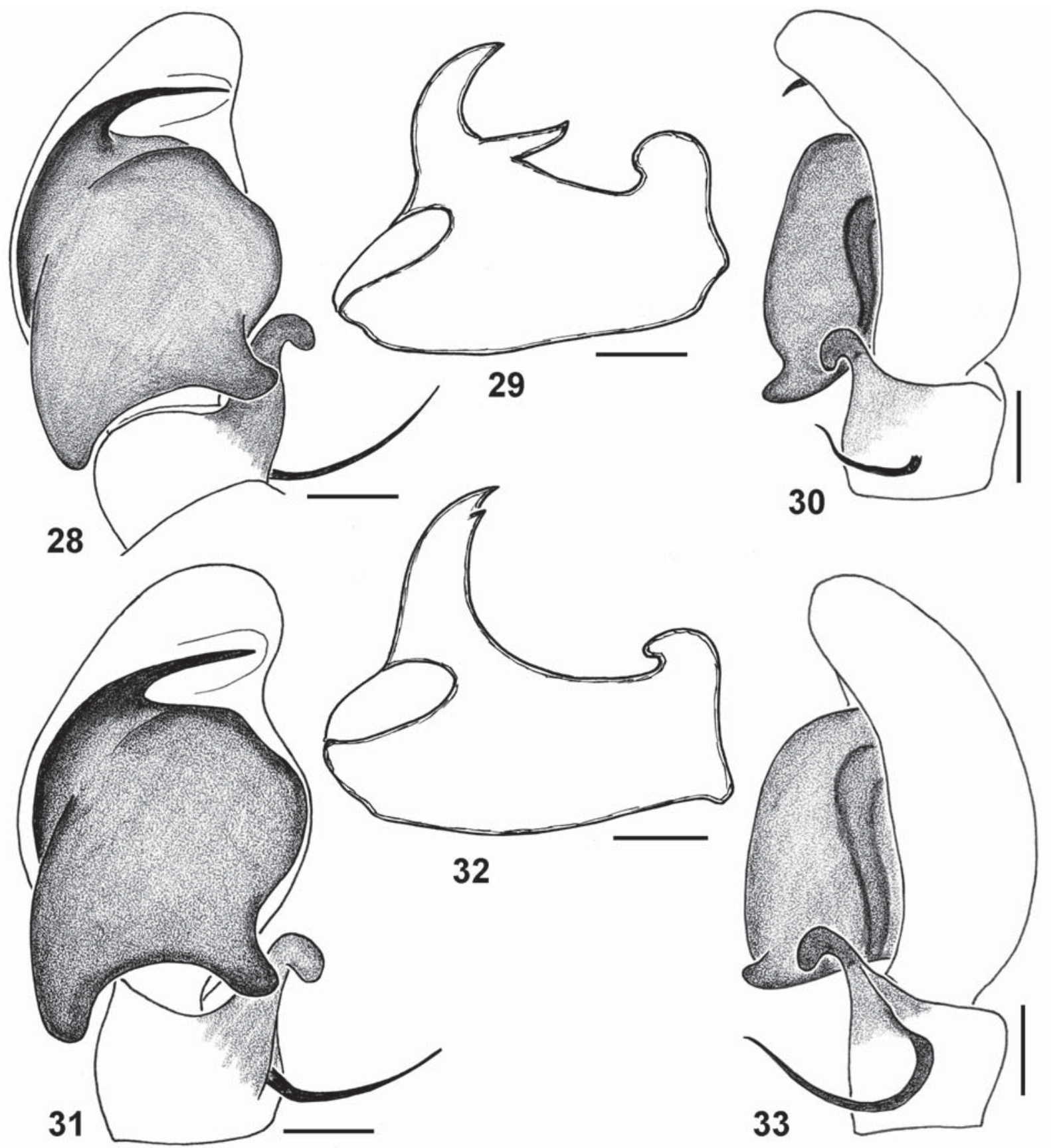

Figs 28-33. Male palps of Heliophanus ramosus Wesołowska, 1986 (28-30; Spain, Valencia) and H. stylifer Simon, 1878 (31-33; Algeria, Wilaya Bejaia): 28, 31 - bulbus, ventral view; 29, 32 - male palpal femur, prolateral view; 30, 33 — bulbus, retrolateral view. Scale bars: $0.1 \mathrm{~mm}$.

Рис. 28-33. Пальпы самцов Heliophanus ramosus Wesołowska, 1986 (28-30; Испания, Валенсия) и H. stylifer Simon, 1878 (3133; Алжир, вилайя Беяха): 28, 31 - вид снизу; 29, 32 - бедро пальпы самца, вид спереди-латерально; 30, 33 - бульбус, вид сзади-латерально. Масштаб 0,1 мм.

domen: dorsum and sides grey-brown, with a narrow transverse white frontal line on dorsum; venter yellowish brown. Book-lung covers and spinnerets light brown. All legs brown with yellow tarsi and darker (dark brown) femora, and with one or two dorsal yellow lines on $\mathrm{Tb}$ and Mt. Palps: coxae, Fm and Pt brown, Tb and Mt contrastingly yellow. Epigyne and spermathecae as in Figs 34-36; epigyne with two wide copulatory orifices facing ahead; short, tube-like insemination ducts and bean-shaped receptacles. 

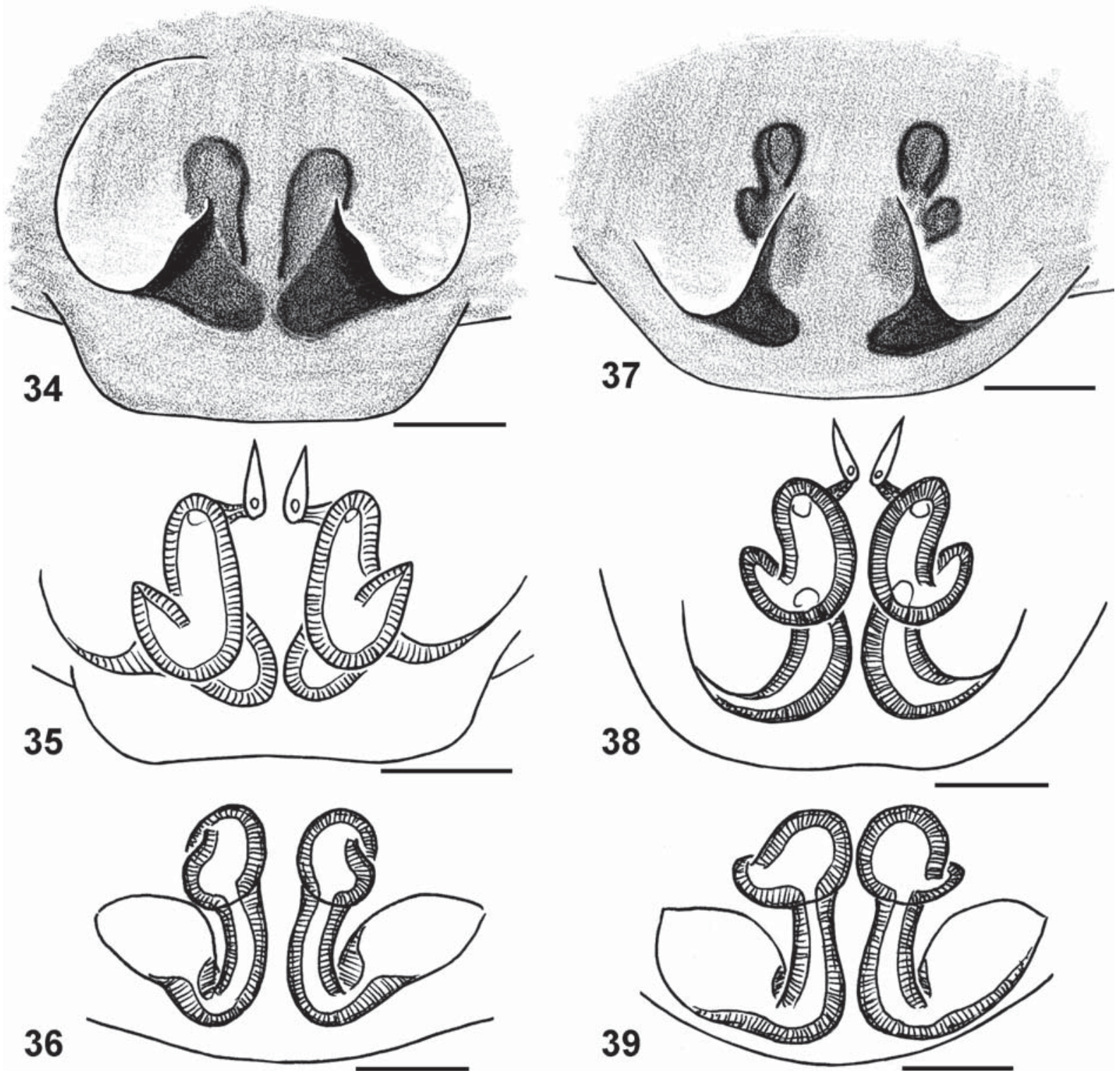

36

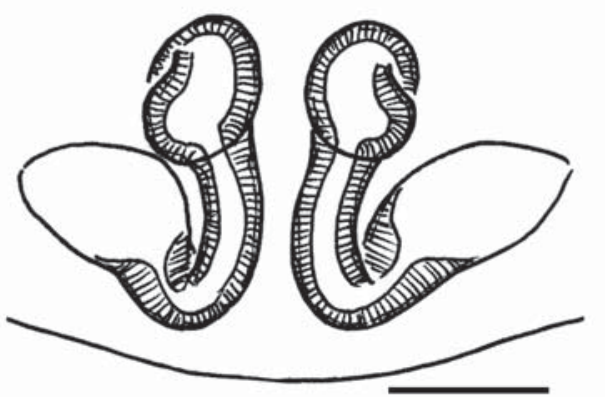

39

Figs 34-39. Female copulatory organs of Heliophanus ramosus Wesołowska, 1986 (34-36; Spain, Valencia) and H. stylifer Simon, 1878 (37-39; Algeria, Wilaya Bejaia): 34, 37 - epigyne, ventral view; 35, 38 - spermathecae, dorsal view; 36, 39 - ditto, rear view. Scale bars: $0.1 \mathrm{~mm}$.

Рис. 34-39. Копулятивные органы самок Heliophanus ramosus Wesołowska, 1986 (34-36; Испания, Валенсия) и H. stylifer Simon, 1878 (37-39; Алжир, вилайет Беяха): 34, 37 - эпигина, вид снизу; 35, 38 - сперматека, вид сверху; 36, 39 — тоже, вид сзади. Масштаб 0,1 мм.

\section{Heliophanus sinaicus sp.n.}

Figs $40-46$.

TYPES: Holotype $0^{7}$ (NHMW) from Egypt, Sinai, Pharaoh's Island (c. $\left.29.46333^{\circ} \mathrm{N}, 34.85944^{\circ} \mathrm{E}\right), 08.1982$, N. Shaumer. Paratypes: 1 o (NHMW) and $1 \sigma^{7}$ (ZMUM), together with the holotype.

The comparative material on $H$. decoratus is given below under 'New faunistic records'.

ETHYMOLOGY. The species is named after the terra typica: Sinai in Egypt.

DIAGNOSIS. H. sinaicus sp.n. belongs to the decoratus group [sensu Wesołowska, 1986] and is most similar to the Mediterranean species $H$. decoratus L. Koch, 1875 and H. konradthaleri Logunov, 2009 from Turkey [see Wesołowska, 1986; Prószyński, 2003; Logunov, 2009b]. It can readily be distinguished from both by the following combination of characters: the hook-shaped embolus and the strong singular femoral apophysis in the males (Figs 41, 43), and the S-shaped spermathecae in the females (Fig. 46).

COMMENTS. One of the females of $H$. decoratus (from Libya) illustrated by Wesołowska [1986: figs 583-584] shows the virtually identical conformation of the spermathecae with those of $H$. sinaicus sp.n. (cf. 

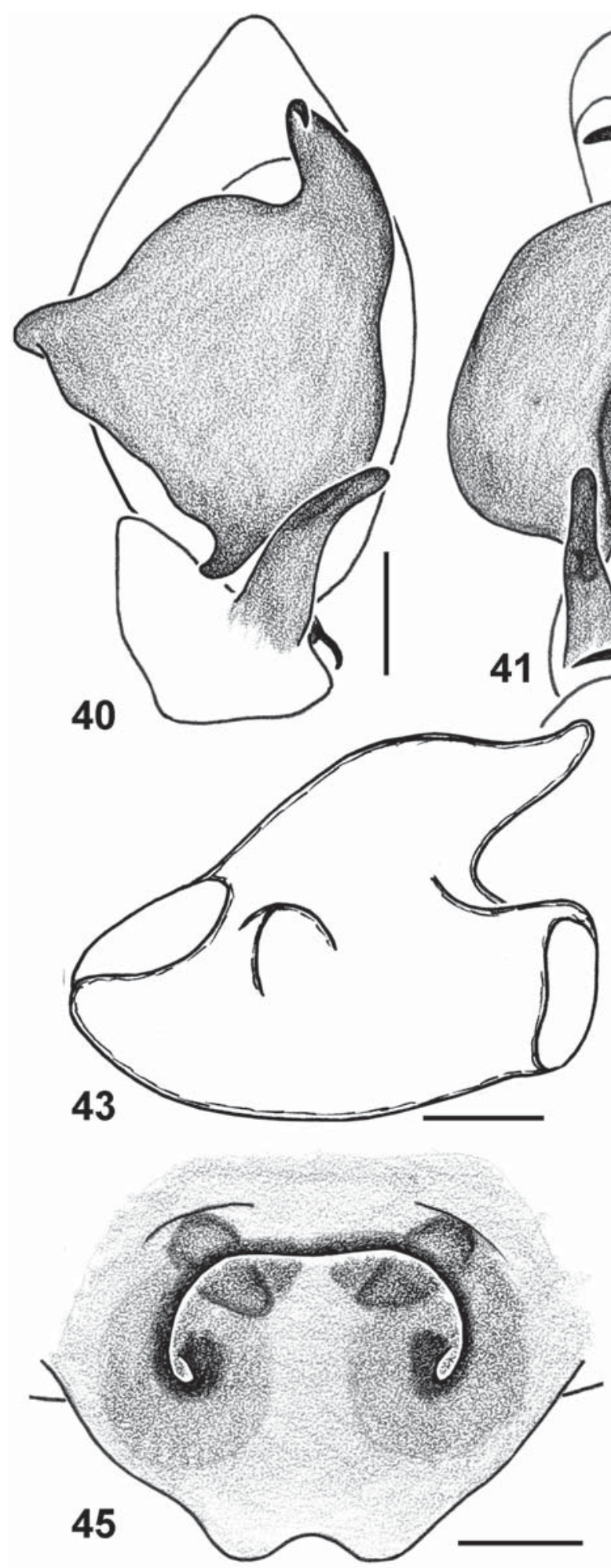
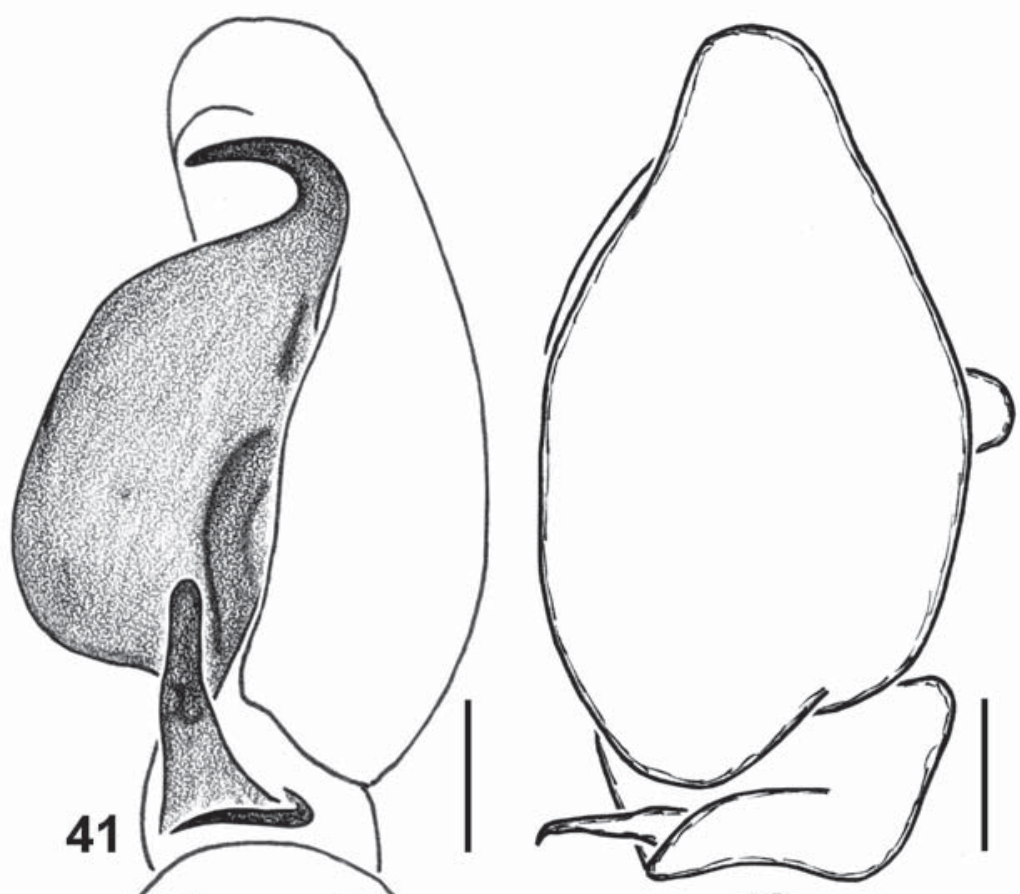

42
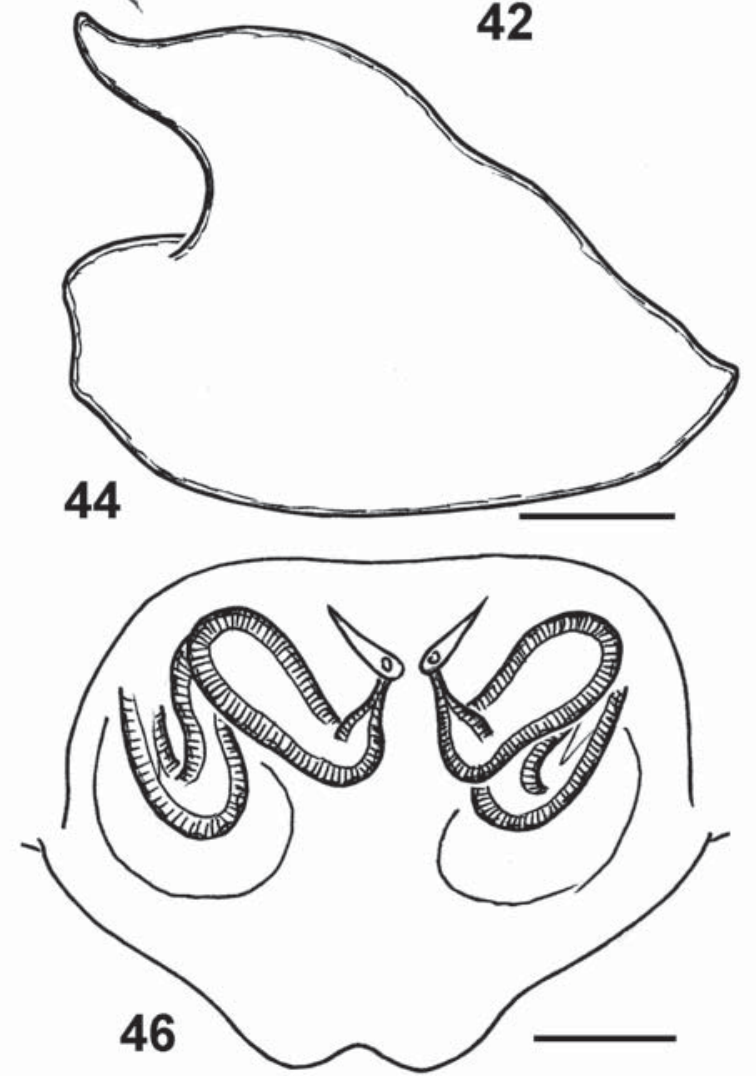

Figs 40-46. Copulatory organs of Heliophanus sinaicus sp.n. ( $0^{7}$ holotype; 9 paratype): 40 - male palp, ventral view; 41 - ditto, retrolateral view; 42 - ditto, dorsal view; 43 - male palpal femur, prolatearal view; 44 - ditto, retrolateral view; 45 - epigyne, ventral view; 46 - spermathecae, dorsal view. Scale bars: $0.1 \mathrm{~mm}$.

Рис. 40-46. Копулятивные органы Heliophanus sinaicus sp.n. (о голотип; ㅇ паратип): 40 — пальпа самца, вид снизу; 41 тоже, сзади-латерально; 42 - тоже, вид сверху; 43 - бедро пальпы самца, вид спереди-латерально; 44 — тоже, вид сзадилатерально; 45 - эпигина, вид снизу; 46 - сперматека, вид сверху. Масштаб 0,1 мм. 
Fig. 46). Yet, Wesołowska [1986: 209] pointed out to the strong variation in the structure of copulatory organs of $H$. decoratus suggesting that the studied series of specimens apparently consisted of a number of closely related species. The discovery of $H$. sinaicus sp.n. is in favour of the latter assumption.

DISTRIBUTION. The type locality: Egypt (Sinai). However, the record of $H$. decoratus from Libya by Wesołowska [1986: fig. 584] seems to belong to this species as well.

DESCRIPTION. MALE (the holotype). Measurements. Carapace 1.63 long, 1.23 wide and 0.78 high at PLE. Ocular area 0.84 long, 1.00 wide anteriorly and 1.09 wide posteriorly. Diameter of AME 0.34. Clypeus height 0.04 , chelicera length 0.53 . Abdomen 1.55 long, 1.05 wide. Length of leg segments: I: $0.78+0.50+$ $0.55+0.45+0.38 ;$ II: $0.70+0.40+0.40+0.38+0.30$; III: $0.76+0.45+0.45+0.45+0.38 ;$ IV: $0.93+0.48+$ $0.60+0.60+0.45$. Leg spination. Leg I: Fm d 0-1-1-2; Tb pr 0-1, v 1-2; Mt v 2-2ap. Leg II: Fm d 0-1-1-2; Tb pr 0-1, v 1-2ap; Mt pr 1ap, v 2-2ap. Leg III: Fm d 0-11-3; Pt rt 0-1-0; Tb pr 1-1, rt 1-1-1, v 1-0-2ap; Mt pr, rt and v 1-0-2ap. Leg IV: Fm d 0-1-1-3; Pt rt 0-1-0; Tb pr 1-1, rt 1-1-1, v 1-0-2ap; Mt pr and rt 1-0-2ap, v 2ap. Coloration. Carapace yellowish orange, sparsely covered with white scales; eye field brownish, with black around eyes. Sternum light yellow, covered with white scales. Labium, maxillae and chelicerae yellowish orange. Abdomen light yellow, tinged wth brownish on dorsum. Book-lung covers light yellow, spinnerets contrastingly brown. All legs light yellow. Femora I with a set of 4 stridulatory bristles situated in their apical parts. Palps yellow, tinged with brown, their structure as in Figs 40-44; femoral process thick and hookshaped; ventral tibial apophysis finger-shaped; bulbus tringle, with strong hook-shaped embolus.

FEMALE (the paratype). Measurements. Carapace 1.73 long, 1.25 wide and 0.75 high at PLE. Ocular area 0.84 long, 1.01 wide anteriorly and 1.16 wide posteriorly. Diameter of AME 0.31. Clypeus height 0.04, chelicera length 0.67 . Abdomen 2.05 long, 1.60 wide. Length of leg segments: I: $0.80+0.48+0.45+0.45+$ 0.33 ; II: $0.70+0.40+0.38+0.35+0.30$; III: $0.83+$ $0.45+0.48+0.53+0.35 ;$ IV: $1.05+0.45+0.68+$ $0.70+0.43$. Leg spination. Legs I and II: Fm d 0-1-11/2; Tb pr 0-1, v 1-1; Mt v 2-2ap. Leg III: Fm d 0-1-12; Pt pr 0-1-0; Tb pr and rt 1-1, v 1ap; Mt pr, rt and v 12ap. Leg IV: Fm d 0-1-1-1; Pt rt 0-1-0; Tb pr 1-1, rt 11-1, v 1-0-2ap; Mt pr, rt and v 1-2ap. Coloration as in the male, but visibly lighter, with eye field contrastingly brown. Femora I with a set of 4 stridulatory bristles situated in their apical parts. Palps entirely light yellow. Epigyne and spermathecae as in Figs 45-46; epigyne with a transverse, omega-shaped ridge; insemination ducts S-shaped; receptacles elongated.

\section{Icius crassipes (Simon, 1868)}

Figs 47-52.

Attus crassipes Simon, 1868: 574 (D $\sigma^{7}$; holotype $\sigma^{7}$ from Muséum National d'Histoire Naturelle, Paris, France; not exam- ined). The type series was re-examined by Alicata \& Cantarella [1994].

For a complete set of taxonomic references see WSC [2015].

MATERIAL. ALGERIA: $1 \sigma^{\top}, 1$ (ZMUM), Wilaya Saida, waterfalls nr. Tifrit on Wadi Tifrit, c. $825 \mathrm{~m}$ a.s.1., moist habitat at the foot of the waterfall, between herbs (Stellaria, Geranium, etc.), 4.05.1984, RB; 1 (PCRB), Boumerdes, Reghalia, c. 10 m a.s.1., pitfall traps in Olea europea orchard, 13.06-30.09.1988, RB; 1 ऽ (PCRB; the specimen is badly damaged), Wilaya Blida, Atlas Blidéen, Chrea E., peak Fertasse, c. $1450 \mathrm{~m}$ a.s.l., pitfall traps in Cedrus forest, 12.04.1987-9.05.1988, RB; 1 o (PCRB), Wilaya Bouira, Tikjda, c. $1400 \mathrm{~m}$ a.s.1., forest and grassland, 11.06.1984, RB; $1 O^{7}$ (ZMUM), Wilaya Tlemcen, SE of Tlemsen, forest Hafir, c. $1350 \mathrm{~m}$ a.s.1., sweeping in mixed forest of Quercus ilex, $Q$. suber, Juniperus sp. and Erica arborea, 6.05.1984, RB.

DIAGNOSIS. The male of I. crassipes can be distinguished by the shape of the medium-sized embolus and the lateral cymbial outgrowth and by the conformation of the tibial apophysis (Figs 48-49); see also Alicata \& Cantarella [1994]. The female of I. crassipes is most similar to that of I. simoni Alicata et Cantarella, 1994 from Algeria [cf. Alicata, Cantarella, 1994: figs 72-73], but can easily be distinguished by the copulatory orifices situated closer to each other (Fig. 51) and the insemination ducts directed mediad (Fig. 52) rather than anteriad as in I. simoni.

DISTRIBUTION. Spain, Algeria and Tunisia [Alicata, Cantarella, 1994; present data].

DESCRIPTION. MALE (from Algeria, Wilaya Saida, waterfalls nr. Tifrit). Measurements. Carapace 2.10 long, 1.48 wide and 0.88 high at PLE. Ocular area 1.03 long, 1.28 wide anteriorly and 1.30 wide posteriorly. Diameter of AME 0.43. Clypeus not marked, chelicera length 0.83 . Abdomen 2.35 long, 1.50 wide. Length of leg segments: I: $1.13+0.60+0.80+0.58+0.40$; II: $0.90+0.50+0.58+0.53+0.38$; III: $1.00+0.50+$ $0.59+0.70+0.43 ;$ IV: $1.15+0.58+0.78+0.80+$ 0.43. Leg spination. Leg I: Fm d 0-1-1-2; Tb pr 0-1, v 2-2; Mt v 2-2ap. Leg II: Fm d 0-1-1-2; Tb pr 0-1, v 1-1; Mt v 2-2ap. Leg III: Fm d 0-1-1-2; Tb pr 0-1, rt 1-1, v 1ap; Mt pr and rt 2ap. Leg IV: Fm d 0-1-1; Tb pr 0-1, rt 1-1, v 1ap; Mt pr and rt 2ap. Coloration. Carapace reddish brown, with black around eyes, covered with white appressed scales. Strenum yellow, tinged with brown and covered with white hairs. Maxillae, labium and chelicerae pink-yellow; cheliceral crest low and poorly developed (Fig. 50). Abdomen: dorsum and sides yellowish brownish with white specks and a white marginal stripe around the edges of dorsum; venter yellow. Book-lung covers light yellow, spinnerets brownish. Legs I yellowish brownish, darker than other legs and its femora slightly stronger than those of other legs. Legs II-IV entirely yellow, with pro- and retrolateral sides of Fm and Pt tinged with brown. Femora I with a set of stridulatory bristles situated in their apical parts. Palps pink-yellow, covered with white hairs, their structure as in Figs 47-50; tibial apophysis mediumsized, cone-shaped, with a clearly pronounced sharp basal tooth; cymbium with strong, finger-shaped (as seen in lateral view) lateral cymbial process [sensu Logunov, 1996]; bulbus ovoid, narrowing towards its 

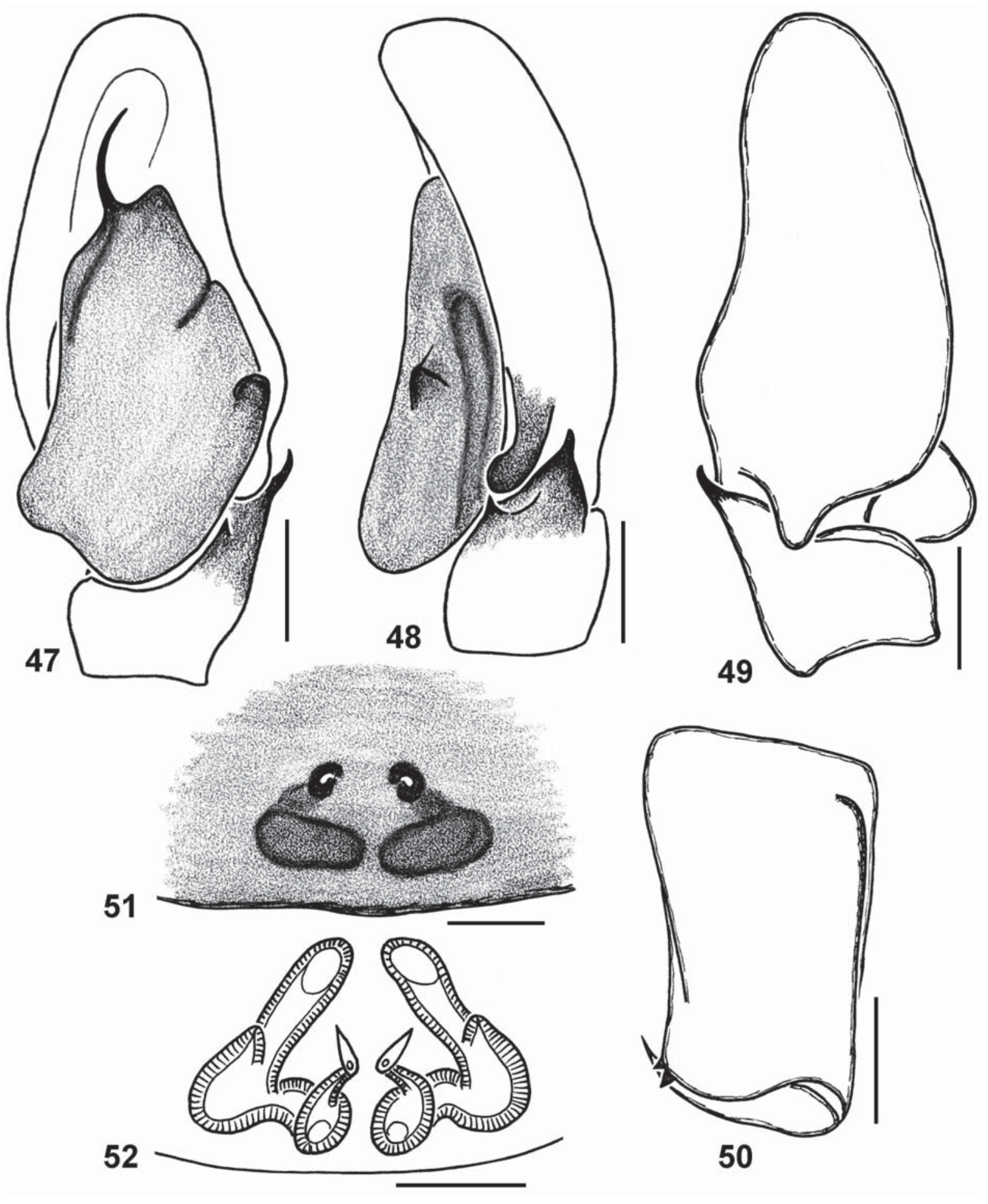

Figs 47-52. Copulatory organs and chelicera of Icius crassipes (Simon, 1868) (Algeria, Wilaya Saida, waterfalls nr. Tifrit): 47 - male palp, ventral view; 48 - ditto, retrolateral view; 49 - ditto, dorsal view; 50 - male chelicera, dorsal view; 51 - epigyne, ventral view; 52 - spermathecae, dorsal view. Scale bars: $0.1 \mathrm{~mm}$.

Рис. 47-52. Копулятивные органы и хелицера Icius crassipes (Simon, 1868) (Алжир, вилайет Саида, водопад возле Тифрита): 47 - пальпа самца, вид снизу; 48 - тоже, сзади-латерально; 49 - тоже, вид сверху; 50 - хелицера самца, вид сверху; 51 эпигина, вид снизу; 52 - сперматека, вид сверху. Масштаб 0,1 мм. 
apical end; embolus medium-sized (compared to other Icius species), spine-shaped.

FEMALE (from Algeria, Wilaya Saida, waterfalls nr. Tifrit). Measurements. Carapace 2.80 long, 2.03 wide and 1.00 high at PLE. Ocular area 1.23 long, 1.55 wide anteriorly and 1.70 wide posteriorly. Diameter of AME 0.50. Clypeus not marked, chelicera length 1.00. Abdomen 5.10 long, 3.15 wide. Length of leg segments: I: $1.38+0.80+0.88+0.73+0.48$; II: $1.25+$ $0.70+0.73+0.65+0.43$; III: $1.35+0.65+0.75+$ $0.90+0.53 ;$ IV: $1.68+0.80+1.08+1.13+0.53$. Leg spination. Legs I: Fm d 0-1-1-2; Tb v 2-2-1; Mt v 22ap. Leg II: Fm d. 0-1-1-2, Tb v 1-1, Mt v 2-2ap. Leg III: Fm d 0-1-1-2; Tb pr and rt 0-1, v 1ap; Mt pr and rt 2ap, v 1ap. Leg IV: Fm d 0-1-1-1; Tb pr and rt 0-1, v 10-1ap; Mt pr and rt 2ap, v 1-0-1ap. Coloration as in the male, but visibly lighter and differs in the following characters: clypeal edge densely covered with white hairs; dorsum without the marginal white stripe around its edges. Palps entirely yellow. Epigyne and spermathecae as in Figs 51-52; epigyne with two small but clearly visible copulatory openings; insemination ducts run towards the epigastric furrow; proximal and distal sections of the receptacles only slightly wider than the insemination ducts.

\section{Icius insolitus Alicata et Cantarella, 1994 Figs 53-62.}

Icius insolitus Alicata et Cantarella, 1994: 127, f. 5, 11, 16, 47, 62-68 (D $\sigma^{7}$; holotype $\sigma^{7}$ from Muséum National d'Histoire Naturelle, Paris, France; not examined)

MATERIAL. ALGERIA: $3 \sigma^{7} \sigma^{\gamma}, 1$ (PCRB), Wilaya Gulema, Ain Regada, c. 600 m a.s.1., herbs along Wadi Zenati, 22.11.1989, $\mathrm{RB}$.

DIAGNOSIS. The male of $I$. insolitus differs from other Icius species in having the strongly-developed cheliceral crest (Figs 57-60), the relatively small embolus (Figs 53-54) and in the shape of tibial apophysis (Fig. 55); see also Alicata \& Cantarella [1994: 127] for more diagnostic characters for the male. The female of I. insolitus differs from those of all other Icius species in having the insemination ducts running dorsad and therefore poorly seen in dorsal view (Fig. 62); the shape of proximal and distal chambers of receptacles are also diagnostic.

DISTRIBUTION. Algeria only [Alicata, Cantarella, 1994; present data].

COMMENTS. The males of I. insolitus demonstrate a very strong variation both in the body size (in more than 1.5 times; see the measurements given below) and in the development of cheliceral crest (Figs 57-60). Yet, the embolus remains of the same size, despite size differences in the bulbus length (Figs 5354).

Recently, Wunderlich [2011: 329] described a new Icius species: I. lamellatus Wunderlich, 2011 from Italy and Portugal. The main diagnostic characters from the suggested related species I. insolitus were the shape of cheliceral crest and the smaller embolic size. However, I. lamellatus, as was illustrated by Wunderlich
[2011: figs 1-9], seems to be identical with the westMediterranean - south-European I. subinermis Simon, 1937, both in the conformation of its male palp (cf. Alicata, Cantarella [1994: figs 52-53]) and the size and shape of the cheliceral crest (cf. Jäger [1995: fig. 1]). Therefore, the name I. lamellatus is likely to be a junior synonym of I. subinermis; the matter requires further attention in the future.

DESCRIPTION. MALE. Measurements. Carapace 2.15-3.00 long, 1.56-2.25 wide and 0.80-1.45 high at PLE. Ocular area 1.05-1.38 long, 1.25-1.63 wide anteriorly and $1.33-1.78$ wide posteriorly. Diameter of AME 0.44-0.50. Clypeus not marked, chelicera length 0.88-1.68. Abdomen 2.30-3.75 long, 1.45-2.25 wide. Length of leg segments: I: $1.23-2.13+0.75-1.30+$ $0.88-1.70+0.70-1.28+0.45-0.63$; II: $0.93-1.38+$ $0.50-0.80+0.60-0.93+0.58-0.88+0.38-0.48$; III: $1.03-1.40+0.53-0.75+0.60-0.88+0.68-1.00+$ $0.43-0.55$; IV: $1.20-1.60+0.58-0.80+0.80-1.13+$ $0.80-1.15+0.40-0.50$. Leg spination. Leg I: Fm d 01-1-2; Tb v 0-1-1; Mt v 2-2ap. Leg II: Fm d 0-1-1-2; Tb pr 0-1, v 0-1; Mt v 2-2ap. Leg III: Fm d 0-1-1-2; Tb pr and rt 0-1, v 1ap; Mt pr and rt 2ap, v 1ap. Leg IV: Fm d 0-1-1-1; Tb pr 0-1, rt 0-1-1, v 1-0-2ap; Mt pr and rt 2ap, v 1ap. Coloration. Carapace yellowish brownish, covered with black hairs, with black around eyes and with white elongated spots of white scales along fovea, behind PLEs and below PMEs; there are also white marginal piping of scales and white transverse stripe of scales in front of eye field. Sternum yellow, tinged with brown and covered with white hairs. Labium and maxillae pink-yellow. Chelicerae yellowish brown, their dorsal (anterior) sides darker (brown to dark brown), and bear a narrow, elongated cheliceral crest (Figs 57-60). Abdomen: dorsum and sides yellowish brownish, with a white marginal stripe around the edges of dorsum (see Alicata \& Cantarella [1994: fig. 47]); venter yellow. Book-lung covers yellow, spinnerets brownish. Legs I stronger and darker than others, brown with yellow Tr. Legs II-IV entirely yellow. Femora I with a set of stridulatory bristles situated in their apical parts. Palps yellow, tinged with brown, with brown bulbus; palp structure as in Figs 53-56; tibial apophysis short, cone-shaped, with a poorly visible sharp basal tooth; cymbium with finger-shaped (as seen in lateral view) lateral cymbial process [sensu Logunov, 1996]; bulbus ovoid, narrowing towards its apical end; embolus relatively short, spine-shaped.

FEMALE. Measurements. Carapace 2.50 long, 1.80 wide and 0.95 high at PLE. Ocular area 1.15 long, 1.45 wide anteriorly and 1.60 wide posteriorly. Diameter of AME 0.50. Clypeus not marked, chelicera length 0.80 . Abdomen 2.68 long, 1.75 wide. Length of leg segments: I: $1.25+0.78+0.85+0.58+0.43$; II: missing; III: $1.20+0.58+0.63+0.80+0.45 ;$ IV: $1.43+0.68+$ $1.00+1.00+0.48$. Leg spination. Legs I: Fm d 0-1-12; Tb pr and v 0-1; Mt v 2-2ap. Leg II: missing. Leg III: Fm d 0-1-1-2; Tb pr 0-1, rt 0-1-1, v 1ap; Mt pr and rt 2p, v 1ap. Leg IV: Fm d 0-1-1-1; Tb pr and rt 0-1, v 1ap; Mt pr and rt 2p, v 1ap. Coloration as in the male, 

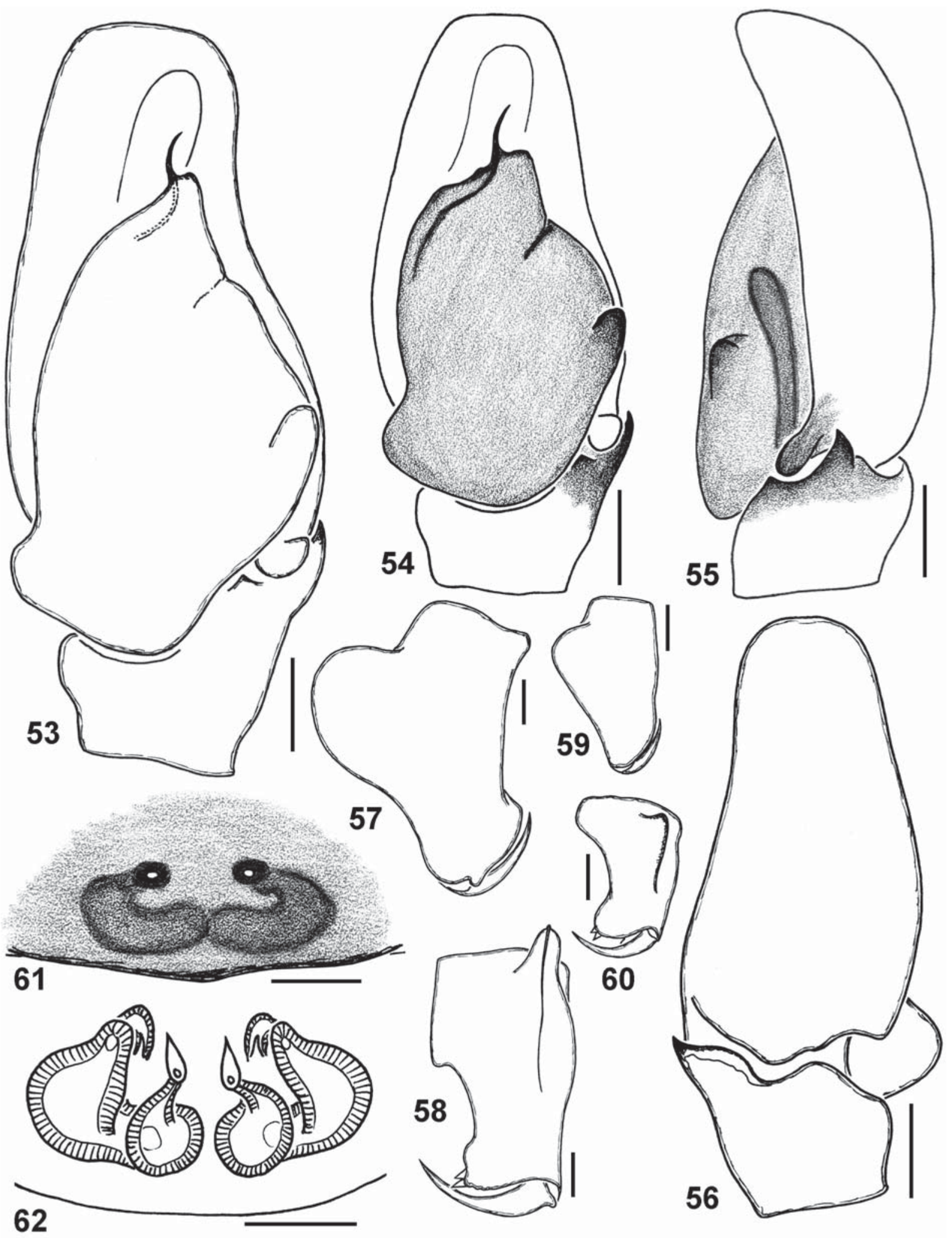

Figs 53-62. Copulatory organs and chelicerae of Icius insolitus Alicata et Cantarella, 1994 (Algeria, Wilaya Gulema, Ain Regada): 53, 54 - male palp, ventral view; 55 - ditto, retrolateral view; 56 - ditto, dorsal view; 57, 59 - male chelicera, lateral view; 58, 60 - ditto, dorsal view; 61 - epigyne, ventral view; 62 - spermathecae, dorsal view. Scale bars: $0.1 \mathrm{~mm}$.

Рис. 53-62. Копулятивные органы и хелицеры Icius insolitus Alicata et Cantarella, 1994 (Алжир, вилайет Гулема, Айн Регада): 53, 54 - пальпа самца, вид снизу; 55 — тоже, вид сзади-латерально; 56 - тоже, вид сверху; 57, 59 - хелицера самца, вид сбоку; 58, 60 - тоже, вид сверху; 61 - эпигина, вид снизу; 62 - сперматека, вид сверху. Масштаб 0,1 мм. 

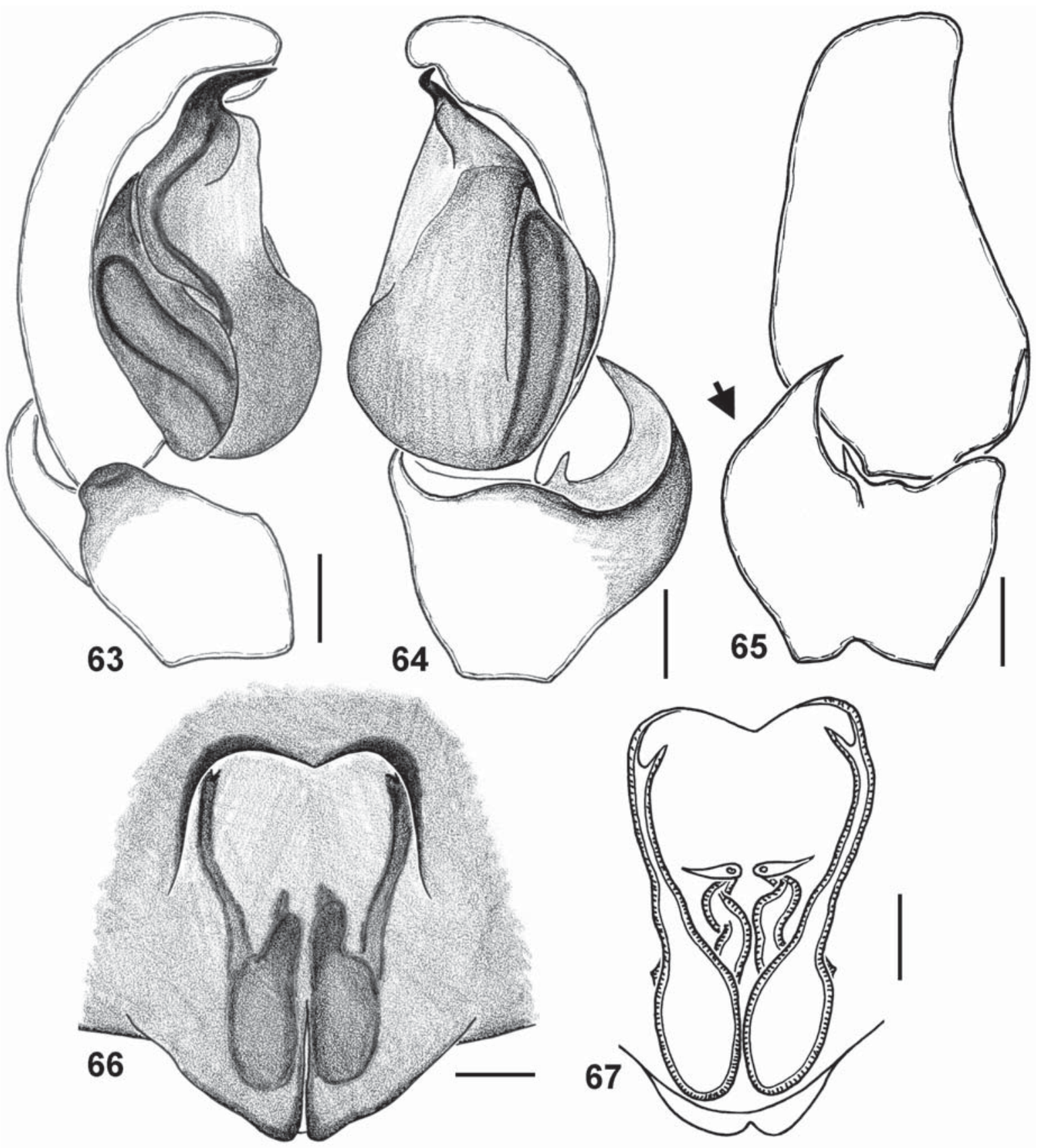

Figs 63-67. Copulatory organs of Salticus confusus Lucas, 1846 (Spain, Valensia, Guadalest): 63 — male palp, prolateral view; 64 ditto, ventral view; 65 - ditto, dorsal view; 66 - epigyne, ventral view; 67 - spermathecae, dorsal view. Scale bars: $0.1 \mathrm{~mm}$.

Рис. 63-67. Копулятивные органы Salticus confusus Lucas, 1846 (Испания, Валенсия, Гуадалест): 63 - пальпа самца, вид спереди-латерально; 64 - тоже, вид снизу; 65 - тоже, вид сверху; 66 - эпигина, вид снизу; 67 - сперматека, вид сверху. Масштаб 0,1 мм.

but visibly lighter (light yellow) and differ in the following aspects: carapace yellow with brown eye field and with no white elongated spots; dorsum yellow, with two wide brown longitudinal stripes; all legs and palps entirely light yellow. Epigyne and spermathecae as in Figs 61-62; epigyne with two small but clearly visible copulatory openings; insemination ducts run dorsally and therefore are not visible in dorsal view; proximal sections of the receptacles pear-shaped and larger than the distal sections.

\section{Salticus confusus Lucas, 1846}

Figs 63-71.

Salticus confusus Lucas, 1846: 163, pl. 8, f. 2 (D ${ }^{7}+$; the type series from Muséum National d'Histoire Naturelle, Paris, France; not examined). 
For a complete set of taxonomic references see WSC [2015].

MATERIAL. ALGERIA: 1 $\sigma^{7}$ (PCRB), Wilaya Blida, Atlas Blidéen, Meurdja, c. $950 \mathrm{~m}$ a.s.l., pitfall traps in the forest of Pinus halepensis, 15.06.1982, RB; 1 o (PCRB), Wilaya Blida, Atlas Blidéen, Djebel Ferroukha, Ghellaï, c. $1350 \mathrm{~m}$ a.s.l., pitfall traps in the cedrus forest, 20.06.1987-9.05.1988, RB; $1 \sigma^{7}$ (PCRB), Wilaya Blida, Atlas Blidéen, Chrea O., peak E. Abdelkader, c. 1520 m a.s.1., pitfall traps in Cedrus forest with Berberis bushes, 20.06.19879.05.1988, RB; 1 (PCRB), Wilaya Blida, Atlas Blidéen, Djebel Mouzaia, c. $1250 \mathrm{~m}$ a.s.1., under stones along the lake, 14.05.1988, RB; 1 O (PCRB), Wilaya Tipasa, Douaouda, Wadi Mazafran, c. 50 $\mathrm{m}$ a.s.1., pitfall traps in Olea orchard, 19.12.1986-26.02.1988, RB; 1 + (PCRB), Wilaya Bejaia, E of the mouth of Wadi Daas, c. 5 m a.s.l., under stones and on bushes on the shore, 22.05.1988, RB; $1 O^{7}, 1$ (PCRB), Wilaya Tipasa, Sidi Fredj, c. $10 \mathrm{~m}$ a.s.l., beating off branches in Pinus halepensis forest, 12.06.1987, RB; $1 \sigma^{\top}$ (PCRB), same locality, pitfall traps in Olea stands, 10.12.1986-20.12.1987, RB; 1 $\sigma^{7}$ (PCRB), Wilaya Tipasa, Douaouda, Wadi Mazafran, 50 m a.s.1., glades in forest of Populus alba, 16.12.1986-26.02.1988, RB. PORTUGAL: $15 \mathrm{O}^{7} \mathrm{O}^{7}, 22$ 90 (FMNH), Mértola (c. $37^{\circ} 38^{\prime} \mathrm{N},-7^{\circ}$ $\left.40^{\prime} \mathrm{W}\right), 28.05 .2003, \mathrm{PC} ; 1 \mathrm{O}^{\top}$ (FMNH), Algodôr (c. $37^{\circ} 45^{\prime} \mathrm{N},-7^{\circ}$ $48^{\prime} \mathrm{W}$ ), pitfall traps, 21.05.2003, PC; $2 \sigma^{7} \sigma^{\top}, 1$ ( 1 (FMNH), Paúl do Boquilobo Biosphere Reserve (c. 39 $23^{\prime} \mathrm{N},-8^{\circ} 32^{\prime} \mathrm{W}$ ), 28.05-5.06.

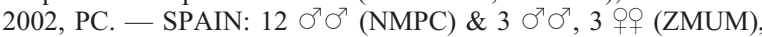
Valensia, Guadalest (c. $38^{\circ} 41^{\prime} \mathrm{N}, 0^{\circ} 11^{\prime} \mathrm{E}$ ), 27.04.2004, LD; 1 O (NMPC), Deltebre, 26.04.2004, LD; $10 \sigma^{\top} \sigma^{\top}, 2$ 90 (NMPC), NP del'Albufera, 30.04.2004, LD; $2 \sigma^{7} \sigma^{7}$ (NMPC), Guardamar, del Segura, 28.04.2004, LD; 1 ○ (NMPC), Besalú, 2.05.2004, LD; 1 ठ (NMPC), Sagunt (=Sagunto), 26.04.2004, LD; 2 ○ $\sigma^{7}$ (SMNH), Montserrat, 1.05.2004, LD; 4 ठ $\sigma^{7}$ (SZMN), Xávia (=Javeá), NP del Mongú, 29.04.2004, LD. — GREECE: 1 ○ (PCJK), Crete, Imbros Gorge, beating, 9.04.2002, J. Van Keer; $1 \sigma^{7}$ (PCJK), Crete, Fran Gokastell, 10.04.2002, J. Van Keer

DIAGNOSIS. The male of $S$. confusus is most similar to those of two poorly known species: $S$. conjonctus (Simon, 1868) from Italy and France and $S$. modicus (Simon, 1875) from France, illustrated by Simon [1937]. From both species, S. confusus can be distigushed by the presence of a sharp tooth at the base of tibial apophysis and by the hook-shaped embolus (Figs $64,68,70)$. The female of $S$. confusus is most similar to that of $S$. zebraneus (C.L. Koch, 1837) [Metzner, 1999: table 82], from which it differs in having the longer and narrower insemination ducts and a different shape of the epigynal plate (Figs 66-67).

COMMENTS. As I have been unable to re-examine the type series, the present identification is based on the illustrations provided by Simon [1937: figs 19061907] and also on the fact that to date the species has been known from the Iberian Peninsula and Corsica only [Helsdingen, 2014].

The two studied males of $S$. confusus from Crete are characterised by the visibly narrower tibial apophysis, as compared to the Algerian specimens (arrowed in Figs 65, 69). Therefore, it is likely that the Cretan population may belong to a different, closely related species. The matter needs further attention, when more material of both sexes has been collected from Crete.

DISTRIBUTION. A Mediterranean species that is more common in the western Mediterranean: from NW Africa (Algeria) and the Iberian Peninsula (Spain and Portugal) in the west, to Corsica and Crete in the east [Simon, 1937; Helsdingen, 2014; present data]. New record for Greece (Crete).
DESCRIPTION. MALE (from Spain, Valensia, Guadalest). Measurements. Carapace 1.78 long, 1.15 wide and 0.68 high at PLE. Ocular area 0.89 long, 1.00 wide anteriorly and 1.01 wide posteriorly. Diameter of AME 0.34. Clypeus not marked, chelicera length 0.77. Abdomen 1.63 long, 1.00 wide. Length of leg segments: I: $0.85+0.50+0.53+0.48+0.30$; II: $0.73+$ $0.40+0.43+0.38+0.28 ;$ III: $00.80+0.43+0.45+$ $0.48+0.35 ;$ IV: $0.98+0.43+0.58+0.55+0.40$. Leg spination. Leg I: Fm d 0-1-1-2; remaining segments are spineless. Leg II: Fm d 0-1-2; remaining segments are spineless. Leg III: Fm d 1-0; Mt 6ap. Leg IV: Fm d 1-0; Tb rt 0-1, v 2ap; Mt 6ap. Coloration. Carapace brown: eye field dark brown, with a wide, white transverse band of white scales in its front (above AMEs); there are two white scaly patches behind PLEs and white marginal band of white scales. Sternum yellowish brown, covered with white hairs. Maxillae, labium and chelicerae yellowish brown. Abdomen: dorsum and sides dark grey-brown, with three trasverse white bands of white scales of dorsum; venter lighter, brown yellowish. Book-lung covers and spinnerets yellow brownish. All legs yellowish with brownish sides of $\mathrm{Pt}, \mathrm{Tb}$ and Mt, with legs I darker than the others. Palps yellow, with brownish bulbus, their structure as in Figs $63-65,68-71$; the tibial apophysis strongly bent mediad, and with the sharp tooth situated at its base of tibial apophysis; the hook-shaped embolus.

FEMALE (from Spain, Valensia, Guadalest). Measurements. Carapace 2.15 long, 1.34 wide and 0.88 high at PLE. Ocular area 0.99 long, 1.19 wide anteriorly and 1.24 wide posteriorly. Diameter of AME 0.39. Clypeus not marked, chelicera length 0.57. Abdomen 2.33 long, 1.50 wide. Length of leg segments: I: $0.91+$ $0.58+0.53+0.40+0.38 ;$ II: $0.83+0.35+0.45+0.40+$ 0.30 ; III: $0.95+0.50+0.50+0.53+0.45 ;$ IV: $1.15+$ $0.60+0.68+0.70+0.43$. Leg spination. Legs I and II: Fm d 0-1-1-2; Mt v 1ap. Leg III: Fm d 0-1-1-3; Tb rt 01; Mt v 6ap. Leg IV: Fm d 0-1-1-1; Tb rt 0-1, v 1ap; Mt v 6ap. Coloration as in the male but lighter, especially light grey abdomen and light yellow legs; only apical tips of Fm, Pt, Tb and proximal parts of Mt are tinged with brown. Palps light yellow. Epigyne and spermathecae as in Figs 66-67; the epigynal plate extends over the epigastric furrow, with a narrow, median cut in its rear part and two narrow epigynal wings anteriorly; insemination ducts narrow; receptacles ovoid with tubelike terminal sections.

Salticus ressli sp.n.

Figs 72-74.

TYPE. Holotype $\sigma^{7}$ (NHMW, 4185) from Turkey, [Mersin], Namrun (c. $\left.37^{\circ} 10^{\prime} 13^{\prime \prime} \mathrm{N}, 34^{\circ} 36^{\prime} 04^{\prime \prime} \mathrm{E}\right)$, pine forest, 12.05-5.06.1964, FR.

ETHYMOLOGY. The species is dedicated to Franz Ressl (1924-2011), the zoologist from the Natural History Museum in Vienna (Austria) who collected the holotype. 

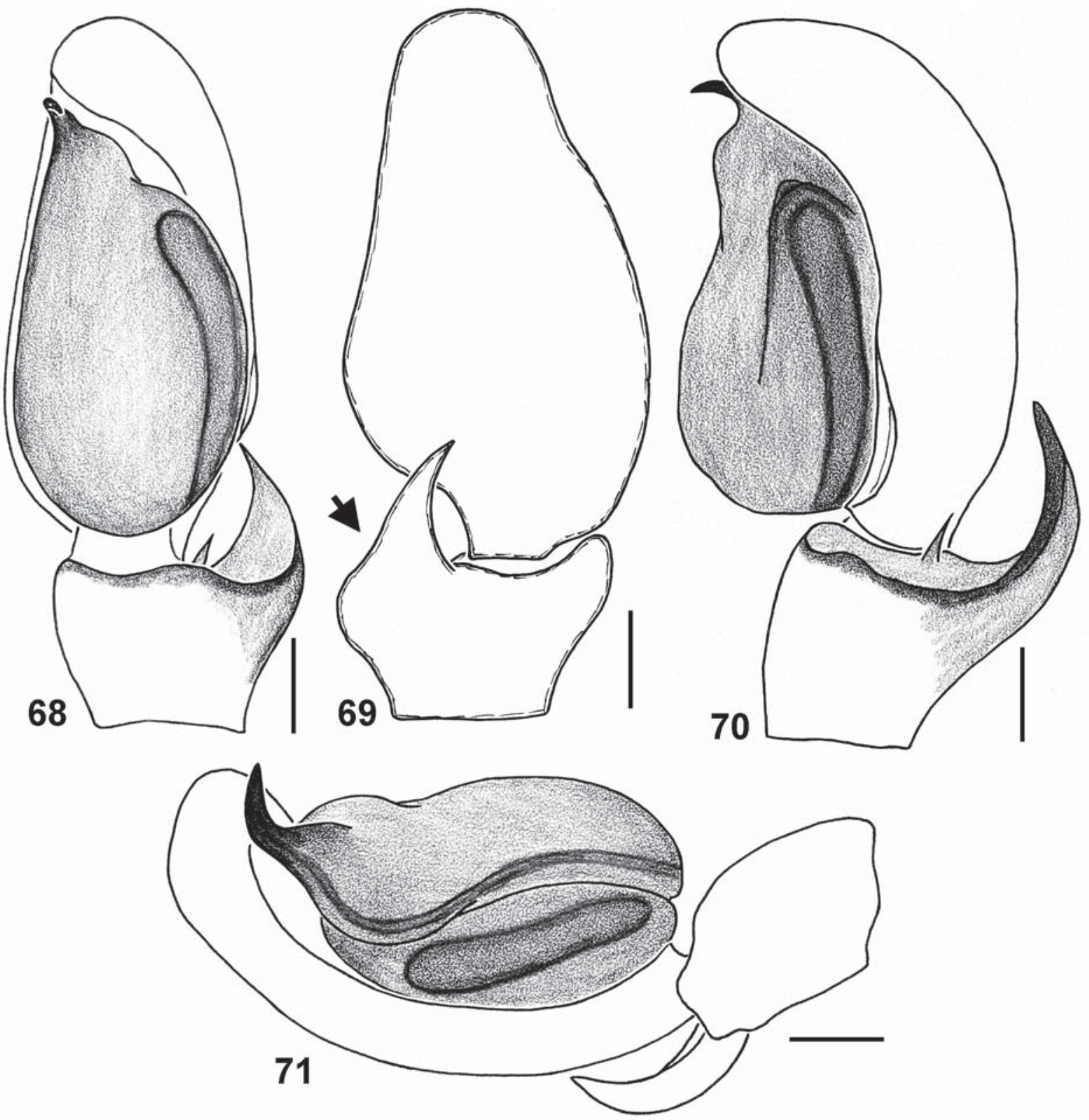

Figs 68-71. Male palp of Salticus confusus Lucas, 1846 (Greece, Crete): 68 - ventral view; 69 - dorsal view; 70 - retrolateral view; 71 - prolateral view. Scale bars: $0.1 \mathrm{~mm}$.

Рис. 68-71. Пальпа самца Salticus confusus Lucas, 1846 (Греция, Крит): 68 - вид снизу; 69 - вид сверху; 70 - вид сзадилатерально; 71 - вид спереди-латерально. Масштаб 0,1 мм.

DIAGNOSIS. The new species is most similar to the Mediterranean-Central Asian species S. tricinctus (C.L. Koch, 1846), but can readily be distinguished by the position of the embolus directed more anteriad than in S. tricinctus (cf. Figs 72 and 109, 111), the shape of tibial apophysis having as if an 'excavated' tip and the absence of a small tooth at the base of tibial apophysis (cf. Figs 73 and 110).

DISTRIBUTION. The type locality only.

DESCRIPTION. MALE (the holotype). Measurements. Carapace 2.90 long, 1.95 wide and 0.90 high at PLE. Ocular area 2.60 long, 3.00 wide anteriorly and
3.15 wide posteriorly. Diameter of AME 0.53. Clypeus not marked, chelicera length 2.30. Abdomen 2.85 long, 1.75 wide. Length of leg segments: I: $1.70+1.00+$ $1.35+1.10+0.60 ;$ II: $1.25+0.75+0.80+0.75+$ 0.48 ; III: $1.35+0.75+0.75+0.85+0.50$; IV: $1.50+$ $0.78+0.98+0.90+0.58$. Leg spination. Legs I and II: Fm d 1-1; the rest of segments are spineless. Legs III and IV: Fm d 1-1; Tb rt 0-1, v 2ap; Mt 3ap. Coloration. Carapace dark red-brown, with black around eyes. Sternum and labium dark brown, covered with white hairs. Maxilla orange, chelicerae red-brown. Abdomen dark brown, but dorsum with three transverse white stripes 

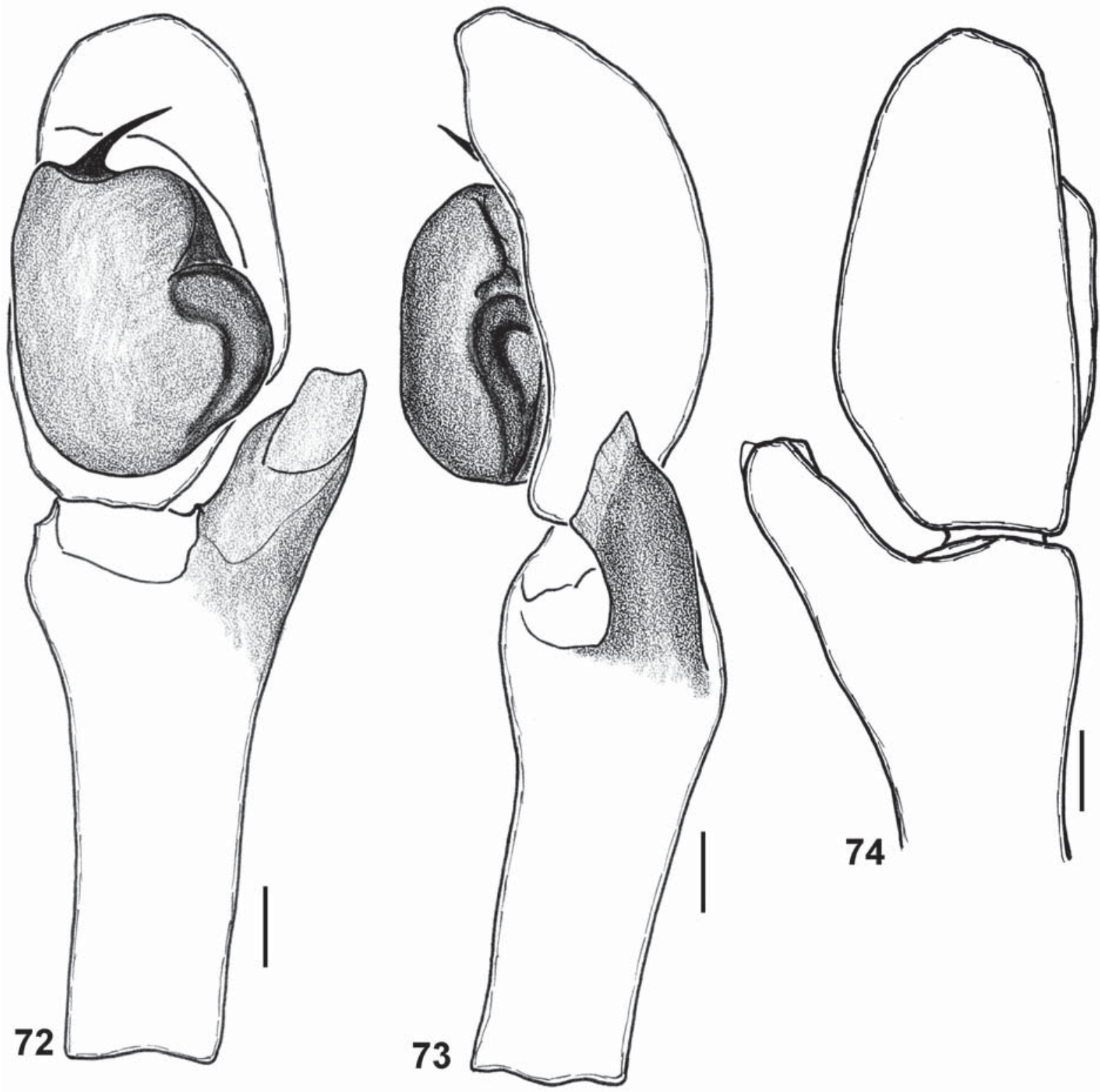

Figs 72-74. Male palp of Salticus ressli sp.n. (holotype): 72 — ventral view; 73 - retrolateral view; 74 - dorsal view. Scale bars: 0.1 $\mathrm{mm}$.

Рис. 72-74. Пальпа самца Salticus ressli sp.n. (голотип): 72 - вид снизу; 72 — вид сзади-латерально; 73 - вид сверху. Масштаб 0,1 мм.

of scales. Book-lung covers and spinnerets dark brown. All legs brown, with Mt and Tr of all legs visibly lighter (yellow-brown). Palps brown, their structure as in Figs 72-74; embolus stiletto-like, directed anteriolaterad, bulbus pear-shaped, tibial apophysis wide, with the 'excavated' tip.

FEMALE unknown.

\section{New faunistic records}

Aelurillus blandus (Simon, 1871)

MATERIAL. GREECE: 1 \% (SMFM), Karpaathos, Pigadia, no date, RK.
COMMENTS. Greece (mainland and Crete) only [Metzner, 1999; Azarkina, 2002; Dobroruka, 2002].

Aelurillus concolor Kulczyński, 1901

MATERIAL. TURKEY: 1 \% (NHMW), Erdschias area (Nside), nr. Lifos (c. $38^{\circ} 35^{\prime} 30^{\prime \prime} \mathrm{N}, 35^{\circ} 29^{\prime} \mathrm{E}$ ), 25-30.05.1902, A. Penther; $1 \mathrm{O}^{7}$ (NHMW), Burdur Gölü (label is illegible), 18.09.1955.

COMMENTS. Greece to Kyrgyzstan, south-eastward to Kerman Province of Iran [Azarkina, Mirshamsi, 2014; present data].

Aelurillus conveniens (O. Pickard-Cambridge, 1872).

MATERIAL. EGYPT: 1 (NHMW), Sinai, Gebel Musa (=Jebel Musa; c. $\left.28^{\circ} 32^{\prime} \mathrm{N}, 33^{\circ} 58^{\prime} \mathrm{E}\right), 10.04 .1982$, HEH. 
COMMENTS. Restricted to the Near East: Egypt, Israel and Syria [Prószyński, 2003].

\section{Aelurillus luctuosus (Lucas, 1846)}

MATERIAL. ALGERIA: $20^{7} \sigma^{7}$ (PCRB), Wilaya Blida, Atlas Blidéen, Chrea O., peak E. Abdelkader, c. 1520 m a.s.1., pitfall traps in Cedrus forest with Berberis bushes, 20.06.1987-9.05.1988, RB. - SPAIN: $2 O^{7} O^{7}$ (MVHN), Valencia, Alicante, Vall d'Ebo, Exterior Avenc Ample, 25.05.2005, SM; $1 \sigma^{7}$ (MVHN), Valencia,

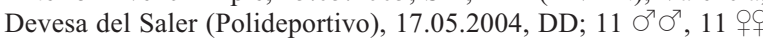
(NMPC), Xávia (=Jávea), NP del Mongú, 29.04.2004, LD; $20^{7} 0^{7}$, 1 ㅇ (NMPC), Valencia, Guadalest (c. $\left.38^{\circ} 41^{\prime} \mathrm{N}, 0^{\circ} 11^{\prime} \mathrm{E}\right), 27.04 .2004$, LD; $1 \mathrm{O}^{7}$ (MMUM; G7512.5347), Murcia, Sierra da Espuna, under stones above treeline, 19.05.1986, ED; 1 ○' (SMFM), Castellon Prov., W Atzeneta del Maestrat, Cova Obscura NW Eremita de Sant Joan $\left(40.20002^{\circ} \mathrm{N},-0.21203^{\circ} \mathrm{W}\right), 550 \mathrm{~m}$ a.s.l., free walking in deeper humid parts of cave, 30.05.2010, SH \& AS

COMMENTS. A Mediterranean species known from Portugal and Morocco, throughout Greece (incl. Crete) and Tunisia [Azarkina, Logunov, 2006], to Turkey [Danişman et al., 2012; Coşar et al., 2014].

Aelurillus v-insignitus (Clerck, 1758)

MATERIAL. TURKEY: $1 \sigma^{7}$ (SZMN), Artvýn Prov., Hopa, 17.05.1997, V. Bryja; 4 Ơ $^{7}$ (NHMW), Akșehir, Sültandag, 24.04 26.05.1960, FR; 1 O $^{7}$ (NHMW), Akşehir, 22.05.1960, Rasen; $1 \sigma^{7}$ (PCSD) Kazilibelli pass, S of Denizli, 29.05.1997, RS.

COMMENTS. A trans-Eurasian temperate species [Logunov, Marusik, 2000b].

Asianellus festivus (C.L. Koch, 1834)

MATERIAL. SPAIN: 1 + (MHNG), Barcelona, 12.1981, A. Senglet \& PH. - TURKEY: 1 (NMPC), nr. Pazar, c. $24 \mathrm{~km} \mathrm{~W}$ of Tokat $\left(40^{\circ} 16^{\prime} \mathrm{N}, 36^{\circ} 17^{\prime} \mathrm{E}\right)$, Ballica, nr. Mağarasi cave, 29.061.07.2002, MR. - RUSSIA: 1 \& (SZMN), Krasnodar Territory, nr. Adler, c. $6 \mathrm{~km}$ upstream of Mzynta river, 28.09.1993, O.V. Lyakhov.

COMMENTS. A trans-Eurasian temperate species, known from Spain [present data] to the Kuril Islands [Logunov, Marusik, 2000b]. New record for Spain.

Asianellus potanini (Schenkel, 1963)

MATERIAL. ARMENIA: $1 \sigma^{7}$ (ZISP, A.No. 202-1938), nr. Yerevan, 21.05.1938, A. Rikhter \& M. Ter-Minasyan.

COMMENTS. A Caucaso-Siberian subboreal species, known from Armenia [present data] to central China [Logunov, Marusik, 2000b]. New record for Armenia.

Ballus chalybeius (Walckenaer, 1802)

MATERIAL. ALGERIA: $1 O^{7}$ (PCRB), Wilaya Tizi Ouzou, Massif Djurdjura, Tala Gulief, c. $1500 \mathrm{~m}$ a.s.l., pitfall traps in Quercus plusieurs forest, 25.11.1986-29.05.1987, RB; 1 o (PCRB), Wilaya Boumerdes, Reghia, $45 \mathrm{~m}$ a.s.l., pitfall pitfall traps in degaraded Quercus suber forest, 13.06.1988, RB; 1 o (PCRB), same locality, Olea europea orchard, 3.05.1988, RB; 107 (PCRB), Wilaya Oran, forest nr. Msila, 400 m a.s.l., sieving Quer cus suber litter, among stones and Oxalis, 25.04.1984, RB; 2 \% (PCRB), Wilaya Tipasa, Bouchaoui, c. $95 \mathrm{~m}$ a.s.l., pitfall traps in Ulmus forest and Eucalypus plantation, 1.01.1987-12.01.1988, no date, RB; 1 + (PCRB), Wilaya Tipasa, Sidi Fredj, c. 10 m a.s.l., beating off branches in Pinus halepensis forest, 12.06.1987, RB; 1 o (PCRB), Wilaya Bejaia, S. Tichi, c. $50 \mathrm{~m}$ a.s.l., under shrubs along Wadi Djemaa, 20.05.1988, RB. - PORTUGAL: 4 우요 (FMNH), Mértola (c. $\left.37^{\circ} 38^{\prime} \mathrm{N},-7^{\circ} 40^{\prime} \mathrm{W}\right), 27.05 .2003$, PC. SPAIN: 1 o (MHNG), Zamora, nr. Sandin de Carballeda, 17.07. 1975, PH. - GREECE: 1 o (MHNG), Crete, Lassithi, the road Lasithi-Irakleon nr. junction to Krasi, c. 570 m a.s.1., 7.05.1985,
CL; $1 O^{7}$ (SMFM), NE hillside of Ossa Mts, above Omolion, $500 \mathrm{~m}$ a.s.1., 20.04.1968, JMS. — TURKEY: 1 \& (LMNM), İsparta, Kovada Gölü, sparsely vegetated lakeside shingle, 20.06.1993, SJ \& CF; 1 ( $(\mathrm{LMNM})$, c. $4 \mathrm{~km}$ E of Elicek, Corum $\left(40^{\circ} 18^{\prime} 38^{\prime \prime} \mathrm{N}, 34^{\circ} 42^{\prime} 51^{\prime \prime} \mathrm{E}\right)$, c. $1192 \mathrm{~m}$ a.s.l., willow-lined river bank below Quercus hillside, 6.07.1995, SJ \& CF; 2 오 (NMPC), nr. Pazar, c. 24 km W of Tokat $\left(40^{\circ} 16^{\prime} \mathrm{N}, 36^{\circ} 17^{\prime} \mathrm{E}\right)$, Ballica, nr. Mağarasi cave, 29.06-1.07.2002, MR; 1 + (ZMTU), Izmir Distr., Yamanlar Daği, litter of deciduous forest (Platanus-Rubus-Crataegus-Quercus-Pinus), 25.05.1973, PL; 2 우 (NHMW), Tekketal, SW Akşehir, 1100-1200 m a.s.l., pine forest with Quercus coccifera, Corylus, etc., 11.06.1967, JG, FR \& AR; 1 ( NHMW), c. $20 \mathrm{~km} \mathrm{NE}$ of Eskisehir, c. $1000 \mathrm{~m}$ a.s.l., oakpine forest, 13.06.1967, JG, FR \& AR; 2 우 (NHMW), Kizilcahaman, 1000-1200 m a.s.l., thicket of fruit trees, 27-28.05.1967, JG, FR \& AR; $10^{7}, 1$ ( $(\mathrm{NHMW})$, Akpehir, Sültanda ğ, 5.06.1960, FR; $1 \mathrm{O}^{\text {T }, 2} 2$ 우 (NHMW), Namrun, pine forest, 12.05-5.06.1964, FR; 1 ㅇ (NHMW), Namrun, S-slope of Taurus, $1200 \mathrm{~m}$ a.s.l., 911.05.1969, AR. - ABKHAZIA: $1 \sigma^{7}$ (ZMUM), nr. Sukhum, Canyon Esher, ferns and butcher's broom (Ruscus aculeatus), 8.06.2004, EM; $1 \mathrm{O}^{\text {T }}$ (ZMUM), nr. Sukhum, left bank of Kelasuri River (c. $3 \mathrm{~km}$ upstream of its mouth), alder thicket, 5.06.2004, EM. - GEORGIA: 1 ( (ZISP, A.No. 301-470), Kolkhida, N. Chkhugunetch, 500-650 m a.s.1., 3-10.06.1969, EP; 1 O$^{7}$ (SZMN), Kolkhida, Patara Poti, on the ground, 11.04.1988, DL; $2 \mathrm{O}^{7} \mathrm{O}^{7}$ (ZMUM), E of Poti, Chaladidi, alder-oak forest on swamp, in litter, 13.04.1983, SG. - RUSSIA: 2 우 (ZMUM), North Ossetia, c. 10 $\mathrm{km} \mathrm{N}$ of Mozdok, Acacia hedge, in litter, 28.05.1982, SG. AZERBAIJAN: 1 ( (ZMUM), Naxçivan, nr. Bichenek, $1900 \mathrm{~m}$ a.s.1., 13.07.1988, PD. - SYRIA: $1 \sigma^{7}, 1$ + (NMPC), NW Syria, Mashtal Helu nr. Homs, 30.03-3.04.2001, MR; $2 \mathrm{O}^{7} \mathrm{O}^{7}$ (NMPC), NW Syria, Slunfeh nr. Latakia, oak forest, 9.04.2001, MR.

COMMENTS. A European - Central Asian species, known from Portugal and Algeria [Helsdingen, 2014; present data], throughout the Mediterranean to Kyrgyzstan [Logunov, Rakov, 1998]. New records for Algeria and Syria.

\section{Ballus rufipes (Simon, 1868)}

MATERIAL. CYPRUS: $10^{7}$ (PCDM), house in Dali, in garden, spring 2004, DMC. - TURKEY: 1 (NMPC), Çağlayan, c. $24 \mathrm{~km} \mathrm{SE}$ of Erzincan $\left(39^{\circ} 36^{\prime} \mathrm{N} 39^{\circ} 42^{\prime} \mathrm{E}\right)$, valley above waterfall, 23-24.06.2002, MR.

COMMENTS. A Mediterranean species, recorded from the Iberian Peninsula to Turkey [Helsdingen, 2014; present data]. New records for Cyprus and Turkey.

\section{Bianor albobimaculatus (Lucas, 1846)}

MATERIAL. ALGERIA: $3 \sigma^{7} \sigma^{\top}, 6$ 우 (PCRB), Wilaya El Tarf, El Kala, $\mathrm{N}$ of Lac Tonga, c. $10 \mathrm{~m}$ a.s.1., pitfall traps in $P$. halepensis forest on dunes, 27.03.1988, RB; $1 O^{7}$ (PCRB), Wilaya El Tarf, El Kala, coast nr. Cap Rosa, El Wadi in Nhal, c. 2 m a.s.l., in litter of Ouercus suber and Chamaerops humilis along rivulet, 4.04.1982, RB. - SPAIN: $20^{7} \sigma^{7}, 4$ 우 (NMPC), Catalonia, Deltebre (c. $\left.40^{\circ} 44^{\prime} \mathrm{N}, 0^{\circ} 50^{\prime} \mathrm{W}\right), 26.04 .2004, \mathrm{LD} ; 1$ ( NMPC), Catalonia, Riumar, 26.04.2004, LD; $1 \mathrm{O}^{7}$ (NMPC), Catalonia, Mataró (c. $41^{\circ}$ $\left.32^{\prime} \mathrm{N}, 2^{\circ} 27^{\prime} \mathrm{E}\right), 1.05 .2004, \mathrm{LD} ; 1$ + (NHRS), Andalucia, Fuengirola, Rio Fuengirola, among vegetation in moist site, 19.05.1977, TK. — GREECE: 19 (SMFM), Crete, c. $8 \mathrm{~km} \mathrm{~W}$ of Chania, NW-shore of reservoir nr. Ajia, high grass with Umbellifera and Compositae, 23.04.1958, HK. - TURKEY: $10^{7}$ (LMNM), Içel, Silifke, c. $1 \mathrm{~km}$ E of Tasucu $\left(36^{\circ} 18^{\prime} 37^{\prime \prime} \mathrm{N}, 33^{\circ} 54^{\prime} 14^{\prime \prime} \mathrm{E}\right)$, canalised river marsh with Phragmitis, Juncus and Mentha, alongside timber yard, 6.05.1994, SJ \& CF. - SYRIA: 1 q (SMFM), Hama Prov., W slope of alGhab, karst spring, fish-ponds of Sattqa, 22.03.1979, RK. - AZERBAIJAN: $1 \mathrm{O}^{\top}$ (SZMN), Lenkoran Area, Vel' $\left(38^{\circ} 38^{\prime} \mathrm{N}, 48^{\circ} 52^{\prime} \mathrm{E}\right)$, 22.05.2003, EG; 1 O $^{7}$ (ZISP, A.No. 273-1909), same area, Damakh, 1.07.1909, A. Kirichenko.

COMMENTS. This species has been recorded under many names from South Africa to the Mediterranean, with Algeria and Portugal being the westernmost 
limits of its range, eastward to Central Asia [Logunov, 2001a, 2009a]. New record for Turkey.

Carrhotus xanthogramma (Latreille, 1819)

MATERIAL. SPAIN: $2 \sigma^{7} \sigma^{7}$ (NMPC), Besalú, 2.05.2004, LD - CROATIA: 1 (SMFM), Primorije-Gorski kotar, Rab Isl., hand collecting, 12-15.05.2008, JA \& JM. - SLOVENIA: 1 \& (SMFM), $\mathrm{S}$ of Komen $\left(45^{\circ} 48^{\prime} 20.61^{\prime \prime} \mathrm{N}, 13^{\circ} 45^{\prime} 52.56^{\prime \prime} \mathrm{E}\right)$, Karst, semi-natural meadow with stonewalls, bushes and trees, 27.06-5.07.2001, PJ. - TURKEY: 1 † (LMNM), Içel, Çamlıyayla, Dağ Hotel area $\left(37^{\circ} 10^{\prime} 10^{\prime \prime} \mathrm{N}, 34^{\circ} 36^{\prime} 02^{\prime \prime} \mathrm{E}\right)$, c. $1454 \mathrm{~m}$ a.s.l., limestone cliff, 1.07 1995, SJ \& CF. - RUSSIA: $10^{7}$ (SZMN), The Crimea, Bakhchisarai, 24-26.06.1993, SV; 1 O' (ZMUM), Daghestan, Kiziyurt Distr., Kakayurt river valley, 20.05.1981, O.V. Voltzit; 1 ( (ZMUM), Krasnodar Territory, Goryachiy Klyuch, c. $10 \mathrm{~km} \mathrm{~S}$ of Fanagoriyskoe Vil., mixed forest, 30.10.1981, SG; $10^{7}$ (ZISP, A.No 301), same territory, nr. Essentuki, 4.05.1955, Garnovskii; 1 9 (ZISP), Krasnodar Territory, nr. Khosta, 13-31.08.1929, coll.? GEORGIA: 1 ( (ZISP), Batumi, in grass, 14.08.1914, A. Bykov. - AZERBAIJAN: $10^{7}$ (ZMUM), Lenkoran Area, nr. Kirovsk (now Istisu), $200 \mathrm{~m}$ a.s.l., 8.05.1985, PD; 1 + (ZMUM), same area, Balyabir, 6.05.1985, PD; 1 O (ZISP), Shemakha Area, Pirkuli Reserve, 1000-1100 m a.s.l., 24.05.1984, DL.

COMMENTS. An amphi-Eurasian subboreal-subtropical species, known from Portugal to the S Urals, and then from Transbaikalia to Japan; the species is absent from west and central Siberia [Logunov, Marusik, 2000b].

\section{Chalcoscirtus infimus (Simon, 1868)}

MATERIAL. ALGERIA: 1 + (PCRB), Wilaya Blida, Meftah, Djebel Zerouela, c. $450 \mathrm{~m}$ a.s.l., among stones and Graminea in maquis, 23.05.1985, RB; 1 O' $^{\lambda}$ (PCRB), Wilaya Blida, Atlas Blidéen, Djebel Mouzaia, c. $1250 \mathrm{~m}$ a.s.l., under stones along the lake, 14.05.1988, RB; $1 \mathrm{O}^{7}$ (PCRB), Wilaya Blida, Atlas Blidéen, Chrea E., peak Fertasse, c. $1450 \mathrm{~m}$ a.s.l., pitfall traps in Cedrus forest, 12.04.1987-9.05.1988, RB; 1 O', 1 क (SZMN), Wilaya Blida, Chrea, c. $1550 \mathrm{~m}$ a.s.1., mountain grassland, 10.06.1985, RB; 1 9 (PCRB), Wilaya Aïn Defla, between Bordj Emir Khaled and Tarik Ibn Ziad, c. $700 \mathrm{~m}$ a.s.l., border of Wadi Massine, under stones and in litter, 18.05.1988, RB; 1 O $^{7}$ (PCRB), Wilaya Bejaia, E of Wadi Daas, c. $5 \mathrm{~m}$ a.s.l., under stones and on bushes on the shore, 22.05.1988, RB; 2 O $^{7} \sigma^{7}, 4$ 오 (ZMUM), Wilaya Bejaia, Tichi, c $10 \mathrm{~m}$ a.s.l., wasteland in dunes, in litter and beating off Acacia, 21.05.1988, RB; 1 + (PCRB), Wilaya Tipasa, Bouchaoui, c. $95 \mathrm{~m}$ a.s.1., pitfall traps in Pinus halepensis forest, 12.01-24.06.1988, RB; 1 O $^{7}$ (PCRB), Wilaya M'Sila, Chott el Hodna, S. Baniou, c. $400 \mathrm{~m}$ a.s.l., small dunes, in clumps of vegetation, 13.08.1988, RB; $1 \mathrm{O}^{7}$ (PCRB), Wilaya Boumerdes, Le Figuier, c. $5 \mathrm{~m}$ a.s.l., dunes nr. the beach, 2.05.1986, RB; $1 \mathrm{O}^{7}$ (PCRB), Wilaya Boumerdes, Zemmouri, $0 \mathrm{~m}$ a.s.1., litter on the shore, 31.05-7.07.1985, RB; 2 $\mathrm{O}^{7} \mathrm{O}^{7}$ (PCRB), Wilaya Tlemsen, Djebel Ourgla, NE of El Gor, c. $1290 \mathrm{~m}$ a.s.1., under stones and sieving litter in Quercus ilex forest, 5.05.1984, RB; $1 \mathrm{O}^{\mathrm{T}}$ (PCRB), Wilaya Oran, Daiet El Bragat along W18, c. $100 \mathrm{~m}$ a.s.l., among dry Salicornia and other herbs, 25.04.1984, RB; 1 ᄋ, 1 ( (ZMUM), Wilaya Tizi Ouzou, Wadi Youcef, c. $10 \mathrm{~m}$ a.s.l., pitrafll traps along wadi, 22.05.1988, RB; 1 $\mathrm{O}^{7}$ (PCRB), Wilaya Tizi Ouzou, forest Akfadou, Lake Agoulmin Aberkane, c. $1750 \mathrm{~m}$ a.s.l., among stones in Quercus faginea forest, 22.06.1984, RB; 1 ( (PCRB), Wilaya Bou Saada, Bou Saada, c. $560 \mathrm{~m}$ a.s.1., garden nr. hotel Transatlantique, 12.05.1988, RB. - PORTUGAL: $10^{7}$ (FMNH), Ribeira de Limas (c. $37^{\circ} 49^{\prime} \mathrm{N}$, $\left.-7^{\circ} 37^{\prime} \mathrm{W}\right)$, pitfall traps, 21.05.2003, PC; $3 \mathrm{O}^{7} \mathrm{O}^{7}$ (FMNH), Corredoura (c. $\left.37^{\circ} 45^{\prime} \mathrm{N},-7^{\circ} 39^{\prime} \mathrm{W}\right), 21.05-18.06 .2003$, PC; $1 \mathrm{O}^{\top}$ (FMNH), Mértola (c. $\left.37^{\circ} 38^{\prime} \mathrm{N},-7^{\circ} 40^{\prime} \mathrm{W}\right), 24.05 .2003, \mathrm{PC} ; 1 \mathrm{O}^{\prime \prime}, 1$ ㅇ (FMNH), Paúl do Boquilobo Biosphere Reserve (c. $39^{\circ} 23^{\prime} \mathrm{N},-8^{\circ} 32^{\prime} \mathrm{W}$ ), 28.055.07.2002, PC; $10^{7}$ (FMNH), Mira d'Aire (c. $\left.39^{\circ} 33^{\prime} \mathrm{N},-8^{\circ} 43^{\prime} \mathrm{W}\right)$, pitfall traps, 4.06.2002, PC. - SPAIN: $2 \sigma^{7} \sigma^{7}, 1$ ( 1 (NMPC), Xávia (=Jávea), NP del Mongú, 29.04.2004, LD. — GREECE: 1 Oフ $^{7} 1$ (LMNM), Lesbos, Vatera $\left(39^{\circ} 01^{\prime} 13^{\prime \prime} \mathrm{N}, 26^{\circ} 10^{\prime} 53^{\prime \prime} \mathrm{E}\right), 20.04 .1997$,
SJ; 1 오 (SMFM, 1986), Crete, Akrotiri plain, 05.1928, CR; 1 O (SMFM), Crete, SW Ajil deka, edge of Messara valley, sands with Medicago lupulina, 10.04.1958, HK; 1 O $^{7}$ (SMFM), Crete, valley NW of Lake Curna, rocky, dense bushes, Ficus, Quercus coccifera, on stones, 15.04.1958, HK; $1 \sigma^{7}$ (SMFM), Crete, nr. Ajil Deka, Gortys, E-slope, 85-100 m a.s.l., scree, bramble, Cirsium, few grass, 9.04.1958, HK; $1 \sigma^{7}$ (SMFM), Crete, W of Camaris, 600-800 m a.s.1., slope, rocky, Euphorbia, Liliacea, 6.04.1958, HK; 2 O $^{7} O^{7}$ (SMFM), N Crete, Gouves, 3.05.2008, HE \& UK; 2 $\mathrm{O}^{7} \mathrm{O}^{7}$ (SMFM), N Crete, Gouves, 2.05.2008, HE \& UK. - CYPRUS: $10^{7}$ (MHNG), nr. Tochni (between Larnaca and Limassol), under the bark of Carob tree, c. $100 \mathrm{~m}$ a.s.1., 24.03.1997, CL; 1 9 (PCMA), Gialia, 25.03.1998, MA. - TURKEY: 1 o (PCRS), Akyaka, on rocks behind Cinan Beach, 30.05.1996, ARS; $1 \sigma^{\top}, 2$ 우 (LMNM), Kayseri, c. $31 \mathrm{~km} \mathrm{~S}$ of Duvalli, rocky outcrop with Euphorbia and mixed scrub, 15.06.1993, SJ \& CF; 1 \& (LMNM), İsparta, nr. Uğurlu, 7.07.1992, SJ; $10^{7}$ (LMNM), Bağkonak, Sultan Dağlari, Konya, under dwarf hillside herbs, 19.09.1993, SJ \& CF; $1 \mathrm{O}^{\top}$ (LMNM), Kayseri, c. $1 \mathrm{~km}$ E of Dörtyal, Çol Gölü $\left(38^{\circ} 27^{\prime} 40^{\prime \prime} \mathrm{N}, 35^{\circ} 10^{\prime} 05^{\prime \prime} \mathrm{E}\right), 11.05 .1994, \mathrm{SJ} \& \mathrm{CF} ; 1 \mathrm{O}^{\prime 7}$ (LMNM), Niğde, c. $8 \mathrm{~km} \mathrm{~W}$ of Gözlükuyu, c. $1 \mathrm{~km}$ along Ilhara road off Askeray-Adana road, herb-rich, very rocky abandoned farmland edge, 10.05.1994, SJ \& CF; 2 O' $\sigma^{7}, 1$ ( (LMNM), Konya, Tuz Gölü (lake), Yavşan Tuslası ( $\left.38^{\circ} 45^{\prime} 43^{\prime \prime} \mathrm{N}, 33^{\circ} 09^{\prime} 31^{\prime \prime} \mathrm{E}\right)$, halophytic marsh, 9.05.1994, SJ \& CF; 1 क (NMPC), Sivas Prov., nr. Demiryurt, Tödürge Gölü (lake), c. $13 \mathrm{~km} \mathrm{~W}$ of Zara $\left(39^{\circ} 53^{\prime} \mathrm{N}, 37^{\circ} 37^{\prime} \mathrm{E}\right), 25-$ 28.06.2002, MR; 1 + (NMPC), nr. Pazar, c. $24 \mathrm{~km} \mathrm{~W}$ of Tokat $\left(40^{\circ} 16^{\prime} \mathrm{N}, 36^{\circ} 17^{\prime} \mathrm{E}\right)$, Ballica, nr. Mağarasi cave, 29.06-1.07.2002, MR; 1 (PCSD), nr. Dalyan, saltmarsh below Caunos, 18.05.1997, RS; 3 O $^{7} 0^{7}, 2$ 우 (PCSD), S of Denizli, Kanzikbelli pass, 29.05.1997, RS; $1 O^{\top}$ (PCLJ), Erdemli, under stone, 2.06.2004, LJ; 1 9 (NHMW), Hereke, c. $23 \mathrm{~km} \mathrm{~W}$ of Ižmit, 50-100 m a.s.l., olive plantation (under stones and in grass), 25.05.1967, JG. - SYRIA: $20^{7} 0^{7}$ (NMPC), S Syria, Qastaf Maaf, 13.04.2001, MR; 1 O $^{7}$ (NMPC), NW Syria, Mashtal Helu nr. Homs, 30.03-3.04.2001, MR.

COMMENTS. A Mediterranean - Central Asian subboreal species, known from the Azores and Iberian Peninsula [Wunderlich, 1992; Helsdingen, 2014], throughout the Mediterranean, Asia Minor and the Caucasus to Kyrgyzstan and Tajikistan [Logunov, Marusik, 1999; Marusik et al., 2004; Danişman et al., 2012; Coşar et al., 2014]. New record for Cyprus.

\section{Chalcoscirtus nigritus (Thorell, 1875)}

MATERIAL. TURKEY: $10^{\text {T }}$ (LMNM), İsparta, Kovada Gölü (lake), sparsely vegetated lakeside shingle, 20.06.1993, CF; $1 O^{7}$ (LMNM), İsparta, Eğridir Gölü (lake), vegetated shingle and lakeside sand, 20.06.1993, SJ \& CF; 1 O$^{\top}$ (LMNM), Kayseri, Demirkazýk, 12-13.06.1993, CF; 1 o , 1 o (NMPC), Sivas Prov., nr. Demiryurt, Tödürge Gölü (lake), c. $13 \mathrm{~km} \mathrm{~W}$ of Zara $\left(39^{\circ} 53^{\prime} \mathrm{N}\right.$, $\left.37^{\circ} 37^{\prime} \mathrm{E}\right), 25-28.06 .2002$, MR; 1 ( $(\mathrm{NMPC})$, nr. Pazar, c. $24 \mathrm{~km} \mathrm{~W}$ of Tokat $\left(40^{\circ} 16^{\prime} \mathrm{N}, 36^{\circ} 17^{\prime} \mathrm{E}\right)$, Ballica, nr. Mağarasi cave, 29.061.07.2002, MR; $1 \mathrm{O}^{\text {T }}$ (NHMW), Arapli gecidi, c. $50 \mathrm{~km} \mathrm{NE}$ of Niğde, c. $1400 \mathrm{~m}$ a.s.l., stony steppe, 8.06.1967, JG.

COMMENTS. A Euro-Siberian subboreal species, known from Germany in the west to NW China (Xinjiang) in the east [Logunov, Marusik, 2000b].

The recent record of C. tanasevichi Marusik, 1991 from Turkey [Danişman et al., 2012] may actually belong to $C$. nigritus and thus requires confirmation upon reference to the pertinent material. Both species are closely related and yet none of the Chalcoscirtus samples from Turkey examined by myself to date (see above) and by Coşar et al. [2014] belongs to $C$. tanasevichi. The westernmost locality of $C$. tanasevich $i$ known to date is Naxçivan (Azerbaijan) [Marusik et al., 2004]. 
Chalcoscirtus parvulus Marusik, 1991

Chalcoscirtus helverseni Metzner, 1999: (D $\odot$; holotype + from the Staatliches Museum für Naturkunde, Karlsruhe, Germany; examined). Syn.n.

For a complete set of taxonomic references see WSC [2015].

TYPE. Holotype $q$ of Chalcoscirtus helverseni (Staatliches Museum für Naturkunde) from Greece, Peloponnes Peninsula, Erimanthos-Tal, below Voidiaki, 9-12.06.1992, O.V. Helversen.

MATERIAL. GREECE: $1 \sigma^{7}$ (LMNM), Lesbos, Skala Polichaitos $\left(39^{\circ} 07^{\prime} 16^{\prime \prime} \mathrm{N}, 26^{\circ} 11^{\prime} 08^{\prime \prime} \mathrm{E}\right)$, Salicornia sward, Juncus and a variety of low-flowering herbs on salt pans, 20.04.1997, SJ. UZBEKISTAN: $1 \sigma^{7}, 5$ 우 (SZMN), c. $7 \mathrm{~km} \mathrm{~N}$ of Kitab, south foothills of Zeravshansky Mt. Range, c. 800 m a.s.1., 26.04.1993, DL \& A.A. Zyuzin. For more material studied see Logunov \& Marusik [1999].

COMMENTS. An eastern Mediterranean - Central Asian subboreal species, known from Greece [Metzner, 1999: sub. C. helverseni; present data] throughout Asia Minor to Tajikistan [Logunov, Marusik, 1999].

As was earlier suggested by Logunov [2001c], $C$. helverseni described from Greece from a single female could be a junior synonym of $C$. parvulus. A re-examination of the $q$ holotype of $C$. helverseni has proved this to be true. The holotype has got the identical epigyne and spermathecae to those of C. parvulus [Logunov, Marusik, 1999: figs 89-93]. The only difference between the $q$ holotype of $C$. helverseni and the o+ from Central Asia is the absence of two short white stripes at the rear end of the dorsum (usually present in Central Asian specimens; see Logunov \& Marusik [1999: fig. 91]). Such difference is likely to only reflect an intraspecific variation in body coloration. Thus, it is safe to conclude that the name $C$. helverseni is to be considered a junior synonym of $C$. parvulus.

\section{Chinattus caucasicus Logunov, 1999}

MATERIAL. AZERBAIJAN: 1 (SZMN), Astara Distr., between Motlayatat and Bilyasar, Talysh Mts $\left(38^{\circ} 36^{\prime} \mathrm{N}, 48^{\circ} 40^{\prime} \mathrm{E}\right)$ 600-700 m a.s.1., Carpinus-Acer-Quercus forest, 20-22.06.2003, AC.

COMMENTS. Restricted to the E Caucasus and NW Iran [Logunov, 1999; Logunov et al., 2002].

Cyrba algerina (Lucas, 1846)

MATERIAL. MOROCCO: 1 + (MHNG), region of Middle Atlas, Taza, in the 'wild garden', Hotel Friouato, c. $460 \mathrm{~m}$ a.s.l., 21.06.1990, BH. - ALGERIA: 1 O $^{7}$ (PCRB), Wilaya Bejaia, E of the mouth of Wadi Daas, c. 5 m a.s.l., under stones and on bushes on the shore, 22.05.1988, RB; 1 Or $^{7}$ (PCRB), Wilaya Blida, Atlas Blidéen, Djebel Mouzaia, c. $1250 \mathrm{~m}$ a.s.1., under stones along the lake, 14.05.1988, RB; 1 O (PCRB), Wilaya Bejaia, Tichi, c. $10 \mathrm{~m}$ a.s.l., wasteland in dunes, 21.05.1988, RB; 1 P (PCRB), Wilaya Ain Defla, between Bordj Emir Khaled and Tarik Ibn Ziad, c. 700 $\mathrm{m}$ a.s.l., border of Wadi Massine, under stones and in litter, 18.05 . 1988, RB; $10^{7}$ (PCRB), Wilaya Blida, Meftah, Djebel Zerouela, c. $450 \mathrm{~m}$ a.s.l., among stones and Graminea in maquis, 23.05.1985, RB; $2 O^{\top} O^{\top}$ (PCRB), Wilaya Oran, Daiet El Bragat along W18, c. $100 \mathrm{~m}$ a.s.l., among dry Salicornia and other herbs, 25.04.1984, RB. - PORTUGAL: $120^{7} 0^{\top}, 9$ 우 (FMNH), Mértola (c. $37^{\circ} 38^{\prime} \mathrm{N}$, $\left.-7^{\circ} 40^{\prime} \mathrm{W}\right), 24.05 .2003, \mathrm{PC} ; 2 \mathrm{O}^{7} \mathrm{O}^{7}, 2$ 우 $(\mathrm{FMNH})$, Ribeira de Limas (c. $37^{\circ} 49^{\prime} \mathrm{N},-7^{\circ} 37^{\prime} \mathrm{W}$ ), pitfall traps, 21.05.2003, PC; $10^{7}, 2$ 우 (FMNH), Paúl do Boquilobo Biosphere Reserve (c. $39^{\circ} 23^{\prime} \mathrm{N}$ $\left.-8^{\circ} 32^{\prime} \mathrm{W}\right)$, pitfall traps, $16-30.07 .2002, \mathrm{PC} ; 2 \mathrm{O}^{\top} \mathrm{O}^{\top}$ (FMNH), Planalto de St António (c. $39^{\circ} 30^{\prime} \mathrm{N},-8^{\circ} 42^{\prime} \mathrm{W}$ ), pitfall traps, 4.06 .2002 PC. - SPAIN: 1 + (MHNG), the Balearic Islands, Mallorca, Val
Ratjada, 11.04.1968, BH; 1 오 (MHNG), the Canaries, La Palma (SW part), between Ermita of Santa Cecilia and El Charco, nr. the road, Euphorbiaceae vegetation, various bushes and few Pinus canariensis, under rocks, c. $750 \mathrm{~m}$ a.s.1., 27.05.2000, CL; 2 O $^{7} \mathrm{O}^{7}$ (PCJK), Tenerife, Montagna Las Colorados, S of Villaflor, 25.04. 2004, HDK; 1 + (PCJK), Tenerife, Lorno Oliva, 1.05.2004, HDK; $10^{7}$ (PCJK), Tenerife, Santiago Del Teide, 26.04.2004, HDK; 6 $0^{\top} O^{7}, 4$ 우 (NMPC), Valensia, Guadalest (c. $38^{\circ} 41^{\prime} \mathrm{N}, 0^{\circ} 11^{\prime} \mathrm{E}$ ), 27.04.2004, LD; $10 \sigma^{7} \sigma^{7}, 5$ 우 (NMPC), Xávia (=Jávea), NP del Mongú, 29.04.2004, LD; 1 O , 4 우 (NMPC), Calp, 29.04.2004, LD; 2 +o (SMFM), Murcia Prov., W of Alhama de Murcia, Sierra de Espuna $\left(37.85601^{\circ} \mathrm{N}, 1.49431^{\circ} \mathrm{W}\right)$, c. $691 \mathrm{~m}$ a.s.1., Pinus forest and open pastures, under dry stones, 4.06.2010, SH \& AS; $10^{77}, 1$ 和 (SMFM), Castellon Prov., W Atzeneta del Maestrat, Cova Obscura NW Eremita de Sant Joan $\left(40.20002^{\circ} \mathrm{N}, 0.21203^{\circ} \mathrm{W}\right), 550 \mathrm{~m}$ a.s.1., free walking in deeper humid parts of cave, 30.05 .2010 , SH \& AS; $20^{7} O^{7}, 1$ + (SMFM), Castellon Prov., E Ain, Cova del Eremita (39. $\left.90180^{\circ} \mathrm{N}, 0.33677^{\circ} \mathrm{W}\right), 460 \mathrm{~m}$ a.s.l., at cave entrance in nearby stone ruin, 31.05.2010, SH \& AS. — LIBYA: 2 ㅇ (SMFM, 4150), Tripoli, Vytina, no date and collector name. - GREECE: 1 \% (SMFM), S coast of Peloponnes, Foinikous, lime and sanf cliffs at seashore, 4.06.1998, LM; 2 O $^{7}, 2$ 우 (SMFM), S coast of Peloponnes, Ligia, beach, dunes and adjacent cultural landscape, 1.06.1998, LM; 1 \& (SMFM), Island Kos, nr. Zia, 500-800 m a.s.1., forest and macchia, 29.04.1968, JMS; $1 \sigma^{7}, 4$ 우 (SMFM, 4151), Crete, Akrotiri plain, 05.1928, CR; $1 \mathrm{O}^{7}$ (SMFM), N Crete, Gouves, 3.05.2008, HE \& UK; $1 \mathrm{O}^{\top}$ (MHNG), Island of Rhodes, Mt. Profitis Ilias, c. $650 \mathrm{~m}$ a.s.1., 15.05.2001, CL; $1 \mathrm{O}^{\text {T }}$ (MHNG), Island of Rhodes, above Arnitha (S part of the isle), c. $200 \mathrm{~m}$ a.s.1., 16.05. 2001, CL. - CYPRUS: $1 \sigma^{7}$ (MHNG), Protaras, Ayios Elias Vil., 2.04.1995, PH. - TURKEY: $10^{7}, 1$ ( (PCRS), Akyaka, Sulemin's castle, under stones, 31.05.1996, ARS; 3 우 (PCRS), Mazi Beach, nr. Bodrum, under stone in olive grove, 7.06.1997, ARS; 1 o (LMNM), Adana $\left(36^{\circ} 59^{\prime} \mathrm{N}, 35^{\circ} 21^{\prime} \mathrm{E}\right)$, Rasit Ener Motel, light trap, 27.06.1995, SJ \& CF; 1 o (LMNM), Icel, Narlıkuyu (36 $26^{\prime} 04^{\prime \prime} \mathrm{N}$, $34^{\circ} 06^{\prime} 44^{\prime \prime} \mathrm{E}$ ), garrigue vegetated limestone dominated by Pinus, 5.06.1994, MB; $1 \mathrm{O}^{7}, 1$ क (LMNM), Niğde, c. $8 \mathrm{~km} \mathrm{~W}$ of Gözlükuyu, c. $1 \mathrm{~km}$ along Ilhara road off Askeray-Adana road, herbrich, very rocky abandoned farmland edge, 10.05.1994, SJ \& CF; 1 $\sigma^{7}$ (LMNM), Zonguldak, Amasra, waste ground by flats, 18.05.1994, SJ \& CF; 1 ㅇ (NMPC), Camlik, nr. Seydisehir, pastures on limestone, 12.07.2003, MR; 2 우 (NMPC), Tunceli (39 $\left.06^{\prime} \mathrm{N}, 39^{\circ} 33^{\prime} \mathrm{E}\right)$,

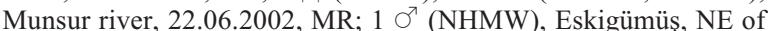
Niğde, 1400-1500 m a.s.1., steppe on the edge of orchard, 9.06.1967, JG, FR \& AR; $1 O^{7}$ (NHMW), Hisarc1k, SE Kayseri, 1500-1600 m a.s.l., sparce oak forest, 7.06.1967, JG, FR \& AR; $2 \mathrm{O}^{7} \mathrm{O}^{7}$ (NHMW), Güvem, under stones, 1962-1964, FR; 1 (NHMW), Talas, 7.06. 1965, FR; 3 O $^{7} 0^{7}$ (NHMW), Finike/Grolle, 3.05.1964, FR. TUNISIA: 1 ( $(M H N G)$, c. $13 \mathrm{~km}$ of Zarzis (S de la Tunisie), nr.

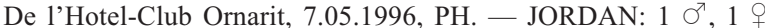
(SMFM), Dana Nature Reserve, Wadi Dana $\left(30^{\circ} 41^{\prime} \mathrm{N}, 35^{\circ} 37^{\prime} \mathrm{E}\right)$, under stones, 16.04.2004, JA \& JM; 1 ( (SMFM), Ma'an Prov., NW of Ma'an, Wadi Musa, Petra [Batra'] Valley, 17.03.1977, RK. - AZERBAIJAN: 1 o (ZISP), Lenkoran Area, nr. Alexeevka, 23.06.1983, DL; 4 오 (SZMN), same area, Hyrkan Reserve, under tops of beehives, 21.06.1983, DL; 4 Oొ $^{7}$ (ZMUM), c. $60 \mathrm{~km} \mathrm{SW}$ of Baku, nr. Gobustan, Gobustan Reserve, 7.05.1989, PD; 1 ㅇ (ZMUM), nr. Vartashen, $300 \mathrm{~m}$ a.s.1., 7.07.1978, PD; 1 + (SZMN), Astara Distr., between Motlayatat and Bilyasar, Talysh Mts $\left(38^{\circ}\right.$ $\left.36^{\prime} \mathrm{N}, 48^{\circ} 40^{\prime} \mathrm{E}\right), 600-700 \mathrm{~m}$ a.s.1., Carpinus-Acer-Quercus forest, 20-22.06.2003, AC.

COMMENTS. A Mediterranean - Central Asian subboreal-subtropical species known from the Mediterranean, including the Canary Islands and north Africa, throughout the Near East [e.g., Uyar, Uğurtaş, 2012; Coşar et al., 2014], to India and Tajikistan [Logunov, Rakov, 1998; Logunov, 2013]. New records for Morocco, Libya and Jordan.

Euophrys frontalis (Walckenaer, 1802)

MATERIAL. SPAIN: $20^{7} 0^{7}, 1$ (NMPC), Catalonia, Deltebre (c. $\left.40^{\circ} 44^{\prime} \mathrm{N}, 0^{\circ} 50^{\prime} \mathrm{W}\right), 26.04 .2004$, LD. - GREECE: $10^{\top \prime}$ (SMFM), 
Chalkidiki island, Andrea, c. $800 \mathrm{~m}$ a.s.1., forest, 1968, JMS; $1 \sigma^{7}$ (SMFM), Dugo Selo, mixed forest, 14.05.1968, JMS. - TURKEY: $1 \sigma^{\top}$ (LMNM), Kastamonu, c. $3 \mathrm{~km} \mathrm{~N}$ of Sakiz $\left(41^{\circ} 40^{\prime} 06^{\prime \prime} \mathrm{N}\right.$, $\left.34^{\circ} 39^{\prime} 13^{\prime \prime} \mathrm{E}\right), 1111 \mathrm{~m}$ a.s.1., edge of road into conifer plantation over

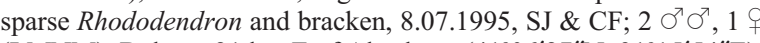
(LMNM), Bolu, c. $21 \mathrm{~km} \mathrm{E}$ of Akçakoca $\left(41^{\circ} 06^{\prime} 27^{\prime \prime} \mathrm{N}, 31^{\circ} 15^{\prime} 54^{\prime \prime} \mathrm{E}\right)$, sparse mixed weeds on road slope above snore, wet field with Juncus adjacent to stream, 18.05.1994, SJ \& CF; 1 フ 4 우 (NMPC), Tunceli $\left(39^{\circ} 06^{\prime} \mathrm{N}, 39^{\circ} 33^{\prime} \mathrm{E}\right)$, Munsur river, 22.06.2002, MR; $1 \sigma^{\prime}$ (NHMW), Tekketal, SW Akşehir, 1100-1200 m a.s.l., pine forest with Quercus coccifera, Corylus, etc., 11.06.1967, JG, FR \& AR; $\sigma^{7}$ (NHMW), c. $20 \mathrm{~km} \mathrm{~N}$ of Turhal, $800-1000 \mathrm{~m}$ a.s.l., pine forest 4.06.1967, JG, FR \& AR; $1 \sigma^{7}, 1$ ( NHMW), c. 5 km NE of Ulubey, c. $350 \mathrm{~m}$ a.s.1., decidous forest of Castanea, Quercus, Robinia, Carpinus, etc., 2.06.1967, JG, FR \& AR; $1 \sigma^{7}$ (NHMW), c. 8 km S of Tokat, c. $900 \mathrm{~m}$ a.s.1., oak forest with Carpinus, Pinus, etc., 5.06 1967, JG, FR \& AR; 1 ○ (NHMW), Kizilcahaman, 1000-1200 m a.s.1., thicket of fruit trees, 27-28.05.1967, JG. - ABKHAZIA: 1 ○ (ZMUM; black form), Novyi Afon, valley of river Psyrtskhe, Carpinus forest, 6.06.2004, EM; 1 ○ (ZMUM), nr. Sukhum, left bank of Kelasuri River (2-3 km upstream of its mouth), 5.06.2004, EM. AZERBAIJAN: $2 \sigma^{7} \sigma^{7}$ (dark form) (SZMN), Lenkoran Area, Lenkoran Reserve $\left(38^{\circ} 38^{\prime} \mathrm{N}, 48^{\circ} 47^{\prime} \mathrm{E}\right), 23.05 .2003, \mathrm{EG} ; 2$ oᄋ $(\mathrm{SZMN}), \mathrm{nr}$ Lenkoran, in rush, 17-19.07.1983, DL; 1 ア, 2 우 (dark form) (SZMN), same area, nr. Alexeevka, rush, 6.07.1983, DL; 1 (ZMUM; earlier identified by PD as Talavera petrensis), Lerik Distr., nr. Gosmalyan, 1400 m a.s.1., 19.05.1985, PD; 3 oO (ZISP), Karabakh, Gadrum, nr. Azokh, 8.08.1986, PD; 19 (ZISP), Kakh Distr., nr. Ilisu, 11.07.1989, S. Dashdamirov; 1 \% (SZMN), Ismailly Distr., Ismailly Reserve, nr. Buinus Vil., 700 m a.s.1., 25-26.06.2003, AC.

COMMENTS. A trans-Eurasian temperate species [Logunov, Marusik, 2000b] that exists in two colour morphs, yellow (typical) and black ones (see Logunov [1997] for further details).

Euophrys rufibarbis (Simon, 1868)

MATERIAL. ITALY: 3 O $^{7} \mathrm{O}^{7}$ (MMUM), Sicily, R.N.O. Zingaro, nr. Trapani, pitfall traps, 17-31.09.2001, F. di Franco. CYPRUS: $1 \sigma^{7}$ (LMNM), Tróodos, nr. Kannoures, Juncus/grass, 9.09.1997, SJ \& CF

COMMENTS. This species was identified on the basis of the work by Metzner [1999]. However, the taxonomic status and distribution of E. rufibarbis is yet poorly understood. It seems that the species has a westMediterranean distribution, so far being recorded from the Iberian Peninsula, Corsica, Italy (both mainland and islands) to Greece (both mainland and islands), Macedonia and Croatia [Helsdingen, 2014]. The recent records of this species from Turkey [Coşar et al., 2014] were based on the singular females and need confirmation by the samples containing males. The records of this species from China [Song et al., 1999] were likely to be based on misidentifications. New record for Cyprus.

\section{Euophrys sulphurea (L. Koch, 1867)}

MATERIAL. GREECE: $10^{7}$ (LMNM), Lesbos, Angliki Skala, nr. Krioneri $\left(39^{\circ} 11^{\prime} 35^{\prime \prime} \mathrm{N}, 26^{\circ} 18^{\prime} 33^{\prime \prime} \mathrm{E}\right)$, pine woodland over mixed scrub alongside river, 24.04.1997, SJ; $1 \sigma^{7}$ (LMNM), Lesbos, Angliki Skala, nr. Krioneri $\left(39^{\circ} 11^{\prime} 35^{\prime \prime} \mathrm{N}, 26^{\circ} 18^{\prime} 33^{\prime \prime} \mathrm{E}\right)$, pine woodland over mixed scrub alongside river, 24.04.1997, SJ; $2 \sigma^{7} \sigma^{7}$ (LMNM), Lesbos, Vatera $\left(39^{\circ} 01^{\prime} 13^{\prime \prime} \mathrm{N}, 26^{\circ} 10^{\prime} 53^{\prime \prime} \mathrm{E}\right), 20.04 .1997$, CF \& SJ; $10^{7}$ (LMNM), Lesbos, Skala Kallonis $\left(39^{\circ} 12^{\prime} 25^{\prime \prime} \mathrm{N}, 26^{\circ}\right.$ $13^{\prime} 13^{\prime \prime} \mathrm{E}$ ), back of beach and along river, 23.04.1997, SJ; $10^{7}$ (SMFM), Crete, W of Camaris, S-slope, rocky, Phrygana, Liliacea, 6.04.1958, HK; $1 \sigma^{7}$ (NHRS), Chania Pref., at Amiros river E of Vrissees, on church wall, 15.05.2001, TK; $1 \sigma^{7}$ (NHRS), same pref., Georgioupoli, grassy area close to Almiros river, west side of the bridge, 6.05.2001, TK; $1 \sigma^{7}$ (NHRS), same locality, grass and Phragmites W of harbour, 14.05.2001, TK; $1 \mathrm{O}^{7}$ (NHRS), same locality, garrigue on hill slope W of Georgioupoli, 18.05.2001, TK; $2 \sigma^{7} \sigma^{7}$ (NHRS), same pref., at Lakki-Omalos Rd., pass entering Omalos plateau, phrygan (under stones), 11.05.2001, TK; $1 \sigma^{7}$ (PCJK), Crete, Elafronisos, 6.04.2002, J. Van Keer. - TURKEY: $1 \mathrm{O}^{\mathrm{T}}$ (PCRS), west part of the country, Thomsan Villas, Akyaka, on walls of house, 1.06.1996, ARS; 1 (LMNM), Konya, c. $10 \mathrm{~km}$ along Akșehir-Cetince road, Sultan Dağları, mixed herbs and shrubs among young pine plantation, 19.06.1993, SJ \& CF.

COMMENTS. A Mediterranean species, known from Portugal to Turkey and Syria [Helsdingen, 2014; present data]. However, there are two recognizable colour morphs of this species, of which one differs from the other in having the half-brown palps and completely brown chelicerae in males (entirely yellow in the males of the second form) and different proportions of the spermathecae. As it is known that the male colour characters in Euophrys (s.str.) are reliable in diagnosing closely related species [e.g., Logunov et al., 1993; Logunov, 1997], it is likely that at least two species are currently recorded under the name of $E$. sulphurea from the Mediterranean; see also comments under this species name in Logunov \& Chatzaki [2003]. The matter requires special attention in the future.

Euophrys terrestris (Simon, 1876)

MATERIAL. SPAIN: 2 oᄋ (NMPC), Catalonia, Mataró (c. $\left.41^{\circ} 32^{\prime} \mathrm{N}, 2^{\circ} 27^{\prime} \mathrm{E}\right), 1.05 .2004, \mathrm{LD}$

COMMENTS. Reported from the Iberian Peninsula, France (mainland and Corsica), Greece (Crete), Italy and Switzerland [Helsdingen, 2014; present data]. New record for Spain.

Evarcha arcuata (Clerck, 1758)

MATERIAL. GREECE: $1 \sigma^{7}$ (PCRG), Thasos, Thasos shell valley fen $\left(40^{\circ} 39^{\prime} \mathrm{N}, 24^{\circ} 40^{\prime} \mathrm{E}\right), 25 \mathrm{~m}$ a.s.1., 26.05.2003, $\mathrm{AF} ; 1$ 웅 (PCRG), same locality, Thasos shell pastures, 27.09.2001, coll.?; 1 o (SMFM), Kavala, Rentina Valley, 19.08.1971, RK. - CROATIA: 1 OT $^{7}$ SMFM), c. $30 \mathrm{~km} \mathrm{~S}$ of Sisak, nr. Muzilovcica, meadows nr. residual puddles, 20.04.2003, JA \& JM. — SLOVENIA: $10^{7}, 1$ ㅇ (SMFM), Slioica, Mt nr. Zirknitz, 800-1000 m a.s.1., 17.07.1974, JMS. - TURKEY: 2 O $^{7} 0^{7}, 1$ ㅇ (PCRS), c. $6 \mathrm{~km} \mathrm{~W}$ of Koycegiz, wet area in Platanus woodland, 5.06.1996, ARS; 1 ( (LMNM), c. $4 \mathrm{~km} \mathrm{~N}$ of Kambia, flowering herb, 14.09.1996, SJ \& CF; $1 \mathrm{O}^{7}$ (PCSD), Dalyan, c. $1 \mathrm{~km} \mathrm{~N}$ of Hotel Gol, 20.05.1997, RS; $1 \mathrm{O}^{7}$ (NHMW), c. $15 \mathrm{~km} \mathrm{NE}$ of Abantsee, Abant Mts, 900-1200 m a.s.1. Pinus silvestris forest, 26.05.1967, JG, FR \& AR; 1 O (BMNH), Trabzon, Hamsiköy, c. $1250 \mathrm{~m}$ a.s.l., beaten from low bushherbs on open hillside, 25.08.1958, coll.? - ABKHAZIA: 1 $\sigma^{7}$ (ZMUM), Sukhum, orchards, 7-11.06.2004, EM; 1 + (ZMUM), nr. Sukhum, left bank of Kelasuri River (c. $3 \mathrm{~km}$ upstream of its mouth), alder thicket, 5.06.2004, EM. - RUSSIA: 2 우 (SZMN), The Crimea, Bakhchisarai, 24-26.06.1993, SV; 1 + (SZMN), Krasnodar Territory, nr. Adler, c. $6 \mathrm{~km}$ upstream of Mzynta river, 28.09.1993, O.V. Lyakhov; 3 우으 (ZISP), Adygea, nr. Maikop, 9.06.1976, V.I. Ovtsharenko; 2 O $^{\top} 0^{\top}, 2$ 우 (ZISP), Adygea, Maikop Distr., Caucasian Reserve, Guzeripl' Mt., 650 m a.s.1., 1218.07.1984, V.I. Ovtsharenko; 1 ㅇ (ZISP, A.No. 285), Adygea Republic, offset of Mt. Abago, subalpine meadow on the slope of Kholodnaya balka, 2.06.1911, D. Filatov; 1 ㅇ (ZMUM), North Ossetia, Alagir Distr., betweeen Zinkar and Shubino, 21-23.06. 1981, S.K. Alexeev; $10^{7}, 3$ 우 (ZMUM), Karachaevo-Cherkessia, Teberdinsky Reserve, Teberda river valley, $1300 \mathrm{~m}$ a.s.l., swamp, 24.05.1986, KM; $2 O^{7} \sigma^{7}, 1$, 1 (ZISP), same republic, Indysh river, 4-8.08.1935, A. Dyakonov; $3 \sigma^{7} \sigma^{7}$ (ZISP, A.No. 179), Daghestan, Kizlyar, 9-10.05.1925, Kirichenko; 1 ㅇ (ZISP, A.No. 359), Stavropol Territory, Essentuki, 27.07.1914, A. Bartenev; $10^{7}$ (ZISP, 

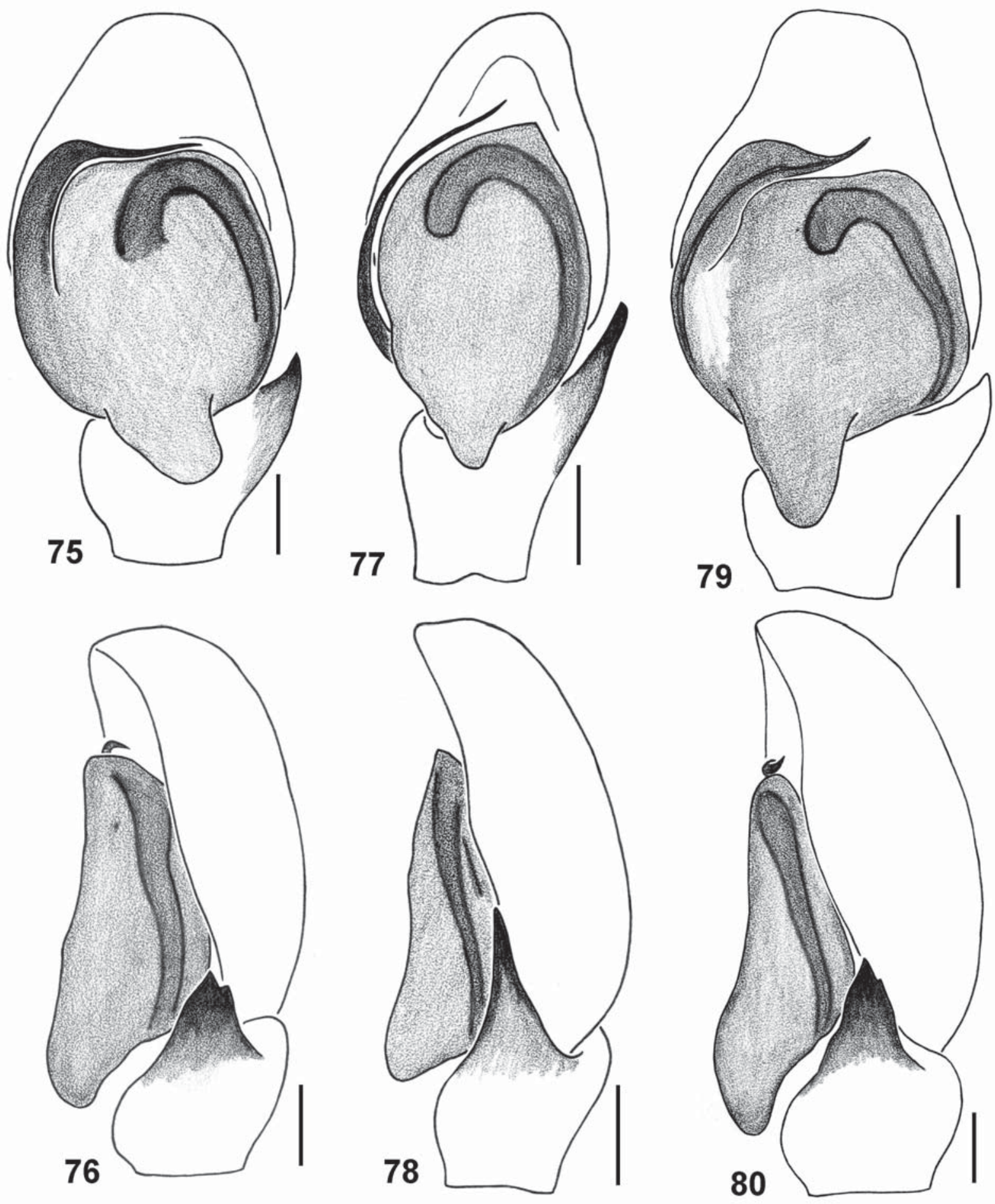

Figs 75-80. Male palps of Evarcha jucunda (Lukas, 1846) (75-76; Italy), E. patagiata (O. Pickard-Cambridge, 1872) (77-78; Cyprus) and E. eriki Wunderlich, 1987 (79-80; holotype): 75, 77, 79 - ventral view; 76, 78, 80 - retrolateral view. Scale bars: $0.1 \mathrm{~mm}$. Рис. 75-80. Пальпы самцов Evarcha jucunda (Lukas, 1846) (75-76; Италия), E. patagiata (O. Pickard-Cambridge, 1872) (77-78; Кипр) и E. eriki Wunderlich, 1987 (79-80; голотип): 75, 77, 79 - вид снизу; 76, 78, 80 - вид сзади-латерально. Масштаб 0,1 мм. 
A.No. 204), North Caucasus, nr. Saprat (uncertain locality), 7.08.1937, AD. - GEORGIA: 1 (ZISP), Adzharia, Tsoniarisi, $400 \mathrm{~m}$ a.s.1., sweeping, 18.06.1969, EP; $2 \sigma^{7} \sigma^{7}$ (ZISP), Lagodekhi Reserve, 7.07.1988, Yu. M. Marusik; 1 ○ (SZMN), Batumi, 10.08.1981, DL; $1 \sigma^{7}$ (ZISP), same locality, 14.08.1914, A. Bykov; $1 \sigma^{\top}$ (ZISP, A.No. 301-470), Kolkhida, N. Chkhugunetch, 500$650 \mathrm{~m}$ a.s.1., 3-10.06.1969, EP; $1 \sigma^{7}$ (ZISP), Batumi, sweeping, 27.06.(no year), coll.?; $5 \sigma^{7} \sigma^{7}$ (ZISP), nr. Bakuriani, 27.07.1917, N. Bartenev. - ARMENIA: $1 \sigma^{\top}$ (ZISP, A.No. 510-1931), [Tavush Distr.], nr. Dilizhan, 4.08.1931, EP. - AZERBAIJAN: $1 \sigma^{7}$ (ZISP), Lenkoran Area, nr. Kirovsk (now Istisu), 24.06.1985, coll.?; 1 O (ZISP), Lenkoran Area, nr. Alexeevka, in rush, 7.07.1983, DL; 1 O (ZISP), same area, nr. AzFilial, in rush, 2.07.1983, DL; 1 ○ (ZMUM), nr. Khachmas, 22.04.1986, PD; 1 (ZMUM), Kakhsky Distr., Vandam, 17.06.1986, PD; 1 ○े (SZMN), Shemakha Distr., Pirkuli Reserve, 15.09.1984, 1100 m a.s.1., DL.

COMMENTS. A trans-Eurasian temperate species [Logunov, Marusik, 2000b; Helsdingen, 2014].

\section{Evarcha armeniaca Logunov, 1998}

MATERIAL. TURKEY: $1 \sigma^{7}$ (PCRS), Mazi Beach, nr. Bodrum, under stone in olive grove, 7.06.1997, ARS. - AZERBAIJAN: 1 (MMUM), Gobustan Reserve, Beyuk-Dash Mt $\left(40^{\circ} 07^{\prime} \mathrm{N}, 4^{\circ} 23^{\prime} \mathrm{E}\right), 17.05 .2003$, EG.

COMMENTS. Recorded from a few localities in Armenia, Azerbaijan and Turkey only [Logunov, 1998a; Logunov, Guseinov, 2002; present data]. New record for Turkey.

\section{Evarcha eriki Wunderlich, 1987}

Figs 79-80, 85-86, 91-92.

Evarcha eriki Wunderlich, 1987: 270, figs 682-684 (D ${ }^{7}+$ holotype $\sigma^{7}$ from Naturmuseum und Forschungsinstitutt Senckenberg, Frankfurt am Main, Germany; examined).

TYPES. Holotype or (SMFM, 37342) from Spain, the Canaries, Gran Canaria, c. 2 km S of Fataga, VII, J. Wunderlich. Paratype: SPAIN: $1+$ (SMFM, 37306), the Canaries, Gran Canaria, Bco. de Arguineguin, nr. Soria, VII, J. Wunderlich.

MATERIAL. SPAIN: 1 ㅇ (OUMNH), The Canaries, Gran Canaria, Chaveo of Maspalomas, beaten palm for sedum, 31.07.1957, GL

COMMENTS. The Canary Islands only [Wunderlich, 1987; present data].

See also below under the 'Comments' of E. jucunda.

\section{Evarcha falcata (Clerck, 1758)}

MATERIAL. CROATIA: $10^{7}$ (SMFM), NE Istria, c. $2 \mathrm{~km} \mathrm{NW}$ of Moscenicka Draga, gorge forest, 2.05.2003, JA \& JM. — GEOR GIA: $2 O^{7} \sigma^{7}, 1$ (ZISP), Batumi, sweeping, 27.06.(no year), coll.? - AZERBAIJAN: 1 ( SZMN), Lenkoran Area, Lenkoran Reserve $\left(38^{\circ} 38^{\prime} \mathrm{N}, 48^{\circ} 47^{\prime} \mathrm{E}\right), 23.05 .2003$, EG; 1 \% (ZMUM), nr. Lenkoran, 6.10.1984, K. Aliev; 1 \& (ZMUM), nr. Bababir Vil., 6.05.1985, PD.

COMMENTS. A Euro-Siberian temperate species [Logunov, Marusik, 2000b].

\section{Evarcha jucunda (Lukas, 1846)}

Figs 75-76, 81-82, 87-88.

MATERIAL. ALGERIA: 1 (MHNG), Djurdjura, Boghni, 11.05.1988, C. Besuchet, I. Löbl \& D. H. Burckhardt; 1 (PCRB), Wilaya Buira, entrance to Aomar et Kadiria, Wadi Isser, c. $200 \mathrm{~m}$ a.s.1., small plantation of Eucalyptus (in litter), 18.08.1988, RB; 1 $\sigma^{7}$ (PCRB), Wilaya Bejaia, E. Adekar, along N34, $800 \mathrm{~m}$ a.s.1., Quercus ilex forest (in litter), 17.04.1982, RB; $1 \mathrm{O}^{7}$ (PCRB), Wilaya Boumerdes, Reghia, $45 \mathrm{~m}$ a.s.l., pitfall traps in degaraded Ouercus suber forest, 13.06.1988, RB; 1 O7 (PCRB), Wilaya Blida, Atlas Blidéen, Meurdja, c. $900 \mathrm{~m}$ a.s.1., nr. fountain in forest, 12.09.1985,
RB; 10 (PCRB), Wilaya Blida, Atlas Blidéen, Chrea, c. $1045 \mathrm{~m}$ a.s.1., pitfall traps in dense Quercus ilex forest, 15.02-21.06.1987, RB; $1 \mathrm{O}^{7}$ (PCRB), Wilaya Tipasa, Bouchaoui, c. $95 \mathrm{~m}$ a.s.1., pitfall traps in Ulmus forest and Eucalypus plantation, 1.01.1987-12.01. 1988, no date, RB; 4 ○ౌ $\sigma^{7}, 3$ 00 (SZMN), Wilaya Tipaza, Sidi Fredj, c. $10 \mathrm{~m}$ a.s.1., pitfall traps in Olea archard, 10.12.198620.12.1987, RB; 1 O , 1 (PCRB), Wilaya Boumerdes, Reghaia, c. $10 \mathrm{~m}$ a.s.1., pitfall traps in Olea europea orchard, 13.06-30.09.1988, RB; 1 O (PCRB), Wilaya Tipasa, Douaouda, Wadi Mazafran, 50 $\mathrm{m}$ a.s.1., glades in forest of Populus alba, 16.12.1986-26.02.1988, RB; $2 \sigma^{7} \sigma^{7}$ (ZMUM), Wilaya Oran, Hadjadz, c. $50 \mathrm{~m}$ a.s.1., in garden, 07-08.1988, RB. - PORTUGAL: $10^{\text {7 }}$ (FMNH), Algodôr (c. $\left.37^{\circ} 45^{\prime} \mathrm{N},-7^{\circ} 48^{\prime} \mathrm{W}\right), 24.05 .2003, \mathrm{PC} ; 1$ ( 1 FMNH), Mértola (c. $\left.37^{\circ} 38^{\prime} \mathrm{N},-7^{\circ} 40^{\prime} \mathrm{W}\right), 16.05 .2003, \mathrm{PC} ; 10^{\prime \prime}$ (ZMUM) \& 2 우 (FMNH), Paúl do Boquilobo Biosphere Reserve (c. $39^{\circ} 23^{\prime} \mathrm{N},-8^{\circ} 32^{\prime} \mathrm{W}$ ), 20.05 11.07.2002, PC; 1 + (ZMUM) \& 1 ( $\left(\right.$ FMNH), Barrenta (c. $39^{\circ} 34^{\prime} \mathrm{N}$, $\left.-8^{\circ} 46^{\prime} \mathrm{W}\right), 7.05-24.09 .2002$, PC. - SPAIN: $2 \bigcirc^{\top} \sigma^{7}, 4$ 우 (MVHN), Valencia, Devesa del Saler (Estibadores \& Polideportivo), 17.0528.06.2004, DD; 1 \%, 1 † (SMFM), La Rabida, 05.1959, J. Büddenbrock; 1 ๆ', 2 90 (NMPC), Catalonia, Deltebre (c. $40^{\circ} 44^{\prime} \mathrm{N}$, $\left.0^{\circ} 50^{\prime} \mathrm{W}\right), 26.04 .2004, \mathrm{LD} ; 2 \sigma^{\prime} \sigma^{7}, 1$ ( $(\mathrm{NMPC})$, Calp, 29.04. 2004 LD; 1 क (NMPC), Salou, 26.04.2004, LD; 1 \%, 1 \% (NMPC), Valensia, Guadalest (c. $38^{\circ} 41^{\prime} \mathrm{N}, 0^{\circ} 11^{\prime} \mathrm{E}$ ), 27.04.2004, LD; $4 \mathrm{O}^{7} \sigma^{\prime \prime}$, 1 \& (NMPC), Guardamar del Segura, 28.04.2004, LD; 3 ○ $\sigma^{7}$ (NMPC), Catalonia, Deltebre (c. 40 $\left.44^{\prime} \mathrm{N}, 0^{\circ} 50^{\prime} \mathrm{W}\right), 26.04 .2004$, LD; 1 or (NMPC), Santa Pola, 28.04.2004, LD; 1 ○ (NMPC), Catalonia, Riumar, 26.04.2004, LD; 1 ऽ (NMPC), Denia, 29.04. 2004, LD. - ITALY: $1 \sigma^{7}, 1$ (OUMNH, bottle 2108), Ospedalethi; $4 O^{7} \sigma^{7}, 1$ (OUMNH), St. Margarita, stones, 20.08.1958, GL; 2 + (SMFM), Sardinia (W part), Camp of Riola Sardo, olive trees with grass, sheep pasture and slightly moist grass, 7.04.1956, HK; $1 \sigma^{7}$ (SMFM, 11/983), Sicily, no date and coll. — GREECE: 1 $\sigma^{7}$ (SMFM), S coast of Peloponnes, Foinikous, lime and sanf cliffs at seashore, 4.06.1998, LM; $10^{7}$ (LMNM), Lesbos, Skala Kallonis, R Potamia $\left(39^{\circ} 12^{\prime} 42^{\prime \prime} \mathrm{N}, 26^{\circ} 10^{\prime} 13^{\prime \prime} \mathrm{E}\right), 9.07 .1997$, M. Hull; 1 우 (PCRG), Thasos, Villa Edem $\left(40^{\circ} 39^{\prime} \mathrm{N}, 24^{\circ} 40^{\prime} \mathrm{E}\right), 10 \mathrm{~m}$ a.s.1., 25.05.2003, AF; 2 ㅇ (SMFM, 4151), Crete, Akrotiri plain, 05.1928, CR; 1 q (SMFM), Crete, c. $3 \mathrm{~km}$ SE of Kaluthiana, pasture nr. stream, Chrysanthemum, Cirsum, Nerium oleander, Myrtus (under and between stones), 19.04.1958, HK; $2 \sigma^{7} \sigma^{7}$ (SMFM), N Crete, Gouves, 7.05.2007, HE \& UK; $1 \sigma^{7}$ (SMFM), Chalkidiki island, Andrea, c. $800 \mathrm{~m}$ a.s.1., forest, 1968, JMS; $1 \sigma^{7}$ (SMFM), Korfu, between Acharavi and Portes $\left(39^{\circ} 48^{\prime} 7.5^{\prime \prime} \mathrm{N}, 19^{\circ} 50^{\prime} 33.1^{\prime \prime} \mathrm{E}\right)$, c. $30 \mathrm{~m}$ a.s.1., Ouercus forest (sieving, under wood and stones), 25.03.2006, AS. - CROATIA: $1 \sigma^{7}$ (MHNG), Istria, nr. Pula, 9-14.07.1982, S. Heimer; 1 フ, 1 q (NMPC), Palagruža, 1-9.09.1997, S. Polak; 1 우 (ZMTU), Dalmatia, Herzegovina, 13.08.1965, PL. - TURKEY: 1 $\sigma^{7}$ (PCRS), Akyaka, Thomsan Villas, on walls of house, 28.05.1996, ARS; 1 क (PCRS), same locality, under stone in pine wood, 1.06.1996, ARS; 1 O (ZMTU), Adapazari Distr., Hendek-Gümüsova, Quercus-Crataegus forest, 10.09.1971, PL; 1 o (SMFM), Büyükada (nr. Istanbul, but the label is illegible), 07-08.1965, coll.?; $1 \sigma^{7}$ (SMFM), Büyükada (the rest of label is illegible), 08.1966, coll.?; $1 \sigma^{7}, 1$ (PCSD), Gulf of Gorkova (c. $4 \mathrm{~km} \mathrm{~N}$ of shore), 27.05.1997, RS; 1 q (PCLJ), Perge, 27.05.2004, N. Jonsson; 1 o (NHMW), Kuşadası (SE border), 11-26.05.1969, F. Spitzenberger; 1 O' (OUMNH), nr. Dalyan, 10-17.08.2008, R. Gabriel \& L. Hedicker.

COMMENTS. A Mediterranean species, known from Algeria and Portugal, eastward to Turkey [Logunov, 2012; Uyar, Uğurtaş, 2012; Helsdingen, 2013, 2014; present data].

This species is very similar to Evarcha eriki from Gran Canaria and to E. patagiata from the east-Mediterranean. The males of all three species are easily distinguishable by the conformation of their tibial apophyses and emboli (Figs 75-80). The females are difficult to separate on the basis of epigyne due to its strong variation (Figs 81-86). The main difference of the female of $E$. patagiata is the presence of the singular 

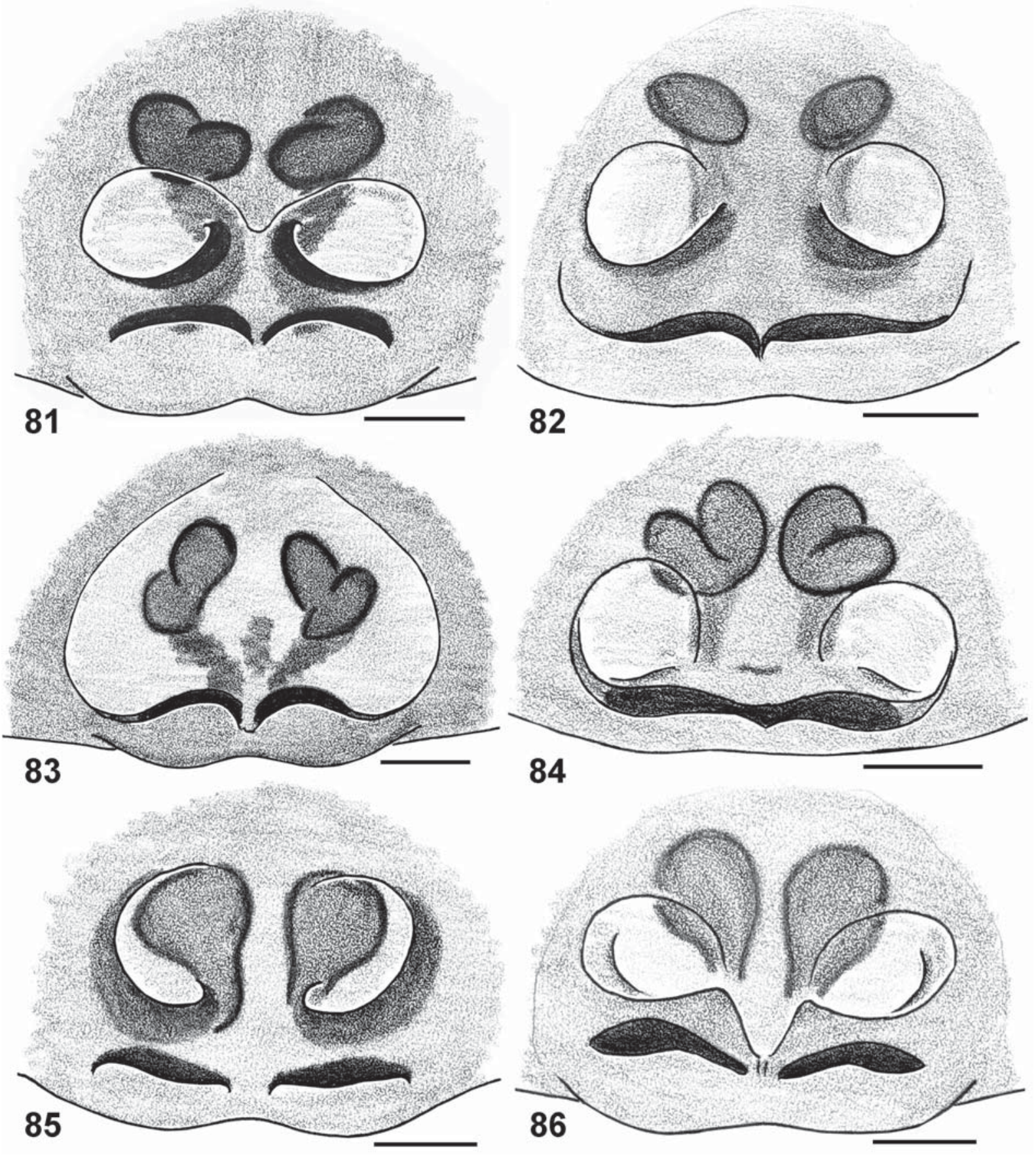

Figs 81-86. Epigynes of Evarcha jucunda (Lukas, 1846) (81 - Portugal; 82 - Italy), E. patagiata (O. Pickard-Cambridge, 1872) (83 - Syria; 84 - Cyprus) and E. eriki Wunderlich, 1987 (85 - Gran Canaria; 86 - paratype), ventral views. Scale bars: $0.1 \mathrm{~mm}$.

Рис. 81-86. Эпигины Evarcha jucunda (Lukas, 1846) (81 - Португалия; 82 - Италия), E. patagiata (O. Pickard-Cambridge, 1872) (83 - Сирия; 84 - Кипр) и E. eriki Wunderlich, 1987 (85 - Гран Канария; 86 - паратип), вид снизу. Масштаб 0,1 мм.

shallow epigynal atrium (=membranous "window" sensu Prószyński [2003]), with the epigynal wings usually merged in a singular transverse plate (Fig. 83). The epigynes both of E. jucunda and of E. ericki possess two separated round shallow atria, with the epigynal wings usually separated from each other as well; by this characater these two species seem unseparable.
Yet, some females of E. patagiata (Fig. 84; from Cyprus) has got the atrium consisting of two separate "windows" and looking almost identical to those of E. jucun$d a$. Some females of E. jucunda may have the epigynal wings merged together (Fig. 82) as those of E. patagiata (Figs 83-84). In such cases, the spermathecal structure is to be examiend for separating the species. 


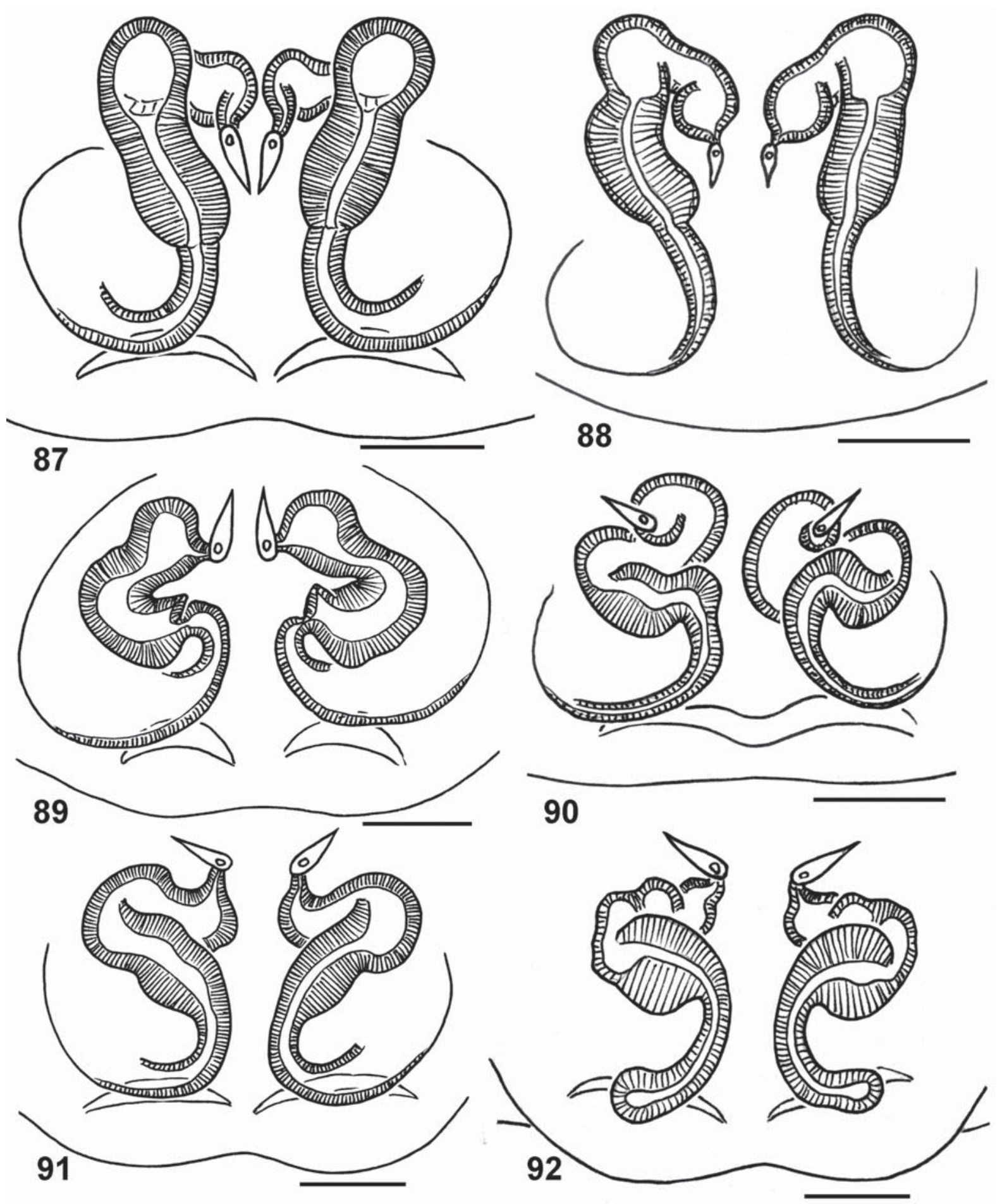

Figs 87-92. Spermathecae of Evarcha jucunda (Lukas, 1846) (87 - Portugal; 88 - Italy), E. patagiata (O. Pickard-Cambridge, 1872) (89 - Syria; 90 - Cyprus) and E.eriki Wunderlich, 1987 (91 - Gran Canaria; 92 - paratype), dorsal views. Scale bars: $0.1 \mathrm{~mm}$. Рис. 87-92. Сперматеки Evarcha јucunda (Lukas, 1846) (87 - Португалия; 88 - Италия), E. patagiata (O. Pickard-Cambridge, 1872) (89 - Сирия; 90 - Кипр) и E.eriki Wunderlich, 1987 (91 - Гран Канария; 92 - паратип), вид сверху. Масштаб 0,1 мм.

The shape, comparative size and the arrangement of the insemination ducts seem to provide reliable diagnostic characters (Figs 87-92): viz., the insemination ducts in E. jucunda are directed anteriad, forming 1-shaped figure; the spermatheca of E. patagiata is Sshaped, with the largest terminal sections(as compared to the related species); the spermathecae of E. eriki is most similar to that of E. patagiata but with the much 
smaller terminal section and with the much stronger sclerotized insemination ducts. Finally, E. eriki seems to be strictly confined to Gran Canaria.

\section{Evarcha laetabunda (C.L. Koch, 1848)}

MATERIAL. CROATIA: $10^{7}$ (SMFM), Lika-Senj, e. Karlobag, Velebit Mts, nr. Baske Ostarije, 900-1200 m a.s.l., hand collecting, 10-11.05.2008, JA \& JM. - RUSSIA: $1 \mathrm{O}^{7}$ (ZISP), Adygeya, nr. Khamyshki, 29.08.1937, AD; $1 \mathrm{O}^{\text {T }}$ (ZISP, A.No. 204), North Caucasus, nr. Saprat (unknown locality), 7.08.1937, AD.

COMMENTS. A trans-Eurasian temperate species [Logunov, Marusik, 2000b].

\section{Evarcha michailovi Logunov, 1992}

MATERIAL. TURKEY: $2 O^{7} O^{7}$ (NMPC), Çağlayan, c. $24 \mathrm{~km}$ $\mathrm{SE}$ of Erzincan $\left(39^{\circ} 36^{\prime} \mathrm{N}, 39^{\circ} 42^{\prime} \mathrm{E}\right)$, valley above waterfall, $23-$ 24.06.2002, MR; 1 + (SMNH), Adana Prov., Belemedik Vil., 18.05. $2003, \mathrm{KK}$

COMMENTS. A Euro-Siberian - Central Asian subboreal species, known from France to SE Transbaikalia and E Mongolia [Logunov, Marusik, 2000b]; the records from Turkey [Yağmur et al., 2009; present data] lie in the southernmost limit of the species' range.

Evarcha nepos (O. Pickard-Cambridge, 1872)

MATERIAL. CYPRUS: $10^{7}$ (LMNM), Paphos, nr. Nikoieia hotel, 5-6.09.1997, CF; 1 ( (LMNM), edge of track by citrus grove $\mathrm{N}$ of Paphos, 6.09.1997, CF.

COMMENTS. Recorded from Cyprus, southern Turkey and Israel only [Prószyński, 2003; Logunov, 2012; present data]. New record for Cyprus.

Evarcha patagiata (O. Pickard-Cambridge, 1872) Figs 77-78, 83-84, 89-90.

MATERIAL. CYPRUS: 1 \& (LMNM), Tróodos, Ágios Nikolás tis Stéyis, c. $8 \mathrm{~km} \mathrm{~S}$ of Agios, streamline across unpaved road, mixed deciduous and coniferous woodland over braken, bramble and Mentha, 9.09.1997, SJ \& CF; 1 O $^{7}$ (LMNM), in deep gorge, rest house, Paphos forest, 8.09.1997, CF. - TURKEY: 1 C (LMNM), Mersin, Narlıkuyu, 15.07.1992, CF; $2 O^{7} O^{7}$ (LMNM), Narlıkuyu, Mersin, garrigue-type vegetation on limestone outcrop, under pine needles and scattered short herbs, 9.06.1993, SJ \& CF 4 우 (LMNM), Içel, Narlıkuyu (36 $\left.26^{\prime} 35^{\prime \prime} \mathrm{N}, 34^{\circ} 04^{\prime} 44^{\prime \prime} \mathrm{E}\right), 29.06$ 1995, SJ \& CF; 1 o (LMNM), Içel, c. $2 \mathrm{~km} \mathrm{~S}$ of Camlidere $\left(36^{\circ}\right.$ $52^{\prime} 12^{\prime \prime} \mathrm{N}, 34^{\circ} 24^{\prime} 37^{\prime \prime} \mathrm{E}$ ), rocky disuded quarry at side of road, dominated by Euphorbia, Mullein Cistus and Rubus, 30.06.1995, SJ \& CF; $50^{\prime 7} \sigma^{7}$ (LMNM), Içel, Camliyayla, Dağ Hotel area $\left(37^{\circ} 10^{\prime} 10^{\prime \prime} \mathrm{N}\right.$, $34^{\circ} 36^{\prime} 02^{\prime \prime E}$ ), c. $1454 \mathrm{~m}$ a.s.1., limestone cliff, 1.07.1995, SJ \& CF; $10^{7}$ (LMNM), Içel, c. $17 \mathrm{~km} \mathrm{~N}$ of Anamur, Alaköprü Bridge (36 $10^{\prime} 38^{\prime \prime} \mathrm{N}, 32^{\circ} 53^{\prime} 51^{\prime \prime} \mathrm{E}$ ), wet flush on road, downward slope to river bank and rocky outcrop, 7.05.1994, SJ \& CF; $10^{7}$ (NHMW), Namrun, 30.05.1964, FR. - SYRIA: $1 \sigma^{7}$ (NMPC), NW Syria, Mashtal Helu nr. Homs, 30.03-3.04.2001, MR; $10^{7}$ (NMPC), NW Syria, castle Qalaat al Hosn nr. Homs, 3-4.04.2001, MR; 1 9 (NMPC), NW Syria, Al Haffeh nr. Latakia, 13.04.2001, MR.

COMMENTS. An eastern Mediterranean species, recorded to date from Greece, Turkey, Cyprus, Israel and Syria [Prószyński, 2003; Russell-Smith et al., 2011; Logunov, 2013; present data]. New record for Turkey.

Having examined numberous males and females of this species from Cyprus, Turkey and Syria, I agree with the opinion of Prószyński [2003: 59] that the name of E. syriaca Kulczyński, 1911 should be consid-

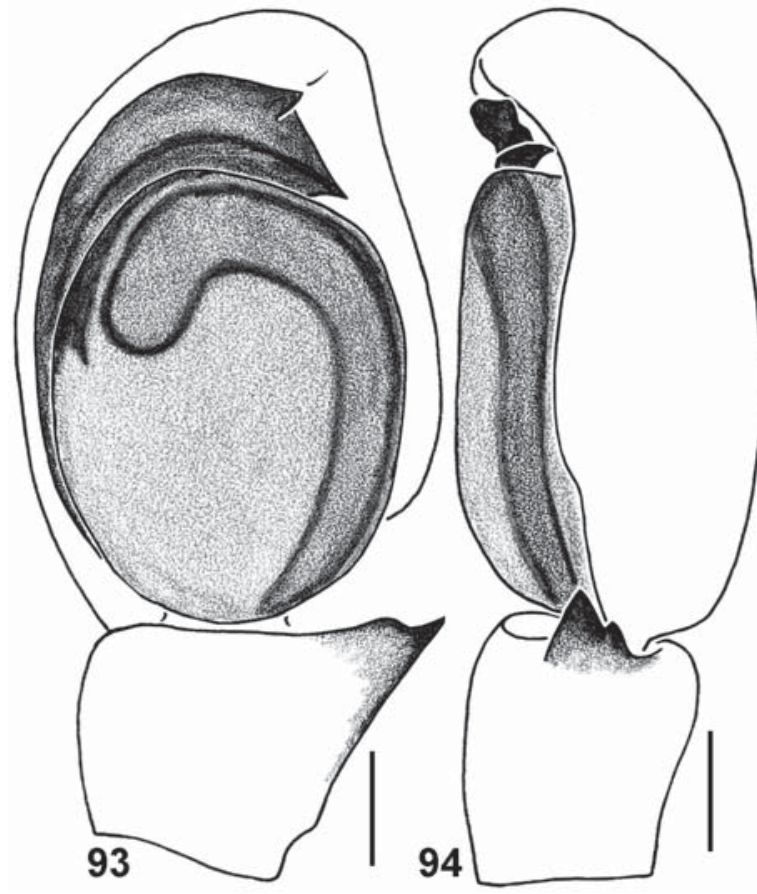

Figs 93-94. Male palp of Evarcha pileckii Prószyński, 2000 (Syria): 93 - ventral view; 94 - retrolateral view. Scale bars: 0.1 $\mathrm{mm}$

Рис. 93-94. Пальпа самца Evarcha pileckii Prószyński, 2000 (Сирия): 93 - вид снизу; 94 - вид сзади-латерально. Масштаб $0,1 \mathrm{Mм}$

ered a junior synonym of E. patagiata; the formal synonymization was done by Prószyński [2014]. The record of uncertain Evarcha species from Syria based on the old male specimen having white setae on its cymbium (see Prószyński [2003: 63, figs 231-232]) should also be referred to E. patagiata.

See also above under the 'Comments' of E. jucunda.

\section{Evarcha pileckii Prószyński, 2000 Figs 93-94.}

MATERIAL. SYRIA: $1 \sigma^{7}$ (ZMUM), NW Syria, castle Qalaat al Kahf nr. Qadmus, 5-7.04.2001, MR.

COMMENTS. Recorded from Israel and Syria only [Prószyński, 2003; present data]. New record for Syria.

This species remains poorly studied and is known from the male only.

\section{Habrocestum egaeum Metzner, 1999}

MATERIAL. GREECE: 1 (ZMTU), Rhodos City, stone wall - sand dune, 27-28.05.1973, PL; 2 오 (SMFM), Crete, Meskla, 06.1926, CR; 4 OT $^{7}, 3$ 오 (SMFM), Crete, Topolia, 06.1926, CR; 2 우 (SMFM), Aptera, no date; $10^{7}$ (SMFM, 4151), Crete, Akrotiri plain, 05.1928, CR; 1 O $^{2}$ (SMFM, 4152), Crete, Aptera, 30.05. 1928, CR; 1 (SMFM), Crete, W of Camaris, 600-800 m a.s.l., slope, rocky, Phrygana, some Quercus coccifera, Juniperus, 6.04 . 1958, HK; 1 (SMFM), Crete, S-side of Lake Curna, slope scree, Cirsium, reed, yellow Laminacea, 15.04.1958, HK; 1 ㅇ (SMFM), Crete, c. $25 \mathrm{~km}$ SE of Rethymnon. Archea Eleftherna, 17.05.2008, HE \& UK; $10^{7}, 1$ ㅇ (SMFM), C-Crete, Magarikari, 7.05.2009, KE \& HE; $1 \mathrm{O}^{\top}$ (SMFM), Dadekanes, Karpathos, NE Piles, c. $330 \mathrm{~m}$ 
a.s.1., dry valley with bushes of Pistacea sp., 21.09.1963, JMS; o (SMFM), Kelempek Mts, summit region, $900 \mathrm{~m}$ a.s.1., 8.04.1968, JMS; 1 O' (SMFM), Island Kos, Kastell, 28.04.1968, JMS. TURKEY: $1 \sigma^{7}$ (PCRS), c. $6 \mathrm{~km}$ from Torba, road to Yalikavak, in eroded area, 2.06.1997, ARS.

COMMENTS. Recorded from Greece (mainland and Crete) and Turkey only [Metzner, 1999; Logunov, Chatzaki, 2003; present data]. New record for Turkey.

Habrocestum graecum Dalmas, 1920

MATERIAL. GREECE: $2 \sigma^{7} \sigma^{7}, 2$ OP (SMFM), S coast of Peloponnes, Foinikous, lime and sand cliffs at seashore, 4.06.1998, LM; $10^{7}, 1$ + (SMFM), S coast of Peloponnes, Ligia, beach, dunes and adjacent cultural landscape, 1.06.1998, LM

COMMENTS. The mainland Greece only [Metzner, 1999; Bosmans, Chatzaki, 2005; present data].

Habrocestum papilionaceum (L. Koch, 1867)

MATERIAL. GREECE: $10^{7}$ (SMFM, 11516), 'Tripolitza', no date and collector. - TURKEY: $5 \mathrm{O}^{7} \mathrm{O}^{7}, 1$ (PCRS), c. $1 \mathrm{~km} \mathrm{~W}$ of Yalikavak, nr. Bodrum, under stone in meadow and maquis, 28.053.06.1997, ARS; 2 O $^{7}$ O $^{7}$ (NHMW), Kupadasý (SE-edge), Formolfalla, 11-26.05.1969, F. Spitzenberger.

COMMENTS. Recorded from Greece and Turkey only [Karol, 1967; Metzner, 1999; Russell-Smith et al., 2011; present data].

Hasarius adansoni (Audouin, 1826)

MATERIAL. ALGERIA: $1 \sigma^{7}$ (PCRB), Wilaya Alger, Beaulieu, c. $50 \mathrm{~m}$ a.s.l., in garden, no date, RB. - SPAIN: 1 + (NMPC), Barcelona, 25.04.2004, LD. - GREECE: $1 O^{7}$ (SMFM), S Crete, Plakias, 21.07.2009, HE \& UK. - EGYPT: 1 (NHMW), Cairo, 25.04.1983, HEH. - OMAN: $1 \mathrm{O}^{7}$ (NHMW), nr. Tawi Ateyr cave, 19.01.2001, Sattmann et al. - SYRIA: $1 O^{\prime}$ (NMPC), NW Syria, Latakia, 16.04.2001, MR.

COMMENTS. A cosmopolitan species (in warm climate and green houses), also recorded from southern Europe and the Mediterranean [Helsdingen, 2014]. New records for Oman, Egypt and Syria.

Heliophanillus fulgens (O. Pickard-Cambridge, 1872) MATERIAL. EGYPT: $1 \sigma^{7}$ (SMFM), Luxor, island on Nile, bamboo, 17.11.1996, PJ. - SYRIA: 1 O' (NMPC), NW Syria, Aln al Zara nr. Idlib, canyon of Oronthes, 28.03.2001, MR; 1 o (SMFM), Hama Prov., al-Ghab, street from Saqalbiya to SW valley, road ditches $1 \mathrm{~km} \mathrm{~W}$ of the bridge of al-Hansa, 30.03.1979, RK. - JORDAN: 1 O $^{\text {T }}$ (MHNG), east part, Wadi Schaib, 6.04.1967, J. \& S. Klapperich.

COMMENTS. An eastern Mediterranean species, known from Greece (Crete only) and Egypt [Metzner, 1999; Logunov, Chatzaki, 2003], throughout the Near East [Prószyński, 2003], to central and southern Iran [Logunov et al., 2002, 2007], southward to Yemen and the UAE [Wesołowska, van Harten, 1994, 2007, 2011]. New record for Jordan.

\section{Heliophanus agricola Wesołowska, 1986} Figs 100-101.

MATERIAL. ALGERIA: 1 9 (PCRB), Wilaya Oran, Daiet El Bragat along W18, c. $100 \mathrm{~m}$ a.s.1., among dry Salicornia and other herbs, 25.04.1984, RB. - SPAIN: 1 + (MMUM, G7512.1913), Almeria, under stones in abandoned field near Salinas Viejas, 22.03.1979, ED

COMMENTS. A west-Mediterranean species, confined to the Iberian Peninsula and northern Algeria [Wesołowska, 1986; present data].
Heliophanus agricoloides Wunderlich, 1987 Figs 95-99, 102-103.

Heliophanus agricoloides Wunderlich, 1987: 272, figs 686ad (D $\sigma^{7} O$; holotype $\sigma^{7}$ from Naturmuseum und Forschungsinstitutt Senckenberg, Frankfurt am Main, Germany; examined).

TYPE: Holotype $\sigma^{7}$ (SMFM, 37287) from Spain, the Canaries, Gran Canaria, nr. San Agostin, VII, J. Wunderlich. - Paratypes: 1 $\sigma^{7}, 1$ क (SMFM, 37301), together with the holotype.

MATERIAL. SPAIN: $5 \sigma^{7} \sigma^{7}, 3$ of (OUMNH), The Canaries, Gran Canaria, Chavco of Maspalomas, beaten palm for sedum, 31.07.1957, GL.

COMMENTS. Restricted to Gran Canaria [Wesołowska, 1986; Wunderlich, 1987; present data].

In the original description, Wunderlich [1987: fig. 686b] did not show the bent tip of the embolus (cf. Fig. 96). H. agricoloides is very similar to $H$. agricola (Figs 100-101) and $H$. fuerteventurae (see above; Figs 13$19)$, especially the females. The conformation of the spermathecae (viz., arrangement and proportions of the inseminations ducs) is a reliable diagnostic character for distinguishing the females of these three species.

\section{Heliophanus apiatus Simon, 1868}

MATERIAL. SPAIN: $1 \sigma^{7}$ (MVHN), Valencia, Benimodo, Nacimiento Rio Verde, 12.10.2004, SM; $1 \sigma^{T}$ (NMPC), Guardamar del Segura, 28.04.2004, LD; 1 O$^{7}$ (NMPC), Blanes, 1.05.2004, LD; $1 \sigma^{\top}$ (NMPC), Valencia, Guadalest (c. $\left.38^{\circ} 41^{\prime} \mathrm{N}, 0^{\circ} 11^{\prime} \mathrm{E}\right), 27.04$. 2004, LD; 10 O $^{7}, 7$ 웅 (NMPC), Denia, 29.04.2004, LD. ITALY: $1 \sigma^{7}$ (SMFM), Sicily, c. $1 \mathrm{~km} \mathrm{~S}$ of Ficuzza, forest of Pinus piaster, on clearing with moist grassland and Asphodelus (under stones), $700 \mathrm{~m}$ a.s.1., 12.04.1957, HK; $1 \sigma^{7}$ (SMFM), Sicily, nr. Ficuzza, N-slope at northern Rocca Busambra, moist grassland with Crataegus, Rubus, Prunus, Berris perennis and Cirsium (under stones), $800 \mathrm{~m}$ a.s.1., 11.04.1957, HK; $1 \mathrm{O}^{7}$ (SMFM), Sicily, c. $2 \mathrm{~km} \mathrm{~W}$ of Castelbuono, grainfield at W-slope (under stones), 29.03.1957, HK; 2 O $^{7} \mathrm{O}^{7}$ (SMFM), Sicily, c. $2 \mathrm{~km}$ W of Castelbuono, stony SE-slope, moist grainfields with Fraxinus ornus (under stones), 31.03.1957, HK; 1 O $^{7}$ (SZMN), Sicily, R.N.O. Zingaro, nr. Trapani, pitfall traps, 17-31.09.2001, F. di Franco; 1 क (SZMN), same locality, 29.05.2003, F. di Franco; $10^{7}$ (SMFM), Sardinia (SW part), c. $2 \mathrm{~km} \mathrm{~S}$ of Gonnosfanadiga, steep E-slope, cultivated land, olive trees, with dense grass and clover (under stones), 6.04.1956, HK; 1 + (SMFM), Sardinia (W part), c. 2 km S of Guglieri, hills, 500-580 m a.s.1., N-slope, pastures with stonewalls, Castanea, Euphorbia, Rubus fruticosa (under stones), 14.04. 1956, HK.

COMMENTS. Recorded from the Iberian Peninsula to Italy (Sicily) [Wesołowska, 1986: map 897; Ijland, Helsdingen, 2014; present data].

\section{Heliophanus auratus (C.L. Koch, 1835)}

MATERIAL. ALBANIA: $20^{7} \sigma^{7}, 1$ ( (SMFM), 'IPEK, Bjelopolie', 06.1921, coll.? - MACEDONIA: 2 99 (OUMNH), Geugelya, 10.07.1956, GL. - GREECE: 1 + (SMFM), Crete, Knosos, 06.1926, CR; 1 P (SMFM), Kavala, Rentina Valley, 19.08.1971, RK. CROATIA: $1 \mathrm{O}^{7}$ (SMFM), Primorije-Gorski kotar, nr. Delnice, c. $800 \mathrm{~m}$ a.s.l., beech forest (hand collecting from limestone), 28.04 . 2008, JA \& JM. — TURKEY: 1 O$^{\top}, 2$ क 9 (LMNM), Antalya, c. 31 $\mathrm{km} \mathrm{N}$ of Alanya, Akcali Daglari, 19.07.1992, CF; 3 O $^{7} \sigma^{7}, 1$ 우 (LMNM), Niðde $\left(38^{\circ} 19^{\prime} 37^{\prime \prime} \mathrm{N}, 34^{\circ} 14^{\prime} 34^{\prime \prime} \mathrm{E}\right)$, c. $33 \mathrm{~km}$ E of Aksaray, Selime Bridge, disturbed river bank and herb-rich limestone cliff base, 10.05.1994, SJ \& CF; $20^{7} \sigma^{\top}$ (LMNM), Bolu, c. $21 \mathrm{~km} \mathrm{E}$ of Akçakoca $\left(41^{\circ} 06^{\prime} 27^{\prime \prime} \mathrm{N}, 31^{\circ} 15^{\prime} 54^{\prime \prime} \mathrm{E}\right)$, sparse mixed weeds on road alope above snore, wet field with Juncus adjacent to stream,

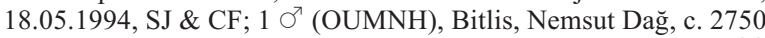
$\mathrm{m}$ a.s.l., lake in garden (under stones), 12.08.1956, GL; 3 우 (NMPC), Yakaavsar nr. Aksu, Dedegol Mts, Pinus forest below Raham Mt., c. 2206 m a.s.1., 10.07.2003, MR; $2 \sigma^{7} \sigma^{7}, 1$ ( (NMPC), 

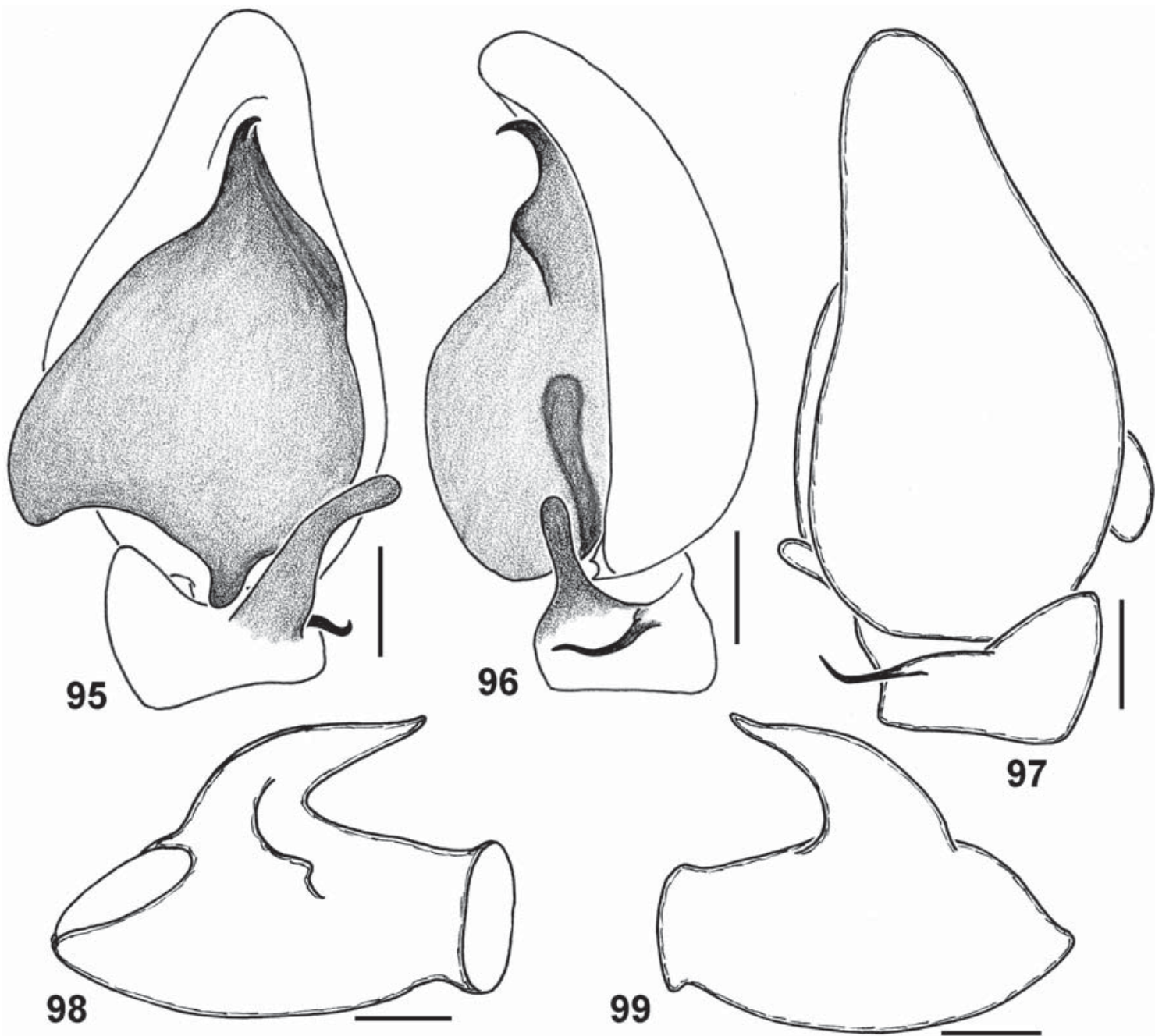

Figs 95-99. Male palp of Heliophanus agricoloides Wunderlich, 1987 (Gran Canaria, Chavco of Maspalomas): 95 — bulbus, ventral view; 96 - ditto, retrolateral view; 97 - ditto, dorsal view; 98 - male palpal femur, prolatearal view; 99 - ditto, retrolateral view. Scale bars: $0.1 \mathrm{~mm}$.

Рис. 95-99. Пальпа самца Heliophanus agricoloides Wunderlich, 1987 (Гран Канария, Маспаломас): 95 - бульбус, вид снизу; 96 - тоже, сзади-латерально; 97 - тоже, вид сверху; 98 - бедро пальпы самца, вид спереди-латерально; 99 - тоже, вид сзадилатерально. Масштаб 0,1 мм.

Çağlayan (c. $24 \mathrm{~km} \mathrm{SE}$ of Erzincan; $39^{\circ} 36^{\prime} \mathrm{N}, 39^{\circ} 42^{\prime} \mathrm{E}$ ), valley above waterfall, 23-24.06.2002, MR; $1 \mathrm{O}^{\mathrm{T}}$ (ZMTU), Izmir Distr., Yamanlar Daği, in pine litter, 24.05.1973, PL; 1 \% (NHMW), Tekketal, SW Akșehir, 1100-1200 m a.s.1., pine forest with Quercus coccifera, Corylus, etc., 11.06.1967, JG - RUSSIA: 2 O $^{2} \mathrm{O}^{7}, 5$ 우 (ZMUM), Astrakhan' Region, Lishansky Distr., E of Basinskaya station $\left(46^{\circ} 07^{\prime} 55^{\prime \prime} \mathrm{N}, 47^{\circ} 13^{\prime} 05^{\prime \prime} \mathrm{E}\right), 18.05 .2012$, AG; $10^{\prime}$ (MMUM), Stavropol Territory, Beshpagir Vil., in kitchen garden, 29.08.2013, DL. - ARMENIA: $10^{7}$ (ZISP, A.No. 617), Elenovka on Gokcha Lake, 22.07.1922, AD. - AZERBAIJAN: 2 우 (ZMUM), Evlakh Distr., nr. Karadein Vil., 23.08.1977, PD; 1 क (SZMN), Naxçivan, Shakhbuz Distr., nr. Kemyur, 1500 m a.s.l., 9.07.1988, PD

COMMENTS. A Euro-Siberian - Central Asian temperate species [Logunov, Marusik, 2000b].

Heliophanus canariensis Wesołowska, 1986

MATERIAL. SPAIN: $1 \sigma^{7}$ (MHNG), the Canaries, El Hierro Island, Maseta de Nisdafe, c. $2 \mathrm{~km} \mathrm{~N}$ of Mirador de Jinama, under rocks making the wall, $1100 \mathrm{~m}$ a.s.1., 2.06.2000, CL.
COMMENTS. Restricted to the Canary Islands [Wesołowska, 1986; Wunderlich, 1992; present data].

Heliophanus creticus Giltay, 1932

MATERIAL. GREECE: 3 O Oొ $^{\top}$ (SMFM, $4151 \&$ 1986), Crete, Akrotiri plain, 05.1928, CR; 1 + (SMFM), Crete, c. $3 \mathrm{~km} \mathrm{SE}$ of Kaluthiana, W-slope, cultivated land with ruin and stonewalls, Olea europea, Quercus ruber, low grass (under stones), c. $50 \mathrm{~m}$ a.s.l., 19.04.1958, HK; $1 \sigma^{7}$ (SMFM), NE Crete, c. $12 \mathrm{~km} \mathrm{~S}$ of Sitia, Macchia, Cystus stony and dry karts, 22.03.1958, HK; $1 \mathrm{O}^{7}$ (SMFM), Crete, S of Zagos, steep SE-slope, stony, dense Phrygana (under stones), 5.04.1958, HK; 1 ( (SMFM), Crete, c. $3 \mathrm{~km}$ SE of Kaluthiana, pasture nr. stream, Chrysanthemum, Cirsum, Nerium oleander, Myrtus (under and between stones), 19.04.1958, HK; 1 $\sigma^{7}$ (SMFM), Crete, S-side of Lake Curna, slope scree, Cirsium, reed, yellow Laminacea, 15.04.1958, HK; $1 \sigma^{\top}$ (SMFM), between Monastir Toplou and Vai $\left(35^{\circ} 14^{\prime} 8.5^{\prime \prime} \mathrm{N}, 26^{\circ} 13^{\prime} 27.1^{\prime \prime} \mathrm{E}\right), 168 \mathrm{~m}$ a.s.1., open and dry phrygana, under stones, 21.03.2007, AS.

COMMENTS. Recorded from Crete only [Wesołowska, 1986; Metzner, 1999; Logunov, Chatzaki, 2003; present data]. 


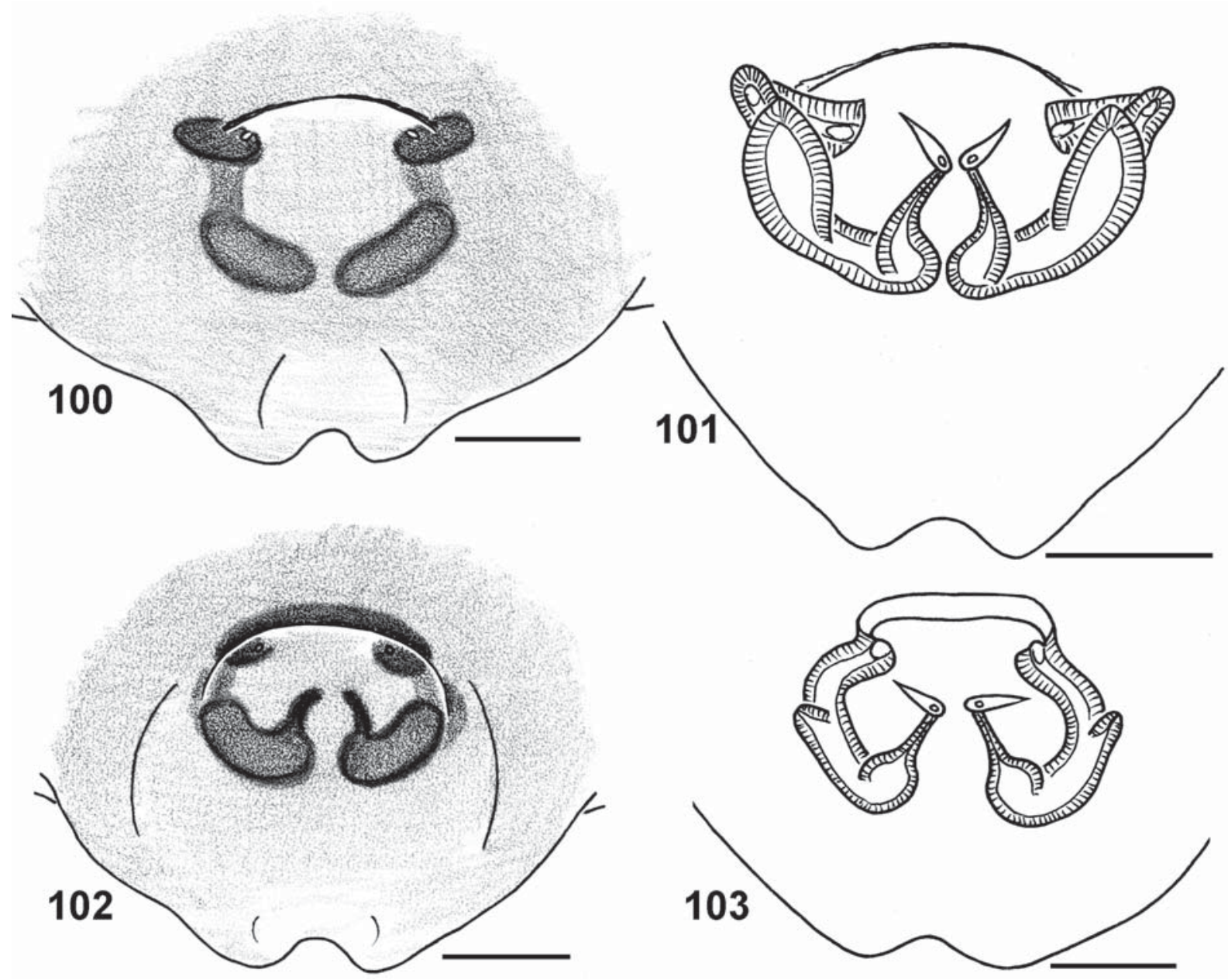

Figs 100-103. Female copulatory organs of Heliophanus agricola Wesołowska, 1986 (100-101; Spain, Almeria) and H. agricoloides Wunderlich, 1987 (102-103; Gran Canaria, Chavco of Maspalomas): 100, 101 - epigyne, ventral view; 101, 103 — spermathecae, dorsal view. Scale bars: $0.1 \mathrm{~mm}$.

Рис. 100-103. Копулятивные органы самок Heliophanus agricola Wesołowska, 1986 (100-101; Испания, Алмерия) и $H$. agricoloides Wunderlich, 1987 (102-103; Гран Канария, Маспаломас): 100, 102 - эпигина, вид снизу; 101, 102 - сперматека, вид сверху. Масштаб 0,1 мм.

Heliophanus cupreus (Walckenaer, 1802)

MATERIAL. ALGERIA: $1 \sigma^{7}$ (PCRB), Wilaya El Tarf, El Kala, W bank of Lac Tonga, c. $10 \mathrm{~m}$ a.s.l., wet meadows along lake, 27.03.1988, RB. - PORTUGAL: $40^{7} \sigma^{\prime \prime}, 5$ 우 (FMNH), Paúl do Boquilobo Biosphere Reserve (c. $39^{\circ} 232 \mathrm{~N},-8^{\circ} 322 \mathrm{~W}$ ), 11.06 18.09.2002, PC. - SPAIN: $1 \mathrm{O}^{7}$ (MHNG), Zamora, nr. Sandin de Carballeda, 17.07.1975, PH; $10^{7}$ (NMPC), Besalú, 2.05.2004, LD - FRANCE: $1 \mathrm{O}^{7}$ (SMFM), Corsica, Col de Verde, c. $1060 \mathrm{~m}$ a.s.1., mixed forest, 9.05.1982, JMS. - ITALY: 1 ㅇ (SMFM), Sardinia (NW part), camp of Scala di Giocca, marshy stream, with Rubus fruticosus at banks (in grass), 18.04.1956, HK. - SLOVENIA: 1 क (SMFM), Slioica, Mt nr. Zirknitz, 800-1000 m a.s.l., 17.07.1974, JMS. - GREECE: 1 (SMFM), Chalkidiki, Cholomon, c. 850 m a.s.1., 19.08.1971, RK. - TURKEY: 1 + (PCRS), c. $8 \mathrm{~km} \mathrm{~W}$ of Koycegiz, sycamore woodland, 5.06.1996, ARS; 2 $\mathrm{O}^{7} \mathrm{O}^{7}, 1$ ( 1 (NMPC), nr. Pazar, c. $24 \mathrm{~km} \mathrm{~W}$ of Tokat $\left(40^{\circ} 16^{\prime} \mathrm{N}, 36^{\circ}\right.$ $\left.17^{\prime} \mathrm{E}\right)$, Ballica, nr. Mağarasi cave, 29.06-1.07.2002, MR; 2 우 (NMPC), Çağlayan, c. $24 \mathrm{~km} \mathrm{SE}$ of Erzincan $\left(39^{\circ} 36^{\prime} \mathrm{N}, 39^{\circ} 42^{\prime} \mathrm{E}\right)$, valley above waterfall, 23-24.06.2002, MR; $10^{3}$ (NHMW), c. 8 $\mathrm{km} \mathrm{S}$ of Tokat, c. $900 \mathrm{~m}$ a.s.l., oak forest with Carpinus, Pinus, etc., 5.06.1967, JG, FR \& AR; 2 우 (NHMW), Kizilcahaman, 1000-1200 m a.s.1., thicket of fruit trees, 27-28.05.1967, JG, FR \& AR; 1 + (NHMW), Namrun, pine forest, 12.05-5.06.1964, FR;
10 오 (BMNH), Trabzon, Hamsiköy, c. 1250 m a.s.l., on rocks, herb litter and under stones on open hillside and in forest, 2125.08.1958, coll.? - RUSSIA: 1 o (ZISP, A.No. 204), North Caucasus, nr. Saprat (unknown locality), 7.08.1937, AD. - ABKHAZIA: 1 (ZMUM), nr. Sukhum, left bank of Kelasuri River (c. $3 \mathrm{~km}$ upstream of its mouth), under stones, 11.06.2004, EM; 1 $\mathrm{O}^{7}$ (ZMUM), nr. Sukhum, Kelasuri, orchards (hand collecting), 711.06.2004, EM. - GEORGIA: 1 \& (ZISP, A.No. 301-970), Tsablana, 1100 m a.s.1, sweeping over Rododendron, 22.06.1969, EP; 1 ㅇ (ZISP, A.No. 491), [Adzharia], Batumi, in grass, 20.08.1914, A. Bykova; 1 ( (ZISP), same locality, Batumi, sweeping, 27.06.(no year), coll.?; 1 + (ZISP), same republic, Tsoniarisi, $400 \mathrm{~m}$ a.s.1., sweeping, 18.06.1969, EP. - ARMENIA: 1 \% (ZISP, A.No. 5101931), Dilizhan, 4.08.1931, EP. - AZERBAIJAN: 1 ㅇ (SZMN), Lenkoran Area, Lenkoran Reserve $\left(38^{\circ} 38^{\prime} \mathrm{N}, 48^{\circ} 47^{\prime} \mathrm{E}\right), 23.05 .2003$, EG; 2 우 (ZMUM), same area, nr. Kirovsk (now Istisu), 24.06. 1985, PD.

COMMENTS. A European - Central Asian temperate species [Helsdingen, 2014], eastward to the Urals and southward to Iran and Afghanistan [Logunov, Marusik, 2000b]. 
Heliophanus decoratus L. Koch, 1875

MATERIAL. CYPRUS: 1 (PCRS), Akrotiri Peninsula, salt marsh, under stones, 30.04.2013, coll.? - EGYPT: $1 \sigma^{\text {th }} 1$ क

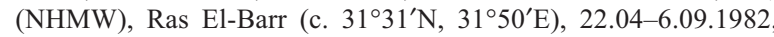
HEH. - SYRIA: 2 +o (SMFM), Dayr az-Zor Prov., $23 \mathrm{~km} \mathrm{NW}$ of Dayr az-Zor, Nahr al Furat (=Euphrates), road bridge between km 290th and 295th to Aleppo, salt marsh, 15.03.1979, RK

COMMENTS. A Mediterranean species, known from Algeria [Wesołowska, 1986: map 905], throughout N Africa and the Near East [Prószyński, 2003], to SW Iran [Logunov et al., 2002]. New record for Cyprus.

Heliophanus dubius C.L. Koch, 1835

MATERIAL. GEORGIA: 1 + (ZMUM), Surami (=Rikoti) Pass, c. $1000 \mathrm{~m}$ a.s.l., Fagus-Alnus-Castanea-Rhododendron forest, litter \& under stones, 14.04-17.05.1983, SG.

COMMENTS. A trans-Eurasian temperate species [Logunov, Marusik, 2000b].

Heliophanus dunini Rakov et Logunov, 1997

MATERIAL. TURKEY: $20^{7} \sigma^{7}$ (NHMW), Akșehir, Sültandağ, 23-31.05.1960, FR. - UKRAINE: $1 O^{7}$ (ZMUM), Kherson Region, nr. Stanislav, bay shore, 17.05.2001, V. Martynov. - AZERBAIJAN: $10^{7}, 2$ 아 (ZISP, A.No. $\left.186 \& 454-1935\right)$, c. $50 \mathrm{~km} \mathrm{~S}$ of Yevlakh, nr. Martushevany, 06-11.1935, P.A. Veltishev.

COMMENTS. Recorded from Turkey, Ukraine, Azerbaijan, N Iran and NW Kazakhstan [Rakov, Logunov, 1997; Logunov, Guseinov, 2002; Logunov et al., 2002; present data]. New records for Turkey and Ukraine.

\section{Heliophanus edentulus Simon, 1871}

MATERIAL. GREECE: 1 ( SMFM), Kelempek Mts, between Avas and Esimi, 05.1968, JMS. - CYPRUS: 1 (MHNG), Larnaca, 27.07.1977, C. Besuchet. - TURKEY: $1 \mathrm{O}^{\top}$ (SMFM), c. $6 \mathrm{~km}$ SW of Iznik, Iznik Gölü, S shore, no date, coll.?; 1 + (SMFM), Iznik Gölü, N shore, c. $26 \mathrm{~km}$ E of Orhangazi, no date, coll.? ISRAEL: $2 \sigma^{7} \sigma^{7}, 1$ (NMPC), Sede Boqer, Negev desert, between Beer Sheva and Mitzpe Ramon, 6-29.05.2003, MR. - SYRIA: 1 $\mathrm{O}^{7}$ (NMPC), NW Syria, castle Qalaat al Hosn nr. Homs, 3-4.04.2001, MR; $10^{7}$ (SMFM), Homs Prov., nr. Qattinah, fields between village and dam., 11.03.1977, RK; 4 우 (SMFM), Homs Prov., Homs Lake nr. Qattina, lakeside at the western end of the dam, Orintes outflow, seasonally wet floodlands, 26.03.1977, RK; 1 o (SMFM), Halab, NW of Aleppo, Nahr' Afrin, upstream of the bridge nr. Afrin, 19.03.1977, RK; 1 क (SMFM), Hama Prov., alGhab, W-slope, $12 \mathrm{~km} \mathrm{~S}$ of Jisr Ash-Shughur, ditches nr. road, 22.03.1979, RK. — IRAN: 1 O $^{7}$ (MHNG), Tehran, Moa'llem Kelayeh $\left(36^{\circ} 26^{\prime} \mathrm{N}, 50^{\circ} 27^{\prime} \mathrm{E}\right), 3.07 .1975$, A. Senglet; $10^{7}$ (MMUM), Sepid dasht, 2.08.2012, SZ.

COMMENTS. A Mediterranean species, known from Algeria [Wesołowska, 1986: map 896], throughout N Africa, Greece [Metzner, 1999] and the Near East [Karol, 1967; Prószyński, 2003; Coşar et al., 2014], to central Iran [Logunov et al., 2002]. New records for Cyprus and Syria.

\section{Heliophanus encifer Simon, 1871}

MATERIAL. CYPRUS: $2 \sigma^{7} \sigma^{7}, 1$ (PCMA), Gialia, 25.03.1998, MA; $1 \sigma^{7}$ (PCDM), Dali, Lunbourena, in field, spring 2004, DMC; 1 + (PCDM), house in Dali, in garden, spring 2004 DMC.

COMMENTS. It is the east-Mediterranean species known from Corsica to Israel [Wesołowska, 1986: map 900; Prószyński, 2003; Helsdingen, 2014; present data].
Heliophanus equester L. Koch, 1867

MATERIAL. GREECE: 2 $0^{7} O^{7}$ (LMNM), Lesbos, Vatera $\left(39^{\circ} 01^{\prime} 13^{\prime \prime} \mathrm{N}, 26^{\circ} 10^{\prime} 53^{\prime \prime} \mathrm{E}\right), 20.04 .1997, \mathrm{CF} \& \mathrm{SJ} ; 1$ 오 (PCRG), Thasos, Thasos shell valley fen $\left(40^{\circ} 39^{\prime} \mathrm{N}, 24^{\circ} 40^{\prime} \mathrm{E}\right)$, c. $25 \mathrm{~m}$ a.s.l., 26.05.2003, AF; 1 क (SMFM), Saloniki, no date and collector name; $1 O^{7}$ (SMFM), Crete, Knosos, 06.1926, CR; 1 O $^{7}$ (SMFM, 5152), Saloniki, no date and collector name; 1 ( (SMFM), Chalkidiki, Kassandra, N Chanioti, 12-15.08.1971, RK; 1 o (SMFM), Crete, c. $3 \mathrm{~km}$ SE of Kaluthiana, pasture nr. stream, Chrysanthemum, Cirsum, Nerium oleander, Myrtus (under and between stones), 19.04.1958, HK; 3 O $^{7} \sigma^{7}$ (SMFM), N Crete, Gouves, 2-4.05.2008, HE \& UK; 1 O (SMFM), S coast of Peloponnes, Ligia, beach, dunes and adjacent cultural landscape, 1.06.1998, LM; 1 \% (SMFM), S coast of Peloponnes, Foinikous, cultural lands, 4.06. 1998, LM. - TURKEY: 1 + (PCRS), Akyaka, nr. Cinan Beach, under stones in pine wood, 30.05.1996, ARS; $10^{7}$ (PCRS), meadow c. $1 \mathrm{~km} \mathrm{~W}$ of Yalikavak, nr. Bodrum, under stone, 28.05.1997, ARS; $1 O^{7}$ (LMNM), Antalya, near Imamli, c. $3 \mathrm{~km} \mathrm{~W}$ of Yesiloz, vegetated upper beach next to Cag Motel, 8.06.1993, CF; 1 ㅇ (LMNM), Içel, c. $2 \mathrm{~km} \mathrm{~S}$ of Çamlidere (36 $\left.52^{\prime} 12^{\prime \prime} \mathrm{N}, 34^{\circ} 24^{\prime} 37^{\prime \prime} \mathrm{E}\right)$, rocky disuded quarry at side of road, dominated by Euphorbia, Mullein Cistus and Rubus, 30.06.1995, SJ \& CF; 1 O' (ZMUM), Antalya, Kemer, pine forest, 50-100 m a.s.1., 28.04.2001, KM; 1 + (NMPC), Tunceli $\left(39^{\circ} 06^{\prime} \mathrm{N}, 39^{\circ} 33^{\prime} \mathrm{E}\right)$, Munsur river, 22.06.2002, MR; 1 O', 3 우 (ZMTU), Izmir Distr., Yamanlar Vil., in dry meadow on a slope, 25.05.1973, PL, F. Önder \& E. Pehlivan; 1 ㅇ (ZMTU), Izmir Distr., Kersiyaka, stone bed along a small river, 24.05-30.07.1973, PL \& F. Önder; $1 \overbrace{}^{7}, 1$ (PCSD), Gulf of Gorkova, behind beach Akyaka, 27.05.1997, RS; 1 (PCSD), Gulf of Gorkova (c. $4 \mathrm{~km} \mathrm{~N}$ of shore), 27.05.1997, RS. - ARMENIA: 2 $\mathrm{O}^{7} \mathrm{O}^{7}, 7$ 우 (ZISP, A.No. 317-09), Yerevan Area, 'station Negram' (unknown locality), 19.05.1909, N. Bryansky. - AZERBAIJAN: 1 o (SZMN), Khachmas Distr., nr. Mukhtadir Vil., 30.06.1977, PD.

COMMENTS. An eastern Mediterranean species, known from Italy and Libya [Wesołowska, 1986: map 901], throughout north Africa, Greece, Croatia [Metzner, 1999; Logunov, Chatzaki, 2003] and the Near East [e.g., Karol, 1967; Prószyński, 2003; Uyar, Uğurtaş, 2012; Coşar et al., 2014], to central Iran [Logunov et al., 2002] and Azerbaijan [Rakov, Logunov, 1996; Marusik et al., 2004: sub H. equestior].

Heliophanus kochii Simon, 1868

MATERIAL. PORTUGAL: 2 우 (OUMNH), Lisbon, "bottle 216122 - - SPAIN: 1 (NMPC), Barcelona, 25.04.2004, LD; 3 $\sigma^{T} \sigma^{T}$ (NMPC), Xávia (=Jávea), NP del Mongú, 29.04.2004, LD; 2 $\mathrm{O}^{7} \sigma^{7}$ (NMPC), Besalú, 2.05.2004, LD; $5 \mathrm{O}^{7} \sigma^{7}, 1$ ( 1 (NMPC), Catalonia, Mataró (c. $\left.41^{\circ} 32^{\prime} \mathrm{N}, 2^{\circ} 27^{\prime} \mathrm{E}\right), 1.05 .2004$, LD. - ITALY: 5 우 (SMFM), Sardinia, no exact locality and collector name; $10^{7}$ (SMFM), S Italy, Monte Garyano, 09.1951, coll.? — CROATIA: 1 $\mathrm{O}^{7}$ (SMFM), Trogiv, c. $20 \mathrm{~km} \mathrm{~N}$ of Split, 18.04.1956, O. Kraus. GREECE: $10^{7}, 1$ (SMFM), Island Kos, nr. Zia, 500-800 m a.s.l., forest and macchia, 29.04.1968, JMS; 1 क (SMFM), Island Kos, Kastell, 28.04.1968, JMS; $10^{7}$ (LMNM), Lesbos, nr. Potamia (39 $\left.13^{\prime} 03^{\prime \prime} \mathrm{N}, 26^{\circ} 09^{\prime} 48^{\prime \prime} \mathrm{E}\right), 23.04 .1997, \mathrm{CF} ; 1$ \% (LMNM), Lesbos, Stavros $\left(39^{\circ} 02^{\prime} 12^{\prime \prime} \mathrm{N}, 26^{\circ} 16^{\prime} 45^{\prime \prime} \mathrm{E}\right)$, c. $158 \mathrm{~m}$ a.s.1., damp, rocky and grazed roadside field with low shrubs among grass, 21.04.1997, $\mathrm{SJ} ; 1$ (LMNM), Lesbos, Skala Kallonis $\left(39^{\circ} 12^{\prime} 25^{\prime \prime} \mathrm{N}, 26^{\circ} 13^{\prime} 13^{\prime \prime} \mathrm{E}\right)$, back of beach and along river bank, 23.04.1997, SJ; $10^{7}, 1$ 오 (PCRG), Thasos, Ipsarion $\left(40^{\circ} 39^{\prime} \mathrm{N}, 24^{\circ} 40^{\prime} \mathrm{E}\right), 820 \mathrm{~m}$ a.s.1., 27.05 . 2003, AF; 1 \& (SMFM, 5152), Saloniki, no date and collector name; $1 \sigma^{7}$ (MHNG), Nafpaktos, Etoile-Acarnanie, 14.04.1979, S. Vit. - TURKEY: $1 \mathrm{O}^{7}$ (PCRS), meadow c. $1 \mathrm{~km} \mathrm{~W}$ of Yalikavak, nr. Bodrum, under stone, 28.05.1997, ARS; 1 \& (LMNM), Içel, Tekmen, terraced cultivated slopes above sea, 8.06.1993, CF; 1 ㅇ (LMNM), Içel, Çamlıyayla, Dağ Hotel area $\left(37^{\circ} 10^{\prime} 10^{\prime \prime} \mathrm{N}, 34^{\circ}\right.$ $\left.36^{\prime} 02^{\prime \prime} \mathrm{E}\right), 1454 \mathrm{~m}$ a.s.l., limestone cliff, 1.07.1995, SJ \& CF; 2 우우 (LMNM), Içel, Narlıkuyu ( $\left.36^{\circ} 26^{\prime} 0422 \mathrm{~N}, 34^{\circ} 06^{\prime} 44^{\prime \prime} \mathrm{E}\right)$, garrigue vegetated limestone dominated by Pinus, 5.06.1994, MB; 1 우 (LMNM), Içel, c. $2 \mathrm{~km} \mathrm{~S}$ of Camlidere (36 $\left.52^{\prime} 12^{\prime \prime} \mathrm{N}, 34^{\circ} 24^{\prime} 37^{\prime \prime} \mathrm{E}\right)$, rocky disused quarry at side of road, dominated by Euphorbia, 
Mullein Cistus and Rubus, 30.06.1995, SJ \& CF; 4 +o (ZMUM) Antalya, Kemer, pine forest, 50-100 m a.s.1., 23-28.04.2001, KM; 2 우 (ZMTU), Izmir Distr., Yamanlar Daği, unders stones on a dry rocky slope, 25.05.1973, PL; $3 \sigma^{7} \sigma^{7}, 3$ 우 (ZMTU), same locality, under stones in the alpine zone (ca $1100 \mathrm{~m}$ a.s.1.), 24.05.1973, PL \& F. Önder; $1 \sigma^{7}, 4$ ㅇ (PCSD), nr. Fethiye, above Arpacik, 28.05. 1997, RS; 1 (NHMW), Hereke, c. 23 km W of Ižmit, 50-100 m a.s.1., olive plantation (under stones and in grass), 25.05.1967, JG, FR \& AR; $1 \sigma^{7}$ (NHMW), c. $12 \mathrm{~km} \mathrm{SSW} \mathrm{of} \mathrm{Adapazarı,} \mathrm{c.} 100 \mathrm{~m}$ a.s.1., oak forest with Carpinus, Tilia, Ligustrum, Crataegus, etc., 14.06.1967, JG, FR \& AR; 1 क (NHMW), Hisarcık, SE Kayseri, 1500-1600 $\mathrm{m}$ a.s.1., sparce oak forest, 7.06.1967, JG, FR \& AR; 19 (NHMW), Pozante, river valley and on slopes with Pinus sp. and shrubs, 8.05.1969, AR; 1 フ (NHMW), Akşehir, Sültandağ, 24.04 1960, FR; $1 \sigma^{7}$ (NHMW), Namrun, pine forest, 12.05-5.06.1964, FR; $1 \sigma^{7}$ (NHMW), Namrun, S-slope of Taurus, c. $1200 \mathrm{~m}$ a.s.1., 911.05.1969, AR; $1 \sigma^{7}$ (NHMW), Pinus halepensis forest, 9.06.1977, H. Nemenz; 1 (SMFM), Manyas Gölü, E lake shore nr. Kuşcenneti, $\mathrm{S}$ of national park, no date, coll.? - SYRIA: $8 \sigma^{\top} \sigma^{\top}, 3$ + 0 (NMPC) NW Syria, Mashtal Helu nr. Homs, 30.03-3.04.2001, MR; 1 O (NMPC), NW Syria, Aln al Zara nr. Idlib, canyon of Oronthes, 28.03.2001, MR; 1 \% (NMPC), NW Syria, castle Qalaat al Hosn nr. Homs, 3-4.04.2001, MR; 1 क (NMPC), NW Syria, Al Haffeh nr. Latakia, 13.04.2001, MR

COMMENTS. A European - Mediterranean species, reaching the Crimea in the north-east [Kovblyuk, 2004] and also recorded from Asia Minor and Levant [Kulczyński, 1903; Wesołowska, 1986; Metzner, 1999; Prószyński, 2003; Logunov, 2012; Helsdingen, 2013; Ijland, Helsdingen, 2014; Coşar et al., 2014]. New record for Syria.

\section{Heliophanus lineiventris Simon, 1868}

MATERIAL. SPAIN: $1 \sigma^{7}$ (NMPC), Catalonia, Mataró (c. $\left.41^{\circ} 32^{\prime} \mathrm{N}, 2^{\circ} 27^{\prime} \mathrm{E}\right), 1.05 .2004$, LD. - ITALY: $1 \sigma^{\top}$ (SMFM), Sicily, E of Ficuzza, edge of a path, thorny, dry (under large stones), c $900 \mathrm{~m}$ a.s.1., 10.04.1957, HK. - ALBANIA: 1 오 (SMFM, 1987), 'Korab', no date and collector. - GREECE: $1 \sigma^{7}$ (SMFM), Korfu nr. Limni Antiniotissa $\left(39^{\circ} 46^{\prime} 57.3^{\prime \prime} \mathrm{N}, 19^{\circ} 51^{\prime} 34.8^{\prime \prime} \mathrm{E}\right)$, at beach $(0$ $\mathrm{m}$ a.s.1.), Macchina and meadows (under stones and wood), 23.03. 2006, AS; $1 \sigma^{7}$ (SMFM), Thrakien, Porto Lago, Ewru, 1.04.1977, RK. - TURKEY: 1 ( NMPC), Sivas Prov., nr. Demiryurt, Tödürge Gölü (lake), c. $13 \mathrm{~km} \mathrm{~W}$ of Zara (39 $\left.53^{\circ} \mathrm{N}, 3^{\circ} 37^{\prime} \mathrm{E}\right), 25-28.06 .2002$, MR; 1 9 (NMPC), Cağlayan, c. $24 \mathrm{~km} \mathrm{SE}$ of Erzincan $\left(39^{\circ} 36^{\prime} \mathrm{N}\right.$ $39^{\circ} 42^{\prime} \mathrm{E}$ ), valley above waterfall, 23-24.06.2002, MR; 1 ㅇ (NHMW), Burdur, 4.05.1965, FR.

COMMENTS. A trans-Eurasian temperate species [Logunov, Marusik, 2000b].

\section{Heliophanus machaerodus Simon, 1909}

MATERIAL. MOROCCO: 1 (ZMUM), Ketama Prov., c. 40 $\mathrm{km}$ W of Ketama, along P39, c. $1030 \mathrm{~m}$ a.s.l., among stones and grasses in short vegetation in degraded Quercus forest, 20.04.1984, RB. - ALGERIA: $1 \sigma^{7}$ (PCRB), Wilaya Blida, Atlas Blidéen, Djebel Mouzaia, c. $1250 \mathrm{~m}$ a.s.l., under stones along the lake, 14.05.1988, RB; 1 o (PCRB), Wilaya Bejaia, E of the mouth of Wadi Daas, c. $5 \mathrm{~m}$ a.s.1., under stones and on bushes on the shore, 22.05.1988, RB; 2 +o (PCRB), Wilaya Saida, Mts Daia, forestry headquater in Merdja, c. $750 \mathrm{~m}$ a.s.1., among stones in open, dry Pinus halepensis forest, 4.05.1984, RB; 1 क (PCRB), Wilaya Bejaia, S. Tichi, c. $50 \mathrm{~m}$ a.s.1., under shrubs along Wadi Djemaa, 20.05. 1988, RB; $1 \sigma^{7}$ (PCRB), Wilaya Aïn Defla, N. Derrag, forest Aghbal, c. 1020 m a.s.1., under stones, 23.04.1989, RB. - TUNISIA: $1 \sigma^{7}, 1+$ (SMFM), Tunis, Karthago, right side of the entry to Roman thermes, dense Mesembrianthemum, humus soil, mostly in funnel, 27.03.1959, HK.

COMMENTS. Recorded from Morocco, Algeria and Tunisia only [Wesołowska, 1986; present data]. New record for Tunisia.
Heliophanus melinus L. Koch, 1867

MATERIAL. GREECE: $1 \sigma^{7}$ (SMFM), Kelempek Mts, above Neda, 400-500 m a.s.1., Quercus pubescens, 4-7.05.1968, O.V Helversen \& AS; $1 \sigma^{\top}$ (LMNM), Chios, Giosonas Beach (38 $33^{\prime}$ $\left.32^{\prime \prime} \mathrm{N}, 26^{\circ} 04^{\prime} 53^{\prime \prime} \mathrm{E}\right)$, scrub and herbs on stabilized shore, 28.04.1997, CF; 1 ㅇ (PCRG), Thasos, Skala Kallirachis $\left(40^{\circ} 39^{\prime} \mathrm{N}, 2^{\circ} 40^{\prime} \mathrm{E}\right)$, c. $2 \mathrm{~m}$ a.s.1., 27.05.2003, AF; $1 \sigma^{7}$ (SMFM), 'Aegina', no exact locality and date; 1 + (SMFM), 'Ateropolis', no exact locality and date; 1 ( (SMFM), Crete, Topolia, 06.1926, CR; 1 ㅇ (SMFM), Chalkidiki, Petralona, 20.08.1971, RK; 2 우 (SMFM), Crete, SW Ajil deka, edge of Messara valley, sands with Medicago lupulina, 10.04.1958, HK; $1 \sigma^{7}$ (SMFM), Crete, c. 3 km SE of Kaluthiana, $\mathrm{W}$-slope, cultivated land with ruin and stonewalls, Olea europea, Ouercus ruber, low grass (under vstones), c. $50 \mathrm{~m}$ a.s.1., 19.04.1958, HK; 1 O (SMFM), Crete, valley NW of Lake Curna, rocky, dense bushes, Ficus, Quercus coccifera, on stones, 15.04.1958, HK; $1 \sigma^{7}$ (SMFM), Crete, nr. Ajil Deka, Gortys, W-slope, 100-120 m a.s.1., rocky, indet. yellow flowers, Cirsium, Cystus, 9.04.1958, HK; 2 $\sigma^{7} \sigma^{7}$ (SMFM), same locality, 85-100 m a.s.1., stony, grass, Cirsium, 9.04.1958, HK; 2 ○ $\sigma^{7}$ (SMFM), Crete, S-side of Lake Curna, slope scree, Cirsium, reed, yellow Laminacea, 15.04.1958, HK; 1 or (MHNG), Crete, Lassithi, Sitia, peninsular Faneromeni, a 12 ouest de la localite, c. $5 \mathrm{~m}$ a.s.1., 6.05.1985, BH; $1 \sigma^{7}$ (MHNG), Nafpaktos, Etoile-Acarnanie, 14.04.1979, S. Vit; 2 ○ $^{7}$ (MHNG), Naxos, pres de Galini, le long des cheminis campestres, c. $25 \mathrm{~m}$ a.s.1., 25.04.1987, BH; 1 ○ (SMFM), Kelempek Mts, summit region, c. $900 \mathrm{~m}$ a.s.1., Quercus pubescens forest, 8.05.1968, JMS. - TURKEY: 19 (PCRS), Akyaka, nr. Cinan Beach, under stones in pine wood, 30.05.1996, ARS; $4 \sigma^{7} \sigma^{7}$ (NMPC), Sivas Prov., Nr. Demiryurt, Tödürge Gölü (lake) (c. $13 \mathrm{~km} \mathrm{~W}$ of Zara; $39^{\circ} 53^{\prime} \mathrm{N}$, $\left.37^{\circ} 37^{\prime} \mathrm{E}\right), 25-28.06 .2002, \mathrm{MR} ; 2 \sigma^{7} \sigma^{7}, 13$ 우 (ZMTU), Izmir Distr., Yamanlar Daği, unders stones on dry rocky slope, 25.05.1973, PL, F. Önder \& E. Pehlivan; $2 \sigma^{7} \sigma^{7}, 1$ (ZMTU), same locality, under stones in the alpine zone (c. $1100 \mathrm{~m}$ a.s.1.), 24.05.1973, PL \& F. Önder; 1 ( (PCSD), Pamukkale, ruins of Heiropolis, 26.05.1997,

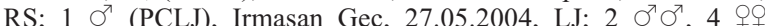
(MMUM), Adana Prov., Pozant1 Distr., Belemidik Vil., 18.05.2003, KK; $3 \bigcirc^{7} \sigma^{7}, 7$ 9 (MMUM), Adana Prov., Belemedik Vil., 18.05. 2003, KK; 1 क (MMUM), Adana Prov., Pozantı Distr., Belemedik Vil., 7.06.2002, KK; 1 q (MMUM), Mersin Prov., Gülek Pass, 89.07.2002, KK; 1 9 (MMUM), Niğde Prov., Ulukışla Distr., Kolsuz Pass, 14.05.1996, KK; 2 क् (LMNM), Adana, c. $8 \mathrm{~km} \mathrm{~S}$ of Sambeyli, 13.07.1992, SJ; 1 † (LMNM), İsparta, Kovada Gölü (lake), sparsely vegetated lakeside shingle, 20.06.1993, CF; 2 우 (LMNM), Konya, Sultan Dağlari, c. 7 km NE of Bâgkonak, 8.07. 1992, CF; 1 O (LMNM), Bağkonak, Sultan Dağlari, Konya, under dwarf hillside herbs, 19.09.1993, CF; 1 q (LMNM), Içel, c. $31 \mathrm{~km} \mathrm{~N}$ of Anamur $\left(36^{\circ} 12^{\prime} 11^{\prime \prime} \mathrm{N}, 32^{\circ} 54^{\prime} 01^{\prime \prime} \mathrm{E}\right)$, young, goat-grazed, pine plantation on high, rock strewn, mountain slope, 7.05.1994, SJ \& CF

COMMENTS. A European - Mediterranean species, known from Spain [Helsdingen, 2014] to Asia Minor [Kulczyński, 1903; Uyar, Uğurtaş, 2012; Helsdingen, 2013; Coşar et al., 2014]; according to Wesołowska [1996], the records of $H$. melinus from the Caucasus and Central Asia are likely to belong to H. patagiatus Thorell, 1875.

Heliophanus mordax (O. Pickard-Cambridge, 1872)

MATERIAL. GREECE: $1 \sigma^{7}$ (MHNG), Island of Rhodes, montagne Profitis Ilias, c. $650 \mathrm{~m}$ a.s.1., 15.05.2001, CL. - CYPRUS: 1 $\sigma^{7}$ (PCRS), nr. Souskiok, under stones in grassland, 1.05.2013, coll.?; 1 ( $\mathrm{PCDM}$ ), house in Dali, in garden, spring 2004, DMC; $1 \sigma^{7}$ (PCDM), Agridi, house yard, May 2012, DMC. - TURKEY: 2 90 (PCRS), Mazi Beach, nr. Bodrum, under stone in field, 31.05. 1996, ARS; 1 O7, 1 † (LMNM), Nevşsehir, Nevşehir-Urgur, 11.07. 1992, CF; 1 ○ (LMNM), Kayseri, Tahtali Daglari, c. 8 km E of Bakirdagi, 13.07.1992, CF; 1 o (LMNM), Kayseri, c. $31 \mathrm{~km} \mathrm{SE} \mathrm{of}$ Develi $\left(38^{\circ} 15^{\prime} 02^{\prime \prime} \mathrm{N}, 35^{\circ} 45^{\prime} 27^{\prime \prime} \mathrm{E}\right), 12.05 .1994$, SJ \& CF; $1 \sigma^{7}$ (LMNM), Nevşehir-Ürgüp, Nevşehir, calcareous herb-rich grassland, 16.06.1993, CF; 1 \% (LMNM), Bağkonak, Sultan Dağlari, Konya, under dwarf hillside herbs, 19.09.1993, CF; 1 o (LMNM), 
Konya, Tuz Gölü (lake), Yavşan Tuslası ( $\left.38^{\circ} 45^{\prime} 43^{\prime \prime} \mathrm{N}, 33^{\circ} 09^{\prime} 31^{\prime \prime} \mathrm{E}\right)$, stony railway embankment with sparse halophytic vegetation adjacent to evaporating lagoon, 18.06.1993, CF; $1 \sigma^{7}$ (LMNM), Niğde $\left(38^{\circ} 19^{\prime} 37^{\prime \prime} \mathrm{N}, 34^{\circ} 14^{\prime} 34^{\prime \prime} \mathrm{E}\right)$, c. $33 \mathrm{~km}$ E of Aksaray, Selime Bridge, disturbed river bank and herb-rich limestone cliff base, 10.05.1994, SJ \& CF; $1 \sigma^{7}$ (LMNM), Niğde, c. 8 km W of Gözlükuyu, c. 1 km along Ilhara road off Askeray-Adana road, herb-rich, very rocky abandoned farmland edge, 10.05.1994, SJ \& CF; $1 \mathrm{\sigma}^{7}$ (LMNM), Zonguldak, Amasra, waste ground by flats, 18.05.1994, SJ \& CF; 1 + (LMNM), Kayseri, c. $3 \mathrm{~km} \mathrm{~N}$ of Saraycik $\left(38^{\circ} 11^{\prime} 19^{\prime \prime} \mathrm{N}, 35^{\circ}\right.$ 55'33"E), 4.07.1995, SJ \& CF; 1 ( (OUMNH), Nevsehir, Kessehis Gulsehir, under stone, 3.08.1956, GL; 1 오 (NMPC), Tunceli (39 $\left.06^{\prime} \mathrm{N}, 39^{\circ} 33^{\prime} \mathrm{E}\right)$, Munsur river, 22.06.2002, MR; $1 \mathrm{O}^{\top}, 1$ ㅇ (PCSD), nr. Fethiye, above Arpacik, 28.05.1997, RS; 1 ๆ , 1 ( $($ NHMW), Eskigümüs, NE of Niğde, $1400-1500 \mathrm{~m}$ a.s.l., steppe on the edge of orchard, 9.06.1967, JG, FR \& AR; $2 \sigma^{7} \sigma^{7}, 1$ (NHMW), Akşehir, Sültandağ, 5-27.05.1960, FR; 1 + (NHMW), Namrun, pine forest, 30.05.1964, FR. - AZERBAIJAN: 1 o (ZMUM), Turianchai, 18.05.1983, PD; 1 (ZMUM), c. $50 \mathrm{~W}$ of Baku, nr. Dzheirankechmez, 2.07.1988, PD; 1 (SZMN), Astara Distr. between Motlayatat and Bilyasar, Talysh Mts $\left(38^{\circ} 36^{\prime} \mathrm{N}, 48^{\circ} 40^{\prime} \mathrm{E}\right)$, 600-700 m a.s.1., Carpinus-Acer-Quercus forest, 20-22.06.2003, AC. - IRAN: $2 \sigma^{\top} \sigma^{7}$ (MMUM), Zaghe, 13.10.2011, SZ.

COMMENTS. An eastern Mediterranean - Central Asian subboreal species, known from Dodecanese islands and Egypt [Metzner, 1999], throughout Asia Minor and the Near East [Prószyński, 2003; Topçu et al., 2006; Coşar et al., 2014], to the Caucasus, eastward to SW Kopetdagh in Turkmenistan [Rakov, Logunov, 1996; Marusik et al., 2004] and Khorasan province of Iran [Logunov et al., 2013]. New record for Cyprus.

Heliophanus patagiatus Thorell, 1875

MATERIAL. ARMENIA: 6 우 (ZISP), Razdansky Distr., nr. Ankavan, Marmarik river, 13.08.1979, V.P. Tyshchenko.

COMMENTS. A trans-Eurasian temperate species [Logunov, Marusik, 2000b].

Heliophanus simplex Simon, 1868

MATERIAL. AUSTRIA: $1 \sigma^{7}$ (OUMNH), Tyrol, Cortina g’Ampezz, 1900, G. Nicholson. - ALBANIA: 1 (SMFM, 1994) Kula Lums (=Kula Ljums), 4.07.1918, coll.? — TURKEY: 2 o

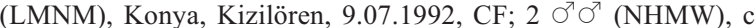
$20 \mathrm{~km} \mathrm{~N}$ of Turhal, $800-1000 \mathrm{~m}$ a.s.l., pine forest, 4.06.1967, JG.

COMMENTS. A central European - eastern Mediterranean species, eastward to Asia Minor and the Crimea [Metzner, 1999; Russell-Smith et al., 2011; Kovblyuk, 2004; present data].

\section{Heliophanus stylifer Simon, 1878 \\ Figs $31-33,37-39$.}

MATERIAL. ALGERIA: $1 \sigma^{7}$ (PCRB), Wilaya Blida, Meftah, Djebel Zerouela, c. $450 \mathrm{~m}$ a.s.1., among stones and Graminea in maquis, 23.05.1985, RB; 1 (PCRB), Wilaya Chleff, Damous, c. 5 $\mathrm{m}$ a.s.1., litter on the shore, 8.05.1986, RB; 1 \% (PCRB), Wilaya El Tarf, W of El Kala, S of Bou Malek, Lac Melah, c. $3 \mathrm{~m}$ a.s.1., in litter of Quercus suber forest (very close to the lake), 6.04.1982, RB; $1 \mathrm{O}^{7}, 1$ क (ZMUM), Wilaya Bejaia, Tichi, c. $10 \mathrm{~m}$ a.s.1. wasteland in dunes, in Acacia litter, 21.05.1988, RB; $1 \sigma^{7}, 2$ 우 (MMUM), Wilaya Bejaia, S of Tichi, c. $50 \mathrm{~m}$ a.s.l., under shrubs along Wadi Djemaa, 20.05.1988, RB.

COMMENTS. Recorded from Morocco and Algeria only [Wesołowska, 1986; present data].

This species is very similar to $H$. ramosus; the main diagnostic characters for both sexes between two species are given above under the 'Diagnosis' of H. ramosus.
Heliophanus tribulosus Simon, 1868

MATERIAL. CROATIA: $1 \sigma^{7}$ (SMFM, 7701), Dalmatia, 05.1938, coll.? - GREECE: 1 9 (PCRG), Profitis Ilias, 1.10.2001, coll.?; 1 \% (PCRG), Thasos, Thasos valley plain woodland (c. $40^{\circ}$ $\left.39^{\prime} \mathrm{N}, 24^{\circ} 40^{\prime} \mathrm{E}\right), 120 \mathrm{~m}$ a.s.1., 25.05.2003, AF; $10^{\top}$ (SMFM), S coast of Peloponnes, Ligia, beach, dunes and adjacent cultural landscape, 1.06.1998, LM. - TURKEY: 1 \% (LMNM), Konya, c. $10 \mathrm{~km}$ along Akșehir-Cetince road, Sultan Dağları, mixed herbs and shrubs among young pine plantation, 19.06.1993, SJ \& CF; 1 $\sigma^{7}$ (LMNM), Içel, Narlıkuyu (36 $\left.26^{\prime} 04^{\prime \prime} \mathrm{N}, 34^{\circ} 06^{\prime} 44^{\prime \prime} \mathrm{E}\right)$, garrigue vegetated limestone dominated by Pinus, 5.06.1994, MB; 1 \% (ZMTU), Izmir Distr., Yamanlar Daği, on wet ground along a mountain brook, 24.05.1973, PL; $10^{7}$ (NHMW), c. $80 \mathrm{~km} \mathrm{SW}$ of Kale, the road Denizli-Muğla, 22.05.1969, AR.

COMMENTS. A European - Mediterranean species, known France [Helsdingen, 2014] to Asia Minor [Kulczyński, 1903; Uyar, Uğurtaş, 2012; Helsdingen, 2013; Coşar et al., 2014; present data]. Some of the earlier records of $H$. tribulosus from the Iberian Peninsula [Wesołowska, 1986; Helsdingen, 2014] actually belong to the closely related $H$. haymozi sp.n. (see above).

'Hyllus' insularis Metzner, 1999

MATERIAL. TURKEY: 1 ㅇ (PCRS), Akyaka, Sulemin's castle, under stones, 28.05.1996, ARS; 1 क (PCLJ), Nemrut Dağ, on stony ground, 1.06.2004, LJ; 2 O $^{\top} \mathrm{O}^{\top}$ (NHMW), Kaș, 23-24.04.1964, FR. - IRAN: $2 \sigma^{7} \sigma^{7}$ (MMUM), Lorestan Prov., Khorramabad (c. $\left.33^{\circ} 29^{\prime} \mathrm{N}, 48^{\circ} 21^{\prime} \mathrm{E}\right), 16.09-9.11 .2011, \mathrm{SZ}$.

COMMENTS. Recorded from Lesbos Island, Turkey and Iran (Tehran, Yazd and Mazandaran provinces) [Metzner, 1999; Logunov, 2001b; present data]. New record for Turkey.

Icius congener (C.L. Koch, 1846)

MATERIAL. ALGERIA: $1 \sigma^{7}$ (PCRB), Wilaya Tizi Ouzou, Massif Djurdjura, Tala Guilef, c. 2000 m a.s.1., mountain grassland, 2.05.1985, RB.

COMMENTS. A west-Mediterranean species, known from the Iberian Peninsula and Algeria to Italy (mainland) [Alicata, Cantarella, 1994; Helsdingen, 2014; present data].

It is very likely that Alicata \& Cantarella [1994] mismatched the male and female of $I$. congener, as they had no sample containing both sexes collected together. They studied the sample 25700 from the Natural History Museum in Paris which contained 51 females identified by E. Simon himself as I. congener. However, as Alicata \& Cantarella [1994: 120] stated only two of these females really belonged to I. congener, the rest were those of I. hamatus. Besides, no male was available in this sample. As a result, these authors seemed to have illustrated and described two untypical females of I. hamatus and misidentified them for those of $I$. congener. In my opinion, the true female of $I$. congener remains unknown yet.

\section{Icius hamatus (C.L. Koch, 1846)}

MATERIAL. ALGERIA: 1 r", 2 우 (PCRB), Wilaya Blida, Atlas Blidéen, Djebel Mouzaia, c. $1250 \mathrm{~m}$ a.s.1., under stones along the lake, 14.05.1988, RB; $1 \sigma^{7}$ (PCRB), same locality, in litter of Quercus faginea forest, 6.06.1986, RB; $1 \sigma^{7}$ (PCRB), Wilaya Blida, Atlas Blidéen, Chrea, c. $1100 \mathrm{~m}$ a.s.l., pitfall traps in Quercus ilex forest, 20.06.1987, RB; 1 \% (PCRB), same locality, c. $1045 \mathrm{~m}$ a.s.1., pitfall traps in dense Quercus ilex forest, 15.02- 
21.06.1987, RB; 1 ㅇ (PCRB), Wilaya Tizi Ouzou, Massif Djurdjura, Tala Guilef, c. $1500 \mathrm{~m}$ a.s.1., pitfall traps in Quercus ilex forest 25.11.1986-29.05.1987, RB; 1 \% (PCRB), Wilaya Alger, Beaulieu, c. $50 \mathrm{~m}$ a.s.1., in garden, no date, RB; $1 \sigma^{7}, 1$ (PCRB), Wilaya Aïn-Defla, Djebel Zaccar, S. Aïn N'Sour, c. 900 m a.s.1., pitfall traps and litter in Quercus ilex forest, 18.05.1988, RB; 1 \%, 1 (ZMUM), Wilaya Oran, Hadjadz, c. $50 \mathrm{~m}$ a.s.1., in garden, 0708.1988, RB; 2 O $^{\top}, 3$ 0 + (SZMN), Wilaya Bejaia, S. Tichi, c. 50 $\mathrm{m}$ a.s.1., under shrubs along Wadi Djemaa, 20.05.1988, RB; 1 (PCRB), Wilaya Alger, Beaulieu, c. $50 \mathrm{~m}$ a.s.l., in garden, no date, RB; $1 O^{7}$ (PCRB), Wilaya El Tarf, El Kala, Lac Oubeira (N-side), S of Djebel Bou Malek, c. $3 \mathrm{~m}$ a.s.1., in litter nr. the lake, 3.04.1982, RB; 1 q (PCRB), Wilaya Aïn Defla, Wadi Djer, c. 240 m a.s.1., nr. water, 4.05.1989, RB; 2 +o (PCRB), Wilaya Ech Cheliff, S of Tenes, gorges of Wadi Allata, c. $125 \mathrm{~m}$ a.s.1., under stones, 6.05. 1989, RB; 1 (PCRB), Wilaya El Tarf, W of El Kala, S of Bou Malek, Lac Melah, c. $3 \mathrm{~m}$ a.s.1., in litter of Quercus suber fores (very close to the lake), 6.04.1982, RB. - PORTUGAL: $5 \sigma^{7} \sigma^{7}, 4$ qo $(\mathrm{FMNH})$, Mértola (c. $\left.37^{\circ} 38^{\prime} \mathrm{N},-7^{\circ} 40^{\prime} \mathrm{W}\right), 24.05 .2003, \mathrm{PC} ; 5$ $\sigma^{7} \sigma^{\top}, 8$ 우 (FMNH) \& $2 \sigma^{7} \sigma^{7}, 2$ 우 (ZMUM), Paúl do Boquilobo Biosphere Reserve (c. $\left.39^{\circ} 23^{\prime} \mathrm{N},-8^{\circ} 32^{\prime} \mathrm{W}\right), 9.05-2.08 .2002, \mathrm{PC}$. SPAIN: $2 \sigma^{\top} \sigma^{7}$ (MVHN), Valencia, Devesa del Saler (Estany de Pujol / Dunas Fijas), 18.05-28.06.2004, DD; 2 우 (SMFM), La Rabida, 05.1959, J. Büddenbrock; 2 + (NMPC), Guardamar del Segura, 28.04.2004, LD; $3 \sigma^{7} \sigma^{7}, 5$ 50 (NMPC), the nature reserve of La Albufera, S of the city of Valencia, 30.04.2004, LD; $16 \sigma^{7} \sigma^{7}$, 9 우 (NMPC), Valensia, Guadalest (c. $38^{\circ} 41^{\prime} \mathrm{N}, 0^{\circ} 11^{\prime} \mathrm{E}$ ), 27.04.2004, LD; 1 $\sigma^{7}$ (NMPC), Xávia (=Jávea), NP del Mongú, 29.04.2004, LD; 3 ○ $\sigma^{7}$ (NMPC), Besalú, 2.05.2004, LD; 1 б (NMPC), Salou, 26.04.2004, LD. - ITALY: 2 + $($ (OUMNH), St Margarita, stones, 20.08.1958, GL. - TUNISIA: 1 ㅇ (SMFM), NE Tindja, olive grove, dense grass with Lycnis viscaria, Cirisium and Asphodelus (under stones and in litter), 9.04.1959, HK. - GREECE: 1 (SMFM), Crete, c. $8 \mathrm{~km} \mathrm{~W}$ of Chania, camp at reservoir $\mathrm{nr}$. Ajia, meadow with Aspidium, Cirsium, Citrus sinesis and Platanus, 22-24.04.1958, HK

COMMENTS. A European - Mediterranean species, known from the Iberian Peninsula to Asia Minor [Helsdingen, 2014], including the records from northern Africa (Tunisia) [Bosmans, 2003; present data]. The records of I. hamatus from China (Tibet; see Song et al. [1999]) were likely to be based on a misidentification.

Icius simoni Alicata et Cantarella, 1994

MATERIAL. ALGERIA: $1 \sigma^{7}$ (PCRB), Wilaya Maghnia, Tafna, along Wadi Tafna, c. $350 \mathrm{~m}$ a.s.l., among stones close to water and among Oxalis, 23.04.1984, RB.

COMMENTS. The species is known from Algeria only [Alicata, Cantarella, 1994; present data].

Icius subinermis Simon, 1937

MATERIAL. PORTUGAL: $20^{7} \sigma^{7}, 2$ 오 (FMNH), Paúl do Boquilobo Biosphere Reserve (c. $39^{\circ} 23^{\prime} \mathrm{N},-8^{\circ} 32^{\prime} \mathrm{W}$ ), 28.072.09.2002, PC

COMMENTS. A west-Mediterrannean - European species, known from the Iberian Penisula to Germany and Macedonia [Alicata, Cantarella, 1994; Jäger, 1995; Helsdingen, 2014]

The species Icius lamellatus Wunderlich, 2011 described by Wunderlich [2011] from Italy and Portugal seems to be a junior synonym of I. subinermis; see also 'Comments' above under I. insolitus.

Leptorchestes peresi (Simon, 1868)

MATERIAL. ALGERIA: $10^{7}$ (PCRB), Wilaya Medea, Col de Beni Chicao, c. $1230 \mathrm{~m}$ a.s.l., traps in mixed forest of Quercus ilex and Q. suber, 13.01.1989-20.05.1990, RB; $1 \sigma^{7}$ (PCRB), Wilaya
Tlemsen, SE of Tlemsen, forest d'Hafir, c. $1350 \mathrm{~m}$ a.s.l., mixed forest of Quercus suber, Q. ilex, Juniperus sp. and Erica arborea, sweeping in vegetation, 6.05.1984, RB; $1 \mathrm{O}^{7}$ (PCRB), Wilaya Tipasa, Douaouda, Wadi Mazafran, $50 \mathrm{~m}$ a.s.1., glades in forest of Populus alba, 16.12.1986-26.02.1988, RB; 1 O', 1 ㅇ (ZMUM), Wilaya Tipasa, Sidi Fredj, c. $10 \mathrm{~m}$ a.s.l., pitfall traps in Olea stands, 10.12.1986-20.12.1987, RB. - PORTUGAL: $1 \mathrm{O}^{7}$ (FMNH), Mértola (c. $\left.37^{\circ} 38^{\prime} \mathrm{N},-7^{\circ} 40^{\prime} \mathrm{W}\right), 28.05 .2003$, PC. - SPAIN: $10^{\prime \prime}$ (MMUM, G7512.1669), Sinues, Jaca, in pine wood, 22.06.1972, ED; 4 우 (MMUM, G7512.1666), San Juan de la Pena, Huesca, on northwest-facing rock slope, 2.06.1975, ED.

COMMENTS. A west-Mediterranean species, known from Algeria [present data] and the Iberian Peninsula, throughout south France to Greece (mainland) [Metzner, 1999; Wesołowska, Szeremeta, 2001; Ijland, Helsdingen, 2014]. New record for Algeria.

\section{Macaroeris flavicomis (Simon, 1884)}

MATERIAL. MATERIAL. FRANCE: $3 \sigma^{7} \sigma^{7}, 2$ Oᄋ (PCJM, 14818), Lot, Garonne, Boudy, c. 150 m a.s.l., scrube, oak woods, 7.08.1985, JFM; 1 O' (PCJM, 20493), Morbihan, Le Passage, c. 10 m a.s.1., march, scrube, pines, 26.05.1992, JFM; 1 (PCJM, 20442), Morbihan, Bailleron, c. $10 \mathrm{~m}$ a.s.l., stones \& shrubs, 24.05.1992, JFM; 4 O $^{7} \sigma^{7}$ (PCJM, 11632), Lot, Gignac, c. $300 \mathrm{~m}$ a.s.l., stony oak scrube, 13.05.1984, JFM; 1 O (PCJM, 11681), Lot, Cales, c. 300 $\mathrm{m}$ a.s.1., stony juniper scrub, 14.05.1984, JFM; 2 O $^{\top} \sigma^{\top}, 1$ ㅇ (PCJM, 11720), Lot, Bernades, c. $300 \mathrm{~m}$ a.s.l., stony oak scrub, 15.05.1984, JFM; 3 O $^{7} \mathrm{O}^{7}$ (PCJM, 11777), Aveyron, Vezac, c. $300 \mathrm{~m}$ a.s.l., box hedge (litter), 20.05.1984, JFM; $20^{7} 0^{7}$ (PCJM, 11859), Aveyron, Floirac, c. $300 \mathrm{~m}$ a.s.1., stony oak scrub, 23.05.1984, JFM; 1 o (PCMJ), Pyrénées-Orientales, Col d'Quillat, 26-27.05.1987, PP; 1 O (PCMJ), Pyrénées-Orientales, Maureillas, 22-31.05.1987, PP; 1 † (PCMJ), Var, Méounes les M., 21.03-2.06.1989, PP; 1 \% (PCMJ), Gard, Quissac, 19-29.10.1989, PP; 1 + (PCMJ), Gard, Le Vigon, 1-8.10.1986, PP; $2 \sigma^{7} \sigma^{7}$ (PCMJ), Vuacl., Roussillon, 16.05, 1989, PP; 1 (PCRS, 17246), Auvergne, Puy d'Eraigne, 30.06. 1974, JFM. - ITALY: $10^{\top}$ (SMFM), Sardinia, 1986, coll.?; 1 O $^{7}$ (PCJM, 18076), Corsica, Venaco, Col de Belle Granajl, 730 m a.s.l., maquis, 26.05.1989, JFM; 1 (PCJM, 17941), Corsica, Étang d'Urbino, coastal scrub, 22.05.1989, JFM. - LIBYA: 1 9 (SMFM, 4150), Tripoli, Vytina, no date and collector name. - GREECE: $10^{7}$ (SMFM), Kelempek Mts, above Neda, 400-500 m a.s.1., Quercus pubescens, 4-7.05.1968, O.V. Helversen \& AS; $10^{7}$ (SMFM), SE part, Kalamafka, 14.05.2009, KE. - CROATIA: 5 우 (MHNG), Istria, Ump. Pula, 9-14.07.1982, S. Heimer. - SLOVENIA: 1 O (SMFM), S of Komen $\left(45^{\circ} 48^{\prime} 20.61^{\prime \prime} \mathrm{N}, 13^{\circ} 45^{\prime} 52.56^{\prime \prime} \mathrm{E}\right)$, Karst, seminatural meadow with stonewalls, bushes and trees, 27.06-5.07.2001, PJ. - TURKEY: 4 OO (MMUM), Konya, Kizilören, 9.07.1992, CF; 1 + (LNMC), Konya, Akşehir, above Dağ Hotel, mixed scrub, herbs and grasses on north-facing limestone slope, 19.09.1993, SJ \& CF; 1 은 (LNMC), Içel, Camlıyayla, Dağ Hotel area $\left(37^{\circ} 10^{\prime} 10^{\prime \prime} \mathrm{N}\right.$, $\left.34^{\circ} 36^{\prime} 02^{\prime \prime} \mathrm{E}\right), 1454 \mathrm{~m}$ a.s.l., limestone cliff, 1.07.1995, SJ \& CF; 1 $\sigma^{7}$ (LNMC), Bağkonak, Sultan Dağlari, Konya, under dwarf hillside herbs, 19.09.1993, CF; 1 ㅇ (LNMC), Corum, c. $4 \mathrm{~km} \mathrm{E} \mathrm{of}$ Elicek $\left(40^{\circ} 18^{\prime} 38^{\prime \prime} \mathrm{N}, 34^{\circ} 42^{\prime} 51^{\prime \prime} \mathrm{E}\right), 1192 \mathrm{~m}$ a.s.l., willow-lined river bank below Quercus hillside, 6.07.1995, SJ \& CF; $1 \sigma^{7}, 3$ 오 (NMPC), Cağlayan, c. $24 \mathrm{~km} \mathrm{SE}$ of Erzincan $\left(39^{\circ} 36^{\prime} \mathrm{N}, 39^{\circ} 42^{\prime} \mathrm{E}\right)$, valley above waterfall, 23-24.06.2002, MR; $10^{7}, 1$ o (ZMTU), Izmir Distr., Yamanlar Daği, on wet ground along a mountain brook and in dry meadow, 24-25.05.1973, PL, F. Önder \& E. Pehlivan; 1 (NHMW), pass N of Silifke, 05.1965, G. Pretzmann. - UKRAINE: $10^{7}, 1$ ( (ZMUM), Kherson Region, Chernomorski Reserve, 06.1985, N.B. Narolski. - ABKHAZIA: 1 \% (ZMUM), nr. Sukhum, left bank of Kelasuri River (c. $3 \mathrm{~km}$ upstream of its mouth), under stones, 11.06.2004, EM. - RUSSIA: $10^{7}$ (SZMN), Chechen Republic, Groznyi, 5-10.07.1988, A.S. Ryabukhin; 1 \% (SZMN), Daghestan, Derbent, 20.05.1989, PD; 1 + (ZISP), Krasnodar Territory, nr. Khosta, 17.07.1975, V.I. Ovtsharenko; $1 \sigma^{7}$ (SZMN), The Crimea, Bakhchisarai, 5-15.06.1993, SV; 1 오 (ZMUM), The Crimea, nr. Simferopol, 26.08.1997, G.B. Reutov; 1 $\sigma^{7}$ (ZMUM), The Crimea, Kanaka, from oak, 25.05.2000, M. On- 
churov; $1 \mathrm{O}^{7}$ (ZMUM), The Crimea, Karadagh, Lobovoi Mt. Range, from pistachio and almond-trees, 29.05.2003, M.M. Kovblyuk. ISRAEL: 2 O$^{7} \sigma^{7}, 1$ \& (HUJI, 15476, 15477), Migwe Yisráel, on citrus, 15.02-20.03.1938, A. Shulov. - SYRIA: $1 \sigma^{7}$ (NMPC), NW Syria, Mashtal Helu nr. Homs, 30.03-3.04.2001, MR; $20^{7} O^{7}$ (NMPC), NW Syria, Slunfeh nr. Latakia, oak forest, 9.04.2001, MR. - AZERBAIJAN: 1 (MMUM), Gabala Distr., Amirvan, 4.06.2001, EG; 1 + (SZMN), Aghstafa Distr., near Poylu, 6.05.1986, PD; 1 क (SZMN), Khachmaz Distr., Mukhtadir, 30.06.1977, PD; 1

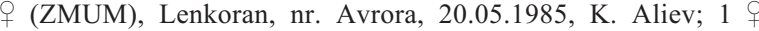
(ZMUM), nr. Kuba, 15.07.1989, PD

COMMENTS. A Mediterranean species, known from France [present data], throughout the Balkans, south Ukraine and Turkey [present data], to the Near East and Azerbaijan [Prószyński, 2003: sub. M. nidicolens; Logunov, Guseinov, 2002: sub. M. nidicolens; present data]. Although the aforementioned records are formally new for France, Libya, Turkey, Israel, Syria and Azerbaijan, this species should have been recorded from these countries under the name $M$. nidicolens (see below).

Macaroeris nidicolens (Walckenaer, 1802)

MATERIAL. SPAIN: 3 O $^{7}$ Or $^{7}$ (SMFM, 2599), Prov. Tarragona, Flix, 05.1915, F. Haas; $10^{7}$ (PCJK), S of Apostoli, 12.05.2003, HDK. - ITALY: 19 (MNHN, 764, identified by E. Simon), 'Ital (Can) (sub Nasdoi)'; 2 우 (MMUM), Lombardia, Bergamo, Dalmine, in wasp's nest, 20.VI.2000, Bugada; $1 O^{7}$ (MMUM), Lombardia, Bergamo, Valgoglio, source in wood, $800 \mathrm{~m}$ a.s.l., 2.VI.1994 Parella, M. Valle. - BOSNIA AND HERCEGOVINA: $20^{7} 0^{7}$ (PCMJ), Neum, 4.05.1988, PP; 1 (PCMJ), Ston, 7.05.1988, PP; $1 O^{7}$ (PCJM, 5416), Hercegovina, Dubrovnik, Petka, Od Gorice, 16.04.1976, JFM; $1 \mathrm{O}^{7}$ (PCRS), headland $\mathrm{N}$ of Lucia, beach $\mathrm{nr}$ Petrovac, 21.05.1984, coll.? - CZECH REPUBLIC: 2 우 (MMUM), southern Moravia, Bzenec, Váté Písky (sand dunes) National Monument, 4.06.1996, V. Růžička. - HUNGARY: 1 O' (SMFM, 2039), 'Sienortorniza' [apparently, Simontornya; label is illegible], 1930 (Roewer's coll.); 1 O', 1 + (MMUM), Szombathely, beating off Pinus nigra twigs, 28.06.1994, C. Szinetár; 5 of (MMUM), Budapest, Sas-hegy, beating off Pinus nigra twigs, 25.05-25.06.1995, C. Szinetár. - GREECE: 1 ㅇ (PCJW), Crete, 'Malrikyks', no date and collector name; $10^{7}$ (SMFM, 33426), Samos island, 1984, Malicky; 1 O$^{T}$ (SMFM, 2041; palpless male), Crete, Mt. Governeto, 1.05.1926, CR; 1 ( (SMFM, 2040), Athens, Akropolis, 05.1926, CR; 1 O (PCJM, 10905), Corfu, Korission, reed beds and sand dunes, 1-12.04.1983, JFM; $1 \mathrm{O}^{7}$ (PCJM, 6730), Halkidiki, Gerakina, 6-8.04.1978, JFM; $3 \sigma^{7} \sigma^{7}$ (PCJM, 6730, 6749, 6831), Halkidiki, Gerakina, 6-14.04.1978, JFM; 5 ○ Oొ $^{7}, 2$ 우 (PCJM, 14818, 16218), Kefallinia (=Kefalonia), Sami, stones and shrubs on roadside, 24-31.05.1987, JFM; 1 O$^{7}$ (PCJM, 14906), Kefallinia (=Kefalonia), Svoronataa, damp area, 30.05.1987, JFM 1 (PCJM, 16154), Kefallinia (=Kefalonia), Sisia, 30.05.1987, JFM; 1 ○ (PCJM, 14876), Kefallinia (=Kefalonia), Assos, Spiridon, stony, grassy area, 27.05.1987, JFM; 1 O $^{7}$ (PCJM, 14711), Kefallinia (=Kefalonia), Pastra, shrubs on trackside, 21.05.1987, JFM; $2 \bigcirc^{7} O^{7}, 1$ ( $($ PCJM, 16387), Kefallinia (=Kefalonia), Atsoupades, gorge, 24-22.05.1987, JFM; 1 O $^{7}$ (PCJM, 14757), Kefallinia (=Kefalonia), Atsoupades, stones and shrubs by gully, 22.05.1987, JFM. - CYPRUS: 3 O$^{7} O^{7}$ (PCJM, 12627), Pano Panayia, 2.05.1982, JFM; $1 \sigma^{7}$ (SMFM), S Crete, Arvi, 12.05.2007, HE \& UK. TURKEY: $2 \sigma^{7} \sigma^{7}, 195$ juv. (LNMC), $20^{7} \sigma^{7}, 1$ (MMUM), c. 10 $\mathrm{km}$ E of Çamlıyayla, grazed ruderal alpine pasture, 11.06.1993, SJ \& CF; 1 (MMUM), Iccel, Tarsus-Camliyayla, c. $3 \mathrm{~km} \mathrm{~S}$ of Sarıkavak, herb-rich limestone hillside, 11.06.1993, SJ \& CF; 1 क (ZMUM), Antalya, Kemer, 50-100 m a.s.l., pine forest edge, 2829.04.2001, K.G. Mikhailov \& E.A. Mikhailova; $1 \sigma^{7}$ (SMFM), Büyükada (the rest of label is illegible), 07.1966, coll.?; 1 क (NHMW), c. $25 \mathrm{~km}$ S of Izmir, road Selçuk-Izmir, 28.05.1969, AR; $5 \mathrm{O}^{7} \mathrm{O}^{\top}$ (NHMW), Namrun, pine forest, 12.05-5.06.1964, FR; 1 + (NHMW), Alihoca, 11.06.1965, FR.
COMMENTS. A European - Mediterranean subboreal species, known from the Iberian Peninsula [Helsdingen, 2014] to Cyprus and Turkey [Helsdingen, 2013; present data]. The records of this species from Azerbaijan [Logunov, Guseinov, 2002] were mistaken and should actually be referred to M. flavicomis (see above). New record for Cyprus.

\section{Marpissa muscosa (Clerck, 1758)}

MATERIAL. SPAIN: $10^{7}$ (SMFM), Madrid, no date and collector name. - GREECE: $10^{7}$ (SMFM, 4160), Crete, Knosos, 06.1926, CR; 1 O $^{7}$ (SMFM), Kelempek Mts, summit region, c. 900 m a.s.1., Quercus pubescens forest, 8.05.1968, JMS.

COMMENTS. A European temperate species, known from France to the Urals [Logunov, Marusik, 2000b].

Marpissa nivoyi (Lucas, 1846)

MATERIAL. MOROCCO: $10^{7}$ (MHNG), Cap Negro, 1-3.08. 1973, Y.G. Billaud. - ALGERIA: $10^{77}$ (PCRB), Wilaya Tipasa, Douaouda, Wadi Mazafran, c. $50 \mathrm{~m}$ a.s.1., pitfall traps in Populus alba forest, 16.12.1986-26.02.1988, RB; 1 O (PCRB), Wilaya Boumerdes, Thenia E., c. $150 \mathrm{~m}$ a.s.l., in litter of Eucalyptus plantation, 18.03.1988, RB; $1 \sigma^{7}$ (PCRB), Wilaya Blida, Atlas Blidéen, Meftha, Djebel Zerouela, c. $480 \mathrm{~m}$ a.s.l., pitfall traps in Quercus suber forest, 11.12.1986-23.06.1987, RB. - FRANCE: $1 \sigma^{\top}$ (SMFM), Dep. Alpes-Maritimes, Col de Turini, c. $50 \mathrm{~km} \mathrm{NNE}$ of Nice, 1500-1650 m a.s.1., 18-19.09.1982, JMS. — GREECE: 1 $\sigma^{7}$ (SMFM), NW coast of Peloponnes, thermal spring of Loutra Killini, 8.06.1998, LM. - RUSSIA: 1 ㅇ (ZMUM), Astrakhan' Region, Lishansky Distr., E of Basinskaya station $\left(46^{\circ} 07^{\prime} 55^{\prime \prime} \mathrm{N}\right.$, $\left.47^{\circ} 13^{\prime} 05^{\prime \prime} \mathrm{E}\right), 18.05 .2012, \mathrm{AG}$.

COMMENTS. A European - Central Asian temperate species [Helsdingen, 2014], known from Morocco and Algeria [present data], eastward to S Kazakhstan and Kyrgyzstan [Logunov, Rakov, 1998]. New records for Algeria and Morocco.

Marpissa pomatia (Walckenaer, 1802)

MATERIAL. TURKEY: $10^{7}, 3$ OP (OUMNH), Istanbul, 150 m a.s.1., beaten off Rhododendron, 20.07.1956, GL. - GEORGIA: $1 \sigma^{7}$ (ZISP, A.No. 301-470), Kolkhida, N. Chkhugunetch, 500$650 \mathrm{~m}$ a.s.1., 3-10.06.1969, EP.

COMMENTS. A trans-Eurasian temperate species, known from France to the Russian Far East and South Korea [Logunov, Marusik, 2000b]. New record for Turkey.

\section{8)}

Mendoza canestrinii (Ninni in Canestrini et Pavesi,

MATERIAL. ALGERIA: $1 \sigma^{7}$ (PCRB), Wilaya Boumerdes, Reghia, $45 \mathrm{~m}$ a.s.1., Olea europea orchard, 3.05.1988, RB. SPAIN: $2 O^{7} O^{7}, 1$ ㅇ (MVHN), Valencia, Confrentes, Cerro Agrás, 6.03.2005, M.A. Ibañez-Orrico. - TURKEY: $10^{\top}$ (LMNM), Adana, c. $5.5 \mathrm{~km} \mathrm{E}$ of Yenice $\left(36^{\circ} 58^{\prime} 01^{\prime \prime} \mathrm{N}, 35^{\circ} 07^{\prime} 17^{\prime \prime} \mathrm{E}\right)$, ruderal plants and grasses on road verge, 4.05.1994, SJ \& CF.

COMMENTS. A trans-Eurasian subboreal-subtropical species, known from Spain to Japan (Hokkaido) [Logunov, Marusik, 2000b]. New record for Algeria.

\section{Menemerus animatus Pickard-Cambridge, 1876}

MATERIAL. ALGERIA: $20^{7} \sigma^{7}, 1$ + (PCRB), Wilaya Adrar, Timimoun, palm grove and garden, 1.04.1989, RB. - EGYPT: 1 古 (SMFM), Cairo, no date and collector name; $2 \sigma^{7} \sigma^{7}, 2$ 오 (NHMW), Ras El-Barr (c. $\left.31^{\circ} 31^{\prime} \mathrm{N}, 31^{\circ} 50^{\prime} \mathrm{E}\right), 1.08 .1982$, HEH. 
COMMENTS. A north-African species (from Algeria to Sudan), known also from the eastern Mediterranean (from Greece to Egypt) [Metzner, 1999; Wesołowska, 1999].

Menemerus davidi Prószyński et Wesołowska, 1999

MATERIAL. JORDAN: $10^{7}$ (SMFM), Wadi Azab, N Amman, 04.2002, J. Hoenung.

COMMENTS. A north-African species, known from Libya, Algeria, Egypt, Israel and Jordan [Wesołowska, 1999; Prószyński, 2003; present data]. New record for Jordan

Menemerus errabundus Logunov, 2010

MATERIAL. ISRAEL: $20^{7} \sigma^{7}$ (NMPC), Sede Boqer, Negev desert, between Beer Sheva and Mitzpe Ramon, 6-29.05.2003, MR.

COMMENTS. Recorded from Iran (Khûzestân: Shush) and Israel only [Logunov, 2010; present data]; it is the first record outside the type locality. New record for Israel.

Menemerus fagei Berland et Millot, 1941

MATERIAL. EGYPT: 1 (NHMW), Asyut (=Assiut; c. $27^{\circ}$ $11^{\prime} \mathrm{N}, 31^{\circ} 10^{\prime} \mathrm{E}$ ), 23.12.1981, HEH; 2 오 (NHMW), El Mansoura (c. $\left.31^{\circ} 04^{\prime} \mathrm{N}, 31^{\circ} 41^{\prime} \mathrm{E}\right), 8.01-19.11 .1981, \mathrm{HEH} ; 1$ 우 (NHMW) Cairo (c. $30^{\circ} 3^{\prime} \mathrm{N}, 31^{\circ} 14^{\prime} \mathrm{E}$ ), no date, HEH; 1 ( NHMW), Ras El-

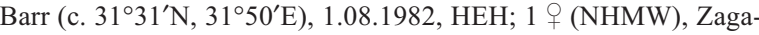
zig $\left(\right.$ c. $\left.30^{\circ} 34^{\prime} \mathrm{N}, 31^{\circ} 30^{\prime} \mathrm{E}\right), 11.11 .1981, \mathrm{HEH}$

COMMENTS. A north-African species, reaching the Near East [Wesołowska, 1999; Prószyński, 2003].

Menemerus semilimbatus (Hahn, 1827)

MATERIAL. MOROCCO: 1 (SMFM), 'Fez. Wez. z... (label illegible)', 27.05.1930, coll.?; $1 \mathrm{O}^{7}$ (SMFM), Taza, c. $5 \mathrm{~km} \mathrm{~S}$ of Sebt-des-Beni-Frassèn, c. $30 \mathrm{~km} \mathrm{NW}$ of Taza $\left(34.20^{\circ} \mathrm{N}, 4.22^{\circ} \mathrm{W}\right)$ $300 \mathrm{~m}$ a.s.l., fallow land with damming wetness, clay, under stones, 22.02.2004, D.W. Wrase. - ALGERIA: $1 \sigma^{7}$ (PCRB), Wilaya Alfer, Ben Aknoun, c. $100 \mathrm{~m}$ a.s.1., terrain vague, 18.05.1988, RB $2 \mathrm{O}^{7} \mathrm{O}^{7}$ (PCRB), Wilaya Iherir, Effeni, c. $100 \mathrm{~m}$ a.s.l., Typha march, 7.02.1986, RB; 1 O$^{7}$ (PCRB), Wilaya El Tarf, El Kala, W bank of Lac Tonga, c. $10 \mathrm{~m}$ a.s.l., wet meadows along lake, 27.03.1988, RB; 1 O $^{7}$ (PCRB), Wilaya Illizi, c. $60 \mathrm{~km} \mathrm{~S}$ of Illizi, Fadnoun, Wad Djerat, among stones in litter of Acacia bark, 10.02.1982, RB; 19 (PCRB), Wilaya Tiz Ouzou, Taguemount Azouz, c. 800 m a.s.1. pitfall traps in Quercus ilex forest, 1.02.1989, RB; 1 O $^{7}$ (PCRB), Wilaya Bejaia, Wadi Daas, c. $15 \mathrm{~m}$ a.s.l., beach nr. the mouth, 22.05.1988, RB; 1 우 (PCRB), Wilaya Alger, Beaulieu, c. $50 \mathrm{~m}$ a.s.l., in garden, no date, RB; $1 \mathrm{O}^{7}$ (PCRB), Wilaya Aïn Defla, between Bordj Emir Khaled and Tarik Ibn Ziad, c. $700 \mathrm{~m}$ a.s.l. border of Wadi Massine, under stones and in litter, 18.05.1988, RB. - PORTUGAL: 1 (FMNH), Mértola (c. $37^{\circ} 38^{\prime} \mathrm{N},-7^{\circ} 40^{\prime} \mathrm{W}$ ), 15.04.2003, PC. - SPAIN: $1 \mathrm{O}^{7}$ (MHNG), Alicante, Castilo de Santa Barbara, 24.03.1978, PH; 1 O, 2 ㅇ (SMFM), La Rabida 05.1959, J. Büddenbrock; 1 † (SMFM), Aljeciras, 05.1959, Büddenbrock; 1 (NMPC), Valencia, Sagunt (=Sagunto) (c. $39^{\circ} 41^{\prime} \mathrm{N}$ $-0^{\circ} 16^{\prime} \mathrm{W}$ ), 26.04.2004, LD; 6 O $^{7} 0^{7}$ (NMPC), Denia, 29.04.2004, LD; $10^{7}, 2$ 우 (NMPC), Calp, 29.04.2004, LD; $10^{7}, 3$ 우 (NMPC), Xávia (=Jávea), NP del Mongú, 29.04.2004, LD; 2 O $^{7} 0^{7}$ (NMPC), Guardamar del Segura, 28.04.2004, LD; 1 O$^{T}$ (NMPC), Besalú, 2.05.2004, LD; $2 \mathrm{O}^{7} \mathrm{O}^{7}$ (NMPC), Valensia, Guadalest (c. $38^{\circ} 41^{\prime} \mathrm{N}$ $0^{\circ} 11^{\prime} \mathrm{E}$ ), 27.04.2004, LD; $1 \mathrm{O}^{7}$ (NMPC), Catalonia, Mataró (c. $\left.41^{\circ} 32^{\prime} \mathrm{N}, 2^{\circ} 27^{\prime} \mathrm{E}\right), 1.05 .2004$, LD. - ITALY: $10^{7}, 1$ (SMFM, 9388), Sardinia, 1936, coll.?; 1 9 (SMFM), Pantelleria, no date and collector name. - LIBYA: 1 + (SMFM, 4150), Tripoli, Vytina, no date and collector name. - GREECE: $10^{7}$ (SMFM), Island Kos, Kastell, 28.04.1968, JMS; $10^{7}$ (LMNM), Lesbos, Parakila (39 102 $\left.19^{\prime \prime} \mathrm{N}, 26^{\circ} 08^{\prime} 35^{\prime \prime} \mathrm{E}\right), 12.07 .1997$, M. Hull; $2 \mathrm{O}^{7} \mathrm{O}^{\top}$ (SMFM, 5152),
Saloniki, no date and collector name; $1 O^{2}, 1+$ (SMFM), Peloponnes, Nauplia, pine forest by the sea, 17.09.1964, M. Eitz; $1 O^{7}, 1$ o (SMFM), S Crete, Keratokampos, 7.05.2008, HE \& UK; $10^{7}$ (SMFM), N Crete, Gouves, 2.05.2008, HE \& UK; 1 ㅇ (SMFM), N Crete, Malia, 11.05.2008, HE \& UK; $1 \sigma^{7}$ (MHNG), Crete, Lassithi, E of Sitia, hill nr. the sea, c. $30 \mathrm{~m}$ a.s.l., 6.05.1985, BH; $10^{7}$, 1 (SMFM), C-Crete, Magarikari, 7.05.2009, KE \& HE; $1 \overbrace{}^{7}, 1$ 9 (SMFM), S coast of Peloponnes, Ligia, beach, dunes and adjacent cultural landscape, 1.06.1998, LM; $1 \sigma^{7}$ (SMFM), S coast of Peloponnes, Foinikous, lime and sand cliffs at seashore, 4.06.1998, LM. - TURKEY: $1 O^{7}$ (PCRS), Akyaka, on rocks behind Cinan Beach, 30.05.1996, ARS; $10^{7}$ (PCRS), Akyaka, Thomsan Villas, on walls of house, 1.06.1996, ARS; 1 + (SMFM), Büyükada (nr. Istanbul, label is illegible), 07-08.1965, coll.?; 1 + (PCSD), among ruins of Ephesus, 25.05.1997, RS; 1 을 (OUMNH), Cavnos, nr. Dalyan, 12.08.2008, R. Gabriel \& L. Hedicker. - AZERBAIJAN: 1 ( $($ SZMN), Astara Distr., between Motlayatat and Bilyasar, Talysh Mts $\left(38^{\circ} 36^{\prime} \mathrm{N}, 48^{\circ} 40^{\prime} \mathrm{E}\right), 600-700 \mathrm{~m}$ a.s.1., Carpinus-Acer-Ouercus forest, 20-22.06.2003, AC; 1 + (SZMN), Ismailly Distr., Ismailly reserve, nr. Buinus Vil. 700 m a.s.1., 25-26.06.2003, AC.

COMMENTS. A Mediterranean species, known from the Canary islands and Morocco to Azerbaijan and N Iran [Rakov, Logunov, 1997; Wesołowska, 1999; Logunov et al., 2002; present data].

Menemerus taeniatus (L. Koch, 1867)

MATERIAL. ALGERIA: 1 (PCRB), Wilaya Skikda, E. Collo, Tamanart, 0-30 m a.s.l., in litter of Quercus suber forest, 20.06.1985, RB. - SPAIN: 1 O' $^{7}$ (NMPC), Salou, 26.04.2004, LD. - TUNISIA: 2 우 (PCJK), North of Beja, under bark of Eucaliptus tree, 8.03.2005, J. Van Keer. - GREECE: $10^{7}$ (SMFM, 5152), Saloniki, no date and collector name. - MACEDONIA: $10^{7}, 1$, (OUMNH), River Vargar, Geogelya wood hut, 11.07.1956, GL. ABKHAZIA: 2 우 (ZMUM), nr. Sukhum, the left bank of Kelasuri River (2-3 km upsteam of the mouth), under stones, 6.06.2004, EM. - AZERBAIJAN: 1 ( (SZMN), Khyzy Distr., c. $12 \mathrm{~km} \mathrm{~W}$ of Kilyazi $\left(40^{\circ} 52^{\prime} \mathrm{N}, 49^{\circ} 12^{\prime} \mathrm{E}\right), 7.06 .2003$, H. Aliev.

COMMENTS. A Mediterranean species, known from Algeria and Portugal to Azerbaijan [Rakov, Logunov, 1997; Wesołowska, 1999; Helsdingen, 2014].

Mogrus logunovi Prószyński, 2000

MATERIAL. EGYPT: 10 OP (NHMW), Ras El-Barr (c. 31 $\left.31^{\prime} \mathrm{N}, 31^{\circ} 50^{\prime} \mathrm{E}\right), 22.04 .1982$, HEH.

COMMENTS. Recorded from Egypt, the Near East, the UAE and Yemen [Prószyński, 2003; Wesołowska, van Harten, 2007, 2010; present data].

Mogrus mirabilis Wesołowska et van Harten, 1994 MATERIAL. EGYPT: 1 \% (NHMW), Sinai (no exact locality), 08.1982, HEH - JORDAN: $10^{7}$ (SMFM), Wadi Rum (=Wadi Ramm; $29^{\circ} 41^{\prime} \mathrm{N}, 35^{\circ} 27^{\prime} \mathrm{E}$ ), Felsnische, HF, 9.04.2004, JA. - IRAQ: $1 O^{7}$ (SMFM), Baiji, flower meadow, 2.04.1986, M. Larl.

COMMENTS. Recorded from Sudan, Yemen, Saudi Arabia, north-eastward to Egypt, Jordan and Iraq [Wesołowska, van Harten, 1994; Logunov, 2004b; present data]. New records for Egypt, Jordan and Iraq.

Mogrus neglectus (Simon, 1868)

MATERIAL. CYPRUS: $20^{77} \mathrm{O}^{7}$ (PCMA), Akrotiri, 27.04.2007, MA; 1 ㅇ (PCDM), Profitis Ulas, 2003, DMC; 2 오 (PCRS), Akrotiri Peninsula, salt marsh, in vegetation, 30.04.2013, coll.? TURKEY: 2 O+ (SMFM), pass c. $9 \mathrm{~km}$ SE of Madanya, no date, coll? - ARMENIA: $10^{7}$ (ZISP, A.No 317-09), Yerevan Area, 'station Negram' (unknown locality), 19.05.1909, N. Bryansky. AZERBAIJAN: $10^{7}, 4$ 우 (ZISP, A.No. 186-1935), c. $50 \mathrm{~km} \mathrm{~S}$ of Yevlakh, nr. Martushevany, 1935, P.A. Veltishev.

COMMENTS. An eastern Mediterranean - Central Asian subboreal-subtropical species, known from 
Greece and Macedonia [Metzner, 1999; Helsdingen, 2014], through Asia Minor [Karol, 1967], to Iran and south Kazakhstan [Logunov et al., 2002]. New record for Cyprus.

Myrmarachne formicaria (De Geer, 1778)

MATERIAL. GREECE: 1 + (PCRG), Makiamos pastures, 27.09.2001, coll.?; 2 O$^{7} \sigma^{7}, 3$ 우 (NHRS), Chania Pref., Gerani, Ksekilomenes River, close to sea, under red debris on ground, 4.05.2000, TK. — TURKEY: 2 O $^{7} \sigma^{7}$ (PCRS), "Mavi kus", Yalikavak, nr. Bodrum, in garden, 27.05.1997, ARS; 1 ( (OUMNH), Van (?), Hahori Bashale, $50 \mathrm{k}$ stream into Zap, among seeds, 30.08.1956, GL; 1 + (NHMW), Yeşilyurt, 2.06.1965, FR; $1 \mathrm{O}^{7}$ (NHMW), Köycegiz, 14.04.1964, FR. - RUSSIA: 1 + (ZISP), Krasnodar Territory, nr. Adler, 29.07.1976, V.I. Ovtsharenko. - ABKHAZIA: 19 (ZMUM), nr. Sukhum, left bank of Kelasuri River (3 km upstream of its mouth), under stones, 11.06.2004, EM; 1 ( (ZMUM), nr. N. Yashtukha, 29.03.1985, A. Markosyan. - GEORGIA: 1 O (SZMN), Kolchida Reserve, alder forest, in litter, 14.04.1988, DL. - AZERBAIJAN: 1 q (ZISP), Lenkoran Area, nr. Alexeevka, rush thicket, 7.07.1983, DL; 1 o (ZISP), same area, nr. Dashdatuk, Bashary river valley, 28.06.1983, DL; $10^{7}$ (ZISP), same area, nr. AzFilial, rush thicket, 2.07.1983, DL; 1 (ZMUM), same area, nr. Gaftoni, 9.05.1985, PD.

COMMENTS. An amphi-Eurasian subboreal species, known from Portugal, eastward to the Caucasus, and then from Cisamuria to Japan [Logunov, Marusik, 2000b].

Neaetha absheronica Logunov et Guseinov, 2002

MATERIAL. GREECE: $1 \sigma^{7}$ (PCJK), Macedonia, Strymon FPlain, c. 55 m a.s.1., 21.05.2009, J. Van Keer; 1 (PCJW), Kaimeni, Plaea, Santoria, 28.05.<no year $>$, J. Wunderlich. - ABKHAZIA: $1 \sigma^{7}$ (ZMUM), nr. Sukhum, left bank of Kelasuri River (2-3 $\mathrm{km}$ upstream of its mouth), 5.06.2004, EM. - AZERBAIJAN: $\sigma^{7}$ (MMUM), Lenkoran Area, Liman (c. $\left.38^{\circ} 50^{\prime} \mathrm{N}, 48^{\circ} 49^{\prime} \mathrm{E}\right), 22.05$. 2003, EG.

COMMENTS. An eastern Mediterranean species, known from Greece and Macedonia [Fiser, Azarkina, 2005; present data], through Turkey [Coşar et al., 2014], to Azerbaijan [Logunov, Guseinov, 2002; Marusik et al., 2004]. New records for the NW Caucasus and formally for Greece; yet, some of the earlier records of Neaetha membrosa from Greece [see Bosmans, Chatza$\mathrm{ki}, 2005$ ] may actually belong to $N$. absheronica.

The $q$ illustrated by Metzner [1999] seems to also belong to $N$. absheronica, as it is identical to the 9 from Greece (Santoria) listed above.

Neaetha membrosa (Simon, 1868)

MATERIAL. SPAIN: $1 \sigma^{7}$ (NMPC), Denia, 29.04.2004, LD. - ITALY: $1 \mathrm{O}^{\top}$ (SMFM), Sicily, c. $2 \mathrm{~km} \mathrm{~W}$ of Castelbuono, stony SE-slope, moist grass beneath grainfileds with Fraxinus ornus (under stones), 31.03.1957, HK.

COMMENTS. It is a west-Mediterranean species, known from the Iberian Peninsula to Germany and Greece [Helsdingen, 2014].

Neon convolutus Denis, 1937

MATERIAL. ALGERIA: $3 \bigcirc^{7} \sigma^{7}, 1$ ( 1 (PCRB), Wilaya Oran, forest nr. Msila, $400 \mathrm{~m}$ a.s.1., sieving Quercus suber litter and among stones and Oxalis, 25.04.1984, RB. - SPAIN: $10^{7}$ (NMPC), Valensia, Guadalest (c. $38^{\circ} 41^{\prime} \mathrm{N}, 0^{\circ} 11^{\prime} \mathrm{E}$ ), 27.04.2004, LD.

COMMENTS. A west-Mediterranean species, restricted to Algeria, the Iberian Peninsula and France [Logunov, 2004a; Helsdingen, 2014; present data]. The male of $N$. convolutus from Spain is identical to the holotype from Algeria illustrated by Logunov [2004a: figs 7-8]. As was already assumed by the latter author, the name of $N$. convolutus could be synonymous with N. rayi (Simon, 1875). New record for Spain.

Neon levis (Simon, 1871)

MATERIAL. MONTENEGRO: $1 \sigma^{\top}, 1$ (SMFM), Rumija Mts, Castanea sativa forest nr. Ostros $\left(42^{\circ} 5^{\prime} 20.9^{\prime \prime} \mathrm{N}, 19^{\circ} 17^{\prime} 25.4^{\prime \prime} \mathrm{E}\right)$, sieving, 5.05.2006, AS. - TURKEY: $10^{7}, 1$ + (NHMW), Tekketal, SW Akşehir, 1100-1200 m a.s.1., pine forest with Quercus coccifera, Corylus, etc., 11.06.1967, JG, FR \& AR; $10^{7}$ (NHMW), Namrun, pine forest, 12.05-5.06.1964, FR. - SYRIA: $2 O^{7} O^{7}$ (NMPC), NW Syria, castle Qalaat al Kahf nr. Qadmus, 5-7.04.2001, MR. - GEORGIA: 1 (ZMUM), c. $14 \mathrm{~km}$ E of Tkibuli, Mukhura, Fagus \& Quercus forest, in litter, 23.05.1981, SG.

COMMENTS. A Euro-Siberian - Cenral Asian subboreal species, known from Portugal to Transbaikalia, southward to Tajikistan and Iran [Logunov, Rakov, 1998; Logunov et al., 2002]. New record for Syria.

Neon rayi (Simon, 1875)

MATERIAL. PORTUGAL: $1 \sigma^{\top}$ (FMNH), Vale Garcia (c. $\left.39^{\circ} 33^{\prime} \mathrm{N}, 8^{\circ} 35^{\prime} \mathrm{W}\right), 21.05 .2002$, PC. - TURKEY: 1 (ZMUM), Aksu, valley next to Zindan cave, 6.07.2003, MR.

COMMENTS. A Euro-Siberian subboreal species, known from Portugal [Helsdingen, 2014] to the mountains of S Siberia [Logunov, Marusik, 2000b], but records of this species are rare elsewhere. New record for Turkey.

The studied female from Turkey is identical to that illustrated by Metzner [1999: table 31], but differs in the conformation of its copulatory organs (viz., the arrangement of spermathecal loops) from the females of $N$. rayi illustrated by other authors [cf. Nentwig et al., 2014]. The conspecifity and taxonomic status of $N$. rayi from Greece and Turkey require a special attention in the future.

\section{Neon reticulatus (Blackwall, 1853)}

MATERIAL. FRANCE: 1 ( SMFM), Dep. Alpes-Maritimes, Col de Turini, c. $50 \mathrm{~km}$ NNE of Nice, $1500-1650 \mathrm{~m}$ a.s.1., 1819.09.1982, JMS; 1 + (SMFM), Corsica, Col de Verde, c. $1060 \mathrm{~m}$ a.s.1., mixed forest, 9.05.1982, JMS. - ITALY: Trentino, Molina di Ledro, 670-680 m a.s.1., 6-21.09.1990, JMS. - SLOVENIA: 3 우 (SMFM), Rakov Skaèjen (?), 18.07.1974, JMS; 1 (SMFM), Slioica, Mt nr. Zirknitz, 800-1000 m a.s.1., 17.07.1974, JMS. ROMANIA: $1 \sigma^{7}$ (NMPC), Bihor-zona Padiš, 15-21.07.2001. MR. - TURKEY: $2 \mathrm{O}^{7} \mathrm{O}^{7}$ (NHMW), c. $5 \mathrm{~km}$ NE of Ulubey, c. $350 \mathrm{~m}$ a.s.1., decidous forest of Castanea, Quercus, Robinia, Carpinus, etc., 2.06.1967, JG, FR \& AR; 1 क (MHNG), Sinop, Lala près de Sinop, 20.05.1976, C. Besuchet \& I. Löbl. - RUSSIA: 1 ㅇ (ZMUM), Chechnya, c. $5 \mathrm{~km} \mathrm{~W}$ of Shaami-Yurt, c. $25 \mathrm{~km} \mathrm{~W}$ of Grozny, Acer forest, in litter, 6.06.1982, SG. - GEORGIA: 1 우 (ZMUM), S of Gudauri, Krestovyi Pass, $1800 \mathrm{~m}$ a.s.1., Rododendron litter, 4.06.1982, SG. - AZERBAIJAN: 2 우 (SZMN), Lenkoran Area, Lenkoran Reserve and Apo (c. $38^{\circ} 38^{\prime} \mathrm{N}, 48^{\circ} 47^{\prime} \mathrm{E}$ ), 23 28.05.2003, E.F. Guseinov; 1 ㅇ (SZMN), same area, Lenkoran Reserve, in forest litter, 3.07.1983, DL; $10^{7}, 1$ (ZMUM), Zakataly Reserve, SE slope of Rochigel Mt., upper forest margin, 2000 $2300 \mathrm{~m}$ a.s.l., 13-14.06.1986, KM; 1 ( (ZMUM), same reserve, nr. Dzhar, along Terik-chai, $1000 \mathrm{~m}$ a.s.l., Fagus forest, in litter, 8.06. 1986, KM.

COMMENTS. A Holarctic temperate species, known from the Iberian Peninsula [Helsdingen, 2014] to the Kuril Islands and Japan [Logunov, Marusik, 2000b]; in Nearctic, from Alaska and Yukon, southward to Oregon [Dondale et al., 1997]. 
Pellenes brevis (Simon, 1868)

MATERIAL. CYPRUS: $10^{7}$ (LMNM), c. $8 \mathrm{~km}$ NE of Stavros tis Psokias, Paphos forest, 8.09.1997, CF; $1 \mathrm{O}^{7}$ (SZMN), N of Paphos, Lara, dry Juniperus meadow, 14.04.1997, V. Rinne.

COMMENTS. A Mediterranean species, known from Spain [Helsdingen, 2014] to the Crimea [Logunov et al., 1999]. New record for Cyprus.

\section{Pellenes geniculatus (Simon, 1868)}

MATERIAL. SPAIN: $1 O^{7}, 2$ 우 (NMPC), Xávia (=Jávea), NP del Mongú, 29.04.2004, LD. — TURKEY: $3 \mathrm{O}^{7} \mathrm{O}^{7}, 2$ 2 +P (PCSD), S of Denizli, Kenzikbelli pass, 29.05.1997, RS; 1 + (PCLJ), Irmasan Gec, 27.05.2004, LJ; 2 O $^{7} \sigma^{7}, 3$ 우우 (NHMW), Akșehir, Sültandağ, 5-26.05.1960, FR. - CYPRUS: $1 \sigma^{7}$ (LMNM), Trodos, Trout farm Agios Nikolads Steyis, River bank, 4.09.1997, CF. - SYRIA: $10^{7}$ (NMPC), NW Syria, castle Qalaat al Hosn nr. Homs, $3-$ 4.04.2001, MR.

COMMENTS. A European - Central Asian subboreal species, known from the Iberian Penninsula [Helsdingen, 2014] to Tajikistan [Logunov et al., 1999]. New record for Cyprus.

Pellenes moreanus Metzner, 1999

MATERIAL. GREECE: 1 (PCRG), Thasos, Ipsarion $\left(40^{\circ} 39^{\prime} \mathrm{N}\right.$, $24^{\circ} 40^{\prime}$ E), c. $820 \mathrm{~m}$ a.s.1., 27.05.2003, AF.

COMMENTS. Continental Greece only [Metzner, 1999; Bosmans, Chatzaki, 2005].

\section{Pellenes ostrinus (Simon, 1868)}

MATERIAL. GREECE: 2 \%o (MMUM), Lesbos, Stavros $\left(39^{\circ} 02^{\prime} 12^{\prime \prime} \mathrm{N}, 26^{\circ} 16^{\prime} 45^{\prime \prime} \mathrm{E}\right)$, c. $158 \mathrm{~m}$ a.s.1., damp, rocky and grazed roadside field with low shrubs among grass, 21.04.1997, SJ; 1 웅 (LMNM), Lesbos, Angliki Skala, nr. Krioneri $\left(39^{\circ} 11^{\prime} 35^{\prime \prime} \mathrm{N}, 26^{\circ}\right.$ $18^{\prime} 33^{\prime \prime} \mathrm{E}$ ), pine woodland over mixed scrub alongside river, 24.04 1997, SJ; $10^{7}$ (MMUM), Lesbos, Vatera (39 $\left.01^{\prime} 13^{\prime \prime} \mathrm{N}, 26^{\circ} 10^{\prime} 53^{\prime \prime} \mathrm{E}\right)$ 20.04.1997, SJ. - TURKEY: 1 † (NMPC), Çağlayan, c. $24 \mathrm{~km} \mathrm{SE}$ of Erzincan $\left(39^{\circ} 362 \mathrm{~N}, 39^{\circ} 422 \mathrm{E}\right)$, valley above waterfall, 2324.06.2002, MR; 1 + (ZMTU), Izmir Distr., Yamanlar Vil., in dry meadow on a slope, 25.05.1973, PL, F. Önder \& E. Pehlivan. AZERBAIJAN: $1 \sigma^{7}$ (MMUM), Lenkoran Area, Mamusta (c. $\left.38^{\circ} 38^{\prime} \mathrm{N}, 48^{\circ} 47^{\prime} \mathrm{E}\right), 27.05 .2003$, N. Snegovaya; $1 \sigma^{7}$ (ZMUM), Lerik, Amburdara, 12.05.1985, PD; 1 O$^{7}$ (ZISP, A.No. 273), Lerik, 12.05.1909, coll.?; 1 (ZISP, A.No. 360-1933), Nakhichevan' Area, dry valley $3 \mathrm{~km}$ of Ordubat, 15.07.1933, D. Znoiko.

COMMENTS. An eastern Mediterranean species, known from Greece (both mainland and islands) to Israel and Azerbaijan [Metzner, 1999; Prószyński, 2003; present data]. The records of Pellenes diagonalis ( $\mathrm{Si}$ mon, 1868) based on the females from Greece [Metzner, 1999], Turkey [Topçu et al., 2006] and Syria [Prószyński, 2003] are likely to actually belong to $P$. ostrinus as well.

Pellenes seriatus (Thorell, 1875)

MATERIAL. RUSSIA: $1 \sigma^{7}$ (ZISP, A.No.179), Daghestan, Kizlyar, 9-10.05.1925, Kirichenko.

COMMENTS. A European - Central Asian subborel species, known from Greece to Iran and southeast Kazakhstan [Logunov et al., 1999; Logunov, Marusik, 2000b].

\section{Philaeus chrysops (Poda, 1776)}

MATERIAL. MOROCCO: 1 (SMFM), Azrou (c. $33^{\circ} 27^{\prime} \mathrm{N}$, $\left.-5^{\circ} 13^{\prime} \mathrm{W}\right), 29.05 .1930$, coll.? - ALGERIA: $20^{7} \sigma^{7}, 1$ ( 9 (PCRB), Wilaya Aïn Defla, between Bordj Emir Khaled and Tarik Ibn Ziad, c. $700 \mathrm{~m}$ a.s.1., border of Wadi Massine, under stones and in litter,
18.05.1988, RB; $2 \mathrm{O}^{7} \mathrm{O}^{7}, 1$ 오 (PCRB), Wilaya Tizi Ouzou, Massiff Djurdjura, Tala Guilef, c. $1800 \mathrm{~m}$ a.s.l., among stones in mixed Cedrus and Quercus ilex forest, 25.11.1985, RB. - SPAIN : $10^{7}$ (MVHN), Valencia, Alicante, Vall d'Ebo, Exterior Avenc Ample, 25.05.2005, SM; 1 क (MHNG), Barcelona, 12.1981, A. Senglet \& PH. - LIBYA: $10^{7}$ (SMFM, 4150), Tripoli, Vytina, no date and collector name. - GREECE: 1 \& (SMFM), Island Kos, Kastell, 28.04.1968, JMS; 1 + (SMFM), Kelempek Mts, above Neda, 400$500 \mathrm{~m}$ a.s.1., Quercus pubescens, 4-7.05.1968, O.V. Helversen \& AS; $1 \mathrm{O}^{7}$ immature (PCRG), Thasos, Ipsarion $\left(40^{\circ} 39^{\prime} \mathrm{N}, 24^{\circ} 40^{\prime} \mathrm{E}\right)$, $820 \mathrm{~m}$ a.s.1., 27.05.2003, AF; $1 \mathrm{O}^{7}, 2$ juv. (SMFM, 4151), Crete,

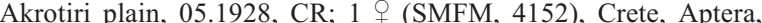

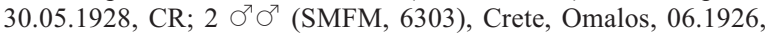
CR; 2 오 (SMFM, 5152), Saloniki, no date and collector name; 2 $\sigma^{7} \sigma^{7}$ (SMFM), Crete, valley NW of Curna lake, rocky, dense bushes, Ficus, Quercus coccifera, on stones, 15.04.1958, HK; $10^{7}$ (SMFM), Crete, c. $8 \mathrm{~km} \mathrm{~W}$ of Chania, E-shore of reservoir nr. Ajia, Cystus and Lavendula, 23.04.1958, HK; $4 \mathrm{O}^{7} \mathrm{O}^{7}$ (SMFM), N Crete, Gouves, 1-4.05.2008, HE \& UK; $1 \mathrm{O}^{7}$ (SMFM), S Crete, Keratokampos, 7.05.2008, HE \& UK; 1 ( (SMFM), S Crete, Arvi, 12.05.2007, HE \& UK; 1 क (SMFM), S Crete, Miliaradon, 11.05.2007, HE \& UK; 1 o (MHNG), Naxos, au pied du mont Zeus, 420-490 m a.s.1., 14.05.1985, CL. - CROATIA: 1 (SMFM), PrimorijeGorski kotar, Rab Isl., hand collecting, 12-15.05.2008, JA \& JM. - TURKEY: 4 O $^{7} 0^{7}$ (PCRS), Akyaka, Sulemin's castle, under stones, 28.05.1996, ARS; $1 \sigma^{7}$ (LMNM), Antalya, c. $31 \mathrm{~km} \mathrm{~N}$ of Alanya, Aksali Dağlari, 19.07.1992, CF; 1 O $^{7}$ (LMNM), Ìsparta, c. 4 km E of Ëgridir, 8.07.1992, SJ; $1 \sigma^{7}$ (LMNM), Kayseri, c. 31 km $\mathrm{S}$ of Duvalli, rocky outcrop with Euphorbia sp and mixed scrub, 15.06.1993, SJ \& CF; 1 + subadult (LMNM), Konya, Sultan Dâglari, c. $7 \mathrm{~km}$ NE of Bağkonak, 8.07.1992, CF; $3 \sigma^{7} \sigma^{7}$ (LMNM), Kayseri, Demirkazık, 12-13.06.1993, CF; 1 O$^{7}, 1$ (LMNM), Narlikuyu, Mersin, garigue-type vegetation on limestone outcrop, under pine needles and scattered short herbs, 9.06.1993, SJ \& CF; 1 O $^{7}$ (LMNM), Içel, Çamliyayla, Dağ Hotel area $\left(37^{\circ} 10^{\prime} 10^{\prime \prime} \mathrm{N}, 34^{\circ}\right.$ $\left.36^{\prime} 02^{\prime \prime} \mathrm{E}\right), 1454 \mathrm{~m}$ a.s.1., limestone cliff, 1.07.1995, SJ \& CF; $1 \mathrm{O}^{7}$ (LMNM), Bağkonak, Sultan Dağlari, Konya, under dwarf hillside

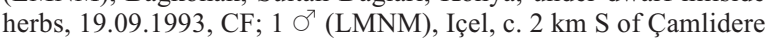
$\left(36^{\circ} 52^{\prime} 12^{\prime \prime} \mathrm{N}, 34^{\circ} 24^{\prime} 37^{\prime \prime} \mathrm{E}\right)$, rocky disuded quarry at side of road, dominated by Euphorbia, Mullein, Cistus and Rubus, 30.06.1995, SJ \& CF; 4 O $^{7} \sigma^{7}, 2$ 우 (LMNM), Içel, Narlıkuyu (36 $26^{\prime} 04^{\prime \prime} \mathrm{N}, 34^{\circ}$ $06^{\prime} 44^{\prime \prime}$ E), garrigue vegetated limestone dominated by Pinus, 5.06 . 1994, MB; 1 ( (LMNM), Kayseri, c. 1 km E of Dörtyal, Çol Gölü $\left(38^{\circ} 27^{\prime} 40^{\prime \prime} \mathrm{N}, 35^{\circ} 10^{\prime} 05^{\prime \prime} \mathrm{E}\right), 11.05 .1994$, SJ \& CF; $2 \mathrm{O}^{\prime} 0^{7}$ (LMNM), Corum, c. $4 \mathrm{~km} \mathrm{E}$ of Elicek $\left(40^{\circ} 18^{\prime} 38^{\prime \prime} \mathrm{N}, 34^{\circ} 42^{\prime} 51^{\prime \prime} \mathrm{E}\right), 1192 \mathrm{~m}$ a.s.1., willow-lined river bank below Quercus hillside, 6.07.1995, SJ \& CF; 1 + (OUMNH), Van, 15k Bosskale Hakari on grass slope, 21.08.1956, GL; 2 우 (OUMNH), Bitlis, Nemsut Dağ, ca 2750 m a.s.l., lake in garden (under stones), 12.08.1956, GL; 3 +o (OUM$\mathrm{NH}$ ), Van, $30 \mathrm{~km}$ of Van Tatvan, at $7500 \mathrm{ft}$ on hillside of Artor Dağ, under stones, 2.09.1956, GL; 1 क (OUMNH), Van, c. $75 \mathrm{~km}$ Van Tatvan, under stones and rocks, Pelli Dağ, $7400 \mathrm{ft} ., 4.09 .1956$, GL; 1 \& (ZMUM), Antalya, Kemer, pine forest, 50-100 m a.s.l., 23-24.04.2001, KM; 1 \% (NMPC), Aksu, Dedegol Mts E of Zindan, pastures, 8.07.2003, MR; 1 + (NMPC), Camlik, nr. Seydisehir, pastures on limestone, 12.07.2003, MR; 1 ( NMPC), Yakaavsar nr. Aksu, Dedegol Mts, Pinus forest below Raham Mt., $2206 \mathrm{~m}$ a.s.1., 9.07.2003, MR; $2 \mathrm{O}^{7} \mathrm{O}^{7}, 1$ ( (NMPC), Nr. Pazar, c. $24 \mathrm{~km} \mathrm{~W}$ of Tokat $\left(40^{\circ} 16^{\prime} \mathrm{N}, 36^{\circ} 17^{\prime} \mathrm{E}\right)$, Ballica, Nr. Mağarasi cave, 29.06-1.07.2002, MR; 1 + (NMPC), Tunceli $\left(39^{\circ} 06^{\prime} \mathrm{N}, 39^{\circ} 33^{\prime} \mathrm{E}\right)$, Munsur river, 22.06.2002, MR; 2 juv. (ZMTU), Erzincan Distr., Gatalarmut, stone bed at small river, 12.09.1971, PL; 1 ( SMFM), Tal zum Coruh Nehri, c. $4 \mathrm{~km}$ WNW of Ispir, on the road IyidereIspir $\left(40^{\circ} 29^{\prime} \mathrm{N}, 40^{\circ} 57^{\prime} \mathrm{E}\right), 1320 \mathrm{~m}$ a.s.1., 26-27.07.1988, coll. Zoologie THD; $1 \mathrm{O}^{7}$ (SMFM), Kaymaz, mouth of the left tributary of Coruh Nehri, c. $18 \mathrm{~km} \mathrm{SW}$ of Yusufeli $\left(40^{\circ} 43^{\prime} \mathrm{N}, 41^{\circ} 24^{\prime} \mathrm{E}\right)$, 28 29.07.1988, coll. Zoologie THD; 1 + (PCLJ), Irmasan Gec, 27.06. 2004, LJ; 2 우 (PCLJ), Yarpuz, 27.05.2004, LJ; 1 ㅇ (NHMW), Hereke, c. $23 \mathrm{~km} \mathrm{~W}$ of Ižmit, $50-100 \mathrm{~m}$ a.s.l., olive plantation (under stones and in grass), 25.05.1967, JG, FR \& AR; 2 우 (NHMW), Yozgat, 1300-1600 m a.s.1., 28-29.05.1967, Y. Eiselt; $1 \mathrm{O}^{7}$ (NHMW), Tekketal, SW Akșehir, 1100-1200 m a.s.l., pine forest with Quercus coccifera, Corylus, etc., 11.06.1967, JG, FR \& 
AR; 1 ( $(\mathrm{NHMW})$, Niğde, c. $125 \mathrm{~m}$ a.s.1., in park, 8.06.1967, JG, FR \& AR; $1 \mathrm{O}^{7}, 1$ ( $\mathrm{NHMW}$ ), c. $20 \mathrm{~km} \mathrm{~N}$ of Turhal, 800-1000 m a.s.1., pine forest, 4.06.1967, JG, FR \& AR; 1 (NHMW), northern slope $\mathrm{S}$ of Amasya, c. $800 \mathrm{~m}$ a.s.l., orchard with dense vegetation, 30.05.1967, JG, FR \& AR; 1 \%, 1 क (NHMW), c. $30 \mathrm{~km} \mathrm{SW}$ of Samsun, Samsun-Merzifon Rd, c. $500 \mathrm{~m}$ a.s.1., deciduous forest of Quercus, Cotinus, Ligustrum, Prunus, Rosa, etc., 3.06.1967, JG, FR \& AR; 1 ( NHMW), c. $20 \mathrm{~km} \mathrm{NW}$ of Ankara, c. $900 \mathrm{~m}$ a.s.1., steppe, 29.05.1967, JG, FR \& AR; 1 + (NHMW), road between Eskischir and Ankara, c. $1050 \mathrm{~m}$ a.s.1., 27.05.1972, K. Bilek \& FR; 1 (NHMW), c. $40 \mathrm{~km}$ on the road to Beyşehir, hill area, 18.05. 1969, AR; 1 of (NHMW), c. $20 \mathrm{~km}$ of the road Anamur-Alanya, along the road to Ermenek, in lush vegetation, 16.05.1969, AR; 1 juv. (NHMW), Pozante, river valley on slopes with Pinus sp. and bushes, 8.05.1969, AR; $1 \mathrm{O}^{7}$ (NHMW), c. $10 \mathrm{~km}$ of the road Mersin-Silifke, 13.05.1969, AR; $4 O^{7} O^{7}$ (NHMW), Akşehir, Sültandağ, 27.04-14.05.1960, FR; 1 ○', 1 + (NHMW), Namrun, pine forest, 12.05-5.06.1964, FR; 1 o', 2 우 (NHMW), Bülgamaden, 12-14.06.1965, FR; 1 + (NHMW), c. $30 \mathrm{~km} \mathrm{~N}$ of Bilecik, 7.05.1965, FR; $1 \sigma^{7}$ (NHMW), Alihoca, 11.06.1965, FR; $1 \sigma^{7}$ (MHNG), nr. Kemer, 22-30.05.1993, PH. — SYRIA: 1 O $^{7}$ (NMPC), NW Syria castle Qalaat al Hosn nr. Homs, 3-4.04.2001, MR. - JORDAN: 1 o (SMFM), Dana Nature Reserve, Wadi Dana $\left(30^{\circ} 412\right.$ N, $35^{\circ} 372$ E), under stones, 16.04.2004, JA \& JM. - GEORGIA: $3 \sigma^{7} \sigma^{7}, 3$ 우 (SZMN), nr. Dzegvi, 17.06.1982, E. Budris; 1 O $^{7}, 1$ q (ZISP, A.No. 253), Tbilisi, 1910, K. Satulin. - RUSSIA: $10^{7}$ (SZMN), the Crimea, Bakhchisarai, 24-26.06.1993, SV; 1 o (ZMUM), Daghestan, Kiziyurt Distr., Kakayurt river valley, 20.05.1981, O.V. Voltzit; 2 우 (ZISP, A.No. 179), Daghestan, Kizlyar, 9-10.05.1925,

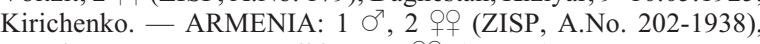
Megri, 28.04.1938, A. Rikhter; 2 오 (SZMN), nr. Sevan town, under stones, 31.07.1983, DL; 1 Ơ $^{\top}$ (ZISP, A.No. 617), Elenovka on Gokcha Lake, 22.07.1922, AD. - AZERBAIJAN: 1 O (ZMUM), Lenkoran Area, Hyrkan Reserve, 21.06.1985, PD; $1 \mathrm{O}^{7}$ (ZISP, A.No. 237-932), same area, nr. Alexeevka, 28.06.1932, D.V. Znoiko; 1 (ZISP, A.No. 360-1933), Nakhichevan' Area, nr. Ordubat, 6.07.1933, D.V. Znoiko; 1 + (ZISP, A.No. 237-1932), Talysh, Zuvand, nr. Kyzyl-Yurda, 9.08.1932, D.V. Znoiko; 1 ○', 1 \& (ZISP), Khachmas Distr., nr. Nabran', 26.07.1986, PD; 1 \& (ZISP, A.No. 34909), nr. 'Elizavetopol' (now Gyandzha), 05.1909, B. Volchansky.

COMMENTS. A trans-Palaearctic subboreal-subtropical species [Logunov, Marusik, 2000b].

Phintella castriesiana (Grube, 1861)

MATERIAL. RUSSIA: $1 O^{7}$ (ZISP), Krasnodar Territory, nr. Maikop, 17.06.1975, V.I. Ovtsharenko; 5 우 (ZISP), same territory, nr. Khomuty, 15.08.1982, E. Prokofieva. - GEORGIA: 1 o (ZMUM), E of Poti, Chaladidi, alder-oak forest, on swamp in litter, 13.04.1983, SG; $1 \sigma^{7}, 1$ ( (ZISP, A.No. 301-470), Kolkhida, N Chkhugunetch, 500-650 m a.s.1., 3-10.06.1969, EP. - TURKEY $1 \mathrm{O}^{\top}$ (LMNM), Içel, c. $3 \mathrm{~km}$ E of Darıpınarı, beating overhanging heather, 12.06.1993, SJ \& CF; $10^{7}$ (NHMW), c. $5 \mathrm{~km} \mathrm{NE}$ of Ulubey, c. $350 \mathrm{~m}$ a.s.1., deciduous forest of Castanea, Quercus, Robinia, Carpinus, etc., 2.06.1967, JG, FR \& AR; 1 O $^{7}$ (NHMW), Namrun, pine forest, 12.05-5.06.1964, FR. - AZERBAIJAN: 19 (SZMN), Lenkoran Area, nr. Osakudzha, under bark of logs, 14.07.1983, DL; $1 \mathrm{O}^{\top}$ (ZISP), same area, nr. Alexeevka, on brambery, 27.06.1983, DL; 2 O O $^{7}$ (ZISP), same area, nr. Dashdatuk, Bashary river valley, 28.06.1983, DL; 1 + (ZMUM), Kakhsky Distr., Vandam, 17.06.1986, PD

COMMENTS. An amphi-Eurasian subboreal species, known from Spain, throughout the Mediterranean, including Asia Minor [e.g., Özşen, Türkeş, 2009; Coşar et al., 2014], to the Caucasus and NW Iran, and then appearing again in Manchuria and Japan [Logunov, Marusik, 2000b].

Phlegra bresnieri (Lucas, 1846)

MATERIAL. MOROCCO: $1+$ (PCRB), Ifrane Prov., Azrou, c. $1250 \mathrm{~m}$ a.s.l., wet meadow along an Wadi $\mathrm{S}$ of the town, among stones, Juncus and Scirpus and sweeping vegetation, 11.05.1984,
RB; $1 \mathrm{O}^{7}$ (PCRB), same prov., Dayet (Lake) Iffrah, c. $1780 \mathrm{~m}$ a.s.1., among stones at the border of the lake with no vegetation due to cattle grazing, 11.05.1984, RB; $1 \mathrm{O}^{7}$ (PCRB), Tetouan Prov., c. 10 $\mathrm{km} \mathrm{E}$ of Chechaouen, c. $500 \mathrm{~m}$ a.s.l., in litter of open Quercus sober forest, 15.05.1984, RB. - ALGERIA: 1 + (PCRB), Wilaya El Tarf, El Kala, $\mathrm{N}$ of Lac Tonga, c. $10 \mathrm{~m}$ a.s.l., pitfall traps in Pinus halepensis forest in dunes, 27.03.1988, RB; 1 o (PCRB), Wilaya Alger, El Harrach, I.N.A., c. 25 m a.s.l., pitfall in rough grassland in park, 31.12.1985, RB; $3 \sigma^{7} \sigma^{7}$ (PCRB), same locality, 4.04.1983, RB; 2 O $^{7}$ O $^{7}$ (PCRB) \& 2 O $^{\top} \sigma^{\top}$ (ZMUM), same locality, 16.06.1985, RB; 1 O (PCRB), Wilaya Blida, Chrea, c. $1550 \mathrm{~m}$ a.s.1., mountain grassaland, 10.06.1985, RB; $1 \sigma^{7}$ (PCRB), same locality, c. $650 \mathrm{~m}$ a.s.l., in litter of Pinus halepensis forest, 18.04. 1985, RB; 1 \& (PCRB), Wilaya Mascara, Col Ain Guergoum along N14, c. $700 \mathrm{~m}$ a.s.1., among stones, 25.04.1984, RB; $1 \mathrm{O}^{7}$ (PCRB), Wilaya Tlemcen, pass Zarifète, c. $1150 \mathrm{~m}$ a.s.l., young ( $\mathrm{m}$ high) Quercus ilex forest, 24.04-6.05.1984, RB; 1 + (PCRB), Wilaya Tlemcen, c. $5 \mathrm{~km} \mathrm{~W}$ of Tlemcen, along N7, c. $850 \mathrm{~m}$ a.s.1., among stones and in grass along very small rivulet, 23.04.1984, RB; $1 \mathrm{O}^{7}$ (PCRB), Wilaya Boumerdes, Zemmouri, c. $10 \mathrm{~m}$ a.s.1., pitfall traps in dunes, 26.10. 1984, RB; 1 + (PCRB), Wilaya Aïn Defla, between Bordj Emir Khaled and Tarik Ibn Ziad, c. 700 m a.s.l., border of Wadi Massine, under stones and in litter, 18.05.1988, RB; $1 \sigma^{7}$ (SZMN), Wilaya Saida, c. $40 \mathrm{~km} \mathrm{SE}$ of Saida, forestry headquater in Oum Djerane, c. $1300 \mathrm{~m}$ a.s.l., pitfall traps in maquis of Quercus forest, 1.10.1984, RB; $2 \mathrm{O}^{7} \mathrm{O}^{7}$ (SZMN), Wilaya Boumerdes, Zemmouri, c. 5 m a.s.l., dunes, 22.03.1985, RB. - PORTUGAL: $4 \bigcirc^{7} O^{7}, 3$ 우 (FMNH), Mértola (c. $37^{\circ} 38^{\prime} \mathrm{N},-7^{\circ} 40^{\prime} \mathrm{W}$ ), on the ground, 3.03-13.08.2003, PC; $1 \mathrm{O}^{7}$ (FMNH), Braciais (c. $37^{\circ} 38^{\prime} \mathrm{N}$, $\left.-7^{\circ} 34^{\prime} \mathrm{W}\right)$, pitfall traps, 9.04.2003, PC; $6 \mathrm{O}^{7} \mathrm{O}^{7}(\mathrm{FMNH})$, Corredoura $\left(\right.$ c. $\left.37^{\circ} 45^{\prime} \mathrm{N},-7^{\circ} 39^{\prime} \mathrm{W}\right)$, pitfall traps, 4.06-2.07.2003, PC; 2 오 (FMNH), Algodôr (c. $37^{\circ} 45^{\prime} \mathrm{N},-7^{\circ} 48^{\prime} \mathrm{W}$ ), pitfall traps, 30.0710.09.2003, PC. - SPAIN: 2 우 (NMPC), Catalonia, Deltebre (c. $\left.40^{\circ} 44^{\prime} \mathrm{N},-0^{\circ} 50^{\prime} \mathrm{W}\right), 26.04 .2004, \mathrm{LD} ; 1$ 우 (NMPC), Guardamar del Segura, 28.04.2004, LD; 1 ○, 1 q (NMPC), Besalú, 2.05.2004, LD; $1 O^{7}$ (NMPC), Catalonia, Riumar, 26.04.2004, LD; 1 \% (NMPC), Valensia, Guadalest (c. $\left.38^{\circ} 41^{\prime} \mathrm{N}, 0^{\circ} 11^{\prime} \mathrm{E}\right), 27.04 .2004$, LD; $1{ }^{\prime}$ (NMPC), La Albufera nature reserve, S of Valencia, 30.04.2004, LD; 1 \& (SMFM), Murcia Prov., W of Alhama de Murcia, Sierra de Espuna $\left(37.85601^{\circ} \mathrm{N},-1.49431^{\circ} \mathrm{W}\right)$, c. $691 \mathrm{~m}$ a.s.1., Pinus forest and open pastures, under dry stones, 4.06.2010, SH \& AS. - FRANCE: $1 \sigma^{7}$ (SMFM), Corsica, Col de Verde, c. $1060 \mathrm{~m}$ a.s.1., mixed forest, 9.05.1982, JMS. - ITALY: $2 \mathrm{O}^{7} \mathrm{O}^{7}$ (SMFM), Sicily, c. $2 \mathrm{~km} \mathrm{~W}$ of Castelbuono, stony SE-slope, moist grass beneath grainfields with Fraxinus ornus (under stones), 31.03.1957, HK; 1 O $^{7}$ (SMFM), Sicily, Liparian Island, Ustica Isl., SE-coast, rocky, very dry, a bit grass, stonewalls with hedges of Opuntia (under stones), 6.04.1957, HK; 1 or (SMFM), Sicily, same locality, NE-soast, rocky, cultivated lands, vineyard, grainfileds, stonewalls with Euphorbia and hedges of Opuntia (under stones), 6.04.1957, HK; 1 (SMFM), Sicily, same locality, South slope Mt. Guardia, rocks, dry grass, Pteridium aquilinum, Cystus, Cyisus (under stones), c. 370 m a.s.1., 18.04.1957, HK. — GREECE: $1 \mathrm{O}^{7}$ (SMFM), Crete, nr. Ajil Deka, Gortys, E-slope, 85-100 m a.s.1., scree, bramble, Cirsium, few grass, 9.04.1958, HK.

COMMENTS. A Mediterranean species, known from the Morocco and Iberian Peninsula [Helsdingen, 2014; present data] to Azerbaijan [Logunov, Guseinov, 2002; Marusik et al., 2004], southward to Ivory Coast [Logunov, Azarkina, 2006] and Yemen [Wesołowska, van Harten, 2007].

\section{Phlegra cinereofasciata (Simon, 1868)}

MATERIAL. TURKEY: $10^{7}$ (OUMNH), Tüz Gölu (slopes on NE corner), under stones, 28.07.1956, GL; $10^{7}, 2$ 우 (OUMNH), Van, c. $30 \mathrm{~km}$ of Van Tatvan, at $7500 \mathrm{ft}$ on hillside of Artor Dağ, under stones, 2.09.1956, GL; $7 \sigma^{7} \sigma^{7}, 3$ 3 (OUMNH), Van, c. 75 $\mathrm{km}$ Van Tatvan, under stones and rocks, Pelli Dağ, $7400 \mathrm{ft}$., 4.09.1956, GL; 1 + (NMPC), Çağlayan, c. $24 \mathrm{~km} \mathrm{SE}$ of Erzincan $\left(39^{\circ} 36^{\prime} \mathrm{N}, 39^{\circ} 42^{\prime} \mathrm{E}\right)$, valley above waterfall, 23-24.06.2002, MR; 1 $\mathrm{O}^{\top}, 2$ + + (BMNH), Bazergum, under stones and grass-herb litter, 
23.09.1958, coll.? - CYPRUS: 1 ( (LMNM), Tróodos, Olimpus Mt., c. $1951 \mathrm{~m}$ a.s.1., Juniperus, pine and Whitebeam over, low, scattered herbs, among rocks, 8.09.1997, SJ \& CF

COMMENTS. A Mediterranean species, known from the Iberian Peninsula [Helsdingen, 2014] to the E Caucasus (Azerbaijan) [Azarkina, 2004; Marusik et al., 2004]; the records from Cyprus [present data] lie at the southernmost limit of the species' range. New record for Cyprus.

\section{Phlegra fasciata (Hahn, 1826)}

MATERIAL. SPAIN: $10^{7}$ (NMPC), Catalonia, Mataró (c. 41 ${ }^{\circ} 32^{\prime}$ $\left.\mathrm{N}, 2^{\circ} 27^{\prime} \mathrm{E}\right), 1.05 .2004, \mathrm{LD} ; 3 \mathrm{O}^{7} \mathrm{O}^{\top}$ (NMPC), Besalú, 2.05.2004, LD — ITALY: 1 (SMFM), Torre del, Lago Puccini n. Piza, sand plots, 05.1959, O. Kraus. - GREECE: 1 O7 (LMNM), Lesbos, Stavros $\left(39^{\circ} 02^{\prime} 12^{\prime \prime} \mathrm{N}, 26^{\circ} 16^{\prime} 45^{\prime \prime} \mathrm{E}\right), 158 \mathrm{~m}$ a.s.l., damp, rocky and grazed roadside field with low shrubs among grass, 21.04.1997, SJ; 1 O $^{7}$ (LMNM), Lesbos, Angliki Skala, nr. Krioneri $\left(39^{\circ} 11^{\prime} 35^{\prime \prime} \mathrm{N}\right.$ $26^{\circ} 18^{\prime} 33^{\prime \prime} \mathrm{E}$ ), pine woodland over mixed scrub alongside river, 24.04.1997, SJ; 1 q (LMNM), Lesbos, Skala Kallonis $\left(39^{\circ} 12^{\prime} 23^{\prime \prime} \mathrm{N}\right.$,

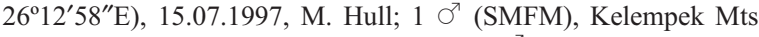
between Avas and Esimi, 05.1968, JMS; $10^{7}$ (SMFM), S coast of Peloponnes, Ligia, beach, dunes and adjacent cultural landscape, 1.06.1998, LM. - CYPRUS: 1 (SMFM), no date and collector. TURKEY: 1 (LMNM), Konya, c. 10 km along Akașehir-Cetince road, Sultan Dağlari, mixed herbs and shrubs among young pine plantation, 19.09.1993, SJ \& CF; 1 क (LMNM), Antalya, Aksal Dağlari, c. $31 \mathrm{~km} \mathrm{~N}$ of Alanya, 19.07.1992, SJ; 1 o (LMNM), Kayseri, Demirkazık, 12-13.06.1993, CF; 1 + (LMNM), İsparta, Kovada Gölü, sparsely vegetated lakeside shingle, 20.06.1993, CF; 1 $\sigma^{7}$ (PCSD), S-shore of Lake Salda, 29.05.1997, RS; 1 O $^{7}$ (NHMW), Namrun, pine forest, 12.05-5.06.1964, FR; $1 \mathrm{O}^{\text {T }}$ (SMFM), W affluent of Sapanca Gölü N of Kurtköy at the road, no date, coll.? ABKHAZIA: 1 (ZMUM), nr. Sukhum, left bank of Kelasuri River (c. $3 \mathrm{~km}$ upstream of its mouth), under stones, 5.06.2004, EM; $1 \mathrm{O}^{7}$ (ZMUM), Sukhum, hand collecting in orchard, 7-9.06.2004, EM.

COMMENTS. A Euro-Siberian temperate-subboreal species, known from the Iberian Peninsula [Helsdingen, 2014] to NW China (Xinjiang), southwards to Afghanistan and N India [Logunov, Marusik, 2000b]. New record for Cyprus.

Phlegra nitidiventris (Lucas, 1846)

MATERIAL. ALGERIA: $10^{7}$ (PCRB), Wilaya Tizi Ouzou, Massif Djurdjura, Tala Gulief, c. $1500 \mathrm{~m}$ a.s.l., pitfall traps in Quercus plusieurs forest, 25.11.1986-29.05.1987, RB; 1 O (PCRB), same locality, c. $1350 \mathrm{~m}$ a.s.l., in open Quercus ilex forest, 1.05.1982, RB; $1 \mathrm{O}^{\top}$ (ZMUM), Wilaya Blida, Chrea, c. 1500 $\mathrm{m}$ a.s.1., mountain grassland, 23.02.1986, RB; $1 \mathrm{O}^{\top}$ (PCRB), Wilaya Blida, Atlas Blidéen, Chrea E., peak Fertasse, c. 1450 m a.s.l., pitfall traps in Cedrus forest, 12.04.1987-9.05.1988, RB; 1 क (ZMUM), Wilaya Blida, Atlas Blidéen, Meurdja, c. 955 m a.s.l., among stones in recent Cedrus plantation, 1.04.1982, RB; 1 (ZMUM), Wilaya Tissemsilt, Massif Ouarsenis, Theniet el Had Djebel Meddad, top, c. 1780 m a.s.l., meadow, 4.05.1989, RB; $10^{2}$ (PCRB), Wilaya Bouira, entrance to Aomar et Kadiria, Wadi Isser, c. $200 \mathrm{~m}$ a.s.l., small plantation of Eucalyptus (in litter), 18.08.1988, RB; $1 O^{2}$ (ZMUM), Wilaya Bouira, Massif Djurdjura, Tikjda, c. 1450 m a.s.l., paifall traos in Ampelodesma grassland, 2-24.04.1982, $\mathrm{RB} ; 2 \mathrm{O}^{7} \mathrm{O}^{7}$ (PCRB), same locality, c. $1500 \mathrm{~m}$ a.s.l., among stones in grassland around hotel, 12.10.1982, RB; 1 ( $\mathrm{PCRB}$ ), Wilaya El Tarf, W of El Kala, S of Bou Malek, Lac Melah, c. 3 m a.s.l., in litter of Quercus suber forest (very close to the lake), 6.04.1982, RB; 1 on, 1 \& (SZMN), Wilaya Tizi Ouzou, forest Akfadou, Lac Agoulmin Abernane, c. $1250 \mathrm{~m}$ a.s.l., among stones along the lake and in litter of the bordering Ouercus faginea forest, 22.04.1982, RB; 2 +P (PCRB), Wilaya Tissemsit, Bou-Caid, N-side of Djebel Ouarsenis, c. $1400 \mathrm{~m}$ a.s.1., in litter of Cedrus forest, 24.03.1988, RB. - PORTUGAL: 2 OP (OUMNH, bottle 2161), Lisbon. TUNISIA: $1 O^{T}$ (SMFM), El Houaria, Macchie with Cistus, Pista- cia and Colycotome, slightly moist sand (under stones), 29.03.1959, HK; 1 O (SMFM), nr. Tunis, 2.03.1961, K.J. Walch; 2 O $^{7}$ (SMFM), c. $2 \mathrm{~km}$ of El Houaria, SW valley, clover, Hieracium, Ulex (sandy soil), 30.03.1959, HK; 1 (SMFM), S of Tunis, Sslope, Cirisium and Cistus, stones with sand (under stones), 29.03.1959, HK.

COMMENTS. A west-Mediterranean species, known from Portugal, Algeria and Tunisia [present data]. The present identification is based on S. Heçiak's illustrations of the types reproduced by Prószyński [2003: figs 562-563]. New record for Portugal.

\section{Phlegra sierrana (Simon, 1868)}

MATERIAL. PORTUGAL: $1 \sigma^{\top}$ (FMNH), Braciais (c. 37 $38^{\prime}$ $\mathrm{N},-7^{\circ} 34^{\prime} \mathrm{W}$ ), pitfall traps, 24.09.2003, PC.

COMMENTS. The Iberian Peninsula only [Helsdingen, 2014; present data]. New record for Portugal.

\section{Plexippoides gestroi (Dalmas, 1920)}

MATERIAL. GREECE: 2 우 (SMFM), Island Kos, nr. Zia, 500-800 m a.s.1., forest and macchia, 29.04.1968, JMS. - TURKEY: 1 ㅇ (PCRS), Mazi Beach, nr. Bodrum, under stone in olive grove, 7.06.1997, ARS; 1 (PCRS), Turbuklu, nr. Bodrum, under stone in dry hillside, 4.06.1997, ARS; 1 क (LMNM), Iccel, c. $2 \mathrm{~km} \mathrm{~S}$ of Çamlidere $\left(36^{\circ} 52^{\prime} 12^{\prime \prime} \mathrm{N}, 34^{\circ} 24^{\prime} 37^{\prime \prime} \mathrm{E}\right)$, rocky disuded quarry at side of road, dominated by Euphorbia, Mullein Cistus and Rubus, 30.06.1995, SJ \& CF; $2 \sigma^{7} \sigma^{7}, 3$ 우 (OUMNH), Urfa, c. $15 \mathrm{~km}$ of Urfa Gayiontep, under stones, 12.09.1956, GL; 2 우 (ZMUM), Antalya, Kemer, pine forest, 50-400 m a.s.1., 23-27.04.2001, KM; $1+$ (PCSD), Pamukkale, ruins of Heiropolis, 20.05.1997, RS; 1 i (NHMW), Kazan, 27.04.1963, FR; 1 (NHMW), Pinus halepensis forest, 9.06.1977, H. Nemenz. - CYPRUS: 1 + (ZMUM), E of Limassol, Mt. Amathus, 5.01.2004, K.G. \& Ya.K. Mikhailov. AZERBAIJAN: 2 우 (MMUM), Naxçivan, Sharur Distr., Akhura $\left(39^{\circ} 34^{\prime} \mathrm{N}, 45^{\circ} 11^{\prime} \mathrm{E}\right), 2.06 .2003, \mathrm{EG}$

COMMENTS. An eastern Mediterranean subboreal species, known from Greece (both mainland and islands) [Helsdingen, 2014] to Azerbaijan [Logunov, Guseinov, 2002; Marusik et al., 2004; present data]. New record for Cyprus. 1872)

Plexippoides flavescens (O. Pickard-Cambridge,

MATERIAL. ISRAEL: $2 O^{7} O^{7}$ (NMPC), Sede Boqer, Negev desert, between Beer Sheva and Mitzpe Ramon, 6-29.05.2003, MR. - IRAN: 1 क (MMUM), Isfahan (c. $32^{\circ} 38^{\prime} \mathrm{N}, 51^{\circ} 39^{\prime} \mathrm{E}$ ), 11.2005, H. Ghahari; $10^{7}$ (MMUM), Lorestan Prov., Kuhdasht (c. $\left.33^{\circ} 32^{\prime} \mathrm{N}, 47^{\circ} 36^{\prime} \mathrm{E}\right), 29.07 .2011, \mathrm{SZ} ; 1$ ㅇ (MMUM), same prov., Khorramabad (c. $33^{\circ} 29^{\prime} \mathrm{N}, 48^{\circ} 21^{\prime} \mathrm{E}$ ), 27.10.2011, SZ; 1 ( (MMUM), same prov., Azna (c. $\left.33^{\circ} 37^{\prime} \mathrm{N}, 48^{\circ} 56^{\prime} \mathrm{E}\right), 9.11 .2011, \mathrm{SZ} ; 1$ 우 (MMUM), Zaghe, 13.10.2012, SZ.

COMMENTS. An eastern Mediterranean - Central Asian subboreal species, known from the Arabian Peninsula and Sinai [Wesołowska, van Harten 2007, 2011] to Turkmenistan and Afghanistan [Logunov, Zamanpoore, 2005].

Plexippus clemens (O. Pickard-Cambridge, 1872) MATERIAL. ISRAEL: $10^{7}$ (NMPC), Sede Boqer, Negev desert, between Beer Sheva and Mitzpe Ramon, 6-29.05.2003, MR.

COMMENTS. Recorded from Libya, Yemen [Wesołowska, van Harten 2007], Israel [Prószyński, 2003; present data], Turkey [Coşar et al., 2014] and Iran [Logunov, 2010]. 
Plexippus devorans (O. Pickard-Cambridge, 1872)

MATERIAL. ALGERIA: $1 \bigcirc^{7}, 5$ 우 (PCRB), Wilaya Laghouat, c. $20 \mathrm{~km} \mathrm{~S}$ of Laghouat, c. $740 \mathrm{~m}$ a.s.l., pitfall traps in Zizyphus stand, 13.01-3.11.1989, RB; 2 우 (PCRB), Wilaya Gardaia, Beni Isguen, c. $525 \mathrm{~m}$ a.s.l., pitfall traps in palm stand, 13 . 01-3.11.1989, RB. - AZERBAIJAN: $1 \mathrm{O}^{\top}$ (ZISP, A.No. 83-1909), between Petropavlovka and Nikolaevka, 09.1909, N. Shavrov.

COMMENTS. A Mediterranean - Central Asian subboreal species, known from Algeria [present data], eastward to Tajikistan and Afghanistan [Logunov, Rakov, 1998: sub P. coccineus; Logunov, Zamanpoore, 2005], and southward to Yemen [Wesołowska, van Harten, 1994: sub P. paykulli]. New record for Algeria.

\section{Plexippus paykulli (Audouin, 1826)}

MATERIAL. ALGERIA: $2 \sigma^{7} O^{7}$ (PCRB), Wilaya Tissemsilt, Theniet el Had, Djebel Meddad, c. $1450 \mathrm{~m}$ a.s.l., under stones in the mixed forest of Cedrus atlanticus and Quercus faginea, 18.05.1989, RB; 1 O (PCRB), Wilaya Bejaia, E. Adekar, along N34, 800 m a.s.1., Quercus ilex forest (in litter), 17.04.1982, RB; 1 $\sigma^{7}$ (PCRB), Wilaya Blida, Chrea, c. $1200 \mathrm{~m}$ a.s.l., among stones in Cedrus forest, 1.11.1985, RB; $4 \bigcirc^{7} \sigma^{7}, 2$ ㅇ (PCRB), Wilaya Alger, Beaulieu, c. $50 \mathrm{~m}$ a.s.l., in garden, no date, RB; 1 + (PCRB), Wilaya Biskra, Tolga, c. $125 \mathrm{~m}$ a.s.l., in litter and between Graminea in palm grove, 10.02.1982, RB. - SPAIN: $1 O^{T}$ (NMPC), Xávia (=Jávea), NP del Mongú, 29.04.2004, LD; 3 O $^{7}, 3$ 오 (NMPC), Guardamar del Segura, 28.04.2004, LD. — GREECE: 1 (LMNM), Vrondados $\left(38^{\circ} 24.95^{\prime} \mathrm{N}, 26^{\circ} 07.88^{\prime} \mathrm{E}\right), 15.09 .1996$, M.J. Taylor; 1 壬 (SMFM), Crete, nr. Canea, 06.1926, CR; $1 \sigma^{T}$ (LMNM), Crete, 09.2002, CF; 3 O $^{7} \sigma^{7}, 2$ ㅇ (SMFM), N Crete, Gouves, 3-4.05.2008, HE \& UK; $1 \sigma^{7}$ (SMFM), N Crete, Analipsi, 5.05.2008, HE \& UK $2 O^{\top} O^{\top}, 1$ ㅇ (SMFM), S Crete, Keratokampos, 7.05.2008, HE \& UK; 1 Or $^{7}$ (SMFM), Crete, Sarhos, nr. Honos Cave, 11.07.2007, HE \& UK; 1 + (SMFM), E Crete, Skopi, 14.05.2007, HE \& UK; $10^{7}, 1$ (SMFM), NE Crete, Mochlos, S Stalis, 6-9.07.2007, HE \& UK; 1 (SMFM), S Crete, Arvi, 12.05.2007, HE \& UK; $10^{77}$ (SMFM), N Crete, Malia, 11.05.2008, HE \& UK; $1 \sigma^{7}$ (NHRS), Crete, Chania Pref., Aghia Marina, indoor, 29.04.1997, TK. - TURKEY: $2 O^{7} \sigma^{7}, 19$ (PCRS), Akyaka, Thomsan Villas, on walls of house, 31.05.1996, ARS; $1 \sigma^{7}$ (PCSD), Dalyan, c. $1 \mathrm{~km} \mathrm{~N}$ of Hotel Gol, 20.05.1997, RS; 1 O$^{7}$, Antalya, Kemer, inside house, 26-26. 04.2001, KM; $10^{7}$ (NHMW), Antalya, 15.05.1965, FR. - TUNISIA: 1 ( (SMFM), Djerba, centre of island, sandy fields, running over ground, 15.04.1959, HK; 1 क (MHNG), c. $13 \mathrm{~km}$ of Zarzis (S de la Tunisie), nr. De 12 Hotel-Club Ornarit, 5.05.1996, PH. EGYPT: 1 + (NHMW), El Mansoura (c. $\left.31^{\circ} 04^{\prime} \mathrm{N}, 31^{\circ} 41^{\prime} \mathrm{E}\right), 8.01$. 1981, HEH; $10^{\prime \prime}, 1$ ㅇ (NHMW), Ras El-Barr (c. $31^{\circ} 31^{\prime} \mathrm{N}, 31^{\circ} 50^{\prime} \mathrm{E}$ ), 08.1982, HEH; 1 o $^{7}, 1$ ㅇ (NHMW), Kafr-El-Sheikh (c. $31^{\circ} 07^{\prime} \mathrm{N}$, $\left.30^{\circ} 57^{\prime} \mathrm{E}\right), 1.12 .1981$, HEH. — ISRAEL: $2 \sigma^{7} \sigma^{7}$ (NMPC), Sede Boqer, Negev desert, between Beer Sheva and Mitzpe Ramon, 629.05.2003, MR; $1 \mathrm{O}^{7}$ (SZMN), Even-Tehuda, 2-15.08.1996, I.I. Lyubechansky; 1 O’ $^{\top}, 2$ ㅇ (ZMUM), Tel-Aviv, Ramatgan, Naupori, 19.04.1995, A. Rasnitsyn.

COMMENTS. A pantropical species, also recorded from the Mediterranean and the Canary Islands [Helsdingen, 2014].

\section{Pseudeuophrys erratica (Walckenaer, 1826)}

MATERIAL. ALGERIA: $1 \overbrace{}^{\lambda}, 1$ (MHNG), Gde Kabylie, Yakouren, c. 730 m a.s.1., 12.05.1988, C. Besuchet, I. Löbl \& D.H Burckhardt. - SLOVENIA: 1 + (SMFM), Rakov Skačjen (?), 18.07.1974, JMS. - TURKEY: 2 Oొ $^{7}$, 3juv. (BMNH), Trabzon, Hamsiköy, c. $1250 \mathrm{~m}$ a.s.1., beaten from low bushherbs on open hillside, 25.08.1958, coll.?; $1 \mathrm{O}^{\top}$ (MHNG), Artvin, sieving of leafand moss-litter in oak \& hornbeam forest, c. $800 \mathrm{~m}$ a.s.1., 7.06.1986, C. Besuchet, I. Löbl \& D.H. Burckhardt. - RUSSIA: $1 \mathrm{O}^{\top}$ (ZISP, A.No. 285), Krasnodar Territory, sloping forest on Dzhubga Mt., 24.05.1911, D. Filatov. - ABKHAZIA: 1 ơ (ZMUM), Novyi Afon, valley of river Psyrtskhe, Carpinus forest, 6.06.2004, EM — GEORGIA: $10^{7}, 2$ juv. (SZMN), Kolchida Reserve, alder for- est, in litter, 14.04.1988, DL; $1 \mathrm{O}^{7}, 1$ ㅇ (ZISP, A.No. 301-470), Kolkhida, N. Chkhugunetch, 500-650 m a.s.l., 3-10.06.1969, EP; 2 우 (ZISP, A.No. 138-915), nr. Batumi, 07-08.1914, K.V. Sukhodolsky; 1 ( SZMN), nr. Tskhaltubo, Satapliisky Reserve, 500-600 m a.s.1., Quercus-Carpinus forest, 15-17.04.1988, DL. - AZERBAIJAN: 1 \& (SZMN), Lenkoran Area, nr. Osakudzha, under bark of logs, 14.07.1983, DL; 1 + (ZMUM), Zakataly reserve (nr. administrative centre), $560 \mathrm{~m}$ a.s.1., KM; $1 \mathrm{O}^{7}$ (SZMN), Astara Distr., between Motlayatat and Bilyasary, Talysh Mts $\left(38^{\circ} 36^{\prime} \mathrm{N}, 48^{\circ} 40^{\prime} \mathrm{E}\right)$, 600-700 m a.s.1., Carpinus-Acer-Quercus forest, 20-22.06.2003, AC.

COMMENTS. A trans-Eurasian temperate species [Logunov, Marusik, 2000b], southwards to N Iran [Logunov et al., 2002].

\section{Pseudeuophrys lanigera (Simon, 1871)}

MATERIAL. SPAIN: $2 O^{7} O^{7}$ (SMFM), SSW of Barselona, 8.10.1953, O. Kraus; $10^{7}$ (NMPC), Barcelona, 25.04.2004, LD; 1 ㅇ (NMPC), Valensia, Monserrat (c. $\left.39^{\circ} 22^{\prime} \mathrm{N}, 0^{\circ} 36^{\prime} \mathrm{W}\right), 1.05 .2004$, LD; $2{\sigma^{7}}^{\prime} \sigma^{\prime \prime}, 1+$ (NMPC), Valensia, Guadalest (c. $\left.38^{\circ} 41^{\prime} \mathrm{N}, 0^{\circ} 11^{\prime} \mathrm{E}\right)$, 27.04.2004, LD; $10^{7}, 1$ ( (NMPC), Xávia (=Jávea), NP del Mongú, 29.04.2004, LD; 4 우 (NMPC), Besalú, 2.05.2004, LD; 1 우 (NMPC), Blanes, 1.05.2004, LD; $1 \mathrm{O}^{\lambda}, 2$ 우 (NMPC), Catalonia, Mataró (c. $\left.41^{\circ} 32^{\prime} \mathrm{N}, 2^{\circ} 27^{\prime} \mathrm{E}\right), 1.05 .2004, \mathrm{LD} ; 1$ $(\mathrm{SMFM})$, Tarragona Prov., N Reus, road from La Riba to Farema $\left(41.31547^{\circ} \mathrm{N}\right.$, $\left.1.10444^{\circ} \mathrm{E}\right), 545 \mathrm{~m}$ a.s.1., under stones in pine forest with evergreen undrestorey, 28.05.2010, SH \& AS. - CROATIA: 1 (ZMTU),

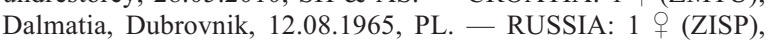
The Crimea (no exact locality), under stones, 20-24.08.1928, K. Min'kova.

COMMENTS. A European - Mediteranean subboreal species, known from the Iberian Peninsula [Helsdingen, 2014] to the E Caucasus (Azerbaijan) [Logunov, Guseinov, 2002].

\section{Pseudeuophrys obsoleta (Simon, 1868)}

MATERIAL. SPAIN: $1 \sigma^{7}$ (NMPC), Montserrat, 1.05.2004, LD. - SLOVENIA: 4 O $^{7} O^{7}$ (ZMTU), Vipava, 11.04.1964, PL. GREECE: $4 \sigma^{\top} \sigma^{\top}, 1$ (SMFM), Kelempek Mts, between Avas and Esimi, 05.1968, JMS; $10^{77}$ (SMFM), Crete, W of Camaris, S-slope, rocky, Phrygana, Liliacea, 6.04.1958, HK. - TURKEY: 1 o (LMNM), Içel, Camliyayla, sparsely vegetated river-bed, 11.06. 1993, SJ \& CF; 1 O $^{7}$ (LMNM), Içel, Tarsus-Çamliyayla, c. 3 km S of Sarıkavak, herb-rich limestone hillside, 11.06.1993, SJ \& CF; 1 ㅇ (LMNM), Konya, Kizilören, 9.07.1992, CF; 1 을 (LMNM), Bağkonak, Sultan Dağlari, under dwarf hillside herbs, 19.09.1993, CF; 10 Oᄋ (ZMUM), Antalya, Kemer, pine forest, $50-400 \mathrm{~m}$ a.s.1., 23-27.04.2001, KM; 2 우 (NMPC), Yakaavsar near Aksu, Dedegol Mts, Pinus forest below Raham Mt., c. 2200 m a.s.1., 9.07.2003, MR; $1 O^{7}$ (NMPC), nr. Pazar, c. $24 \mathrm{~km} \mathrm{~W}$ of Tokat $\left(40^{\circ} 16^{\prime} \mathrm{N}, 36^{\circ}\right.$ $\left.17^{\prime} \mathrm{E}\right)$, Ballica, nr. Mağarasi cave, 29.06-1.07.2002, MR; 1 ㅇ (NMPC), Aksu, valley next to Zindan cave, 6.07.2003, MR; 1 o (NMPC), Yenísarbademli, shore of Beysehir lake, forest steppe with Quercus sp. on corroded limestone, 11.07.2003, MR; 2 우 (ZMTU), Izmir Distr., Yamanlar Daği, c. $1100 \mathrm{~m}$ a.s.l., under stones in the alpine zone, 24.05-30.07.1973, PL \& F. Önder; $1 \sigma^{7}$ (PCSD), nr. Fethiye, above Arpacik, 28.05.1997, RS; 2 우 (PCSD), pool by road above Sultaniye, 22.05.1997, RS; $1 \sigma^{7}$ (NHMW), c. 7 $\mathrm{km}$ E of Ižmit, c. $50 \mathrm{~m}$ a.s.l., bushes on pastures, 25.05.1967, JG, FR \& AR; 1 + (NHMW), c. $30 \mathrm{~km} \mathrm{SW}$ of Samsun, SamsunMerzifon Rd, c. 500 m a.s.l., deciduous forest of Quercus, Cotinus, Ligustrum, Prunus, Rosa, etc., 3.06.1967, JG, FR \& AR; 1 ㅇ (NHMW), Uludağ b. Bursa, c. 950 m a.s.1., 25.05.1968, coll.?; $10^{7}$ (NHMW), Konya, 21.05.1960, FR; $10^{7}$ (NHMW), Akșehir, Sültandağ, 5.05.1960, FR; $1 \sigma^{7}$ (NHMW), Namrun, pine forest, 12.055.06.1964, FR; $1 \mathrm{O}^{7}$ (NHMW), Thetiye, 1964-1965, FR; $10^{7}$ (SMFM), Mugla, c. $7 \mathrm{~km} \mathrm{~W}$ of Fethiye, bridge nr. Yanikla, slope, 6.03.1977, RK. - CYPRUS: 3 우 (PCSD), nr. Cedar valley, on rocks, 29.04.2007, RS. - AZERBAIJAN: $10^{7}$ (SZMN), Lenkoran Area, Apo (c. $\left.38^{\circ} 38^{\prime} \mathrm{N}, 48^{\circ} 47^{\prime} \mathrm{E}\right), 28.05 .2003$, N. Snegovaya. 
COMMENTS. A European - Central Asian subboreal species [Logunov, Marusik, 2000b]. New record for Cyprus.

Pseudeuophrys vafra (Blackwall, 1867)

MATERIAL. ALGERIA: 2 우 (PCRB), Wilaya Alger, Ben Aknoun, $100 \mathrm{~m}$ a.s.l., terrain vague, 18.05.1988, RB; $1 \mathrm{O}^{7}$ (PCRB), Wilaya Setif, Djebel Babor, c. 1800 m a.s.l., pitfall traps in Cedrus forest, 29.06.1988, RB. - SPAIN: 1 \& (NMPC), Salou, 26.04.2004, LD. - ITALY: 1 O $^{7}$ (SMFM), Sardinia, Arzachena, 19.04.1959, Kalimann. - CROATIA: 1 O $^{\prime}, 1$ + (SMFM, 7701), Dalmatia (no exact locality), 05.1938, coll.? - RUSSIA: 1 \% (SZMN), The Crimea, Bakhchisarai, 24-26.06.1993, SV.

COMMENTS. A Mediterranean species, known from the Azores, Iberian Peninsula and Algeria [Helsdingen, 2014; present data] to the Crimea [Kovblyuk, 2004]. New record for Algeria.

\section{4)}

Pseudicius arabicus (Wesołowska et van Harten,

MATERIAL. EGYPT: 1 ( NHMW), El Mansoura (c. $31^{\circ} 04^{\prime} \mathrm{N}$, $\left.31^{\circ} 41^{\prime} \mathrm{E}\right), 8.01 .1981$, HEH.

COMMENTS. The taxonomic status of this species remains poorly resolved. The epigyne and spermathecae of the studied female are identical to those illustrated by Wesołowska \& van Harten [2007: figs 174-177] under the name Pseudicius braunsi Peckham et Peckham, 1903. More importantly, Wesołowska \& van Harten considered the name $P$. braunsi a senior synonym of $P$. arabicus (contra Logunov, Zamanpoore [2005]). However, I am still of the opinion that true $P$. braunsi [sensu Logunov, 1995] and the species reported by Wesołowska \& van Harten [2007] under this name from Yemen are not conspecific. The Yemeni species may belong either to Pseudicius asoroticus Simon, 1908 described from Yemen or to P. tripunctatus Prószyński, 1989 from Saudi Arabia. If the latter assumption is correct, to date $P$. arabicus is known from NE Africa (Egypt and Yemen) and Afghanistan only [Wesołowska, van Harten, 1994; Logunov, Zamanpoore, 2005; present data]. New record for Egypt.

\section{Pseudicius badius (Simon, 1868)}

Figs 104-105.

MATERIAL. IRAN: $1 \sigma^{7}$ (NHMW), [Mazandaran Region], c. $35 \mathrm{~km} \mathrm{~S}$ of Chalus, 1970, K. Bilek.

COMMENTS. A Mediterranean - Central Asian subboreal species, known from Spain [Metzner, 1999; Helsdingen, 2014] to NW Iran [present data]. New record for Iran.

Pseudicius encarpatus (Walckenaer, 1802)

MATERIAL. PORTUGAL: $1 \sigma^{\top}$ (FMNH), Paúl do Boquilobo Biosphere Reserve (c. $\left.39^{\circ} 23^{\prime} \mathrm{N},-8^{\circ} 32^{\prime} \mathrm{W}\right), 11.06 .2002$, PC. TURKEY: $270^{7} O^{7}, 24$ OO (MMUM), Oren (Prov.), cherry orchards, 03.2000, S. Tezcan; $1 \sigma^{7}$ (MMUM), Armutlu, cherry orchards, 03.2000, S. Tezcan.

COMMENTS. A European - Central Asian temperate species, known from the Iberian Peninsula to the S Urals (Orenburg region) and W Turkmenistan [Logunov, Rakov, 1998; Logunov, Marusik, 2000b].
Pseudicius kulczynskii Nosek, 1905

MATERIAL. GREECE: $1 \sigma^{7}$ (MHNG), Island of Rhodes, Lindos, c. $150 \mathrm{~m}$ a.s.1., 17.05.2001, CL. - TURKEY: $1 \mathrm{O}^{7}$ (LMNM), Kayseri, c. $31 \mathrm{~km} \mathrm{~S}$ of Duvalli, rocky outcrop with Euphorbia sp. and mixed scrub, 13.07.1992, SJ \& CF; $1 \sigma^{7}$ (LMNM), Kayseri, Demirkazık, c. 7000' a.s.1., herb-rich alpine pasture, 12-13.06.1993, CF; 1 + (LMNM), İsparta, c. $4 \mathrm{~km} \mathrm{E} \mathrm{of} \mathrm{Egridir,} \mathrm{inside} \mathrm{hotel} \mathrm{on}$ window, 8.07.1992, CF; 2 우 (LMNM), Yozgat $\left(40^{\circ} 01^{\prime} 43^{\prime \prime} \mathrm{N}, 34^{\circ}\right.$ $37^{\prime} 21^{\prime \prime}$ ), Bogazkale, Bashkent Motel, grassy hillside, Populus coppice with Ranunculus dominant, 14.05.1994, SJ \& CF; $10^{7}, 1$ O (ZMTU), Izmir Distr., Yamanlar Daği, c. $1100 \mathrm{~m}$ a.s.l., under stones in the alpine zone, 24.05-30.07.1973, PL \& F. Önder; 3 $\sigma^{7} \sigma^{7}$ (NHMW), Akşehir, Sültandağ, 23-31.05.1960, FR.

COMMENTS. An eastern Mediterranean species, recorded from the North Aegean islands, Turkey and Syria only [Helsdingen, 2014; Prószyński, 2003; Coşar et al., 2014; present data].

Pseudicius picaceus (Simon, 1868)

MATERIAL. GREECE: $1 \sigma^{\top}$ (SMFM), Crete, nr. Canea, 06.1926, CR; 1 O $^{7}$ (SMFM), Crete, Topolia, 06.1926, CR; 3 oㅇ (SMFM), 'Ateropolis', no exact locality and date; $3 \mathrm{O}^{7} \mathrm{O}^{7}, 1$ 9 (SMFM, 4151), Crete, Akrotiri plain, 05.1928, CR; 1 + (SMFM, 1991), Crete, Lakkos, 06.1926, CR; 4 Or $^{7}$ (SMFM, 9301), Crete, Topolia, 06.1926, CR; $4 \mathrm{O}^{7} \mathrm{O}^{7}$ (SMFM), N Crete, Gouves, 1-4.05. 2008, HE \& UK; $2 \sigma^{7} \sigma^{7}$ (SMFM), S Crete, Arvi, 12.05.2007, HE \& UK; 2 O $^{7}$ (SMFM), S Crete, Miliaradon, 11.05.2007, HE \& UK; $1 \sigma^{7}$ (SMFM), SE part, Kalamafka, 14.05.2009, KE; $1 \sigma^{7}$ (SMFM), N Crete, Analipsi, 12.05.2009, KE. - TURKEY: $2 \sigma^{7} \sigma^{7}$ (PCRS), Akyaka, on rocks behind Cinan Beach, 30.05.1996, ARS; $10^{2}$ (PCSD), pool by road above Sultaniye, 22.05.1997, RS; 1 \% (SMFM), TR Kidirak S of Fethiye (Muğla) $\left(36^{\circ} 31^{\prime} \mathrm{N}, 2^{\circ} 08^{\prime} \mathrm{E}\right)$, 24-28.05.1988, RK. - AZERBAIJAN: 1 O (MMUM), Lenkoran Area, Pirasora (c. $38^{\circ} 43^{\prime} \mathrm{N}, 48^{\circ} 23^{\prime} \mathrm{E}$ ), c. $2000 \mathrm{~m}$ a.s.1., 26.05.2003, N.Yu. Snegovaya.

COMMENTS. An eastern Mediterranean species, known from Italy, throughout Greece and Turkey, to Azerbaijan [Marusik et al., 2004; Helsdingen, 2014; Danişman et al., 2012; present data].

Pseudicius spasskyi (Andreeva, Heçiak et Prószyński, 1984)

MATERIAL. AZERBAIJAN: $1 \sigma^{7}$ (ZISP), nr. Baku, 5.10. 1989, PD

COMMENTS. A Central Asian (Turanian) species, known from Azerbaijan to Tajikistan [Logunov, Rakov, 1998; Logunov, Guseinov, 2002].

Pseudicius spinifer (O. Pickard-Cambridge, 1872) MATERIAL. EGYPT: 2 ㅇ (SMFM), Luxor, island on Nile, bamboo, 17.11.1996, PJ.

COMMENTS. A few localities from Sudan to Syria [WSC, 2015].

\section{Pseudicius tamaricis Simon, 1885}

MATERIAL. TUNISIA: $2 \sigma^{7} \sigma^{7}, 1$ i (ZMUM), Beja, Oued Zarga, 12.09.1985, coll.? - EGYPT: 2 O $^{7} \sigma^{7}$ (SMFM), Luxor, nr. the airport, on bark of trees, 15.11.1996, PJ.

COMMENTS. A Mediterranean species, known from Morocco and the Iberian Penisula to Israel, southward to Saudi Arabia [Prószyński, 2003].

Pseudicius vankeeri Metzner, 1999

MATERIAL: CYPRUS: $1+$ (PCDM), Agridi nr. Dali, in garden, April 2010, DMC; $10^{7}$ (PCDM), same locality, bank of dried out river, 28.04.2013, DMC.

COMMENTS: An eastern Mediterranean species, known from the Dodecanese islands (Greece), Cyprus, 

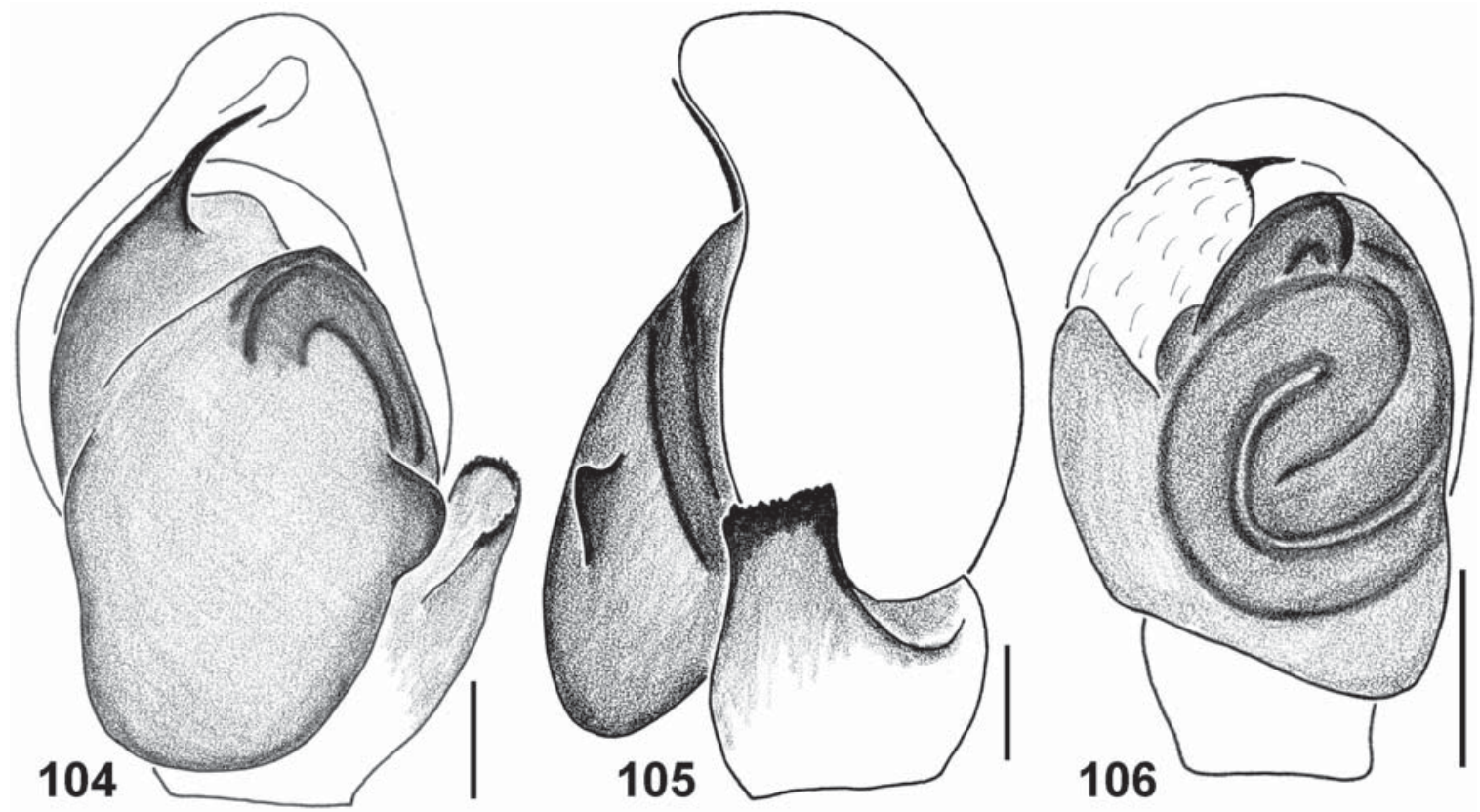

Figs 104-106. Male palps of Pseudicius badius (Simon, 1868) (104-105; Iran, Chalus) and Talavera milleri (Brignoli, 1983) (106; Portugal, Planalto de S. Mamede): 104, 106 - ventral view; 105 — ditto, retrolateral view. Scale bars: $0.1 \mathrm{~mm}$.

Рис. 104-106. Пальпы самцов Pseudicius badius (Simon, 1868) (104-105; Иран, Чалус) и Talavera milleri (Brignoli, 1983) (106; Португалия, Планалту де С. Мамеде): 104, 106 - вид снизу; 105 - тоже, сзади-латерально. Масштаб 0,1 мм.

Turkey and Israel [Logunov, 2009b; present data]. New record for Cyprus.

Saitis ariadneae Logunov, 2001

MATERIAL. GREECE: 1 + (SMFM), Crete (W part), E of Kissamos Castelli, seashore, sandy, thin vegetation with Hordeum murinum, small Euphorbia, 18.04.1958, HK. 2014].

COMMENTS. Restricted to Crete [Helsdingen,

Saitis barbipes (Simon, 1868)

MATERIAL. SPAIN: 1 † (NMPC), Cataluna, Besalú (c. $42^{\circ}$ $12^{\prime} \mathrm{N}, 2^{\circ} 42^{\prime} \mathrm{E}$ ), 25.04.2004, LD; 19 (NMPC), Montserrat, 1.05.2004, LD. - FRANCE: $10^{7}, 4$ 우 (SMFM), Dep. Alpes-Maritimes, Col de Turini, c. $50 \mathrm{~km}$ NNE of Nice, $1500-1650 \mathrm{~m}$ a.s.1., 18-19.09. 1982, JMS; 1 (SMFM), Corsica, Col de Verde, c. 1060 m a.s.l., mixed forest, 9.05.1982, JMS.

COMMENTS. A European species, common in the northern half of the Mediterranean, eastward to the Balkans [Helsdingen, 2014].

Saitis graeca Kulczyński, 1905

MATERIAL. GREECE: 1 क (SMFM), Epirus, Pindos Mts, street between Karpenisi and Agrinio $\left(38^{\circ} 48^{\prime} 3.8^{\prime \prime} \mathrm{N}, 21^{\circ} 29^{\prime} 55.5^{\prime \prime} \mathrm{E}\right)$, c. $746 \mathrm{~m}$ a.s.1., confireous forest (sieving and from under stones), 28.03.2006, AS; 1 + (SMFM), Leukas Island, waterfalls c. $3 \mathrm{~km} \mathrm{~W}$ of Nidri, valley before canyon $\left(38^{\circ} 43^{\prime} 27.8^{\prime \prime} \mathrm{N}, 20^{\circ} 41^{\prime} 13.1^{\prime \prime} \mathrm{E}\right)$, c $200 \mathrm{~m}$ a.s.1., sieved out of evegreen oak's leaves, 27.03.2006, AS 209 (SMFM), Leukas, W of Frini (=Phryni, W of Levkada) $\left(38^{\circ}\right.$ $49^{\prime} 55.8^{\prime \prime} \mathrm{N}, 20^{\circ} 39^{\prime} 59.7^{\prime \prime} \mathrm{E}$ ), c. $10 \mathrm{~m}$ a.s.1., coniferous, sieved out of wet leaf litter nr. rock, 27.03.2006, AS; $10^{7}, 1$ (SMFM), Korfu SW Gouvia, Danilia W Kontokali (39 $\left.37^{\prime} 44.3^{\prime \prime} \mathrm{N}, 19^{\circ} 50^{\prime} 32.4^{\prime \prime} \mathrm{E}\right)$ c. $80 \mathrm{~m}$ a.s.1., oak forest with many open cogs, under stones caught at night, 23.03.2006, AS; $80^{7} \sigma^{7}, 8$ ㅇ (SMFM), Korfu, between Acharavi and Portes $\left(39^{\circ} 48^{\prime} 7.5^{\prime \prime} \mathrm{N}, 19^{\circ} 50^{\prime} 33.1^{\prime \prime} \mathrm{E}\right)$, c. $30 \mathrm{~m}$ a.s.1., Quercus forest (sieving, under wood and stones), 25.03.2006, AS.
COMMENTS. Recorded from Greece (mainland) and Bulgaria only [Helsdingen, 2014].

Saitis sengleti (Metzner, 1999)

MATERIAL. GREECE: 2 Po (SMFM), Crete, Topolia, 06.1926, CR; 2 오 (SMFM), Crete, nr. Canea, 06.1926, CR; 1 (SMFM), Crete, nr. Caripas $\left(35^{\circ} 27^{\prime} 26,0^{\prime \prime} \mathrm{N}, 23^{\circ} 59^{\prime} 36,5^{\prime \prime} \mathrm{E}\right), 123 \mathrm{~m}$ a.s.l., bed of a stream with plane trees, under stones, 27.03.2007, AS; 1 \% (SMFM), Crete, Hrissos Kalitissas ( $\left.35^{\circ} 19^{\prime} 11,6^{\prime \prime} \mathrm{N}, 23^{\circ} 32^{\prime} 56^{\prime \prime} \mathrm{E}\right), 24$ $\mathrm{m}$ a.s.1., macchia in pine forest, under stones, 24.03.2007, AS; 2 $\sigma^{7} \sigma^{7}\left(\right.$ LMNM), Chios, Kallimasia $\left(38^{\circ} 18^{\prime} 01^{\prime \prime} \mathrm{N}, 26^{\circ} 05^{\prime} 38^{\prime \prime} \mathrm{E}\right)$, c. $318 \mathrm{~m}$ a.s.l., flower meadow, 25.04.1997, SJ \& CF; ㅇ (SMFM), Crete, Lassithi plateau, Plati, 15.05.2011, UK \& HE.

COMMENTS. Recorded from Greece (mainland, Chios and Crete) only [Helsdingen, 2014; present data].

Saitis taurica Kulczyński, 1905

MATERIAL. GREECE: $2 \mathrm{O}^{7} \mathrm{O}^{7}$ (SMFM), Delfi, under Castalic Source, 23.04.1968, JMS; $10^{7}, 2$ 우 (SMFM), NE hillside of Ossa Mts, above Omolion, 500 m a.s.l., 20.04.1968, JMS; 3 우 (SMFM), Crete, Topolia, 06.1926, CR; 2 of (PCRG), Thasos, Ipsarion (c. $40^{\circ} 39^{\prime} \mathrm{N}, 24^{\circ} 40^{\prime} \mathrm{E}$ ), c. $820 \mathrm{~m}$ a.s.1., 27.05.2003, AF; 1 (PCRG), Thasos, Thasos valley plain woodland $\left(\right.$ c. $\left.40^{\circ} 39^{\prime} \mathrm{N}, 24^{\circ} 40^{\prime} \mathrm{E}\right)$, c. $120 \mathrm{~m}$ a.s.1., 25.05.2003, AF; $1 \sigma^{7}, 2$ 오 (ZMTU), Rhodos, Petaloudes, in litter of deciduous forest, 22.05.1973, PL. - TURKEY: $1 \sigma^{7}, 1$ (PCRS), Old Yalikavak, nr. Bodrum, under stone in grassland, 30.05.1997, ARS; 2 우 (PCRS), W. Turkey, Selale waterfalls, under stone along stream, 6.06.1996, ARS; 1 क (PCRS), Akyaka, rocks on grass edge of river Akyaka, 3.06.1996, ARS; 2 $\mathrm{O}^{7} \mathrm{O}^{7}, 2$ 우 (NHMW), Hereke, c. $23 \mathrm{~km}$ W of Ižmit, 50-100 m a.s.1., olive plantation (under stones and in grass), 25.05.1967, JG, FR \& AR; $1 \sigma^{7}$ (NHMW), Kuşadası (SE border), 11-26.05.1969, F. Spitzenberger; $3 O^{7} O^{7}, 6$ $6 \bigcirc$ (ZMTU), Izmir Distr., Yamanlar Daği, grassy slope by a mountain brook in forest, 4.05-30.07.1973, PL \& F. Önder.

COMMENTS. An eastern Mediterranean species, known from Greece (both mainland and islands), 
throughout Bulgaria and Turkey [e.g., Uyar, Uğurtaş, 2012; Helsdingen, 2014], to the Crimea [Kovblyuk, 2004; present data].

Salticus iteacus Metzner, 1999

MATERIAL. GREECE: 1 + (SMFM), Delfi, under Castalic Source, 23.04.1968, JMS.

COMMENTS. Two localities in Greece only [Metzner, 1999; present data].

\section{Salticus mutabilis Lucas, 1846}

MATERIAL. MOROCCO: 1 ( (SMFM, 4138), Casablanca, no date and coll. - SPAIN: $3 \sigma^{7} \sigma^{7}$ (NMPC), Barcelona, 25.04.2004, LD; $1 \sigma^{7}$ (NMPC), Denia, 29.04.2004, LD. - ALGERIA: 1 (PCRB), Wilaya Boumerdes, Bordj el Bari, $20 \mathrm{~m}$ a.s.1., garden, in litter of Casuarina equisetifolia, 25.04.1982, RB. - MALTA: 3 $\sigma^{7} \sigma^{7}$ (MHNG), Island of Malta, Buskett Gardens, Verdala Palace, on the bark of Pinus halepensis, 17.05.1974, V. Mahnert. GREECE: 1 o (SMFM), Crete, nr. Canea, 06.1926, CR. - TURKEY: 1 + (SMFM), Uluabta Gölü nr. Apolyont/Apollonia, no date, coll.? - EGYPT: 1 ( NHMW), Ras El-Barr (c. $\left.31^{\circ} 31^{\prime} \mathrm{N}, 31^{\circ} 50^{\prime} \mathrm{E}\right)$ 1.08.1981, A. Shehala. - ABKHAZIA: $10^{7}$ (ZMUM), nr. Sukhum, left bank of Kelasuri River (2-3 km upstream of its mouth), 5.06.2004, EM. - GEORGIA: 1 O $^{7}$ (SZMN), c. $20 \mathrm{~km} \mathrm{E} \mathrm{of} \mathrm{Poti,}$ Kolkhida Reserve, 13.04.1988, DL.

COMMENTS. A European - Mediterranean subboreal species, known from the Azores and Morocco [Helsdingem 2012; present data] to Azerbaijan [Logunov, Guseinov, 2002]. The record from Argentina [Mello-Leitão, 1944] was likely based on a misidentification. New records for Morocco and Egypt.

\section{Salticus noordami Metzner, 1999}

MATERIAL. CYPRUS: 1 (PCSD), Akamas Peninsula, nr. Agios Minas, 3.05.2007, R.G. Snazell; 2 우 (PCMA), Akamas, 3.05.2007, MA; 1 (PCRS), Troodos Mts, Panaghia Tow as in on church, picnic site, on wall 7.05.2007, coll.?; 1 (PCRS), Lumpia, Lumpia Dah, on wall, 9.05.2013, coll.?; $1 \sigma^{7}$ (PCRS), Troodos Mts, Pano Platres, on stone wall, 6.05.2007, coll.?

COMMENTS. An eastern Mediterranean species, known from North Aegean islands (Lesbos) to central Iran [Logunov, 2009b; present data]. New record for Cyprus.

\section{Salticus propinquus Lukas, 1846}

MATERIAL. ALGERIA: $1 \sigma^{7}$ (PCRB), Wilaya El Tarf, El Kala, W bank of Lac Tonga, c. $10 \mathrm{~m}$ a.s.l., wet meadows along lake, 27.03.1988, RB; 1 (PCRB), Wilaya Bejaia, E of the mouth of Wadi Daas, c. $5 \mathrm{~m}$ a.s.l., under stones and on bushes on the shore, 22.05.1988, RB. - ITALY: 1 क (SMFM), Sicily, E of Ficuzza, edge of a path, thorny, dry (under large stones), c. $900 \mathrm{~m}$ a.s.1., 10.04.1957, HK. - GREECE: 1 \% (SMFM), Crete, W of Camaris, 600-800 m a.s.l., slope, rocky, Euphorbia, Liliacea, 6.04.1958, HK. - CYPRUS: 1 ( (PCMA), Pissouri, 24.03.1998, MA. - JORDAN: 1 ( MHNG), E Jordan, Dehbeen b. Jerash, 11.03.1968, J. \& S. Klapperich. - SYRIA: $20^{7} \sigma^{7}, 2$ 우 (NMPC), NW Syria, Mashtal Helu nr. Homs, 30.03-3.04.2001, MR; 1 \% (NMPC), NW Syria, Aln al Zara nr. Idlib, canyon of Oronthes, 28.03.2001, MR

COMMENTS. A Mediterranean species, known from the Iberian Peninsula [Helsdingen, 2014] to the Near East [Prószyński, 2003; present data]. New records for Syria and Cyprus.

Salticus scenicus (Clerck, 1757)

MATERIAL. SPAIN: 2 Oᄋ (MHNG), Zamora, nr. Sandin de Carballeda, 17.07.1975, PH. — RUSSIA: $2 \sigma^{7} \sigma^{7}$ (ZMUM), North
Ossetia, Tchei Vil., Tchei forestry, 1600-2500 m a.s.1., 7.0620.07.1981, S.K. Alexeev.

COMMENTS. A Holarctic temperate species [Logunov, Marusik, 2000b].

Salticus tricinctus (C.L. Koch, 1846)

Figs 107-113.

Calliethera tricincta C.L. Koch, 1846: 50, fig. 1117 (D ${ }^{7}$; holotype $\sigma^{7}$ from the Museum für Naturkunde, Berlin, Germany; examined, Figs 107-108).

Salticus marenzelleri Nosek, 1905: 143, fig. 25 (Dơ일 lectotype $\sigma^{7}$ from Naturhistorisches Museum, Wien, Austria; examined, Figs 109-110). Syn.n.

TYPES: Holotype $\sigma^{7}$ of Calliethera tricincta (Museum für Naturkunde; ZMB 1550, side preparation; Figs 107-108) from [Uzbekistan], Buchara. - Lectotype $\sigma^{7}$ and paralectotype $q$ of Salticus marenzelleri (NHMW, 13825-I; Figs 109-110, 112-113) from Turkey; no data lable is enclosed, but according to Nosek [1905: p. $119 \& 145]$ the type series $\left(1 \sigma^{\gamma}, 1\right.$ \%) was collected from near Ilgün in 9-10 May [1902, A. Penther col.].

MATERIAL. TURKEY: $1 \sigma^{\prime}$ (NMPC), Cağlayan $\left(39^{\circ} 36^{\prime} \mathrm{N}\right.$, $\left.39^{\circ} 42^{\prime} \mathrm{E}\right)$, c. $24 \mathrm{~km}$ SE of Erzincan, valley above waterfall, 2324.06.2002, MR. - ARMENIA: $2 \sigma^{\top} \sigma^{7}, 8$ 우 (ZISP, A.No. $317-$ 09), Yerevan Area, 'station Negram' (unknown locality), 19.05. 1909, N. Bryansky; 1 + (SZMN), nr. Sevan town, under stones, 31.07.1983, DL. - AZERBAIJAN: 1 ( (ZISP, A.No. 273-1909), Lerik, 17.05.1909, A. Kirichenko; $1 \sigma^{7}$ (ZMUM), c. $60 \mathrm{~km} \mathrm{SW}$ of Baku, nr. Gobustan, Gobustan Reserve, 7.05.1989, PD; 2 우 (ZMUM; earlier identified by PD as S. scenicus), Lerik Area, nr. Gosmalyan, $1300 \mathrm{~m}$ a.s.1., 18.06.1985, PD; 2 우 (ZMUM), Gyandzha, nr. Khalar, $600 \mathrm{~m}$ a.s.1., 4.07.1988, PD; $1 \sigma^{7}$ (ZMUM; earlier identified by PD as S. scenicus), Lerik Area, nr, Digyakh, $1600 \mathrm{~m}$ a.s.1., 15.05.1985, PD; 1 ๆ (SZMN), c. $6 \mathrm{~km} \mathrm{~W}$ of Divichi, 28.05.1988, PD; 1 \& (ZMUM; earlier identified by PD as S. scenicus), Khanlar, near water, 19.08. 1986, PD. - RUSSIA: 1 \% (ZMUM), Daghestan, nr. Derbent, 20.05.1989, PD; $2 \sigma^{\top} \sigma^{\top}$ (ZMUM), Astrakhan' Region, Lishansk Distr., E of Basinskaya (c. $46^{\circ} 07^{\prime} 55^{\prime \prime} \mathrm{N}$, $\left.47^{\circ} 13^{\prime} 05^{\prime \prime} \mathrm{E}\right), 18.05 .2012$, AG. - UZBEKISTAN: 1 (MMUM), Surkhandariya Region, Termez Distr., W of shore of Uchkyzyl Reservoir, c. $1.5 \mathrm{~km}$ E of Kaltarkhama $\left(137^{\circ} 20^{\prime} 52^{\prime \prime} \mathrm{N}, 67^{\circ} 12^{\prime} 30^{\prime \prime} \mathrm{E}\right)$, $325 \mathrm{~m}$ a.s.1., 28.04.2002, A.V. Gromov; 1 9 (MMUM), Surkhandariya Region, Termez Distr., Kattakum sands, c. $1.5 \mathrm{~km}$ E of Echkyzyl $\left(137^{\circ} 20^{\prime} 35^{\prime \prime} \mathrm{N}, 67^{\circ} 12^{\prime} 29^{\prime \prime} \mathrm{E}\right), 315 \mathrm{~m}$ a.s.1., 29.04.2002, A.V. Gromov. See also in Logunov \& Rakov [1998].

COMMENTS. An eastern Mediteranean - Central Asian species, known from Turkey [Nosek, 1905: sub S. marenzelleri; present data], throughout the Caucasus, Turkmenistan and southern Kazakhstan [Logunov, Rakov, 1998: map 10; Logunov et al., 2002; Marusik et al., 2004; present data] to Afghanistan [Logunov, Zamanpoore, 2005].

Re-examination of the type series $\left(\sigma^{7}+\right)$ of Salticus marenzelleri (Figs 107-108) and the $\sigma^{\top}$ holotype of Calliethera tricincta (Figs 109-110, 112-113) in comparison with numerous voucher specimens from Turkey (Fig. 111) and Central Asia has shown that all the examined specimens have got the identical copulatory organs in both sexes. The angle of the lateral bend of the embolus in S. tricinctus is the only slightly variable character; cf. Figs 109, 111 and fig. 4a in Logunov [1992]. Therefore it is safe to conclude that $S$. marenzelleri is to be considered a junior synonym of S. tricinctus described originally from Bukhara, Uzbekistan [Koch, 1846: sub Calliethera t.; Logunov, 1992]. 




Figs 107-113. Copulatory organs of Salticus tricinctus (C.L. Koch, 1846) (107-108 - o' holotype of Calliethera tricincta; 109-110, 112-113 - $\sigma^{7}$ lectotype and 9 paralectotype of Salticus marenzelleri Nosek, 1905; 111 — Turkey, Cağlayan): 107 - male palp, retrolateral view; 108 - ditto, prolateral view; 109, 111 - ditto, ventral view; 110 - male palpal tibial apophysis, retrolateral view; 112 - epigyne, ventral view; 113 - spermathecae, dorsal view. Scale bars: $0.1 \mathrm{~mm}$.

Рис. 107-113. Копулятивные органы Salticus tricinctus (C.L. Koch, 1846) (107-108 — О7 голотип Calliethera tricincta; 109-110, 112-113 - О лектотип и + паралектотип Salticus marenzelleri Nosek, 1905; 111 — Турция, Каглаян): 107 — пальпа самца, вид сзади-латерально; 108 - тоже, вид спереди-латерально; 109, 111 — тоже, вид снизу; 110 — тибиальный отросток пальпы самца; 112 - эпигина, вид снизу; 113 - сперматека, вид сверху. Масштаб 0,1 мм. 
Salticus zebraneus (C.L. Koch, 1837)

MATERIAL. PORTUGAL: 1 (FMNH), Paúl do Boquilobo Biosphere Reserve (c. $39^{\circ} 23^{\prime} \mathrm{N},-8^{\circ} 32^{\prime} \mathrm{W}$ ), 28.08.2002, PC. SPAIN: $3 \mathrm{O}^{7} \mathrm{O}^{\top}$ (MVHN), Valencia, Devesa del Saler, dunas with Pinus halepensis, 20.05.2004, DD; $10 \mathrm{O}^{7} \mathrm{O}^{7}$ (NMPC), the nature reserve of La Albufera, S of the city of Valencia, 30.04.2004, LD; 1 o (NMPC), Barcelona, 25.04.2004, LD; $10^{7}$ (NMPC), Catalonia, Mataró (c. $41^{\circ} 32^{\prime} \mathrm{N}, 2^{\circ} 27^{\prime} \mathrm{E}$ ), 1.05.2004, LD. - GREECE: $20^{7} \sigma^{\prime \prime}$ (SMFM), N Crete, Gouves, 1.05.2008, HE \& UK; $1 O^{\top}$ (SMFM), C Crete, Magarikari, 7.05.2009, KE \& HE; 1 o (MHNG), Crete, Lassithi, W of Sitia, Faneromeni Peninsula, c. 5 m a.s.1., 6.05.1985, $\mathrm{BH} ; 1$ (MHNG), Crete, Lassithi, along the road Lasithi-Irakleon, junction to Krasi, c. 570 m a.s.l., 7.05.1985, CL; 1 O $^{7}$ (MHNG), Cyclades, Iraklia (island S of Naxos), Aghio Georgios, rocks on the shore covered 'phrygana' vegetation, 15-20 m a.s.1., 10.05.1985, CL; 1 ( (MHNG), Naxos, above Filoti, foothill of Mt. Zeus, c. 600 $\mathrm{m}$ a.s.1., 14.05.1985, CL; 1 ( ) (SMFM), NW coast of Peloponnes, thermal spring of Loutra Killini, 8.06.1998, LM; 1 \% (SMFM), S coast of Peloponnes, Foinikous, cultural lands, 4.06.1998, LM. RUSSIA: 1 + (ZISP), Krasnodar, sweeping over trees, 25.07.1982, coll.?

COMMENTS. A European temperate species [Logunov, Marusik, 2000b].

Sitticus ammophilus (Thorell, 1875)

MATERIAL. TURKEY: 1 ( NMPC), Tunceli (c. $39^{\circ} 06^{\prime} \mathrm{N}$, 39 $9^{\circ} 3^{\prime} \mathrm{E}$ ), Munsur river, 22.06.2002, MR. - AZERBAIJAN: 1 o' (MMUM), Lenkoran Area, Apo (c. $\left.38^{\circ} 38^{\prime} \mathrm{N}, 48^{\circ} 47^{\prime} \mathrm{E}\right)$, 8.05.2003, EG; 1 O , 1 क (MMUM), 1 O (SZMN), Absheron, nr. Qanlı göl (lake) (c. $\left.40^{\circ} 22^{\prime} \mathrm{N}, 49^{\circ} 49^{\prime} \mathrm{E}\right), 20.05 .2003, \mathrm{EG}$.

COMMENTS. An eastern Mediterranean - Central Asian species recorded to date from Turkey and southern Ukraine [Kovblyuk, 2004; Topçu et al., 2009; Coşar et al., 2014; present data], throughout northern Iran [Logunov et al., 2002] and the Caucasus (Naxçivan) [Marusik et al., 2004] to Turkmenistan [Wesołowska, 1996].

Sitticus atricapillus (Simon, 1882)

MATERIAL. AUSTRIA: $10^{7}, 1$ ( (OUMNH), Tyrol, Cortina g'Ampezz, 1900, G. Nicholson. - TURKEY: 1 o (OUMNH), Bitlis, Nemsut Dağ, c. $2750 \mathrm{~m}$ a.s.l., lake in garden (under stones), 12.08.1956, GL.

COMMENTS. A south-European species restricted to mountainous regions, known from France to Turkey [Kronestedt, Logunov, 2003; present data]. New record for Turkey.

Sitticus damini (Chyzer, 1891)

MATERIAL. ABKHAZIA: 1 क (ZMUM), nr. Sukhum, beach nr. the mouth of Kelasuri River, under stones, 7-9.06.2004, EM

COMMENTS. An eastern Mediterranean species, recorded from Croatia [Helsdingen, 2014] to Abkhazia [present data].

Sitticus distinguendus (Simon, 1868)

MATERIAL. GREECE: $1 \mathrm{O}^{\text {(7 }}$ (LMNM), Lesbos, Vatera $\left(39^{\circ} 01^{\prime}\right.$ 13"N, 2610'53"E), 20.04.1997, SJ. - TURKEY: 1 ㅇ (ZMTU), Izmir Distr., Yozgat Distr., Galatli, wet grassy limestone slope, 11.09.1971, PL; $1 \sigma^{7}$ (PCSD), nr. Dalyan, by river at Caunos, 23.05.1997, RS; 1 O $^{7}$ (NHMW), c. $30 \mathrm{~km} \mathrm{SW}$ of Sivas, gypsum landscape, 6.06.1967, JG, FR \& AR; $1 \sigma^{7}$ (NHMW), Akşehir, Sültandağ, 5-27.05.1960, FR; 1 \& (LMNM), Silifke dunes, Mersin, sand dune system with scattered scrub up to $400 \mathrm{~m}$ from sea, 17.07.1992, SJ \& CF; 1 \& (LMNM), İsparta, c. 4 km E of Egridir, inside hotel on window, 8.07.1992, CF; 1 क (LNMC), Konya, Akșehir, above Dağ Otel, mixed scrub, herbs and grasses on northfacing limestone slope, 19.09.1993, SJ \& CF; $2 \sigma^{7} \sigma^{7}$ (LMNM),
Kayseri, Sultansazligi $\left(38^{\circ} 14^{\prime} 36^{\prime \prime} \mathrm{N}, 35^{\circ} 12^{\prime} 08^{\prime \prime} \mathrm{E}\right)$, degraded edge of marsh, 11.05.1994, SJ \& CF. - AZERBAIJAN: 1 (ZISP, A.No. 360-1933), Naxçivan, Ordubad Distr., nr. Bilav, sandy beach of Gelyan-chai, 27.06.1933, D. Znoiko.

COMMENTS. A trans-Eurasian temperate species [Logunov, Marusik, 2000b].

The recent record of Sitticus avocator (O. PickardCambridge, 1885) from Turkey [Danişman et al., 2012] is mistaken and should be referred to $S$. distinguendus, as the former species is known to be restricted to the SE montane region of Central Asia only (see Logunov $\&$ Marusik [2000a] for the discussion and justification).

Sitticus dzieduszyckii (L. Koch, 1870)

MATERIAL. RUSSIA: 1 (ZISP), Krasnodar Territory, hills nr. Armavir, under stones, 21.04.1911, D. Volnukhin; $1 \sigma^{7}$ (ZISP, A.No. 38), same territory, Peredovaya station, sweeping on the bank of Urup river, 14.07.1920, K. Korol'kova.

COMMENTS. A Euro-Siberian Asian subboreal species, known from France to the Middle Urals [Logunov, Marusik, 2000b].

Sitticus floricola (C.L. Koch, 1837)

MATERIAL. GEORGIA: $1 \mathrm{O}^{\top}$ (ZISP), Kolkhida, Danimarauli, meadow, $1700 \mathrm{~m}$ a.s.1., 26.06.1969, EP.

COMMENTS. A trans-Eurasian temperate species [Logunov, Marusik, 2000b].

Sitticus inexpectus Logunov et Kronestedt, 1997

MATERIAL. GREECE: $1 \mathrm{O}^{\top}$ (ZMTU), Lagos, wet shore meadow with Juncus, Tamarindus, Rubus, etc., 26.09.1971, PL; 1 우 (SMFM), Thrakien, Porto Lago, Ewru, 1.04.1977, RK. - TURKEY: 2 ㅇ (NMPC), Sivas Prov., Nr. Demiryurt, Tödürge Gölü (lake), c. $13 \mathrm{~km} \mathrm{~W}$ of Zara $\left(39^{\circ} 53^{\prime} \mathrm{N}, 37^{\circ} 37^{\prime} \mathrm{E}\right), 25-28.06 .2002$, MR; 19 (NHMW), c. $8 \mathrm{~km}$ SW of Ulubey, $950 \mathrm{~m}$ a.s.l., nr. Ordü, 5.06.1972, coll.? - AZERBAIJAN: 3 우 (SZMN), Lenkoran Area, Liman (c. $\left.38^{\circ} 50^{\prime} \mathrm{N}, 48^{\circ} 49^{\prime} \mathrm{E}\right), 22.05 .2003$, EG; $10^{\prime}$ (SZMN), Khyzy Distr., c. $12 \mathrm{~km} \mathrm{~W}$ of Kilyazi (c. $40^{\circ} 52^{\prime} \mathrm{N}, 49^{\circ} 12^{\prime} \mathrm{E}$ ), 7.06.2003, EG.

COMMENTS. A European - Central Asian temperate species, known from Italy and the UK to Kyrgyzstan [Logunov, Kronestedt, 1997; Helsdingen, 2014; Ijland, Helsdingen, 2014]. Some of the European records of $S$. rupicola may actually belong to this species; see Logunov \& Kronestedt [1997] for the diagnoses of both species. New record for Turkey.

Sitticus pubescens (Fabricius, 1775)

MATERIAL. MOROCCO: $1 \sigma^{7}$ (SMFM, 4141; $\sigma^{7}$ palp only), Tez, 23.05.1930, coll.? - SPAIN: 2 O$^{7} \sigma^{7}$ (MVHN), Valencia, Alicante, Vall d'Ebo, Exterior Avenc Ample, 25.05.2005, SM.

COMMENTS. A European - Central Asian temperate species [Logunov, Marusik, 2000b]. New record for Morocco.

Sitticus relictarius Logunov, 1998

MATERIAL. GEORGIA: $10^{7}$ (ZISP), Kolkhida, Danimarauli, on wooden wall, $1700 \mathrm{~m}$ a.s.1., 26.06.1969, EP

COMMENTSA Caucasian species, known from the the northern Caucasus, Georgia, Azerbaijan and NW Iran [Logunov, 1998b; present data].

Sitticus rupicola (C.L. Koch, 1837)

MATERIAL. ROMANIA: $1 \sigma^{7}, 1$ ( NMPC), Bihor-zona Padiš, 15-21.07.2001, MR. 
COMMENTS. A European species [Helsdingen, 2014], of which distribution requires further clarification, as some of the earlier records could actually belong to the closely related species, $S$. inexpectus: viz., the records from Central Asia (see Logunov \& Kronestedt [1997] for further discussion).

\section{8)}

Sitticus saltator (O. Pickard-Cambridge in Simon,

MATERIAL. TURKEY: 1 (NHMW), [Bursa Prov.], Uludağ, 5.10.1955, H. Nemenz.

COMMENTS. A Euro-Siberian temperate species, known from the Iberian Peninsula [Helsdingen, 2014] to Transbaikalia [Logunov, Marusik, 2000b]. New record for Turkey.

Sitticus zimmermanni (Simon, 1877)

MATERIAL. TURKEY: $10^{7}, 1$ (NMPC), nr. Pazar, c. $24 \mathrm{~km}$ $\mathrm{W}$ of Tokat $\left(40^{\circ} 16^{\prime} \mathrm{N}, 36^{\circ} 17^{\prime} \mathrm{E}\right)$, Ballica, nr. Mağarasi cave, $29.06-$ 1.07.2002, MR; 1 + (NHMW), Kizilcahaman, 1000-1200 m a.s.l., thicket of fruit trees, 27-28.05.1967, JG, FR \& AR; $10^{7}$ (MHNG), Kars, c. $16 \mathrm{~km} \mathrm{SW}$ of GOle, sieving of leaf- and moss-litter in oak and hornbeam forest, c. $1600 \mathrm{~m}$ a.s.1., 16.06.1986, C. Besuchet, I. Löbl \& D.H. Burckhardt.

COMMENTS. A Euro-Siberian subboreal species, known from central Europe [Helsdingen, 2014] to NW China (Xinjiang) [Logunov, Marusik, 2000b]. New record for Turkey.

Synageles albotrimaculatus (Lucas, 1846)

MATERIAL. SPAIN: 1 o7 (MMUM; G7512.1992), Sierra de Cazorla, in grassland on rocky hills at $1800 \mathrm{~m}$ a.s.1., 23.05.1968, ED

COMMENTS. A Mediterranean subboreal species, known from Spain and Algeria to Turkey [JiménezValverde, Wesołowska, 2005; Coşar et al., 2014].

Synageles dalmaticus (Keyserling, 1863)

MATERIAL. GREECE: 2 우 (MHNG), Cyclades, Iraklia (island $\mathrm{S}$ of Naxos), Aghio Georgios, rocks on the shore covere 'phrygana' vegetation, $15-20 \mathrm{~m}$ a.s.1., 10.05.1985, CL; 1 o' (MHNG), Naxos, foothill of Mt. Zeus, 420-490 m a.s.1., 14.05.1985, CL; 1 + (SMFM), S coast of Peloponnes, Ligia, beach, dunes and adjacent cultural landscape, 1.06.1998, LM. - TURKEY: 2 우 (LMNM), Silifke, Mersin, sand dune system with scattered scrub up to $400 \mathrm{~m}$ from sea, 10.06.1993, SJ \& CF; 1 + (LMNM), Içel,

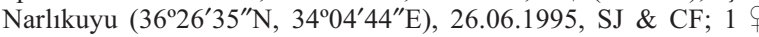
(LMNM), Adana $\left(36^{\circ} 33^{\prime} \mathrm{N}, 35^{\circ} 19^{\prime} \mathrm{E}\right)$, Burnu, Karatas, mixed vegetation between sea channel and sand dunes, 28.06.1995, SJ \& CF $1 \%$ (LMNM), Içel, c. $2 \mathrm{~km} \mathrm{~S}$ of Çamlidere $\left(36^{\circ} 52^{\prime} 12^{\prime \prime} \mathrm{N}, 34^{\circ}\right.$ $24^{\prime} 37^{\prime \prime} \mathrm{E}$ ), rocky disuded quarry at side of road, dominated by Euphorbia, Mullein Cistus and Rubus, 30.06.1995, SJ \& CF; 1 O (NHMW), Namrun, pine forest, 12.05-5.06.1964, FR; 1 o (NHMW), Demre, 30.04.1964, FR; $1 \mathrm{O}^{\text {T}}$ (NHMW), Kaş, 23.04.1964, FR; $10^{7}, 1$ ( 1 (NUAM), Antalya Prov., Alanya Distr., Cebireis Mt. c. $900 \mathrm{~m}$ a.s.1., 16.05.2006, D. Hakan. - EGYPT: $1 \sigma^{7}$ (SMFM, 4776), Cairo, no date and collector name. - ISRAEL: 1 ( (NMPC), Sede Boqer, Negev desert, between Beer Sheva and Mitzpe Ramon, 6-29.05.2003, MR.

COMMENTS. A Mediterranean subboreal species, known from the Iberian Peninsula [Helsdingen, 2014], throughout Asia Minor and the Near East [Karol, 1967; Prószyński, 2003; present data] to Azerbaijan [Logunov, Guseinov, 2002].

Synageles hilarulus (C.L. Koch, 1846)

MATERIAL. SPAIN: $1 \sigma^{7}$ (MVHN), Valencia, Devesa del Saler (Polideportivo), 17.05.2004, DD. - CYPRUS: 1 (PCSD),
Akrotiri, Fassouri reedbeds, rank grasses, 4.05.2007, R.G. Snazell; 2 우 (PCRS), Akrotiri, edge of Fassouri reedbeds, swept herbs \& grass, 4.05.2007, coll.?.

COMMENTS. A trans-Eurasian subboreal species [Logunov, Marusik, 2000b]. New record for Cyprus.

Synageles persianus Logunov, 2004

MATERIAL. ARMENIA: 2 +ᄋ (ZMUM), nr. Megri, 3.06.1988, V.A. Zakharyan.

COMMENTS. Recorded from the Caucasus (Armenia and Azerbaijan) and NW Iran only [Logunov, 2004a; Marusik et al., 2004; present data]. New record for Armenia.

Talavera aequipes (O. Pickard-Cambridge, 1871)

MATERIAL. ISRAEL: $10^{\top}$ (ZTAU), Upper Galilee, Mt. Meron, Ziv'on (c. 33 $3^{\circ} 1^{\prime} 5.84^{\prime \prime} \mathrm{N}, 35^{\circ} 24^{\prime} 32.04^{\prime \prime} \mathrm{E}$ ), 4-11.04.2007, T. Levanony; 1 क (ZTAU), Upper Galilee, Mt. Meron (c. $33^{\circ} 00^{\prime} \mathrm{N}, 35^{\circ}$ $\left.24^{\prime} \mathrm{E}\right), 800 \mathrm{~m}$ a.s.1., $12-19.05 .2007$, T. Levanony. - AZERBAIJAN: 1 을 (SZMN), Lenkoran Area, Lenkoran Reserve (c. $38^{\circ} 38^{\prime} \mathrm{N}$, $\left.48^{\circ} 47^{\prime} \mathrm{E}\right), 23.05 .2003$, EG.

COMMENTS. A Euro-Siberian - Cenral Asian temperate species, known from Spain, throughout Europe and the Mediterranean to Transbaikalia [Helsdingen, 2014; Logunov, Marusik, 2000b], southward to Israel [present data].

\section{Talavera aperta (Miller, 1971)}

MATERIAL. TURKEY: 1 (LMNM), Kayseri, Sultansazligi, degraded edge of marsh with Atriplex, Suaeda, Scirpus, Carex and various grasses, 14.06.1993, SJ \& CF.

COMMENTS. A Euro-Siberian subboreal species, known from Belgium to Transbaikalia and S Kazakhstan [Logunov, Kronestedt, 2003]. New record for Turkey.

\section{Talavera milleri (Brignoli, 1983)} Fig. 106.

MATERIAL. PORTUGAL: $1 \sigma^{\top}$ (FMNH), Planalto de S. Mamede, pitfall traps, 4.06.2002, PC. - CZECH REPUBLIC: $1 \sigma^{7}$ (NMPC), North Bohemia, Louny, Oblík Hill, rocky steppe $480 \mathrm{~m}$ altitude, 29.05.1962 J. Buchar.

COMMENTS. Recorded from Portugal, Czech Republic, Germany and Slovakia only [Chvátalová, Buchar, 2002; WSC, 2015; present data]. New record for Portugal.

Talavera petrensis (C.L. Koch, 1837)

MATERIAL. PORTUGAL: $10^{7}, 1$ (FMNH), Vale Garcia (c. $\left.39^{\circ} 33^{\prime} \mathrm{N}, 8^{\circ} 35^{\prime} \mathrm{W}\right), 2-21.07 .2002$, PC.

COMMENTS. A European - Central Asian species, recorted from Ireland and Portugal to E Kazakhstan, southward to Kyrgyzstan [Logunov, Kronestedt, 2003; present data].

\section{Thyene imperialis (Rossi, 1846)}

MATERIAL. ALGERIA: $10^{7}$ (PCRB), Wilaya Biskra, Biskra, c. $130 \mathrm{~m}$ a.s.l., flooded park, between Graminea, 10.04.1982, RB; $1 \sigma^{7}$ (PCRB), Wilaya Biskra, Biskra, nr. hotel Hamman Saline, c. $125 \mathrm{~m}$ a.s.l., under stones, 3.11.1987, RB. - TUNISIA: $3 \sigma^{7} \sigma^{7}, 1$ O (PCRB), Gouvernorat Beja, Wadi Zarga, c. $300 \mathrm{~m}$ a.s.1., in Olea orchard (on trees), 12.09.1985, V. Bouters; $1 \mathrm{O}^{\top}$ (OUMNH), Sfax Distr., Qued Mealn el Jem ( $\left.35^{\circ} 09^{\prime} 02^{\prime \prime} \mathrm{N}, 10^{\circ} 43^{\prime} 50^{\prime \prime} \mathrm{E}\right), 34 \mathrm{~m}$ a.s.1., beaten from Tamarix, 30.04.2005, D. Mann. - ITALY: 1 (SMFM, 11/986), Sicily, no exact locality and collector name. - GREECE: 
$1 \sigma^{7}$ (SMFM), S Crete, Keratokampos, 7.05.2008, HE \& UK; 1 ○ (SMFM), S Crete, Plakias, 21.07.2009, HE \& UK; $1 \sigma^{7}$ (SMFM), NE Greece, Kavousi, 10.05.2009, KE \& UK; 1 \% (SMFM), N Crete, Gournes, 3.05.2011, S. Krips. - TURKEY: 1 ㅇ (PCRS), road between Akyaka and Gogovia, in weeds, etc. edge of road, 4.06.1996, ARS; 1 (LMNM), Silifke, Mersin, sand dune system with scattered scrub up to $400 \mathrm{~m}$ from sea, 10.06.1993, SJ \& CF; 4 $\sigma^{\top} \sigma^{7}, 2$ 우 (LMNM), Içel, Silifke Sand Dunes $\left(36^{\circ} 17^{\prime} 05^{\prime \prime} \mathrm{N}, 33^{\circ}\right.$ $\left.56^{\prime} 08^{\prime \prime} \mathrm{E}\right)$, inner dunes with damp, Salicornia dominated, halophytic vegetation and red bed, 5.05.1994, SJ \& CF; $1 \sigma^{7}$ (LMNM), Içel, Narlikuyu $\left(36^{\circ} 26^{\prime} 04^{\prime \prime} \mathrm{N}, 34^{\circ} 06^{\prime} 44^{\prime \prime} \mathrm{E}\right)$, garrigue vegetated limestone dominated by Pinus, 5.06.1994, MB; 1 ऊ (LMNM), Içel, c. 17 km $\mathrm{N}$ of Anamur, Alaköprü Bridge $\left(36^{\circ} 10^{\prime} 38^{\prime \prime} \mathrm{N}, 32^{\circ} 53^{\prime} 51^{\prime \prime} \mathrm{E}\right)$, wet flush on road, downward slope to river bank and rocky outcrop, 7.05.1994, SJ \& CF; 1 q (LMNM), Içel, c. $2 \mathrm{~km} \mathrm{~S}$ of Çamlidere $\left(36^{\circ} 52^{\prime} 12^{\prime \prime} \mathrm{N}, 34^{\circ} 24^{\prime} 37^{\prime \prime} \mathrm{E}\right)$, rocky disuded quarry at side of road, dominated by Euphorbia, Mullein Cistus and Rubus, 30.06.1995, SJ \& CF; 1 (SMFM), Killis, Ortsbereich $\left(36^{\circ} 43^{\prime} \mathrm{N}, 37^{\circ} 07^{\prime} \mathrm{E}\right), 7-$ 8.08.1988, coll. Zoologie THD; 1 ㅇ (NHMW), Finibe, 1.05.1964, FR. - EGYPT: 1 o (NHMW), El-Fayem [apparently Faiyum, $29^{\circ} 27^{\prime} \mathrm{N}, 30^{\circ} 35^{\prime} \mathrm{E}$ ], 12.1981, HEH. - ISRAEL: 1 \% (SMFM) Rehoboth-Jaffa [Rehovot], 18.04.1913, J. Aharoni. - SAUDI ARABIA: $1 \sigma^{7}$ (SMFM), c. $150 \mathrm{~km}$ SSE of El Riyadh, 13.06.1959, E. Diehl.

COMMENTS. A trans-Eurasian subboreal-subtropical species, known from the Canaries [Helsdingen, 2014], throughout the Mediterranean and northern Africa, to China and India [Logunov, Zamanpoore, 2005]. In Africa, this species was hitherto recorded from Sudan (Jebel Marra) [Logunov, 2004b], Mali (Bamako), Guinea (Kindia), Côte d'Ivoire (as Batié, apparently it is now Beuna) and Burkina (Ouagadougou) [Berland, Millot, 1941]. All the African localities by Berland \& Millot [1941] represent the south-westernmost records for T. imperialis.

Yllenus albocinctus (Kroneberg, 1875)

MATERIAL. RUSSIA: 1 (ZMUM), Astrakhan’ Region, Lishansky Distr., E of Basinskaya station $\left(46^{\circ} 07^{\prime} 55^{\prime \prime} \mathrm{N}, 47^{\circ} 13^{\prime} 05^{\prime \prime} \mathrm{E}\right)$ 18.05.2012, AG.

COMMENTS. An eastern Mediterranean - Central Asian subboreal species, known from Turkey to $\mathrm{W}$ Mongolia [Logunov, Marusik, 2003].

Yllenus gavdos Logunov et Marusik, 2003

MATERIAL. ITALY: $10^{7}$ (PCMS, Sa31), Sardinia, 20.06.2013, M. Schäfer.

COMMENTS. Recorded the Canaries, Italy, Greece (Crete) and Algeria only [Logunov, Marusik, 2003; present data]. New record for Italy (Sardinia).

Yllenus saliens O. Pickard-Cambridge, 1876

MATERIAL. EGYPT: $20^{7} \sigma^{7}, 3$ 우 (SMFM, 5523), 'Pharo' (label illegible), 2.10.1933, coll.?

COMMENTS. Recorded from NE Africa, including the Arabian Peninsula, only [Logunov, Marusik, 2003; Wesołowska, van Harten, 2007; present data].

Yllenus univittatus (Simon, 1871)

MATERIAL. TURKEY: 1 ๆ (LMNM), Konya, Sakyatan, 10.07.1992, SJ.

COMMENTS. Recorded from France and Turkey only [Logunov, Marusik, 2003; present data]. New record for Turkey.

\section{Discussion}

The Mediterranean fauna of Salticidae remains studied insufficiently, considering both the regional state of knowledge (i.e., regional faunistic lists) and distribution of particular species over the Mediterranean and beyond. At present, the best studied regions are the Iberian Peninsula (124 species; both Portugal and Spain) [Cardoso, Morano, 2010], Italy (135 species) [Pantini, Isaia, 2014], Greece (c. 140 species; both mainland and such large islands as Crete, Lesbos and Chios) [Bosmans, Chatzaki, 2005; Bosmans et al., 2009, 2013; Russell-Smith et al., 2011], Bulgaria (91 species) [Deltshev, 2005], Egypt (72 species) [El-Hennawy, 2006], Romania (83 species) [Weiss, István, 2009], Turkey (110 species) [Bayram et al., 2014; Coşar, 2014], Israel (108 species) [Zonstein, Marusik, 2013], and the Crimea (68 species) [Kovblyuk, 2004; Kovblyuk, pers. comm.]. The least studied Mediterranean regions are those of northern Africa (Algeria, Tunisia, Libya). For instance, the salticid fauna of Tunisia currently consists of 37 species only [Bosmans, 2003], which is hardly more than $15 \%$ of its real regional diversity. Yet, some of the regional lists, e.g. that of the Lebanese fauna [El-Hennawy, 2013], are largely based on old records originating from 19 th century and thus are in need of revision.

The most problematic Mediterranean genera of Salticidae requiring thorough taxonomic revisions are as follows: Euophrys C.L. Koch, 1834 (s.str.), Habrocestum Simon, 1876, Pellenes Simon, 1876 and Salticus Latreille, 1804. Some obscure earlier records of Philaeus - e.g., P. albovariegatus (Simon, 1868), P. jugatus (L. Koch, 1876) and others (listed in Cardoso \& Morano [2010]) — need a critical revision and/or modern confirmation.

With the current state of the knowledge of the Mediterranean Salticidae, it is hardly surprising that the present faunistic survey has resulted in numerous additional records to regional faunistic lists (in alphabetic order): Armenia (2 new species records), Cyprus (19), Egypt (4), Greece (1), Israel (1), Italy (1), Jordan (2), Libya (1), Morocco (4), Oman (1), Portugal (3), Spain (3), Syria (8), Tunisia (1), Turkey (17), Ukraine (1). The ranges of some unrecorded species turned out to be much larger than it was originally known: for instance, Talavera milleri has been found in Portugal (earlier known from Germany, Czech Repulic and Slovakia only [Chvátalová, Buchar, 2002]), and Yllenus univittatus has been recorded from Turkey (earlier known from France only [Logunov, Marusik, 2003]). Unknown sexes have been described for seven species, of which some have been known from the single sex from the 19th century: Habrocestum latifasciatum ( $(+)$, H. shulovi $\left(\mathrm{O}^{7}\right)$, Heliophanus fuerteventurae (†), H. ramosus (+), Icius crassipes (+), I. insolitus $(+)$ and Salticus confusus (+). Currently, it is impossible to provide a correct estimate of real diversity of the Mediterranean Salticidae; in my opinion, there should be at least 300350 species, but it is likely to be more than that. 
ACKNOWLEDGEMENTS. The author wishes to express his warmest thanks to all the curators and private collectors mentioned above under 'Material and Methods' for giving access to their collections of Salticidae. G.N. Azarkina (the SZMN) is thanked for the help in identification of difficult Aelurillus species and providing me with additional records of Salticidae from Azerbaijan. Anna Logunova (Manchester, UK) is thanked for the translation of locality labels from German and French. Yu.M. Marusik (Magadan, Russia) is obliged for his critical comments on the earlier draft helping to improve it.

\section{References}

Alicata P., Cantarella T.1994. The Euro-mediterranean species of Icius (Araneae, Salticidae): a critical revision and description of two new species // Animalia. Vol.20 (for 1993). No.1/3. P.111-131.

Azarkina G.N. 2002. New and poorly known species of the genus Aelurillus Simon, 1884 from central Asia, Asia Minor and the eastern Mediterranean (Araneae: Salticidae) // Bull. Br. arachnol. Soc. Vol.12. Pt.6. P.249-263.

Azarkina G.N. 2004 (for 2003). New and poorly known species of the genus Phlegra Simon, 1876 (Araneae, Salticidae) // Revue Arachnologique. T.14. Fasc.6. P.73-108.

Azarkina G.N., Logunov D.V. 2006. Taxonomic notes on nine Aelurillus species of the western Mediterranean (Araneae: Salticidae) // Bull. Br. arachnol. Soc. Vol.13. Pt.7. P.233-248.

Azarkina G.N., Mirshamsi O. 2014. Description of a new Aelurillus species from Khorasan province of Iran, with comments on A. concolor Kulczyński, 1901 (Araneae: Salticidae) // Zoology in the Middle East. Vol.60. No.1. P.82-91. doi:10.1080 09397140.2014 .892353

Bayram A., Kunt K.B., Danısman T. 2015. The checklist of the spiders of Turkey (Araneae; Arachnida), version 2014; online at: http://www.kazimcapaci.com/spidersofturkey.htm (accessed on January 22, 2015)

Berland L., Millot J. 1941. Les araignées de l'Afrique Occidentale Française I.-Les salticides // Mém. Mus. natn. Hist. nat. Paris (N.S.). Vol.12. P.297-423.

Bosmans R. 2003. A checklist of the spiders of Tunisia, with description of a new species of Palliduphantes Saaristo \& Tanasevitch (Araneae: Linyphiidae). // Kaupia - Darmstädter Beiträge zur Naturgeschichte. Bd.12. S.89-109.

Bosmans R., Chatzaki M. 2005. A catalogue of the spiders of Greece, a critical review of all spider species cited from Greece with their localities // Newsletters Belg. Arachnol. Soc. Vol.20. No.2(suppl.). P.1-124.

Bosmans, R., Baert L., Bosselaers J., De Koninck H., Maelfait J.P., van Keer J. 2009. Spiders of Lesbos (Greece) // Nieuwsbrief van de Belgische Arachnologische Vereniging Vol.24. P.1-70.

Bosmans R., Van Keer J., Russell-Smith A., Kronestedt T., Alderweireldt M., Bosselaers J., De Koninck H. 2013. Spiders of Crete (Araneae). A catalogue of all currently known species from the Greek island of Crete // Newsletter Belg. Arachnol. Soc. Supplement 1. Vol.28. P.1-147.

Canard A. 2005. Catalogue of spider species from Europe and the Mediterranean basin, parts I \& II // Revue Arachnologique. Vol.15. Fasc.3. P.1-255.

Chvátalová I., Buchar J. 2002. Distribution and habitat of Talavera aperta, T. milleri and T. thorelli in the Czech Republic (Araneae: Salticidae) // Acta Societatis Zoologicae Bohemicae. Vol.66. P.3-11.

Coşar İ. 2014. Four new records for the spider fauna of Turkey (Araneae: Salticidae) // Turkish J. Zool. Vol.38. P.1-4.

Coşar İ., Danişman T., Yildiran F.A.B. 2014. The jumping spiders' fauna of Kirikkale Province (Araneae, Salticidae) // Serket. Vol.14. No.2. P.83-94.

Danişman T., Coşar İ., Erdek M., Sansak Z. 2012. A contribution to the knowledge of Turkish jumping spider fauna (Araneae: Salticidae) // Acta Zool. Bulg. Vol.64. No.2. P.215-216.
Deltshev C. 2005. Fauna and zoogeography of spiders (Araneae) in Bulgaria // J. Arachnol. Vol.33. P.306-312.

Dobroruka L.J. 2002. Notes on a collection of jumping spiders from Greece, mainly from Crete (Araneae: Salticidae) // Biologia Gallo-hellenica. Vol.28. P.5-26.

Dondale C.D., Redner J.H., Marusik Yu.M. 1997. Spiders (Araneae) of the Yukon // Danks H.V., Downes J.A. (eds.). Insects of the Yukon. Ottawa: Biological Survey of Canada (Terrestrial Arthropods). P.73-113.

Dunin P.M. 1984. [Fauna and ecology of the spiders of the Apsheron Peninsula, Azerbadjan SSR] // Utotchkin A.S. (ed.). Fauna i ekologiya paukoobraznykh. Perm: University of Perm. P.4560 [in Russian].

El-Hennawy H.K. 2006. A list of Egyptian spiders (revised in 2006) // Serket. Vol.10. No.2. P.65-76.

El-Hennawy H.K. 2013. Preliminary list of Lebanese spiders and other arachnids (except ticks and mites) // Serket. Vol.13. No.3/4. P.228-275.

Fiser C., Azarkina G.N. 2005. A contribution to the knowledge of the jumping spiders (Salticidae: Araneae) of the Macedonia // Acta Zool. Bulg. Vol.57. No.3. P.299-304.

Heciak S., Prószyński J. 1984. Redescriptions of one Aelurillus and two Phlegra species (Araneae, Salticidae) from Spain // Ann. zool. Warszawa. T.37. No.14. P.377-390.

Helsdingen van P. 2013. A quick scan of the spider fauna of the European part of Turkey // Nieuwsbrief SPINED. No.33. P.2938.

Helsdingen van P. 2014. Fauna Europaea: Araneae. Fauna Europaea version 2.6.2; online at: http://www.faunaeur.org/ (accessed on December 22, 2014)

Jäger P. 1995. Erstnachweise von Macaroeris nidicolens und Icius subinermis für Deutschland in Köln (Araneae: Salticidae) // Arachnologische Mitteilungen. H.9. S.38-39.

Jiménez-Valverde A., Wesołowska W. 2005. Synageles albotrimaculatus (Lucas, 1846) (Araneae, Salticidae), a spider species new to Spain // Bull. Br. Arachnol. Soc. Vol.13. P.122124.

Ijlnad S., van Helsdingen P. 2014. On some spiders (Arachnida, Araneae) from the surroundings of Castellabate, Italy // NieuwsbriefSPINED. No.34. P.16-33.

Karol S. 1967. Türkiye örümcekleri I. Ön liste // Ankara Üniv. Fak. Yayinlari. Vol.109. No.7. P.1-34. [in Turkish]

Koch C.L. 1846. Die Arachniden. Nürnberg. Dreizehnter Band. S.1-234; Vierzehnter Band. S.1-88.

Kovblyuk M.M. 2004. [Catalogue of the spiders (Arachnida, Aranei) of the Crimea] // Voprosy razvitiya Kryma. Nauchnoprakticheskiy i diskussionno-analiticheskiy sbornik. Vyp.15. Problemy inventarizatsii krymskoi bioty. Simferopol: TavriyaPlus. P.211-262 [in Russian, with English and Ukrainian summaries].

Kulczyński W. 1903. Arachnoidea in Asia Minore et ad Constantinopolim a Dre F. Werner collecta // Sitz.-ber. Akad. Wiss. Wien. Bd.112. S.627-680.

Logunov D.V. 1992. Salticidae of Middle Asia (Araneae). I. New species from the genera Heliophanus, Salticus and Sitticus, with notes on new faunistic records of the family // Arthropoda Selecta. Vol.1. No.1. P.51-67.

Logunov D.V. 1995. New and little known species of the jumping spiders from Central Asia (Araneae: Salticidae) // Zoosystematica Rossica. Vol.3. No.2. P.237-246.

Logunov D.V. 1996. A review of the genus Phlegra Simon, 1876 in the fauna of Russia and adjacent countries (Araneae: Salticidae: Aelurillinae) // Genus. Vol.7. No.3. P.533-567.

Logunov D.V. 1997. Salticidae of Middle Asia. 4. A review of the genus Euophrys (s. str.) C.L. Koch (Araneae, Salticidae) // Bull. Br. arachnol. Soc. Vol.10. Pt.9. P.344-352.

Logunov D.V. 1998a. Two new jumping spider species from the Caucasus (Aranei: Salticidae) // Arthropoda Selecta. Vol.7. No.4. P.301-303.

Logunov D.V. 1998b. New species and new records of Palaearctic Sitticus (Araneae: Salticidae) // Zoosystematica Rossica. Vol.7. No.1. P.77-83. 
Logunov D.V. 1999. Redefinition of the genus Habrocestoides Prószyński, 1992, with establishment of a new genus, Chinattus gen.n. (Araneae: Salticidae) // Bull. Br. arachnol. Soc. Vol.11. Pt.4. P.139-149.

Logunov D.V. 2001a (for 2000). A redefinition of the genera Bianor Peckham \& Peckham, 1885 and Harmochirus Simon, 1885 , with the establishment of a new genus Sibianor gen. n. (Araneae: Salticidae) // Arthropoda Selecta. Vol.9. No.4. P.221-286.

Logunov D.V. 2001b. New and poorly known species of the jumping spiders (Araneae: Salticidae) from Afghanistan, Iran and Crete // Arthropoda Selecta. Vol.10. No.1. P.59-66.

Logunov D.V. 2001c. Literature review: Die Springspinnen (Araneae, Salticidae) Griechenlands (by H. Metzner) // Newsl. Br. arachnol. Soc. No.92. P.6-7.

Logunov D.V. 2004a. Notes on new and poorly known Palaearctic species of the genera Neon, Sitticus and Synageles (Araneae: Salticidae) // Bull. Br. arachnol. Soc. Vol.13. Pt.2. P.33-40.

Logunov D.V. 2004b. Taxonomic notes on a collection of jumping spiders from Sudan (Araneae, Salticidae) // Bull. Br. arachnol. Soc. Vol.13. Pt.3. P.86-90.

Logunov D.V. 2009a. Further notes on the Harmochireae of Africa (Araneae, Salticidae, Pelleninae) // Stoev P., Dunlop J., Lazarov S. (eds.). A life caught in a spider's web. Papers in arachnology in honour of Christo Deltshev. ZooKeys. Vol.16. P.265-290.

Logunov D.V. 2009b. New and poorly known species of Salticidae (Araneae) from Turkey and Iran // Kropf C., Horak P. (eds) Towards a natural history of arthropods and other organisms. In memoriam Konrad Thaler. Contributions to Natural History. Vol.12. P.899-919.

Logunov D.V. 2010. Taxonomic notes on a collection of jumping spiders from Iran (Araneae, Salticidae) // Bull. Br. arachnol. Soc. Vol.15. Pt.3. P.85-90.

Logunov D.V. 2012. Notes on a small spider collection from Turkey (Arachnida: Aranei) // Arthropoda Selecta. Vol.21. No.4 P.375-377.

Logunov D.V. 2013. On a small spider collection from Cyprus (Arachnida: Araneae) // Newsl. Br. Arachnol. Soc. No.127. P.4-5.

Logunov D.V., Azarkina G.N. 2006. New species and records of Phlegra from Africa (Araneae, Salticidae) // Revue suisse de Zoologie. T.113. Fasc.4. P.727-746.

Logunov D.V., Chatzaki M. 2003. An annotated check-list of the Salticidae (Araneae) of Crete, Greece // Revista Ibérica de Arachnologia. Vol.7. P.95-100.

Logunov D.V., Guseinov E.F. 2002. Faunistic review of the jumping spiders of Azerbaijan (Aranei: Salticidae), with additiona faunistic records from neighbouring Caucasian countries // Arthropoda Selecta. Vol.10 (for 2001). No.3. P.243-260.

Logunov D.V., Kronestedt T. 2003. A review of the genus Talavera Peckham \& Peckham, 1909 (Araneae, Salticidae) // Journal of Natural History. Vol.37. No.9. P.1091-1154.

Logunov D.V., Marusik Yu.M. 1999. A brief review of the genus Chalcoscirtus Bertkau, 1880 in the faunas of Central Asia and the Caucasus (Aranei: Salticidae) // Arthropoda Selecta. Vol.7 (for 1998). No.3. P.205-226.

Logunov D.V., Marusik Yu.M. 2000a. Miscellaneous notes on Palaearctic Salticidae (Arachnida: Aranei). Arthropoda Selecta. Vol.8 (for 1999). No.4. P.263-292.

Logunov D.V., Marusik Yu.M. 2000b. Catalogue of the jumping spiders of northern Asia (Arachnida, Araneae, Salticidae). Moscow: KMK Scientific Press. 299 pp.

Logunov D.V., Marusik Yu.M. 2003. A revision of the genus Yllenus Simon, 1868 (Arachnida, Araneae, Salticidae). Moscow: KMK Scientific Press. 167 pp.

Logunov D.V., Marusik Yu.M., Mozaffarian F. 2002. Faunistic review of the jumping spiders of Iran (Aranei: Salticidae) // Arthropoda Selecta. Vol.10 (for 2001). No.2. P.155-167.

Logunov D.V., Marusik Yu.M., Rakov S.Yu. 1999. A review of the genus Pellenes in the fauna of Central Asia and the Caucasus // Journal of Natural History. Vol.33. P.89-148.

Logunov D.V., Mirshamsi O., Musavi S., Shayestehfar A. 2013. New faunistic records of Salticidae (Aranei) from Khorasan Province of Iran // Arthropoda Selecta. Vol.22. No.4. P.379-381.
Logunov D.V., Rakov S.Yu. 1998. Miscellaneous notes on Middle Asian jumping spiders (Aranei: Salticidae) // Arthropoda Selecta. Vol.7. No.2. P.117-144.

Logunov D.V., Vazirianzadeh B., Moravvej S.A., Navidpour S. 2007. New faunistic records of the jumping and crab spiders (Araneae: Salticidae, Thomisidae and Philodromidae) from Iran // Arthropoda Selecta. Vol.15 (for 2006). No.3. P.225-228

Logunov D.V., Zamanpoore M. 2005. Salticidae (Araneae) of Afghanistan: an annotated check-list, with descriptions of four new species and three new synonyms // Bull. Br. arachnol. Soc. Vol.13. Pt.6. P.217-232.

Lucas H. 1846. Histoire naturelle des animaux articules // Exploration scientifique de l'Algerie pendant les annees 1840, 1841, 1842 publiee par ordre du Gouvernement et avec le concours d'une commission academique. Paris, Sciences physiques, Zoologie. Vol.1. P.89-271.

Marusik Yu.M., Guseinov E.F., Aliev H.A. 2004. Spiders (Arachnida: Aranei) of Azerbaijan 4. Fauna of Naxçivan // Arthropoda Selecta. Vol.13. No.3. P.135-149.

Mello-Leitão C.F. de. 1944. Arañas de la provincia de Buenos Aires // Revta Mus. La Plata (N.S., Zool.). Vol.3. P.311-393.

Metzner H. 1999. Die Springspinnen (Araneae, Salticidae) Griechenlands // Andrias. Bd.14. S.1-279.

Nentwig W., Blick T., Gloor D., Hänggi A., Kropf C. 2014. Spiders of Europe (version 12.2014), online at: www.araneae.unibe.ch (accessed on December 21, 2014).

Nosek A. 1905. Araneiden, Opilionen und Chernetiden // Penther A., Zederbauer E., Ergebnisse einer naturwissenschaftlichen Reise zum Erdschias-Dagh (Kleinasien). Annalen des Kaiserlich-Königlichen Naturhistorischen Hofmuseums in Wien. Bd.20. S.114-154

Ono H. 1988. A revisional study of the spider family Thomisidae (Arachnida, Araneae) of Japan. Tokyo: National Science Museum. $252 \mathrm{pp}$.

Özșen Ö., Türkes T. 2009. The first record of genus Phintella (Araneae: Salticidae) in the Turkish spider fauna // Serket. Vol.11. No.3/4. P.79-81.

Pantini P., Isaia M. 2014. La checklist dei ragni italiani (version December 2014); online at: http://www. museoscienzebergamo.it/web/index.php?option $=$ com content \&view $=$ category\&layout $=$ blog\&id $=96 \&$ Itemid $=94$ (assessed on January 25, 2015).

Prószyński J. 2000. On mostly new species of Salticidae (Aranei) from Levant // Arthropoda Selecta. Vol.8 (for 1999). No.4. P.231-262.

Prószyński J. 2003. Salticidae (Araneae) of the Levant// Ann. zool. Warszawa. T.53. P.1-180.

Prószyński J. 2014. Monograph of the Salticidae (Araneae) of the World 1995-2012. Global Species Database of Salticidae (Araneae) (version December 31, 2014), online at: http://www. peckhamia.com/salticidae/index.html (accessed on January 22, 2015).

Rakov S.Yu., Logunov D.V. 1996. A critical review of the genus Heliophanus C.L. Koch, 1933, of Middle Asia and the Caucasus (Aranei: Salticidae) // Arthropoda Selecta. Vol.5. No.3/4. P.67-104.

Rakov S.Yu., Logunov D.V. 1997. Taxonomic notes on the genus Menemerus Simon, 1868 in the fauna of Middle Asia (Araneae, Salticidae) // Żabka M. (ed.). Proc. 16th Europ. Coll. Arachnol. P.271-279.

Russell-Smith A., Allison R., Askins M., Blumsom W., Snazell R., Spilling C. 2011. A provisional checklist and gazetteer of the spiders of Chios, Greece (Arachnida: Araneae) // Bull. Br. Arachnol. Soc. Vol.15. Pt.5. P.133-167.

Schmidt G., Krause R.H. 1996. Weitere Spinnenfunde von den Kanarischen Inseln, hauptsächlich von Fuerteventura und Lobos (Arachnida: Araneae) // Faunist. Abhandl. staatl. Mus. Tierk. Dresden. Bd.20. S.259-273.

Simon E. 1868. Monographie des espèces européennes de la famille des attides (Attidae Sundewall. - Saltigradae Latreille) // Annales de la Société Entomologique de France. Ser.4. T.8. P.11$72,529-726$ 
Song D.X., Zhu M.S., Chen J. 1999. The Spiders of China. Shijiazhuang: Hebei Sci. Technol. Publ. House. 640 pp.

Topçu A., Demir H., Seyyar O., Türkeş T. 2006. The spider fauna of the Gülek Pass (Turkey) and its environs (Araneae) // Deltshev C., Stoev P. (eds.). European Arachnology 2005, Acta Zool. Bulg. Suppl. No.1. P.287-295.

Topçu A., Demir H., Seyyar O. 2009. Contribution to the jumping spider fauna of Turkey (Araneae: Salticidae) // Zoology in the Middle East. Vol.47. P.112-113.

Trotta A. 2005. Introduzione ai Ragni italiani (Arachnida Araneae) // Mem. Soc. Entomol. ital. Vol.83. P.3-178.

Uyar Z., Uğurtaș İ.H. 2012. Jumping Spiders (Araneae: Salticidae) of Northwest Anatolia (Turkey) // Acta Zool. Bulg. Vol.64. No.3. P. $235-240$.

Weiss I., István U. 2009. Faunenliste der Spinnen Rumäniens (Arachnida: Araneae); online at: http://www.arachnologie.info/ fauna.htm (accessed on January 22, 2015).

Wesołowska W. 1986. A revision of the genus Heliophanus C.L. Koch, 1833 (Aranei: Salticidae) // Ann. zool. Warszawa. T.40. P.1-254.

Wesołowska W. 1996. New data on the jumping spiders of Turkmenistan (Aranei Salticidae) // Arthropoda Selecta. Vol.5. No.1/ 2. P.17-53.

Wesołowska W. 1999. A revision of the spider genus Menemerus in Africa (Araneae: Salticidae) // Genus. Vol.10. No.2. P. 251-353.

Wesołowska W., van Harten A. 1994. The jumping spiders (Salticidae, Araneae) of Yemen. Sana'a: Yemeni-German Plant Protection Project. $86 \mathrm{pp}$.

Wesołowska W., van Harten A. 2007. Additions to the knowledge of jumping spiders (Araneae: Salticidae) of Yemen // Fauna of Saudi Arabia. Vol.23. P.189-269.
Wesołowska W., van Harten A. 2010. Order Araneae, family Salticidae // van Harten A. (ed.). Arthropod Fauna of the UAE. Dar Al Ummah, Abu Dhabi. Vol.3. P.27-69.

Wesołowska W., van Harten A. 2011. Order Araneae, family Salticidae: Additions and the descriptions of a new species // van Harten A. (ed.). Arthropod Fauna of the UAE. Dar Al Ummah, Abu Dhabi. Vol.4. P.23-28.

Wesołowska W., Szeremeta M. 2001. A revision of the ant-like salticid genera Enoplomischus Giltay, 1931, Kima Peckham \& Peckham, 1902 and Leptorchestes Thorell, 1870 (Araneae: Salticidae) // Insect Systematics \& Evolution. Vol.32. P.217240.

WSC. 2015. World Spider Catalog. Natural History Museum Bern, online at: http://wsc.nmbe.ch, version 15.5 (accessed on January 21,2015$)$.

Wunderlich J. 1987. Die Spinnen der Kanarischen Inseln und Madeiras: Adaptive Radiation, Biogeographie, Revisionen und Neubeschreibungen. Langen, West Germany: Triops Verlag. $435 \mathrm{~S}$.

Wunderlich J. 1992. Die Spinnen-Fauna der Makaronesischen Inseln: Taxonomie, Ökologie, Biogeographie und Evolution // Beitr. Araneol. Bd.1. S.1-619.

Wunderlich J. 2011. Extant and fossil spiders (Araneae) // Beitr. Araneol. Bd.6. S.1-640.

Yağmir E.A., Kunt K.B., Ulupınar E. 2009. A new species for the araneofauna of Turkey, Evarcha michailovi Logunov, 1992 (Araneae: Salticidae) // Munis Ent. Zool. Vol.4. No.1. P.230232.

Zonstein S.L., Marusik Yu.M. 2013. Checklist of the spiders (Araneae) of Israel // Zootaxa. Vol.3671. No.1. P.1-127.

Responsible editor K.G. Mikhailov 